\title{
Multi-Antenna System Performance and Impairments in Long Term Evolution Radio Access Networks using the Extended Spatial Channel Model
}

\author{
by \\ Michel Chauvin, B.Eng. \\ A thesis submitted to the \\ Faculty of Graduate and Postdoctoral Affairs \\ in partial fulfillment of the requirements for the degree of
}

Master of Applied Science in Electrical and Computer Engineering

Ottawa-Carleton Institute for Electrical and Computer Engineering (OCIECE)

Department of Systems and Computer Engineering

Carleton University

Ottawa, Ontario

October, 2013

(C)Copyright

Michel Chauvin, 2013 


\section{Abstract}

Consumer demand for rich content delivered to portable wireless devices is pushing the wireless industry and researchers to find ways to improve the efficiency of wireless networks. New technologies like LTE and LTE-Advanced are being refined and deployed to meet demands. This thesis studies three important areas of wireless communications using LTE; phase noise, Doppler and link adaptation and the performance of various multiple antenna systems. The thesis focuses on the performance of 4 base station antennas and results obtained with the advanced $3^{\text {rd }}$ Generation Partnership Project's (3GPP) extended spatial channel model (SCME). Simulations show the effect of each impairment and configuration on the LTE physical downlink shared channel. The best performing antenna configuration evaluated is the 4-transmitter, 4-port, correlated cross-polarized BS antenna when TM4's closed-loop spatial multiplexing is used. The results show that a 4 antenna BS setup provides gain over 2 BS antennas, despite the additional reference signal overhead, due to the greater set of precoding matrices available with 4 antenna ports. When only 2 ports are available, TM3's open-loop spatial multiplexing (OLSM) performs better than TM4 as the user equipment (UE) becomes mobile, since 2-port TM3 is less dependent on the channel state information. The practical implementation issues of link adaptation are shown to cause a significant drop in throughput at medium and high UE velocities. The results in the thesis suggest that improving the latency of the link adaptation loop with low complexity algorithms, or an increase in processing power, along with adaptive link adaptation reporting intervals can keep uplink overhead low and maintain a higher throughput as velocity increases. The UE velocity is also pushed to extremes in the high speed train on railway simulations and shows that LTE can operate with some throughput degradation at $350 \mathrm{~km} / \mathrm{hr}$. Finally, the LTE downlink is also subjected to phase noise; an important impairment present in communication systems employing up/down-conversion. The generated phase noise and the measured phase noise simulations show the effect of phase noise on the throughput. As expected, the relatively quiet measured phase noise does not significantly degrade the LTE downlink. 


\section{Acknowledgments}

I would like to thank my supervisor Prof. M. El-Tanany for his friendship and the genuine guidance he provided when I would ask for his help. He unselfishly lent me his time for any questions I had and occasional unrelated discussions about topics that interested us both. He provided encouragement along the way and allowed me to explore additional areas to satisfy my curiosity despite the extra time it would take to complete my thesis work. I'm very grateful for the opportunity he gave me to learn a new field, work on an interesting topic and meet a number of wonderful people along the way.

I would also like thank my co-workers and mentors at Ericsson who often made themselves available (at any time) to discuss projects on which we were working. They inspired me since they had a tremendous amount of passion for the work they were performing. Thank you to Edward, Ahmed, Guoqiang, Jianguo, Eliana, Xiaoming, Alireza, Jianfeng and my cubicle neighbour, Sara, for the wonderful conversations and the opportunity to learn and work with you. Thank you to Ericsson for supporting this thesis work and generously allowing me to work on topics of interest and the opportunity to work and converse with the highly skilled people in the Systems team.

There is nothing more important than family and they are the ones that inspire you and nurture curiosity from a very young age. Thank you to my parents, Cathy and Richard, who have helped me through challenging times and this most recent academic endeavor. They have shown time and time again their support, shared their wisdom and I'm forever grateful for the opportunity to explore a new field. To my wonderful and supportive family Eric, Natalie, Michael and Kaitlin, Rolande, Lise and Tony, thank you for your endless support and encouragement.

To all the friends (Hamid, Wang, Hussein, Farouk, Rudhwan, Furkan, Xiao, Matthias, Majid, Rania and many more) that I met in classes or elsewhere during our graduate 
studies, your friendship made this an unforgettable experience. I hope that you will all find work that you are passionate about as I truly believe you will all have a big impact on the world one day. 


\section{Table of Contents}

Abstract

Acknowledgments $\quad$ iv

Table of Contents $\quad$ vi

List of Tables $\quad$ x

List of Figures $\quad$ xiii

List of Symbols $\quad$ xix

List of Acronyms $\quad$ xxiii

1 Introduction 1

1.1 The Wireless Radio Access Networks . . . . . . . . . . . . . . . . . 2

1.2 LTE Radio Air Interface . . . . . . . . . . . . . . . . . . 3

1.3 Wireless Channels . . . . . . . . . . . . . . . . . 4

1.3 .1 Path Loss . . . . . . . . . . . . . . . . . . 5

1.3.2 Fading .................... 5

1.3.3 Transmit and Receive Diversity: (MISO) and (SIMO) Channels . . 6

1.3.4 Multiple-Input Multiple-Output (MIMO) Channels . . . . . . . . . 6

1.3.5 Spatial Channel Models (SCM) . . . . . . . . . . . 8

1.4 Long Term Evolution . . . . . . . . . . . . . . . . . . . 8

1.4.1 Orthogonal Frequency Division Multiplexing (OFDM) . . . . . 8

1.4.2 Link Adaptation . . . . . . . . . . . . . . 9

1.5 Thesis Motivation . . . . . . . . . . . . . . . . 10

1.6 Objectives ............................ 10 
1.7 Thesis Contributions . . . . . . . . . . . . . . . . . . 11

1.8 Organization of the Thesis $\ldots \ldots \ldots \ldots \ldots \ldots$

2 Background 14

2.1 Wireless Fading Channels . . . . . . . . . . . . . . . . . . . . . . 14

2.1.1 Path Loss and Shadowing . . . . . . . . . . . . . . . . 14

2.1.2 Multi-Path Propagation and Small-Scale Fading . . . . . . . . . 15

2.1.3 Frequency Flat and Frequency Selective Channels . . . . . . . . 19

2.2 Orthogonal Frequency Division Multiplexing (OFDM) Systems . . . . . 22

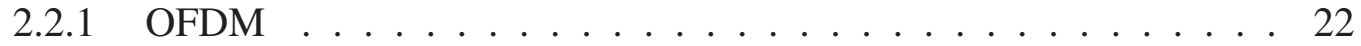

2.2.2 Cyclic Prefix $(\mathrm{CP}) \ldots \ldots \ldots \ldots$

2.3 Channel Modeling . . . . . . . . . . . . . . . . . . . 24

2.3.1 Motivation for Channel Models . . . . . . . . . . . . . . 25

2.3.2 Single Input Single Output (SISO) Wireless Channel . . . . . . . 25

2.3.3 Multi-Input Multi-Output (MIMO) Wireless Channels . . . . . . 26

2.3.4 The Received Electric Field ．. . . . . . . . . . . . . . . . 31

2.3.5 Clarke's Reference Model . . . . . . . . . . . . . . . . . . . . 34

2.3.6 Jakes' Model . . . . . . . . . . . . . . . . . . . . . . . . . . 38

2.3.7 Spatial Channel Model . . . . . . . . . . . . . . . . . . . . . . 42

2.3 .8 Simulation Model . . . . . . . . . . . . . . . . . . 53

2.4 Long Term Evolution . . . . . . . . . . . . . . . . . . . . . 54

2.4.1 OFDM Transmitter Chain . . . . . . . . . . . . . . . 57

2.4.2 LTE Downlink Radio Frame . . . . . . . . . . . . . . . . . 64

2.4.3 Synchronization Signals . . . . . . . . . . . . . . . . . . . 64

2.4.4 Reference Signals and Antenna Ports _ . . . . . . . . . . . 67

2.4.5 Channel Estimation . . . . . . . . . . . . . . . . . . . . 73

2.4.6 LTE Link Adaptation . . . . . . . . . . . . . . . . . . . . . 79

2.5 LTE Transmission Modes . . . . . . . . . . . . . . . . . . . . . . 82

2.5.1 TM1 Single Antenna Transmission . . . . . . . . . . 83

2.5.2 TM2 Transmit Diversity . . . . . . . . . . . . . . . . . . 84

2.5.3 TM3 Open-Loop Spatial Multiplexing . . . . . . . . . . . . . 90

2.5.4 TM4 Closed-Loop Spatial Multiplexing . . . . . . . . . . . . . . 102

2.5.5 TM6 Single Layer Closed-Loop Spatial Multiplexing . . . . . . . 109 
3 Performance Simulation Setup $\quad 111$

3.1 Link Level Simulator . . . . . . . . . . . . . . . . . . . . . . . . . 111

3.1.1 Downlink Channel Modeling in the Link Level Simulator _ . . . . 111

3.1.2 Channel Models used in the Simulator . . . . . . . . . . . . 118

3.2 Simulating the LTE Downlink using the Link Level Simulator … . . 120

3.3 Performance Measurements and Channel Quality Indicators _ . . . . . 120

3.3.1 Throughput Measurement . . . . . . . . . . . . . . 120

3.3.2 CQI and Rank Measurement . . . . . . . . . . . . . . . . . . . . . 121

3.4 Simulation Parameters . . . . . . . . . . . . . . . . . . . . . . . 122

3.4.1 System Parameters . . . . . . . . . . . . . . . . . . . . 122

3.4.2 Transmission Parameters . . . . . . . . . . . . . . . . . . 124

3.4.3 Simulation Duration . . . . . . . . . . . . . . 126

3.4.4 Antenna Simulation Parameters . . . . . . . . . . . . . . 126

3.4.5 Channel Simulation Parameters . . . . . . . . . . . . . 128

3.4.6 Resource Allocation and Scheduling . . . . . . . . . . . . . . 131

3.4.7 Receiver Algorithms . . . . . . . . . . . . . . . . . . . . . . 132

3.4.8 Channel State Information (CSI) Reporting for Link Adaptation (LA) 133

3.5 Performance Benchmarks . . . . . . . . . . . . . . . . . 135

3.5.1 Throughput Performance Benchmarking . . . . . . . . . . 135

3.5.2 UE Velocity Benchmarking . . . . . . . . . . . . . 135

4 Simulation Results 137

4.1 Antenna Configuration Performance . . . . . . . . . . . . . 137

4.1.1 Base Station Antenna Configurations _. . . . . . . . . . . 138

4.1.2 Mobile Station Antenna Configuration . . . . . . . . . . . . . . 141

4.1.3 Summary of the Antenna Configurations . . . . . . . . . . . . 142

4.1.4 Transmission Modes for Antenna Configuration Simulations . . . 142

4.1.5 4-Tx 4-Port TM2 vs TM4 rank-1 Throughput Differences at High and Low Antenna Correlation using SCME and EPA Channel Models 144

4.1.6 Number of Antennas and Antenna Port Simulations . . . . . . . . 155

4.2 Phase Noise Effects on the LTE Downlink . . . . . . . . . . . . 170

4.2.1 Phase Noise Model . . . . . . . . . . . . . . . . . . . . . . 173

4.2.2 Reference Simulations using Generated Phase Noise and the 3GPP EPA Channel Model . . . . . . . . . . . . . . . . . . . 175

4.2.3 Measured Phase Noise Simulations . . . . . . . . . . . . . . 179 
4.3 Effects of UE Velocity and Feedback Reporting on The LTE Downlink . . . 195

4.3.1 Practical Issues of the Link Adaptation Control Loop . . . . . . . . 195

4.3.2 LTE Downlink Throughput vs CSI Reporting Settings and UE Mobility Simulations . . . . . . . . . . . . . . 201

4.3.3 LTE Downlink Simulations at High Speed Train Velocities . . . . . 209

4.3.4 High Speed Train Results and Discussion . . . . . . . . . . . . 211

5 Conclusion and Future Work 220

5.1 Conclusion . . . . . . . . . . . . . . . . 220

5.2 Future Work . . . . . . . . . . . . . . . . . . 222

$\begin{array}{lr}\text { Appendix A } & 225\end{array}$

A.1 4-bit Channel Quality Indicator Table . . . . . . . . . . . . 225

A.2 Modulation and Transport Block Size Index Table for the PDSCH . . . . . 225

A.3 Resource Block Group Size versus Downlink Bandwidth (Type 0 Resource Allocation) . . . . . . . . . . . . . . . 225

$\begin{array}{lr}\text { Appendix B } & 229\end{array}$

B.1 Jakes' Model . . . . . . . . . . . . . . . . . . . . . . . . . . 229

$\begin{array}{ll}\text { Appendix C } & 231\end{array}$

C.1 Peak Theoretical Throughput for LTE Downlink Transmissions . . . . . . . 231

C.1.1 Peak Theoretical Throughput for 8 PRB UE Allocation using Single Codeword Transmissions . . . . . . . . . . . . . . 231

C.1.2 Table of Peak Theoretical Throughput Values for Various Configurations used in this Thesis . . . . . . . . . . . . . . 232

Appendix D

D.1 Correlation Matrices for 3GPP Conformance Testing Channel Models . . . 234

List of References 


\section{List of Tables}

1.1 LTE Uplink/Downlink Throughput Speeds versus User Equipment Categories 2

2.1 Feature Comparison for SCM, SCME, WIM1, WIM2 Spatial Channel

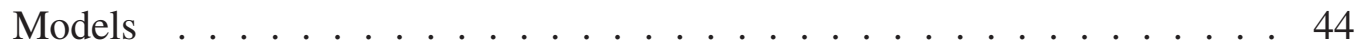

2.2 Environment Parameters for the Urban Macro Scenario . . . . . . . . . . 48

2.3 Description of the Spatial Channel Model Angular Parameters . . . . . . . 50

2.4 Description of the Parameters used in the SCME's Channel TDL Generating Equation . . . . . . . . . . . . . . . . . . . . 52

2.5 Typical LTE System Parameters . . . . . . . . . . . . . . . . 56

2.6 Types of Downlink Reference Signals Defined in 3GPP LTE Rel 8 . . . . . 68

2.7 Coherence Bandwidth for 3GPP Conformance Testing Channel Models . 77

2.8 Channel State Information Reported by the UE . . . . . . . . . . . . . 81

2.9 LTE Transmission Modes . . . . . . . . . . . . . . . . . 83

2.10 Codeword-to-Layer Mapping for Transmit Diversity (2 Antenna Ports) . . . 86

2.11 Codeword-to-Layer Mapping for Transmit Diversity (4 Antenna Ports) . . . 88

2.12 TM3 PDSCH Transmission Scheme . . . . . . . . . . . . . . . . . 91

2.13 Sub-Band Size vs. System Bandwidth . . . . . . . . . . . . . . . 93

2.14 Downlink Physical Layer Parameters Set By Parameter UE-category . . . . 94

2.15 Downlink Physical Layer Codeword-to-Layer Mapping . . . . . . . . . . . 94

2.16 Precoder Codebook for Transmission on Two Antenna Ports . . . . . . . . 98

2.17 Precoder Codebook for Transmission on Four Antenna Ports From [1] . . . 101

2.18 TM4 PDSCH Transmission Scheme . . . . . . . . . . . . . . . . 103

3.1 Base Simulation Parameters for LTE Downlink Throughput Comparisons . 123

3.2 System Bandwidth used in Each Study . . . . . . . . . . . . . . . . 124

3.3 Transmission Modes used in Each Study . . . . . . . . . . . . . . . 125

3.4 Antenna Ports used in Each Study . . . . . . . . . . . . . . . 125

3.5 Reference Signal Power Boosting used in Each Study . . . . . . . . . . . 126

3.6 Channel Models used in Each Study . . . . . . . . . . . . . . . . . . 129 
3.7 UE Velocities used in this Thesis . . . . . . . . . . . . . . . . . . 130

3.8 Spectral Resources Allocated to the UE in Each Study . . . . . . . . . . 131

3.9 Outer-loop Link Adaptation Settings used in Each Study . . . . . . . . . 132

3.10 Link Adaptation Settings used in Each Study . . . . . . . . . . . 134

3.11 Sub-band CQI/PMI Group Size Settings used in Each Study . . . . . . 134

4.1 Wavelengths and Antenna Element Spacing of Two Common LTE Frequency Bands . . . . . . . . . . . . . . . . . . . . . 142

4.2 BS and MS Antenna Configurations . . . . . . . . . . . . 143

4.3 Simulation Parameters for 4-Tx 4-Port TM2 and TM4 Rank-1 Simulations using the SCME and EPA Channel Models . . . . . . . . . . . . . . . 147

4.4 Simulated vs Theoretical Throughput for 4-Tx 4-port TM2 and TM4 rank-1 with Highly Correlated Cross-Polarized Antennas at $3 \mathrm{~km} / \mathrm{hr}$. . . . . . . 148

4.5 Simulated vs Theoretical Throughput for 4-Tx 4-port TM2 and TM4 rank-1 with Uncorrelated Cross-Polarized Antennas at $3 \mathrm{~km} / \mathrm{hr}$. . . . . . . . . 148

4.6 Throughput of 4-Tx 4-Port TM2 and TM4 rank-1 at $10 \mathrm{~dB}$ SNR (EPA) . . 149

4.7 Throughput of 4-Tx 4-Port TM2 and TM4 rank-1 at $10 \mathrm{~dB}$ SNR (SCME) . 149

4.8 Gain of 4-Tx 4-Port TM4 rank-1 Over Other Modes and Antenna Config-

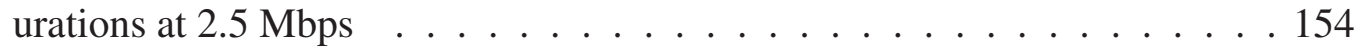

4.9 Simulation Parameters for the Antenna Configuration Simulations . . . . 157

4.10 Gain of 4 Transmitters 4-Port TM4 Over Other Number of Antennas at 25 Mbps at a UE Velocity of $2.1 \mathrm{~km} / \mathrm{hr} \ldots \ldots \ldots$. . . . . . . . . . . . . .

4.11 Gain of 4 Transmitters 4-Port TM4 Over Other Number of Antennas at 25 Mbps at a UE Velocity of $20.8 \mathrm{~km} / \mathrm{hr} \ldots \ldots \ldots \ldots . \ldots \ldots$

4.12 Throughput of Best Antenna Configuration at $14 \mathrm{~dB}$ SNR $(2.1 \mathrm{~km} / \mathrm{hr}) \ldots 166$

4.13 Simulation Parameters for the Generated Phase Noise Simulations . . . . 177

4.14 Phase Jitter Data Set Statistics ．. . . . . . . . . . . . . . . . . 185

4.15 Simulation Parameters for the Measured Phase Noise Simulations . . . . 187

4.16 Approximate Maximum UE Velocity Supported by CQI Reporting . . . . . 200

4.17 Simulation Parameters for the Downlink Throughput vs CSI Reporting Settings and UE Velocity Simulations . . . . . . . . . . . . . 203

4.18 Simulation Parameters for the LTE Downlink Throughput in the High Speed Train Scenario . . . . . . . . . . . . . . . . . . 212

4.19 Downlink Throughput at Increasing UE Velocity . . . . . . . . . . . 213

4.20 Average CQI Index at Increasing UE Velocity . . . . . . . . . . . . 218 
4.21 Distribution of Rank 2 Transmissions at Increasing UE Velocity . . . . . . 219

A.1 4-bit CQI Table . . . . . . . . . . . . . . . 226

A.2 Modulation and Transport Block Size (TBS) Index Table for the Physical Down-link Shared Channel $(\mathrm{PDSCH})$. . . . . . . . . . . . . . . . 227

A.3 Resource Block Group Size vs. Downlink Bandwidth (Type 0 Resource Allocation) . . . . . . . . . . . . . . . . . . . 228

C.1 Peak Theoretical Throughput Values for Various Transmission Settings using the LTE Transport Block Size Table . . . . . . . . . . . . . . . . . 233

D.1 Extended-ITU Spatial Correlation Parameters $\alpha$ and $\beta \ldots . . . . . .235$ 


\section{List of Figures}

1.1 The Wireless Radio Access Network and Wireless Channel . . . . . . . . . 3

1.2 The Protocol Stack and Data Flow in an LTE Network . . . . . . . . . 3

1.3 Scattering due to Angle Spread at the BS . . . . . . . . . . . . . 7

2.1 Multi-Path Propagation . . . . . . . . . . . . . . 16

2.2 Channel Impulse Response $h(t, \tau)$ at time $t \ldots \ldots \ldots$

2.3 Slow and Fast Rayleigh Fading . . . . . . . . . . . . . . . . . 18

2.4 Frequency Flat Channel . . . . . . . . . . . . . . . . . . . 20

2.5 Frequency Selective Channel . . . . . . . . . . . . . . . . . 21

2.6 OFDM Sub-Carriers with Frequency Domain Spacing $\Delta f \ldots \ldots 22$

2.7 OFDM Cyclic Prefix with Inter-Symbol Interference . . . . . . . . . . . 24

2.8 SISO Channel Model and Channel Impulse Response . . . . . . . . . . . . 26

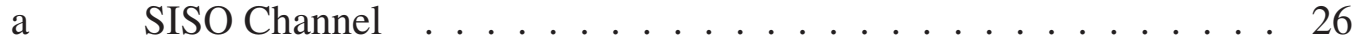

b Channel Impulse Response for the SISO Channel . . . . . . . . . . 26

2.9 MIMO Channel . . . . . . . . . . . . . . . . . . . . . 27

2.10 MIMO Channel Impulse Responses . . . . . . . . . . . . . . . . 27

2.11 Conversion of the MIMO Channel to Parallel Gaussian Channels using Singular Value Decomposition (SVD) . . . . . . . . . . . . . . . . . 29

a Pre and Post-Processing Matrices Applied to MIMO Channel . . . 29

b Resulting Parallel Gaussian Channels After Application of SVD

Pre and Post-Processing Matrices . . . . . . . . . . . . . . . . 29

2.12 The Received Electric Field using the Ray Model . . . . . . . . . . . . . . 32

2.13 A uniformly distributed set of incident wave fronts, with angle of arrivals $\alpha_{n}$, arriving at the UE . . . . . . . . . . . . 36

2.14 Illustration of the Jakes U-Shaped Doppler Spectrum . . . . . . . . . . . 37

2.15 Diagram of Jakes' model . . . . . . . . . . . . . . . . . . . . . 39

2.16 Comparison from figure 1 of [2] of the Autocorrelation of the Improved SoS Simulator Proposed in [2] vs the Reference Model . . . . . . . . . . . 42 
2.17 The Drop (or Channel Segment) _ . . . . . . . . . . . . . . . . 45

2.18 Diagram from [3] of the Sub-paths and Clusters used in the Spatial Channel Model . . . . . . . . . . . . . . . . . . . . . . . . . . . . 49

2.19 Spatial Channel Model . . . . . . . . . . . . . . . . . . . . . 52

2.20 Simulation Model: Channel Generation and Spatial Channel Parameters Used to Model a SISO Link . . . . . . . . . . . . . . . . . . . 55

2.21 LTE OFDM Transmitter Chain with Modulation and Coding, IFFT and CP Addition for Single Layer Transmissions . . . . . . . . . . . . . . 57

2.22 LTE OFDM Transmitter Chain with Layer Mapping, Precoding and Port

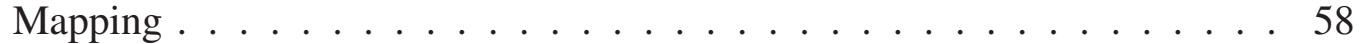

2.23 Block Diagram of the LTE Turbo Encoder . . . . . . . . . . . . . . . . 59

2.24 Illustration of the LTE Turbo Encoding with the Circular Buffer for Rate

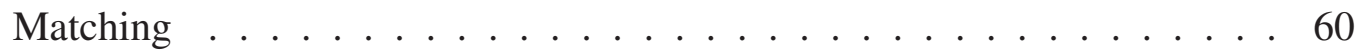

2.25 IQ Modulator and RF Up-Conversion . . . . . . . . . . . . . 63

2.26 Decomposition of the LTE Frame into Sub-frames and Slots . . . . . . 64

2.27 LTE Resource Map From Figure 6.2.1-1 of [1] . . . . . . . . . . . . . 65

2.28 Resource Map Showing PSS, SSS and RS . . . . . . . . . . . . . . 66

2.29 Cell-Specific Reference Signal Patterns for 2-Tx 2-Ports . . . . . . . . . 70

2.30 Cell-Specific Reference Signal Patterns for 4-Tx 2-Ports . . . . . . . . . 71

2.31 Cell-Specific Reference Signal Patterns for 4-Tx 4-Ports . . . . . . . . 72

2.32 Downlink Cell-Specific Reference Signal Pattern $\left(R S_{0}\right.$ for Port 0$) \quad \ldots$

2.33 Downlink Reference Signal Correlation in Low Delay Spread or Low Mobility Scenarios . . . . . . . . . . . . . . . 76

$2.342 \times 1$ 1D Channel Estimate Interpolation . . . . . . . . . . . . . 78 a Frequency Domain Filtering Pass For Channel Estimation . . . . 78 b Time Domain Filtering Pass For Channel Estimation . . . . . . . 78

2.35 Frequency Domain Representation of Sub-bands and Sub-band CQI Re-

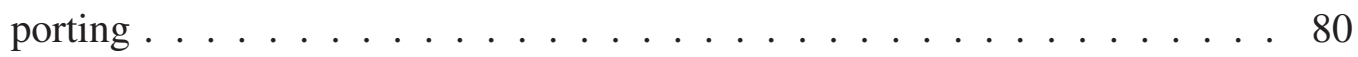

2.36 Single Antenna Port Transmission _. . . . . . . . . . . . . . 84

2.37 Transmit Diversity SFBC Transmissions on 2 Antennas Ports . . . . . . . . 86

2.38 Transmit Diversity SFBC-FSTD Transmissions using 4 Antenna Ports . . . 88

2.39 Open-Loop Spatial Multiplexing (OLSM) ～. . . . . . . . . . . . . . 90

2.40 Addition of CDD to Transmitter Chain and Resulting Sub-Carrier Level Beamforming . . . . . . . . . . . . . . . . . . 97 
2.41 Beamforming Patterns for a Two Antenna Base Station Configuration . . . 99 a Beamforming Patterns using a Single Transmission Layer . . . . . 99

b Beamforming Patterns using Two Transmission Layers . . . . . . . 99

2.42 Closed-Loop Spatial Multiplexing (CLSM) . . . . . . . . . . . . . 102

2.43 Single-Layer Closed-Loop Spatial Multiplexing (Codebook Based Beamforming . . . . . . . . . . . . . . . . . 110

3.1 User Input Parameters for the Link Level Simulator . . . . . . . . . . . . . 112

3.2 The Simulation Scenario used with the Extended Spatial Channel Model $(\mathrm{SCME}) \ldots \ldots \ldots \ldots 114 \ldots \ldots \ldots$

4.1 Co-polarized BS Antenna Element Configurations . . . . . . . . . . . . . 140

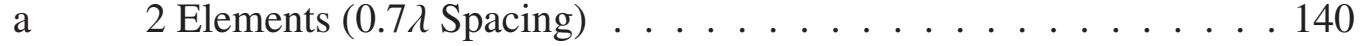

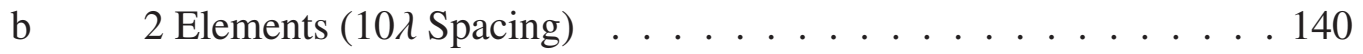

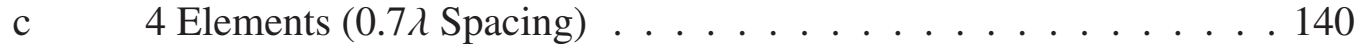

d 4 Elements (10 $\lambda$ Spacing) . . . . . . . . . . . . . . 140

4.2 Cross-Polarized BS Antenna Element Configurations . . . . . . . . . . . . 140

a 2 Elements ..................... . . 140

b 4 Elements $(0.7 \lambda$ Spacing $) \ldots \ldots$. . . . . . . . . 140

c 4 Elements (10ג Spacing) . . . . . . . . . . . . . . 140

4.3 MS Antenna Configurations . . . . . . . . . . . . . . . . 141

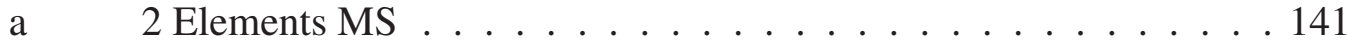

b 2 Element Laptop . . . . . . . . . . . . . . . . . . . . . 141

c 4 Elements Laptop . . . . . . . . . . . . . . . . . . . . . . 141

4.4 4-Tx 4-Port TM2 and TM4 rank-1 EPA and SCME Downlink Throughput using Cross-polarized Antennas at $3 \mathrm{~km} / \mathrm{hr}$. . . . . . . . . . . . . . . . 151

a 4-Tx 4-port TM2 and TM4 rank-1 EPA and SCME Throughput using Correlated Antennas . . . . . . . . . . . . . . . . . . . 151

b 4-Tx 4-port TM2 and TM4 rank-1 EPA and SCME Throughput using Uncorrelated Antennas . . . . . . . . . . . . . . 151

4.5 4-Tx 4-Port TM2 and TM4 rank-1 EPA and SCME Downlink Throughput using Cross-polarized Antennas at $30 \mathrm{~km} / \mathrm{hr}$. . . . . . . . . . . . . 152

a 4-Tx 4-port TM2 and TM4 rank-1 EPA and SCME Throughput using Correlated Antennas . . . . . . . . . . . . . . . . . . . 152

b 4-Tx 4-port TM2 and TM4 rank-1 EPA and SCME Throughput using Uncorrelated Antennas . . . . . . . . . . . . . . . . . . . 152 
4.6 4-Tx 4-Port TM2 and TM4 rank-1 EPA and SCME Downlink Throughput using Cross-polarized Antennas at $120 \mathrm{~km} / \mathrm{hr}$. . . . . . . . . . . . . . 153

a 4-Tx 4-port TM2 and TM4 rank-1 EPA and SCME Throughput using Correlated Antennas . . . . . . . . . . . . . . . . 153

b 4-Tx 4-port TM2 and TM4 rank-1 EPA and SCME Throughput using Uncorrelated Antennas . . . . . . . . . . . . . . . . 153

4.7 LTE DL Performance versus Number of Antennas and Ports at Low Velocity at a UE Velocity of $2.1 \mathrm{~km} / \mathrm{hr}$. . . . . . . . . . . . . . . . . . . . . 159

a Correlated $(0.7 \lambda)$ Cross-polarized Antennas $(2.1 \mathrm{~km} / \mathrm{hr}) \quad \ldots . . .159$

b Uncorrelated $(10 \lambda)$ Co-polarized Antennas $(2.1 \mathrm{~km} / \mathrm{hr}) \ldots 159$

4.8 LTE DL Performance versus Number of Antennas and Ports at a UE Velocity of $2.1 \mathrm{~km} / \mathrm{hr} \ldots \ldots \ldots$

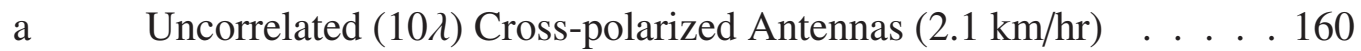

b Correlated $(0.7 \lambda)$ Co-polarized Antennas $(2.1 \mathrm{~km} / \mathrm{hr})$. . . . . . . . 160

4.9 LTE DL Performance versus Number of Antennas and Ports at a UE Velocity of $20.8 \mathrm{~km} / \mathrm{hr} \ldots \ldots \ldots$. . . . . . . . . . . . . . . . . . . . . . . . . . . .

a Correlated $(0.7 \lambda)$ Cross-polarized Antennas $(20.8 \mathrm{~km} / \mathrm{hr}) \ldots . . .163$

b Uncorrelated $(10 \lambda)$ Co-polarized Antennas $(20.8 \mathrm{~km} / \mathrm{hr}) \ldots . . .163$

4.10 LTE DL Performance versus Number of Antennas and Ports at a UE Ve-

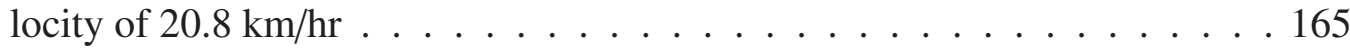

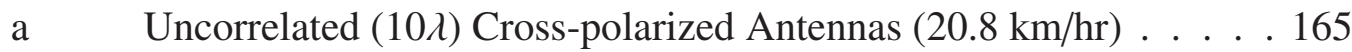

b Correlated (0.7 $\lambda)$ Co-polarized Antennas $(20.8 \mathrm{~km} / \mathrm{hr})$. . . . . . 165

4.11 Comparison of 4-Tx 4-Port Polarization and Antenna Correlation . . . . . . 168 a Cross-polarized vs Co-Polarized Antennas $(2.1 \mathrm{~km} / \mathrm{hr}) \ldots . . .168$

b Cross-polarized vs Co-Polarized Antennas $(20.8 \mathrm{~km} / \mathrm{hr})$. . . . . 168

4.12 Best Performing Antenna Configuration for Each Transmission Mode . . 169 a Best Performing Antenna Configuration for Each Transmission Mode $(2.1 \mathrm{~km} / \mathrm{hr})$. . . . . . . . . . . . . . . . . . . . . 169

b Best Performing Antenna Configuration for Each Transmission Mode $(20.8 \mathrm{~km} / \mathrm{hr})$. . . . . . . . . . . . . . . . . . . . . . . 169

4.13 Ideal versus Practical Oscillator . . . . . . . . . . . . . . . . . . 171

4.14 Error Floors and Throughput Ceilings due to ICI . . . . . . . . . . 174

4.15 Phase Noise Effect on the LTE Downlink using TM2 and TM4 rank-1 and the EPA Channel Model with High and Low Antenna Correlation at $3 \mathrm{~km} / \mathrm{hr} 178$ 
a EPA, High Antenna Correlation, UE Velocity $3 \mathrm{~km} / \mathrm{hr} \ldots 178$

b EPA, Low Antenna Correlation, UE Velocity 3 km/hr . . . . . . . . 178

4.16 Phase Noise Effect on the LTE Downlink using TM2 and TM4 rank-1 and the EPA Channel Model with High and Low Antenna Correlation at $30 \mathrm{~km} / \mathrm{hr} 180$ a EPA, High Antenna Correlation, UE Velocity $30 \mathrm{~km} / \mathrm{hr}$. . . . . . 180

b EPA, Low Antenna Correlation, UE Velocity $30 \mathrm{~km} / \mathrm{hr}$. . . . . . 180

4.17 Phase Noise Measurement Between Ports . . . . . . . . . . . . . . . . 181

4.18 Phase Noise Data Set $\theta_{1}(t) \ldots \ldots \ldots$. . . . . . . . . . 182

4.19 Spectrum of Phase Noise Data Set $\theta_{1}(t) \ldots \ldots$. . . . . . . . . . 183

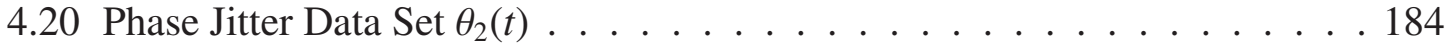

4.21 Spectrum of Phase Noise Data Set $\theta_{2}(t) \quad \ldots \ldots$. . . . . . . . . 184

4.22 BS Antenna Port Connections for Phase Noise Simulations . . . . . . . . 188

a Cross-Polarized Antennas (Co-Pol on Different Radios) . . . . . 188

b Linear Array (Adjacent Elements on Different Radios) . . . . . . . 188

4.23 Phase Jitter Impairment Results on the LTE FDD Downlink in $20 \mathrm{MHz}$ of Bandwidth using Cross-polarized BS Antennas and TM2 vs TM4 rank1 . . 189 a Cross-Pol Antenna Spacing $0.7 \lambda$, UE Velocity 3 km/hr . . . . . . . 189

b Cross-Pol Antenna Spacing 10 , UE Velocity $3 \mathrm{~km} / \mathrm{hr}$. . . . . . . 189

4.24 Phase Jitter Impairment Results on the LTE FDD Downlink in $20 \mathrm{MHz}$ of Bandwidth using Co-Polarized BS Antennas and TM2 vs TM4 rank1 . . . . 190 a Co-Pol Antenna Spacing 0.7 , UE Velocity $3 \mathrm{~km} / \mathrm{hr}$. . . . . . . 190

b Co-Pol Antenna Spacing 10ג, UE Velocity 3 km/hr . . . . . . . . . 190

4.25 Phase Jitter Impairment Results on the LTE FDD Downlink in $20 \mathrm{MHz}$ of Bandwidth using Cross-polarized BS Antennas and TM2 vs TM4 rank1 . . 192 a Cross-Pol Antenna Spacing 0.7 , UE Velocity 120 km/hr . . . . . . 192

b Cross-Pol Antenna Spacing 10ג, UE Velocity $120 \mathrm{~km} / \mathrm{hr}$. . . . . 192

4.26 Phase Jitter Impairment Results on the LTE FDD Downlink in $20 \mathrm{MHz}$ of Bandwidth using Cross-polarized BS Antennas and TM2 vs TM4 rank1 . . 193 a Co-Pol Antenna Spacing 0.7 $\lambda$, UE Velocity $120 \mathrm{~km} / \mathrm{hr}$. . . . . . . 193

b Co-Pol Antenna Spacing 10ג, UE Velocity $120 \mathrm{~km} / \mathrm{hr}$. . . . . . . 193

4.27 CQI Measurement, Feedback and Application Cycle (10 msec reporting interval) when using Radio Frame Type 1 (FDD) . . . . . . . . . . . . . . 199

4.28 4-Tx 4-Port Throughput vs UE Velocity at $15 \mathrm{~dB}$ SNR for 3 Reporting Intervals . . . . . . . . . . . . . . . . . . 206 
a Throughput vs Velocity at $15 \mathrm{~dB}$ SNR using Near Ideal CSI (CQI Delay $=2 \mathrm{msec}$ ) . . . . . . . . . . . . 206

b Throughput vs Velocity at $15 \mathrm{~dB}$ SNR using Aged CSI (CQI Delay $=8 \mathrm{msec}$. . . . . . . . . . . . . . 206

4.29 4-Tx 2-Port Throughput vs UE Velocity at $15 \mathrm{~dB}$ SNR for 3 Reporting Intervals . . . . . . . . . . . . . . . . . . . . 207

a Throughput vs Velocity at $15 \mathrm{~dB}$ SNR using Near Ideal CSI (CQI Delay $=2 \mathrm{msec}$ ) . . . . . . . . . . . . . . 207

b Throughput vs Velocity at $15 \mathrm{~dB}$ SNR using Aged CSI (CQI Delay $=8 \mathrm{msec}$. . . . . . . . . . . . . . . . . 207

4.30 2-Tx 2-Port Throughput vs UE Velocity at $15 \mathrm{~dB}$ SNR for 3 Reporting Intervals . . . . . . . . . . . . . . . . . . . . . 208

a Throughput vs Velocity at $15 \mathrm{~dB}$ SNR using Near Ideal CSI (CQI Delay $=2 \mathrm{msec}) \ldots \ldots$. . . . . . . . . . . . . 208

b Throughput vs Velocity at $15 \mathrm{~dB}$ SNR using Aged CSI (CQI Delay $=8 \mathrm{msec}$. . . . . . . . . . . . . . . 208

4.31 High Speed Train Doppler Profiles from [4] . . . . . . . . . . . . . . . . 210

a Doppler Profile for Scenario 1 "Open Space" . . . . . . . . . . . 210

b Doppler Profile for Scenario 3 "Tunnel" . . . . . . . . . . . . . . . 210

4.32 High Speed Train Physical Scenario for Simulation . . . . . . . . . . . 213

4.33 4-Tx 4-Port High Speed Train Simulation Results . . . . . . . . . . . . . . 214

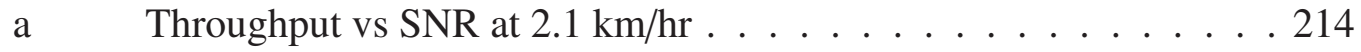

b Rank Distribution and Average CQI MCS Index at $2.1 \mathrm{~km} / \mathrm{hr}$. . . . 214

4.34 4-Tx 4-Port High Speed Train Simulation Results at 124.6 km/hr . . . . . . 215

a Throughput vs SNR at $124.6 \mathrm{~km} / \mathrm{hr} \ldots \ldots . \ldots . . \ldots 215$

b Rank Distribution and Average CQI MCS Index at $124.6 \mathrm{~km} / \mathrm{hr}$. . 215

4.35 4-Tx 4-Port High Speed Train Simulation Results at 200 km/hr . . . . . . 216

a Throughput vs SNR at $200 \mathrm{~km} / \mathrm{hr} \ldots \ldots . \ldots . \ldots . \ldots$

b Rank Distribution and Average CQI MCS Index at $200 \mathrm{~km} / \mathrm{hr}$. . . 216

4.36 4-Tx 4-Port High Speed Train Simulation Results at 350 km/hr . . . . . . . 217

a Throughput vs SNR at $350 \mathrm{~km} / \mathrm{hr} \ldots \ldots . . \ldots 217$

b Rank Distribution and Average CQI MCS Index at $350 \mathrm{~km} / \mathrm{hr}$. . . 217 


\section{List of Symbols}

$N_{I D}^{(2)}$ Cell identity group

$N_{\text {Data } R E S}^{S F}$ Number of available data (RE) per sub-frame (SF) (for normal sub-frames $\{1,2,3,4,6,7,8,9\})$

$N_{P D C C H R E}^{S F}$ Number of control channel (PDCCH) resource elements (RE) per sub-frame (SF)

$N_{P D C C H}^{S F}$ sym Number of control channel (PDCCH) OFDM symbols per sub-frame (SF)

$N_{R E}^{S F}$ Total number of resource elements (RE) per sub-frame (SF)

$N_{R S}^{P D C C H}$ Number of reference signals (RS) per sub-frame (SF) that fall in the PDCCH

$N_{R S}^{S F}$ Number of reference signals (RS) per sub-frame (SF)

$N_{S y m b}^{S F}$ Number of OFDM symbols (Symb) per sub-frame (SF)

$N_{s c}^{R B}$ Total number of sub-carriers (SC) per resource block (RB)

$R_{\max }$ Maximum code rate

$B_{c}$ Coherence bandwidth of the channel

$B_{s}$ OFDM sub-carrier bandwidth

$B_{c, 50 \%} 50 \%$ coherence bandwidth of the channel

$B_{c, 90 \%} 90 \%$ coherence bandwidth of the channel

$E\left(\sigma_{A S}\right)$ Mean angle spread at the BS

$E\left(\sigma_{A S}, M S\right)$ Mean angle spread at the MS 
$E\left(\sigma_{D S}\right)$ Mean total RMS delay spread

$I_{M C S}$ Modulation index

$I_{T B S}$ Transport block size index

$N_{R B}^{D L}$ Number of resource blocks in the down-link

$N_{P R B}$ Number of physical resource blocks allocated to the scheduled UE

$P_{n}$ Power delay profile $n^{\text {th }}$ average path power

$Q_{m}$ Number of modulation bits

$T_{s}$ OFDM symbol period

$T_{c, 50 \%} 50 \%$ Coherence time

$T_{c p}$ Length of the cyclic prefix in seconds

$T_{c}$ Coherence time

$T_{d}$ Delay spread of the channel

$\Delta_{n, m, A o A}$ The offset for the $m^{\text {th }}$ path sub-path of the $n^{\text {th }}$ path with respect to $\delta_{n, A o A}$

$\Delta_{n, m, A o D}$ The offset for the $m^{\text {th }}$ path sub-path of the $n^{\text {th }}$ path with respect to $\delta_{n, A o D}$

$\Delta f$ OFDM sub-carrier frequency domain spacing

$\Delta f$ Sub-carrier spacing in an OFDM system

$\Omega_{B S}$ The BS array broadside orientation with respect to absolute North

$\Omega_{M S}$ The MS array orientation with respect to absolute North

$\Phi_{n, m}^{(x, y)}$ Phase of the $m^{\text {th }}$ sub-path of the $n^{\text {th }}$ path between the $x$ component of the BS antenna element and the $y$ component of the MS antenna element

$\mathbf{R}_{\mathbf{B S}}$ The spatial correlation matrix at the BS

$\mathbf{R}_{\mathrm{UE}}$ The spatial correlation matrix at the UE

$\mathbf{R}_{\text {spat }}$ The spatial correlation matrix of the system 
$\chi_{B S}^{(h)}\left(\theta_{n, m, A o D}\right)$ BS antenna complex response for the horizontal polarization component

$\chi_{B S}^{(v)}\left(\theta_{n, m, A o D}\right)$ BS antenna complex response for the vertical polarization component

$\chi_{M S}^{(h)}\left(\theta_{n, m, A o A}\right)$ MS antenna complex response for the horizontal polarization component

$\chi_{M S}^{(v)}\left(\theta_{n, m, A o A}\right)$ MS antenna complex response for the vertical polarization component

$\delta_{n, A o A}$ The angle of arrival for the $n^{\text {th }}$ path with respect to the LOS AoA $\theta_{M S}$

$\delta_{n, A o D}$ The angle of departure for the $n^{\text {th }}$ path with respect to the $\operatorname{LOS} \operatorname{AoD} \theta_{B S}$

$\sigma_{A S}$ Angle spread at the base-station as a log-normal random variable

$\sigma_{A o D}$ The standard deviation of log-normal random variable of the angle spread at the base-station

$\sigma_{D S}$ Delay spread as log-normal random variable

$\sigma_{S F}$ Standard deviation of the channel log-normal shadowing

$\tau_{n}$ Power delay profile $n^{\text {th }}$ path delay

$\theta_{B S}$ The angle between the BS-MS LOS and the BS array broadside

$\theta_{M S}$ The angle between the BS-MS LOS and the MS antenna broadside

$\theta_{n, m, A o A}$ The absolute AoA for the $m^{\text {th }}$ sub-path of the $n^{\text {th }}$ path at the MS with respect to the MS array broadside

$\theta_{n, m, A o D}$ The absolute AoD for the $m^{\text {th }}$ sub-path of the $n^{\text {th }}$ path at the BS with respect to the BS array broadside

$\theta_{v}$ The angle of the velocity vector of the MS with respect to the broadside, where $\theta_{v}=$ $\arg (v)$

$\vec{v}$ The MS velocity vector

$d_{s} \mathrm{BS}$ antenna element distance from the $\mathrm{BS}$ antenna array origin (center)

$d_{u}$ MS antenna element distance from the MS antenna array origin (center)

$f_{c}$ Carrier frequency 
$f_{m}$ Maximum Doppler frequency in $\mathrm{Hz}$

$k$ Wave number

$r_{n 1} \mathrm{RV}$ representing the power ratio of waves leaving the $\mathrm{BS}$ along the vertical polarization and arriving at the MS in the horizontal polarization (v-h) to waves arriving in the vertical polarization $(\mathrm{v}-\mathrm{v})$

$r_{n 2} \mathrm{RV}$ representing the power ratio of waves leaving the BS along the horizontal polarization and arriving at the MS in the vertical polarization (h-v) to waves arriving in the horizontal polarization (h-h) 


\section{List of Acronyms}

16QAM 16-ary quadrature amplitude modulation

3G $3^{\text {rd }}$ generation

3GPP $3^{\text {rd }}$ Generation Partnership Project

3GPP2 $3^{\text {rd }}$ Generation Partnership Project 2

4G $4^{\text {th }}$ generation

64QAM 64-ary quadrature amplitude modulation

ACK acknowledgement

ADC analog to digital converter

AGC automatic gain control

AGL above ground level

AMC adaptive modulation and coding

AoA angle of arrival

AoD angle of departure

ARQ automatic repeat request

AS angle spread

AWGN additive white Gaussian noise

B3G beyond 3G systems 
BER bit error rate

BICM bit-interleaved coded modulation

BLER block error rate

BS base station

C-RNTI cell radio network temporary identifier

CAZAC constant amplitude zero autocorrelation

CDD cyclic delay diversity

CDF cumulative distribution function

CDMA code division multiple access

CIR channel impulse response

CLSM closed-loop spatial multiplexing

CoMP coordinated multi-point

CP cyclic prefix

CPE common phase error

CPRI common public radio interface

CQI channel quality indicator

CRC cyclic redundancy check

CRS cell-specific reference signal

CSI channel state information

D/A digital to analog conversion

DAB digital audio broadcast

DAC digital to analog converter 
DC direct current

DCI downlink control information

DFT discrete Fourier transform

DS delay spread

e-UTRAN evolved-universal terrestrial radio access network

EESM effective exponential SNR mapping

eNodeB evolved NodeB

EPA extended pedestrian A

ETU extended typical urban

EVA extended vehicular A

FDD frequency-division multiplexing

FDE frequency domain equilization

FDM frequency division multiplexing

FDMA frequency division multiple access

FEC forward error correction

FFT fast fourier transform

FSS frequency selective scheduling

FSTD frequency-switched transmit diversity

HARQ hybrid automatic repeat request

HS high speed

Hz Hertz

I-Q in-phase and quadrature 
i.i.d. independent and identically distributed

ICI inter-carrier interference

IFFT inverse fast Fourier transform

IMT International Mobile Telecommunications

IMT-Advanced International Mobile Telecommunications - Advanced

IP internet protocol

ISD inter-site distance

ISI inter-symbol interference

ITU International Telecommunication Union

ITU-R International Telecommunication Union - Radio

L1 layer 1

LO local oscillator

LOS line-of-sight

LS least squares

LSP large-scale parameter

LTE Long Term Evolution

LTV linear time variant

M2M machine to machine

MAC media access control

MBSFN multimedia broadcast single frequency network

MBSFN-RS multimedia broadcast single frequency network reference signal

MCS modulation and coding scheme 
MIESM mutual information effective SNR mapping

MIMO multiple input multiple output

ML maximum likelihood

MMSE minimum mean square error

MPC multi-path component

MRC maximal ratio combining

MS mobile station

MSE mean square error

MU-MIMO multi-user MIMO

NACK negative acknowledgment

NLOS non-line-of-sight

OFDM orthogonal frequency division multiplexing

OFDMA orthogonal frequency division multiple access

OLLA outer-loop link adaptation

OLSM open-loop spatial multiplexing

PACE pilot aided channel estimation

PBCH physical broadcast channel

PDCCH physical downlink control channel

PDCP packet data convergence protocol

PDF probability density function

PDSCH physical downlink shared channel

PHY physical layer 
PLL phase-locked loop

PMI precoding matrix indicator

PRB physical resource block

PSS primary synchronization signal

PSTN public switched telephone network

PUCCH physical uplink control channel

PUSCH physical uplink shared channel

QoS quality of service

QPP quadratic permutation polynomial

QPSK quadrature phase shift keying

RAN radio access network

RBG resource block group (a set of consecutive physical resource blocks)

RE resource element

$\mathbf{R F}$ radio frequency

RI rank indicator

RLC radio link control

RMS root mean square

$\mathbf{R S}$ reference signal

RSC recursive systematic convolutional

$\mathbf{R V}$ redundancy version

SCM 3GPP/3GPP2 spatial channel model

SCME spatial channel model extension 
SF shadow fading

SFBC space-frequency block code

SINR signal to interference plus noise ratio

SISO single input single output

SNR signal to noise ratio

SON self organizing network

SoS sum of sinusoids

SSS secondary synchronization signal

SU-MIMO single-user MIMO

SVD singular value decomposition

TBS transport block size

TDD time-division duplexing

TDL tapped-delay-line

TM1 transmission mode 1

TM2 transmission mode 2

TM3 transmission mode 3

TM4 transmission mode 4

TM6 transmission mode 6

TM7 transmission mode 7

TS technical specification

TTI transmission time interval

UE user equipment 
UE-Spec RS UE-specific reference signal

ULA uniform linear array

VCO voltage controlled oscillator

WIM1 WINNER channel model - phase 1

WIM2 WINNER channel model - phase 2

WINNER Wireless World Initiative New Radio

WP1 work package 1

WSS wide sense stationary

XPD cross-polarization discrimination ratio

ZC Zadoff-Chu

ZDSC zero-delay-spread-cluster or zero-delay sub-cluster 


\section{Chapter 1}

\section{Introduction}

Wireless communications is allowing people to remain connected on a daily basis in ways never seen before. Wireless has enhanced our lives in such a way that we can no longer see ourselves without this technology and we can only speculate on what it might enable next. The next generation of wireless networks promise to deliver more bandwidth to the consumer using exciting technologies like multi-antenna wireless systems, Wifi hot spots, pico cells and coordinated multi-point (CoMP) that work with the base station in a wireless radio access network to offload bandwidth from the wireless base stations and increase the consumer experience.

Long Term Evolution (LTE) is a new wireless technology developed by the $3^{\text {rd }}$ Generation Partnership Project (3GPP) and first standardized in 3GPP release 8 standards documentation. In 2007, the first release of LTE offered exciting new improvements to wireless access speeds by using orthogonal frequency division multiplexing (OFDM) on the downlink to send data to the consumer and achieve high bit rates.

The minimum requirements for LTE release 8 set by International Telecommunication Union - Radio (ITU-R) are $\geq 100 \mathrm{Mbps}$ with a peak spectral efficiency of $\geq 5 \mathrm{bps} / \mathrm{Hz}$ for the downlink and $\geq 50 \mathrm{Mbps}$ for the uplink for a reference 2 transmitter by 2 receiver system. The bit rates achievable by the LTE wireless network downlink are shown in table 1.1. The downlink bit rates, also known as throughput, does depend on the capability of the user's mobile device but they are, nonetheless, an impressive step forward in bit rates.

Starting in LTE standard release 10, 3GPP added support for advanced technologies to meet the speed requirements of true $4^{\text {th }}$ generation $(4 \mathrm{G})$ networks set by ITU-R and marketed as International Mobile Telecommunications - Advanced (IMT-Advanced). The IMT-Advanced compatible LTE networks, appropriately named LTE-Advanced, will be 
Table 1.1: LTE Uplink/Downlink Throughput Speeds versus User Equipment Categories

\begin{tabular}{cccccc}
\hline & \multicolumn{5}{c}{ UE Category } \\
& 1 & 2 & 3 & 4 & 5 \\
\hline \hline Downlink speed (Mbps) & 10 & 50 & 100 & 150 & 300 \\
Uplink speed (Mbps) & 5 & 25 & 50 & 50 & 75 \\
Receive antennas required & 2 & 2 & 2 & 2 & 4 \\
Downlink MIMO streams & 1 & 2 & 2 & 2 & 4 \\
\hline
\end{tabular}

capable of downlink speeds of 1 Gbps in low mobility conditions.

\subsection{The Wireless Radio Access Networks}

The wireless radio access network's purpose has changed over the course of the last decade. In the past, the radio access network was predominantly used to make and receive voice phone calls via the public switched telephone network (PSTN). Recently data has become the dominant wireless service as mobile devices allow users to access the Internet, video services and data centric mobile applications. Mobile wireless networks have transitioned from circuit-switched networks and are now becoming all internet protocol (IP) packetswitched networks.

In a wireless radio access network, the base station is the entity which interfaces with the mobile station as seen in figure 1.1. The base station operates within a geographic area, known as a cell, which is generally delimited by the base stations's coverage area. The base station has the authority to perform scheduling, which involves allocating resources in the time and frequency domain to the mobile station in order for data to be transferred to or from the mobile device. The link between the base station and the mobile device is established over the wireless channel and data sent to the mobile device is sent in the "downlink" direction, while data flowing from the mobile device to the base station is sent in the "uplink" direction. 


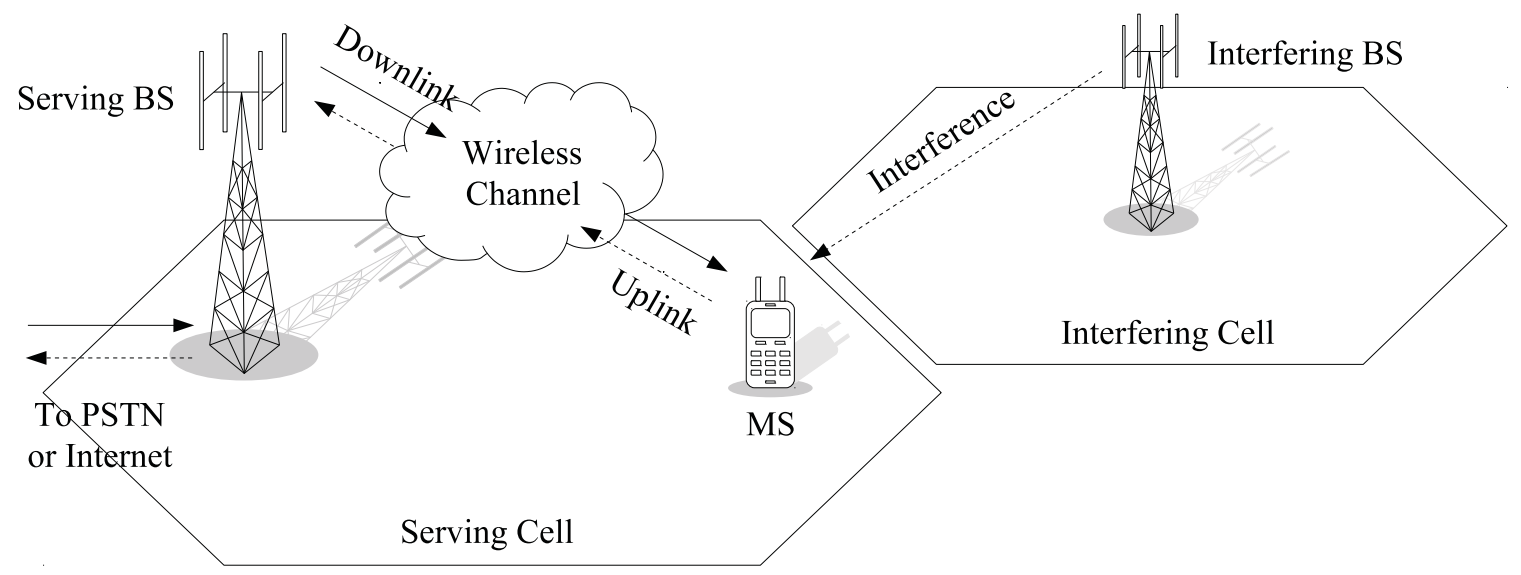

Figure 1.1: The Wireless Radio Access Network and Wireless Channel

\subsection{LTE Radio Air Interface}

The LTE radio access network, known as the evolved-universal terrestrial radio access network (e-UTRAN), is controlled by the base station and the protocol stack which runs in software on the base station hardware. A similar protocol stack residing in the mobile device is designed to interface with the base station protocol stack. Due to the complexity of the protocol stack, the software is implemented based on well defined layers forming the protocol stack, as seen in figure 1.2. The layers highlighted in grey form the e-UTRAN's portion of the protocol stack and enable the seamless exchange of information between the higher layers of the mobile device, base station and application layer at the data's final destination.

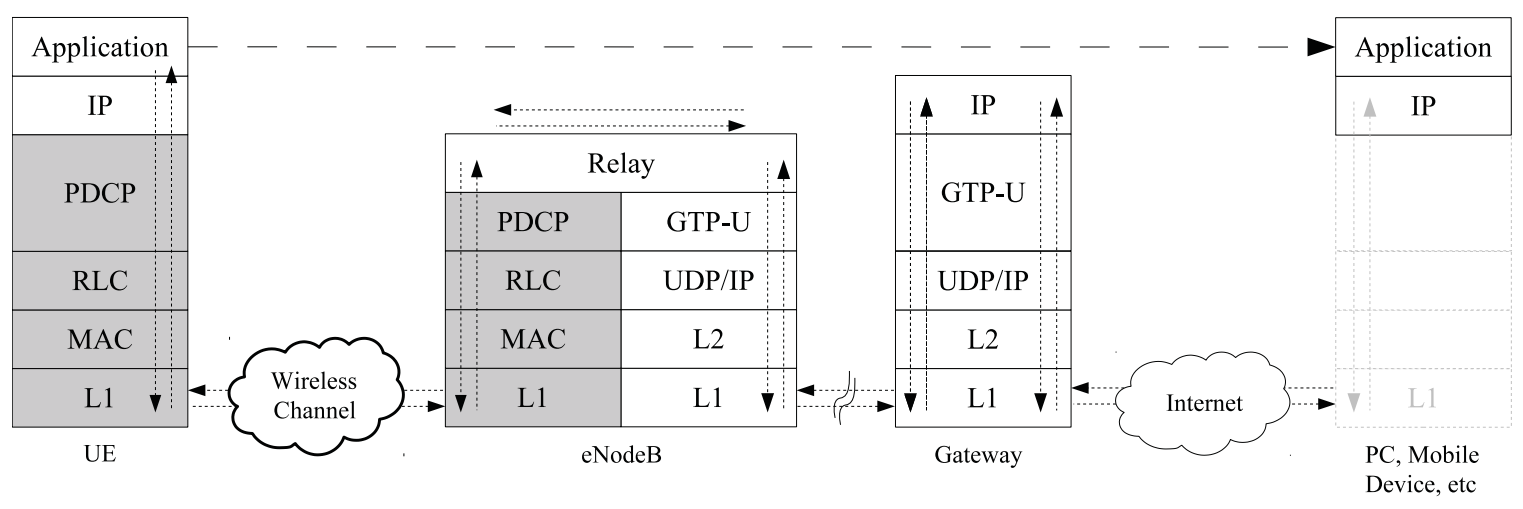

Figure 1.2: The Protocol Stack and Data Flow in an LTE Network 
Data is exchanged between the mobile station (MS) and applications running elsewhere in the network or over the Internet via the protocol stacks on each entity. To send data over the wireless channel, applications pass IP packets to the packet data convergence protocol $(P D C P)$ layer where cyphering, integrity checks and header compression are performed. The radio link control (RLC) layer provides automatic repeat request (ARQ) error detection and correction and the media access control (MAC) layer prioritizes and maps the data to the transport channels and performs error correction using hybrid automatic repeat request (HARQ). Finally, the physical layer (PHY) layer, also known as layer 1, or simply "L1" performs bit stream segmentation, modulation and coding and maps the bit streams to the frequency resources for transmission over the wireless channel.

The link between the base station and mobile device is dealt with by the PHY layer where signal processing and channel estimation attempt to estimate the channel and compensate for its effects on the transmitted symbols. From figure 1.1 it is obvious that the performance of the network is largely dependent on the wireless channel that separates the base station (BS) and the MS and the ability of the lower layers of the protocol stack (MAC and layer 1 (L1) layers) to overcome poor channel conditions in both the uplink and downlink directions. The PHY layer plays the important role of assessing the channel and using complex adaptive modulation and coding schemes to keep errors low while maximizing the amount of data that can be sent across the channel during a transmission period. In order to constantly monitor and maximize the throughput it is important to understand how the wireless channel affects the transmitted signals.

\subsection{Wireless Channels}

To understand the wireless channel it helps to separate the channel effects into several simpler components. The wireless channel is broken down into large-scale and small-scale components. The large-scale components are described by path loss and shadow fading. Path loss is the long term average received signal strength caused the loss of signal in the environment due to scattering and absorption of the electro-magnetic signal. When the mobile device moves in the environment, the path loss predicted received signal strength may vary greatly within a small area if the signal transmit from the base station is being blocked by large objects, such as buildings. The deviation from the predicted by the path loss model is known as shadow fading. 


\subsubsection{Path Loss}

Thanks to numerous researchers and many measurements, good models are available today to predict the path loss encountered by the propagating signal between the base station and mobile device in a variety of wireless environments such as wireless indoor, outdoor, urban and sub-urban settings. Path loss has been found to be closely related to the distance $d$ between the BS and MS, as seen in the free space path loss equation 1.1. Equation 1.1 also accounts for the wavelength $\lambda$ of the carrier which is related to the wireless transmission's carrier frequency by $\lambda=c / f$.

$$
P L(d B)=10 \log _{10} \frac{P_{t}}{P_{r}}=-10 \log _{10}\left[\frac{\lambda^{2}}{(4 \pi)^{2} d^{2}}\right]
$$

Popular path loss models such as the Hata or Okumara path loss models have been produced by fitting channel measurements to path loss models containing the transmitter and receiver heights as well as the distance $d$ between the BS and the MS and the carrier frequency.

\subsubsection{Fading}

The wireless channel is also modeled by fading which can be characterized as fast or slow fading. Fading refers to the rate of change of the channel in the time domain and is often modeled by Rayleigh or Ricean distributions. The signals transmit from the base station into the environment, can be thought of as travelling electro-magnetic wavefronts. The travelling wavefronts may reach the mobile device via a number of different paths and arrive at different times. The phases of the wavefronts can add constructively or destructively at the mobile device's antennas. When the signals are destructive, the received signal power can drop considerably (eg. 30 to $40 \mathrm{~dB}$ lower than the average received signal power) and the mobile station may not be able to decode the received codeword. The constructive and destructive interference varies as the mobile device moves within the environment, therefore the rate of fading is closely related to the MS velocity.

Fading channels have been studied extensively since they cause temporary outages at the MS which cause bursts of errors in digital communication systems. The early fading models described by R.H. Clarke [5] became the basis for fading channel models and simulators. Clarke's model was modified to optimize simulation time, nevertheless Clarke's model has remained a reference model in wireless since it closely models the physics of wave propagation, multiple waves arriving at the MS and the phases and amplitudes of 
these waves. The models are a necessary tool when attempting to evaluate the potential throughput, bit error rate (BER) or other characteristics of a wireless network. However, the models discussed thus far only include a single link between the single transmitter and single receiving antenna in the wireless network, known as a single input single output (SISO) channel model.

\subsubsection{Transmit and Receive Diversity: (MISO) and (SIMO) Channels}

To overcome link outages caused by large drops in signal amplitude, known as fading, between single BS and MS antenna systems, the probability of outages can be reduced by introducing additional transmit and/or receive antennas at the BS and the MS. For example, link outages can be reduced by combining the received signals at the receiver using 2 or more antennas and signal combining methods. For instance, selection combining can be used to emphasize the signal coming from the antenna with the highest received signal strength. Similarly, the probability of an outage due to fading or shadowing can be reduced significantly by adding appropriately spaced transmitting antennas, a method known as transmit diversity. In general, system performance is improved as antennas are added and any combination of transmitting and receiving antennas can be used as long as space and processing algorithms are available.

\subsubsection{Multiple-Input Multiple-Output (MIMO) Channels}

The advantages of multiple antenna systems is even greater when multiple antennas are used at both ends of the wireless system. Under the right channel conditions, more than one signal can be transmit from the BS using the same time-frequency resource (ie. without requiring more bandwidth) and the signals can be successfully decoded at the receiver. The multi-antenna system is capable of transmitting and receiving multiple codewords when the channel exhibits more than one independent path between the BS and the MS. The independent paths occur when propagating signals are affected differently by the channel and noise. This occurs when the antennas are spaced far enough apart to be uncorrelated and the propagating signals remain uncorrelated while travelling in the channel by taking different paths.

When multiple antennas are used, the links between each transmitting and receiving antenna are represented by a matrix known as the multiple input multiple output (MIMO) 
channel matrix. The MIMO channel matrix can be thought of as a number of SISO channels between each base station antenna element and each of the antenna elements at the receiver. The throughput can be greatly increased by transmitting multiple codewords. Therefore, it is necessary to properly model the channel, the antenna correlation and the antenna polarization when using MIMO channels so as not to over-estimate results.

The 3GPP has made available several correlation matrices in [6] designed to correlate the transmit and received signals at both the MS and BS. Similarly, polarization can also be accounted for using the cross-polarization discrimination ratio (XPD) in the spatial channel model. Uncorrelated signals occur when they are affected differently by noise and different channels since they travel via different paths. An uncorrelated channel may also be characterized by a large number of paths arriving at the receiver with largely varying delays, as shown in figure 1.3. The factors that affect the correlation of the signals are the angle spread of the signals departing the BS, which determines the richness of the multi-path in the channel, and the antenna spacing at the BS.

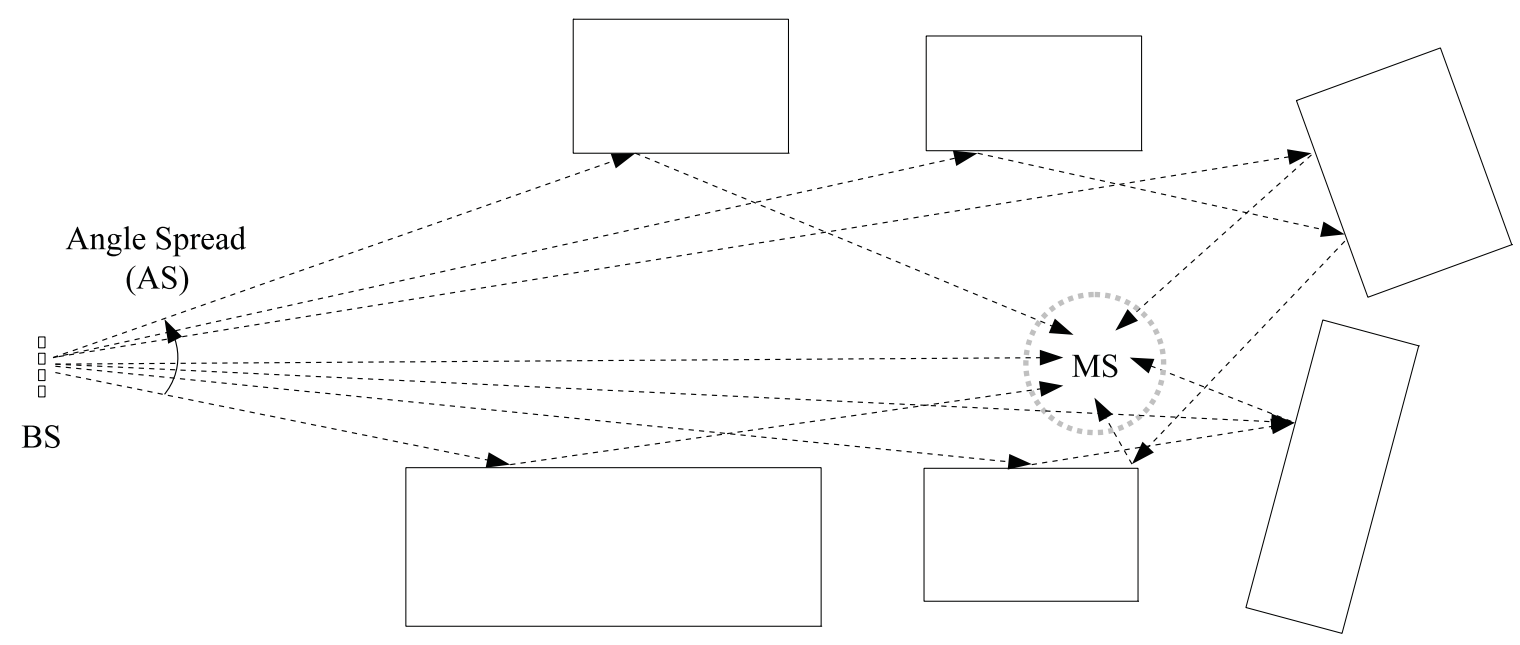

Figure 1.3: Scattering due to Angle Spread at the BS

While system designers have little control over the channel and angle spread of the signals departing the BS, the antenna correlation can be reduced by increasing the spacing of the BS antenna elements. The number of transmitting and receiving antennas and correlation between these antennas has a profound effect on the number of codewords that can be transmit simultaneously, the diversity of the transmit signals, and the efficiency and throughput of a wireless network. Advanced channel models and LTE simulators are 
needed to accurately model the LTE downlink; one such channel model is the spatial channel model.

\subsubsection{Spatial Channel Models (SCM)}

The latest channel models take modeling a step further by implementing spatial characteristics such as angles of departure and angles of arrivals for the travelling signals, better antenna field pattern models, antenna correlation and the introduction of the crosspolarization discrimination ratio (XPD). The new models have been designed to help system designers better understand the performance of new wireless systems known as beyond $3^{\text {rd }}$ generation (3G) systems, which make use of advanced concepts like multiple transmit and receive antennas to perform spatial multiplexing and beamforming. The new spatial models also improve the channel model to better match the physical characteristics of wave propagation in a wireless scenario. For example, the spatial model improves on the distribution of angles of departure of the departing signals as well as the distribution of the angles of arrival of the signals arriving at the MS.

The new multiple antenna wireless networks are opportunistic in the sense that they attempt to take advantage of the independent data streams available in the MIMO wireless channel without increasing system bandwidth or transmit power. Therefore, it is important to use channel models that adequately model the channel's spatial characteristics, otherwise, overly optimistic results may be predicted by the simulator. For this reason, this thesis will make extensive use of an advanced spatial model known as the 3GPP extended spatial channel model, or spatial channel model extension (SCME) for short, to evaluate the LTE downlink throughput under various conditions.

\subsection{Long Term Evolution}

\subsubsection{Orthogonal Frequency Division Multiplexing (OFDM)}

In the frequency domain, the transmit wireless signal occupies a defined bandwidth. The LTE air interface uses a technology known as orthogonal frequency division multiple access (OFDMA) to divide the spectrum between mobile users and the uplink and downlink. LTE splits the spectrum further into narrow sub-channels that are located at $15 \mathrm{kHz}$ intervals along the entire bandwidth of the frequency domain in order to combat fading channels. 
OFDM helps to overcome a wireless channel impairment known as frequency selective fading. In the frequency domain, frequency selective fading is the rapid variation of the channel amplitude across the system bandwidth. The rapid amplitude fluctuations in the frequency domain are caused by many delayed multi-path components. As the arriving multi-path delays increase, the channel becomes more frequency selective. To compensate for the channel amplitude variation the channel is sampled at particular points in the frequency domain at the location of known signals, called reference signals (RSs). The RSs are used to estimate the amplitude of the channel across the frequency domain and remove the channel's effect. The technique is known as frequency domain equilization (FDE). In addition to FDE, the RSs are also used to determine and report the channel quality for future transmission.

The system control loop that assesses the channel quality at particular OFDM subcarriers, then adjusts the transmission scheme to match the channel is known as link adaptation. Link adaptation is a topic of interest in wireless OFDM systems due to the required fine tuning and impairments that the link adaptation control system may be subject to and its importance to the optimal performance of the LTE downlink.

\subsubsection{Link Adaptation}

The quality of the link between the BS and user equipment (UE) plays an important role in the quality of service and throughput that can be achieved. Therefore, understanding the wireless channel and wireless propagation is a very important step in the design of a high performance network. The capability to overcome poor channel conditions and system impairments with link adaptation is imperative to maintain a standard of service and throughput while working within a fixed bandwidth allocation.

Significant research on wireless channels and measurements have led to the complex channel models that exist today. Using the available models has enabled a faster, less costly and more accurate research and development cycle. The implementation of low complexity and high complexity models at the link level and system level have led to link adaptation algorithm performance comparisons and system design choices that would otherwise require mock-up networks to be built and drive tests to be conducted. Since LTE is an opportunistic technology which relies heavily on link adaptation, link adaptation will be an important part of this thesis and the LTE simulations all incorporate link adaptation using various adaptation complexities. 


\subsection{Thesis Motivation}

In the wireless industry it is well known that demand for bandwidth is increasing rapidly and the ability to always be connected while being mobile is far more attractive to consumers than being tethered by wires. Spectrum auctions are increasingly competitive and expensive and spectrum is limited in nature. Globally, government organizations are looking to aggregate partitioned spectrum, relocate services to make new spectrum available and reuse spectrum where possible by permitting agile technologies, such as cognitive radio, to use already allocated spectrum. Making better use of the available spectrum through new technology enables lower cost services to be deployed with higher bandwidths. In the era of commercial wireless MIMO technology, operators are able to get higher spectral efficiencies within the same bandwidth than ever before by using multiple transmitters and receivers. The work in this thesis is motivated by the need to improve throughput and spectral efficiencies for the benefit of consumers and wireless companies by evaluating LTE under a number of multi-antenna configurations, along with impairments in order to improve the throughput and spectral efficiency of the LTE downlink.

\subsection{Objectives}

In this thesis the channel and impairments will be discussed from the point of view of the LTE downlink in order to evaluate the downlink performance. The thesis will need to cover the wireless channel, LTE downlink transmission modes and the link adaption schemes that allows LTE to maximize the use of the available spectral resources. Like any other feedback based control system, the reporting of feedback in LTE for link adaption is not always ideal due to system trade-offs and this can lead to reduced performance. The LTE antenna configurations and LTE parameters that can be adjusted to improve the downlink performance will be varied to show changes in performance. Based on the results, the best antenna configurations and LTE parameters will be suggested.

Three topics have been selected to evaluate the downlink of a four transmitter by two receiver LTE system, compared to two by two LTE system. The simulations within each topic are used to compare the performance of the LTE downlink while subjecting the downlink transmissions to the following impairments and configurations: 
- Phase jitter

- Channel variations due to UE mobility

- Unreliable channel state information

- Antenna correlation

- Antenna polarization

The studies presented in chapter 4 will focus on a particular topic or impairment but the underlying focus is the performance of the 4 transmitter package at the base station which is expected to help boost the throughput and improve spectral efficiencies of LTE networks for wireless carriers compared to the less complex 2 transmitter antennas, currently in use.

The objectives of this thesis are to answer the following questions:

- What is the effect of various antenna configurations on the downlink throughput and what are the optimal configurations?

- What is the effect of real phase jitter between antenna ports on the throughput performance of the LTE downlink?

- What is the effect of velocity and non-ideal channel state information on the throughput performance of the downlink?

\subsection{Thesis Contributions}

This thesis contributes by presenting simulation results and analysis which helps to better understand the areas discussed in the objectives. The thesis contributions are:

1. A complete overview of LTE, link adaptation, transmission schemes, wireless channels and state of the art spatial channel modeling for LTE link level simulations is presented.

2. An analysis of the throughput performance of a number of antenna configurations is presented and the best antenna configurations and transmission modes are suggested based on the scenario.

3. Generated and real system phase jitter is introduced into the LTE system and the effect on the downlink throughput is discussed.

4. Finally, the effect of non-ideal channel state information (CSI) and UE velocity at vehicular and high speed train speeds on the downlink are discussed based on a number 
of SCME simulations.

Students will benefit from this thesis by gaining an understanding of a modern wireless communication system and the impairments that must be overcome to design a well performing wireless link layer.

A detailed presentation of the wireless channel and downlink transmission schemes (transmission mode 2 (TM2), transmission mode 3 (TM3), transmission mode 4 (TM4) and transmission mode 6 (TM6)) will be given, along with performance figures for many transmission modes coupled with various antenna configurations (low correlation, high correlation cross-polarized and co-polarized linear arrays). The impact of impairments (phase jitter, non-ideal channel state information (CSI) feedback reporting rates, Doppler) will also be treated by simulation work and detailed discussions.

Researchers and systems designers will be able to benefit from this work by making better decisions about their networks based on the expected environment and other conditions. Furthermore, researchers can gain some insight into current system designs and some of their weaknesses which may persuade them to develop innovative improvements.

\subsection{Organization of the Thesis}

The thesis is organized to introduce the reader to basic theory behind wireless fading channels in section 2.1 and OFDM technology in section 2.2. SISO channels, MIMO channels and wave propagation and the received electric field will be given in sub-sections 2.3.2, 2.3.3 and 2.3.4.

Before venturing into the details of the advanced spatial channel model, the reader is introduced to the popular sum of sinusoids (SoS) physical model developed by R.H. Clarke, in section 2.3.5, and improvements made to this model by Jakes, in section 2.3.6. Due to the need for an advanced simulator to simulate and obtain performance results for the spatial multiplexing transmission modes of interest in this thesis, the spatial channel model is presented. Spatial channel modeling is discussed in detail in sub-section 2.3.7 where the focus is placed on the extended spatial channel model (SCME) adopted by the 3GPP.

In section 2.4, the LTE layer 1 air interface is discussed and basic parameters defining LTE's OFDM transmission scheme are presented. An overview of the OFDM transmitter chain is given in sub-section 2.4.1 where important coding, modulation, resource mapping and OFDM symbol generation take place before transmission. 
Once the reader has acquired the necessary knowledge of LTE transmissions and the wireless channel, channel estimation, link adaptation and transmission modes are presented in section 2.4.5, 2.4.6 and 2.5. The LTE transmission modes of interests in this thesis (transmit diversity TM2, open-loop spatial multiplexing TM3 and closed-loop spatial multiplexing TM4), will be discussed in detail in sections 2.5.2, 2.5.3 and 2.5.4.

In chapter 4 , three studies are presented corresponding to the questions asked in the objectives in section 1.6. The first study in sub-section 4.1 discusses antenna configuration simulations. The second study will present simulations of a radio frequency (RF) impairment. Real lab measured phase jitter will be used in LTE simulations to simulate phase noise and the results will be discussed in section 4.2. Finally, the third performance evaluation in sub-section 4.3 looks at the throughput performance of a LTE system with increasing UE velocities along with ideal and aged channel quality indicator (CQI) values at three CQI reporting intervals. Each study contains a short description of the purpose of the study relating to the objective in sub-section 1.6. In addition, the studies will give a brief introduction of the simulation scenario and parameters used to run the simulations and then a discussion of the results will follow.

Finally, chapter 4 contains a brief summary and conclusion of the results and work completed in this thesis, in sub-section 5.1. And last but not least, a discussion covering possible future work concludes this thesis in section 5.2. 


\section{Chapter 2}

\section{Background}

In this chapter, the reader will be introduced to the wireless channel and the extended spatial channel model (SCME) used in the simulations in chapter 3. Once versed in the theory of the wireless channel models, section 2.4 will present the reader with the details of the LTE physical layer and the key components, such as frequency division multiple access (FDMA), link adaptation, frequency selective scheduling and transmission modes like spatial multiplexing that make the LTE physical layer an efficient wireless technology.

\subsection{Wireless Fading Channels}

The performance of the mobile system is dependent on the radio channel and its ability to overcome the effects of the radio channel. The channel is typically broken into path loss, shadow fading and multi-path propagation. Path loss and shadow fading affect the average received signal strength and are discussed in section 2.1.1, while multi-path propagation and fading are explained in section 2.1.2.

\subsubsection{Path Loss and Shadowing}

Path loss is the loss of signal strength caused by the signal dispersion in the environment. The path loss component of a wireless channel model is a function of the distance between the base station (BS) and the receiver. Typical path loss models also include large-scale shadowing in the path loss equation to account for the effects of obstructions, such as large buildings or destructive interference. A log-normal variable with zero mean and typical standard deviation of $\sigma=8$ is used to model the effect of large-scale shadowing.

A simple path loss equation taken from [7] is shown in equation 2.1. The path loss 
model in equation 2.1 accounts for the distance $d$ between the mobile device and the base station and large-scale shadowing $X_{\sigma}$. The model also includes the free space path loss exponent $n$, which determines the power decrease with distance $d$, and the reference distance $d_{0}$.

$$
P L d B(d)=\bar{P} L(d)+X_{\sigma}=P L_{f}\left(d_{0}\right)+10 n \log \left(\frac{d}{d_{0}}\right)+X_{\sigma}
$$

Path loss models have been modified and fitted to field data in order to model various scenarios. A popular model, and one used in this thesis, is the COST-231 Hata urban path loss model shown in equation 2.2. The model is specified in [3] for the urban macrocell and suburban macrocell environments.

$$
\begin{aligned}
P L d B(d)= & \left(44.9-6.55 \log _{10}\left(h_{b s}\right)\right) \log _{10}\left(\frac{d}{1000}\right)+45.5 \\
& +\left(35.46-1.1 h_{m s}\right) \log _{10}\left(f_{c}\right)-13.82 \log _{10}\left(h_{b s}\right)+0.7 h_{m s}+C
\end{aligned}
$$

Path loss equation 2.2 is a function of the MS-BS distance $d$. The model's inputs also include the base station height $h_{b s}$ in meters, the mobile station height $h_{m s}$ in meters, the carrier frequency $f_{c}$ in $\mathrm{MHz}$ and the constant $\mathrm{C}$, where $\mathrm{C}$ is $0 \mathrm{~dB}$ for suburban of $3 \mathrm{~dB}$ for urban scenarios.

The path loss model shown in equation 2.2 is the model used in the spatial channel model that is later described and then used in chapter 4 .

\subsubsection{Multi-Path Propagation and Small-Scale Fading}

Multi-path propagation occurs when multiple components of the transmit signal propagate through the environment due to scattering and arrive at the receiver at different times and via different paths. The result of signal scattering and signals reflecting off of large objects causes the multi-path components (MPCs) to travel different distances before arriving at the receiver, as seen in figure 2.1. The MPCs arriving at the receiver can be represented by a power-delay profile, also known as a channel impulse response as shown in figure 2.2.

In order to model the wireless channel the channel impulse response (CIR) is used to represent the multi-path components arriving at the receiver. The channel impulse response $h(t)$ represents a snapshot of the channel multi-path components at a particular instant in 


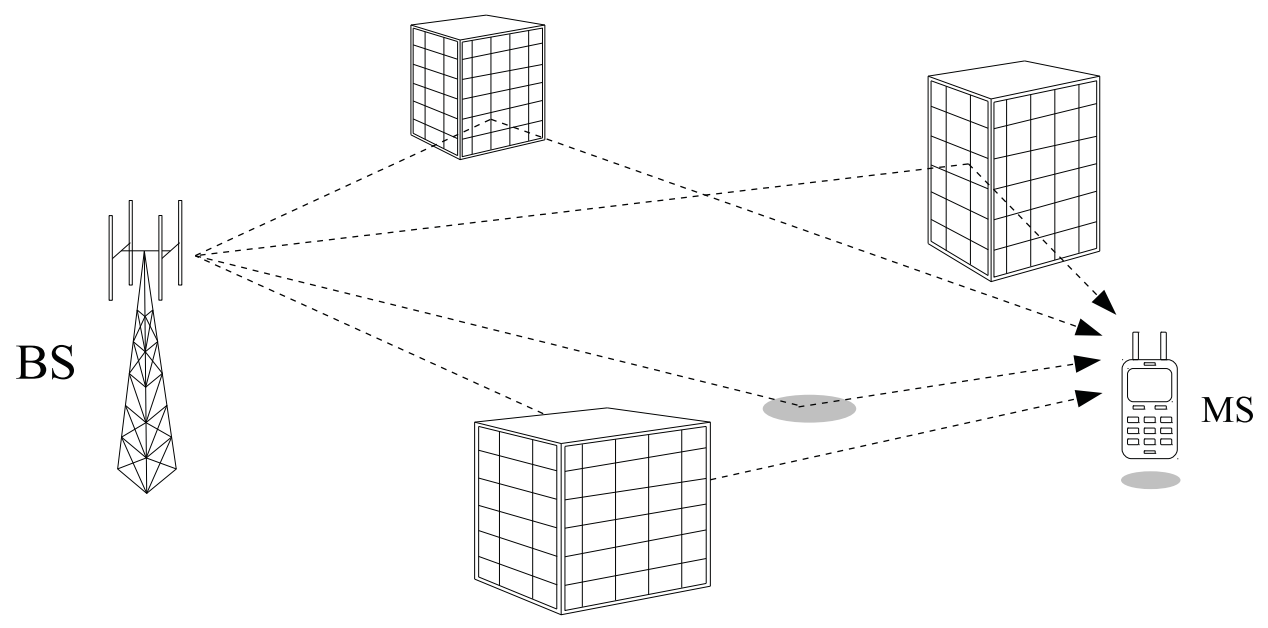

Figure 2.1: Multi-Path Propagation

time. Each tap in the channel impulse response represents the amplitude and phase of the multi-path arriving at a particular point in time. The amplitude of $h(t)$ can take various shapes but it is often approximated using an exponentially decaying model.

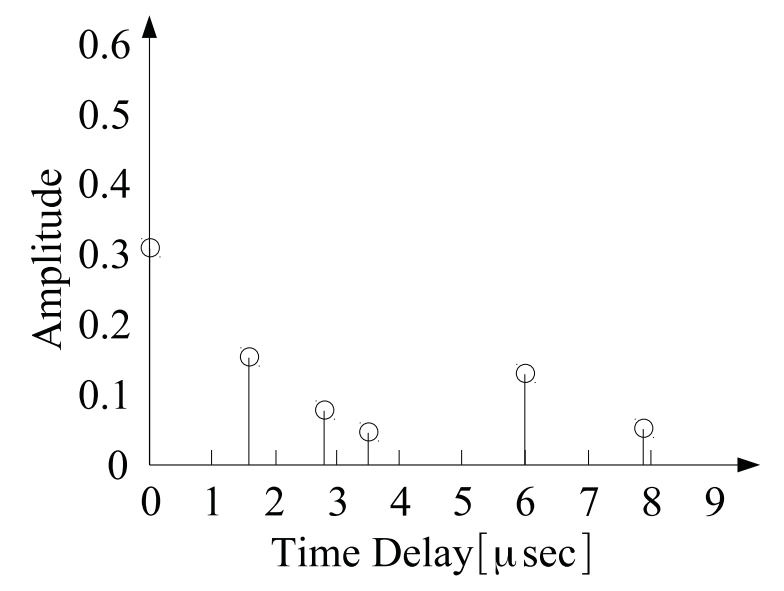

Figure 2.2: Channel Impulse Response $h(t, \tau)$ at time $t$

The delay of the first multi-path component to arrive at the receiver is known as the propagation delay. The channel impulse response is typically normalized by subtracting the delay of the first component from all the delays of the subsequently arriving signals. The delay of the first and usually strongest multi-path component is the propagation delay and can be approximated by $\tau=d / c$ especially if a line-of-sight (LOS) component exists, where $\tau$ is the propagation delay, $d$ is the BS-MS distance and $c$ is the speed of light in free 
space.

\section{RMS Delay Spread}

Useful information can be derived from the characteristics of the channel impulse response like the root mean square (RMS) delay spread $\sigma_{\tau}$ of the channel. The RMS delay spread $\sigma_{\tau}$ is calculated using equation 2.3 using the mean delay from equation 2.4 and the mean square delay from equation 2.5 all from [7].

$$
\begin{gathered}
\sigma_{\tau}=\sqrt{\overline{\tau^{2}}-(\bar{\tau})^{2}} \\
\bar{\tau}=\frac{\sum_{i} a_{i}^{2} \tau_{i}}{\sum_{i} a_{i}^{2}} \\
\overline{\tau^{2}}=\frac{\sum_{i} a_{i}^{2} \tau_{i}^{2}}{\sum_{i} a_{i}^{2}}
\end{gathered}
$$

The RMS delay spread can help to determine the type of fading and also help make design choices that lead to a well functioning LTE OFDM wireless system.

\section{Small-Scale Fading}

The channel changes over time if the receiver or large objects in the environment are in motion, therefore a real wireless channel is time-variant. As the receiver or other large objects in the environment move, the signals received at the MS add constructively and destructively due to the differences in their phases. In figure 2.3, the time varying channel envelope can be seen to change according to the well known Rayleigh distribution which is used to model wireless channels.

The variations in the channel envelope is known as small-scale fading. In a typical wireless radio access network (RAN) the base station is fixed therefore the movement is considered to be due to the MS moving within the cell. The fading envelope in figure 2.3 shows the slowly and rapidly changing variations in the channel, known as slow fading and fast fading and is due to three aspects which will be discussed further in section 2.1.2.

- Multi-path propagation

- Speed of the MS

- Changes in the environment due to large objects (eg. vehicules) and the speed at which these changes occur. 


\section{Slow and Fast Fading}

The rate of the channel's fast fading is related to the rate of change of the MPCs of the channel impulse response (CIR) at the receiver due to the above three environmental and MS factors. Fading is categorized as slow fading or fast fading. Slow fading occurs when the channel coherence time $T_{c}$, given by equation 2.6, is longer than the symbol transmission time $T_{s}$. This implies that the signal does not change during the transmission of a symbol. The bandwidths of the baseband signal $B_{s}$ and Doppler spread $B_{D}$ can also be used to categorize slow fading. Slow fading is occurs if the condition $B_{s}>>B_{D}$ is met.

$$
T_{c} \approx 1 / f_{m}
$$

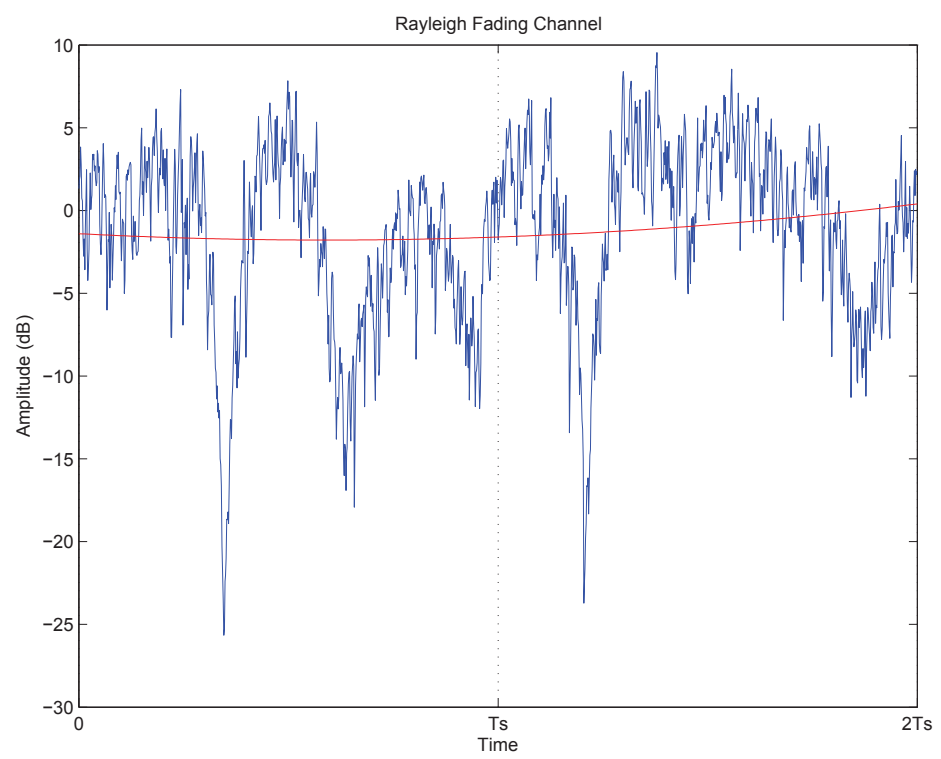

Figure 2.3: Slow and Fast Rayleigh Fading

On the other hand, a fading channel is considered to be exhibiting fast fading if the condition in 2.7 is met. Fast fading occurs when the channel coherence bandwidth $T_{c}$ is less than the symbol period $T_{s}$, implying that the channel changes within a symbol transmission period. The inverse of the channel coherence time $T_{c}$ and the signal period $T_{s}$ give a similar 
condition for fast fading using the baseband signal bandwidth $B_{s}$ and the Doppler spread $B_{D}$ as shown in 2.8 .

$$
\begin{aligned}
& T_{s}>T_{c} \\
& B_{s}<B_{D}
\end{aligned}
$$

\subsubsection{Frequency Flat and Frequency Selective Channels}

The MPCs arriving at the receiver with different delays can cause the channel to become frequency selective as opposed to frequency flat. The frequency selectivity of a channel can be observed by obtaining the frequency domain representation of the channel using the channel impulse response and the well known Fourier transform, shown in equation 2.9.

$$
H(t, f)=\mathcal{F}\{h(t, \tau)\}
$$

The delay spread $\sigma_{\tau}$, calculated using equations $2.3,2.4$ and 2.5, is useful in determining the frequency selectivity of the channel.

\section{Frequency Flat Fading}

A frequency flat channel is characterized as a channel with a relatively constant amplitude across the signal bandwidth as shown in figure 2.4. Specifically, frequency flat channels are channels where the coherence bandwidth $B_{C}$ of the channel is larger then the bandwidth of the signal $B_{s}$, thus satisfying condition 2.10 .

$$
B_{C}>>B_{s}
$$

In the time domain, a channel is classified as frequency flat if the symbol period $T_{s}$ is greater than the delay spread $\sigma_{\tau}$ of the channel impulse response, satisfying condition 2.11.

$$
T_{s}>>\sigma_{\tau}
$$

Frequency flat channels are desirable since equalization at the receiver is much simpler. When the channel is assumed flat over the signal bandwidth $B_{s}$, only a simple single tap frequency domain equalizer is required to counter the multiplicative channel effect for the 


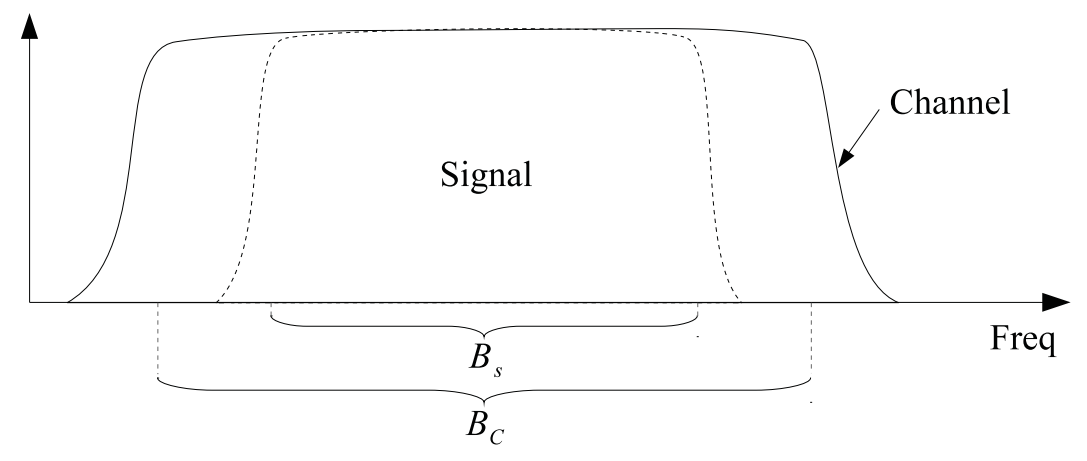

Figure 2.4: Frequency Flat Channel

entire signal bandwidth $B_{s}$. To counter channels with narrow coherence bandwidths and maintain low complexity at the receiver, the transmission bandwidth is divided into subchannels in order to maintain the frequency flat channel property across the individual sub-channels.

\section{Frequency Selective Fading}

Frequency selectivity is caused by a channel which exhibits multiple significant taps (multipath components) in the channel impulse response. The Fourier transform of the channel impulse response of a frequency selective channel will have a changing amplitude and phase across the frequency domain due to the multiple paths taken by the propagating signals, as seen in figure 2.5. Channels are categorized as frequency selective when the bandwidth of the baseband signal $B_{s}$ is larger than the coherence bandwidth $B_{C}$, satisfying the condition in 2.12 .

$$
B_{s}>B_{C}
$$

In the time domain, a channel that satisfies condition 2.13 leads to categorizing the channel as frequency selective. The condition 2.13 is simply the time domain version of the frequency domain condition in 2.12 , described earlier.

$$
T_{s}<\sigma_{\tau}
$$

A frequency selective channel can have large drops in signal amplitude in the frequency 


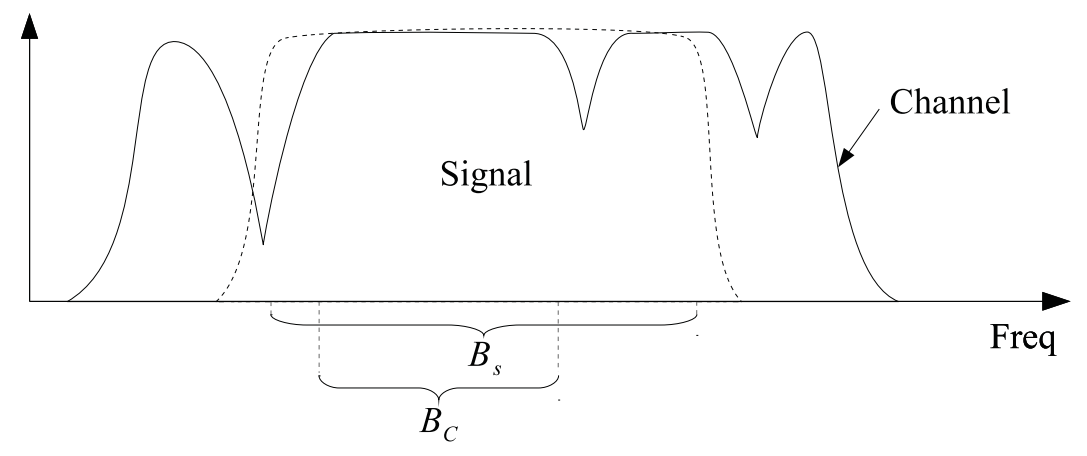

Figure 2.5: Frequency Selective Channel

domain due to significant and delayed multi-path components visible in the channel impulse response. The receiver must compensate for the frequency selective nature of the channel using channel equalization techniques. Equalization is the process of estimating the channel and applying the inverse of the channel, known as the matched filter, to remove the channel effects.

In LTE, equalization is accomplished in the frequency domain. Channel estimation and equalization are accomplished by inserting known reference signals in the frequency domain to estimate the channel, as discussed in section 2.4.4. Using the known reference signals the channel can be estimated and the channel effect can then be removed, as discussed in section 2.4.5

In the case where a wide signal bandwidth is desired for high bit rates and the channel is highly frequency selective, frequency selectivity is overcome by splitting the bandwidth of a communication system into several narrow band and frequency flat sub-channels. If the frequency domain spacing of the sub-channels (commonly known as sub-carriers) is selected to be smaller than the channel's coherence bandwidth $B_{C}$, then the a simple single tap equalizer (single complex multiply) can be used to equalize each sub-carrier.

Narrow-band sub-carrier design used to split a wide bandwidth into many parallel subchannels is known as frequency division multiplexing (FDM) and will be introduced in section 2.2. A efficient form of FDM, known as OFDM, and frequency domain equalization are utilized in modern communication systems like LTE to overcome the frequency selective channels that are common in wireless environments. The careful selection of the system parameters allows the wireless system to counter channel effects present in the wireless channel. 


\subsection{Orthogonal Frequency Division Multiplexing (OFDM) Systems}

OFDM is a widely used multi-carrier communication scheme. Multi-carrier communication schemes divide the allocated spectrum into several sub-channels that individually carry data in parallel sub-channels, as shown in figure 2.6. This is equivalent to dividing a wide bandwidth signal, carrying information at high bit rates, into many parallel narrowbandwidth sub-channels collectively carrying the same high bit rate data stream. This section introduces OFDM, which is a popular, efficient and flexible multi-carrier transmission scheme used by LTE and other modern wireless technologies.

\subsubsection{OFDM}

Orthogonal frequency division multiplexing (OFDM) is an efficient form of the multicarrier scheme known as frequency division duplexing (FDM). The OFDM sub-carriers, shown in figure 2.6, divide the spectrum into several parallel narrow-band sub-carriers that are designed to be frequency flat under typical conditions. The narrow-band frequency flat sub-carriers of an OFDM system are grouped together to allow high bit rate communication systems to be developed while keeping the complexity of the receiver's equalizer low.

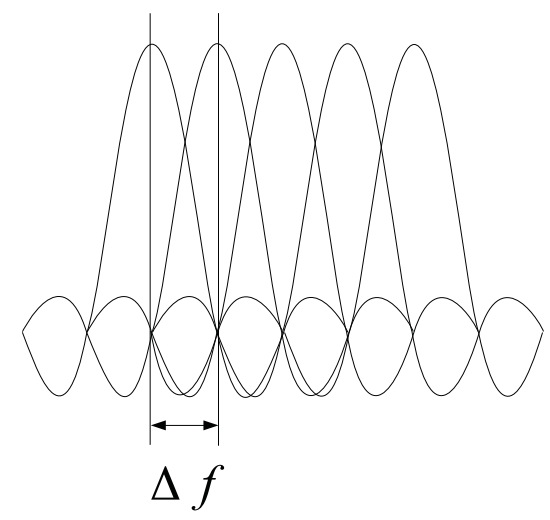

Figure 2.6: OFDM Sub-Carriers with Frequency Domain Spacing $\Delta f$

An OFDM communication system converts complex modulated symbols allocated to sub-carriers in the frequency domain into time domain samples just before up-conversion 
and transmission using the inverse fast Fourier transform (IFFT). After the time domain samples have been transmit over the wireless channel, the received signal is downconverted at the receiver and time domain samples are converted to modulated frequency domain symbols using the fast fourier transform (FFT) to recover the modulated symbols. The receiver extracts the modulated symbols from the transmitted signal by sampling the sub-carriers at the peak of each sub-carriers where the interference from the adjacent subcarriers is lowest.

In LTE, the sub-channel bandwidth $B_{s}$ (or frequency domain spacing $\Delta f$ ) is selected to be smaller than the typical coherence bandwidth $B_{c}$ observed in urban and sub-urban environments in order to ensure the sub-carriers experience frequency flat fading. When the narrow-band sub-carriers can be assumed frequency flat, channel equalization can be accomplished with a simple complex multiplication per sub-carrier.

When designing OFDM systems, the symbol period $T_{s}$ must also be selected with great care. The symbol period is chosen to be longer than the delay spread of the channel $\left(T_{s}>>\right.$ $\sigma_{\tau}$ ) in order to reduce the effect of inter-symbol interference (ISI). A longer symbol period implies that a smaller portion of the time domain samples of the OFDM symbol will be corrupted by the previous symbol transmission. The cyclic prefix, discussed in section 2.2.2, is also employed to reduce the ISI caused by the dispersive channel.

\subsubsection{Cyclic Prefix (CP)}

The cyclic prefix (CP) is a critical part of an OFDM communication system because it is used to remove inter-symbol interference (ISI) caused by a dispersive or band-limited channel. When a time domain OFDM symbol is transmit from the BS, copies of the symbol arrive at the receiver via different paths. The delayed copies of the symbol cause overlap between the transmit symbols. The tail end of the delayed versions of symbol $T_{0}$ arrive at the receiver as the beginning of the next OFDM symbol, symbol $T_{1}$, is received. The overlap, shown in figure 2.7, is known as ISI and can be removed using the cyclic prefix and careful system design.

The CP is similar to a guard interval, however, the redundant information transmit in the time domain samples corresponding to the $\mathrm{CP}$ also has other important uses. The $\mathrm{CP}$ is formed at the BS just before transmission by making a copy of the end of the original time domain OFDM symbol and then pre-pending the copied samples to the front of the original OFDM symbol. The ISI caused by the previously transmitted symbols can be removed if 


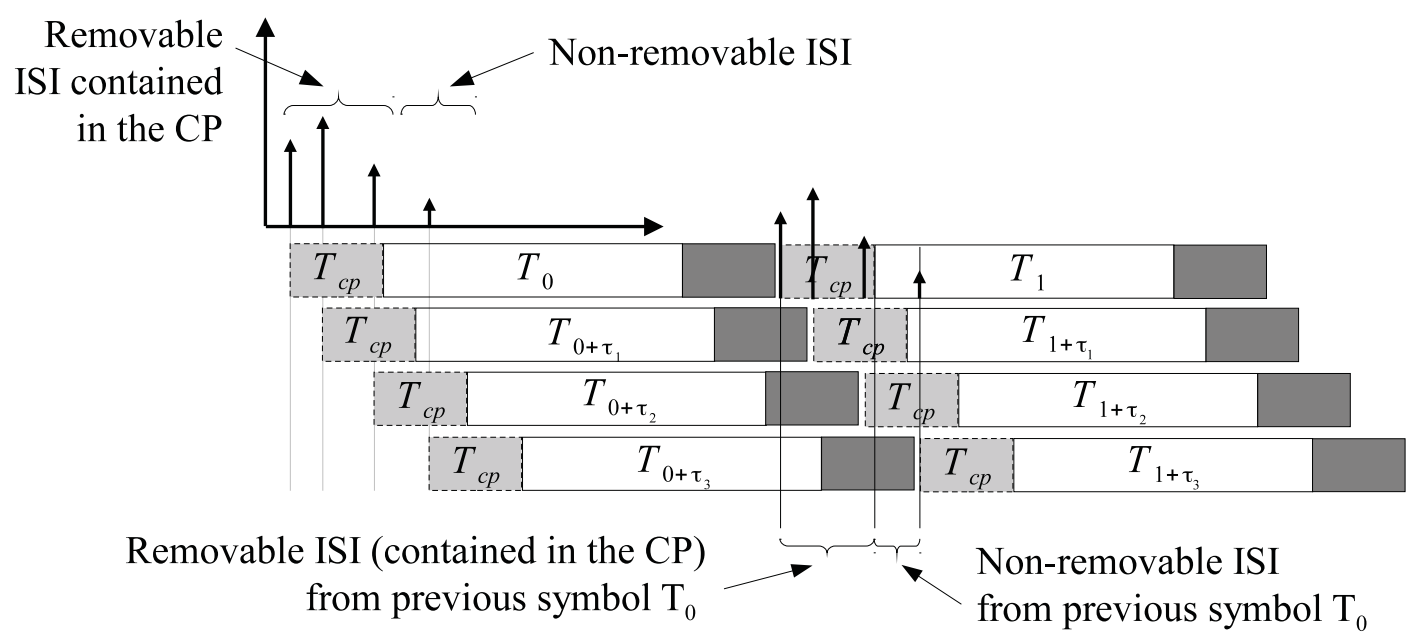

Figure 2.7: OFDM Cyclic Prefix with Inter-Symbol Interference

it falls within the cyclic prefix by discarding the corrupt time domain samples of the $\mathrm{CP}$ at the receiver before performing the FFT.

The CP reduces the data rate of the OFDM system, however, it does serve another important function in addition to removing ISI. The $\mathrm{CP}$ changes the effect of the channel from a linear convolution to a perceived circular convolution. The benefit is that the received signal after discrete Fourier transform (DFT) can be viewed as $X_{k} \cdot H_{k}$, where $X_{k}$ is the modulated frequency domain symbol at sub-carrier $k$ and $H_{k}$ is the channel experienced by that sub-carrier.

\subsection{Channel Modeling}

Mobile channel models are of out most importance to the wireless systems engineer. Channel models allow system designers to reproduce results in a controlled environment and to vary only the system parameters that are of interest. For instance, the ability to have precise control over channel parameters allows a researcher to verify the system's performance under various scenarios. Wireless channel models can also be used to identify opportunities that may exist and design a more efficient communication system that takes advantage of opportunities provided by the channel by link adaptation, scheduling or the transmission of multiple codewords when possible.

The following section introduces MIMO channel modeling, the received electric field and channel models that have been used as the building blocks for the more complex spatial 
channel models that exist today. The discussion begins with the motivation for channel models, followed by the MIMO channel matrix representation, the received electric field and Clarke's sum-of-sinusoids (SoS) physical model.

The sum-of-sinusoids (SoS) method used in Clarke's model is the basis for the state of the art 3GPP spatial channel model 3GPP/3GPP2 spatial channel model (SCM) and extended spatial channel model SCME used extensively today. Spatial channel models are required to obtain accurate simulation results in LTE due to the use of beamforming and advanced transmission modes like open-loop spatial multiplexing (OLSM) and closed-loop spatial multiplexing (CLSM) that make use of the channel's spatial characteristics. The spatial channel model, introduced at the end of this section, is better equipped to model the physical and spatial characteristics of the antennas and channel at the wide bandwidths used in communication systems today.

\subsubsection{Motivation for Channel Models}

Standardized channel models provide a repeatable test environment which allows researchers to compare the performance of their algorithms to other researcher's results. Without standard models, results could not be reproduced accurately and it would be impossible to select the best algorithms available. Channel modeling has lead to precise comparisons of new ideas leading to more efficient systems such as the systems that are being deployed today.

The creation of channel models relies on our ability to develop an understanding for the physical environment that wireless signals propagate within. The simple act of understanding the wireless environment in order to define a model, is the first step toward improving the current models in use today.

Finally, companies are motivated to develop simulators based on channel models since they allow for a more rapid research and development cycle. Models reduce cost and time since simulations can be quickly configured and algorithms can be added and tested by many engineers without the need to setup expensive hardware and find spectrum for drive testing for each project.

\subsubsection{Single Input Single Output (SISO) Wireless Channel}

A basic wireless communication link between a single transmitter and single receiving antenna is represented by a channel with a single link and single channel impulse response. 
A channel with a single link and single channel impulse response is known as a single-input single-output (SISO) channel.

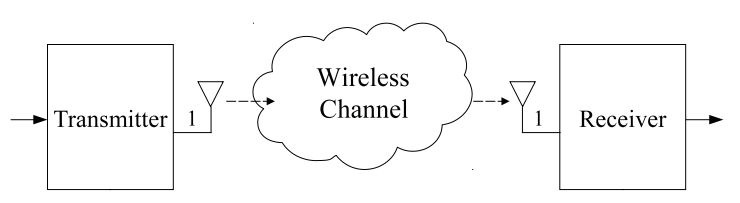

(a) SISO Channel

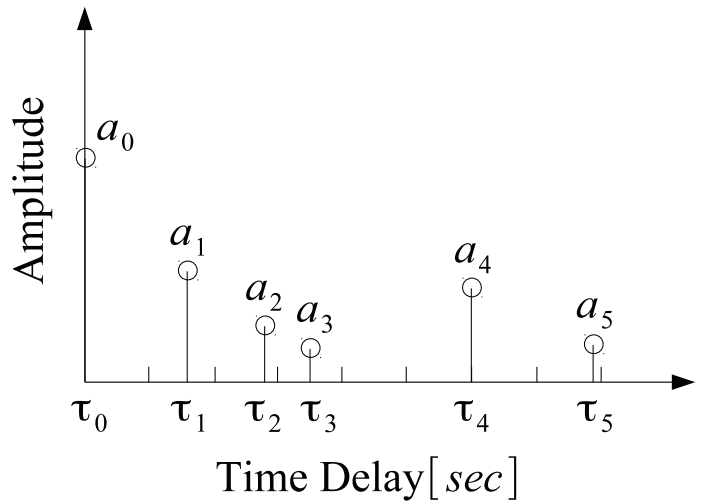

(b) Channel Impulse Response for the SISO Channel

Figure 2.8: SISO Channel Model and Channel Impulse Response

The propagating signal can be represented by one or more rays travelling in the environment. The ray that travels to the receiver via the shortest direct path is known as the LOS ray and is typically the strongest signal received at the receiver. Scattering in the channel causes other rays to travel longer distances by reflecting off of large objects before arriving at the receiver. The signals propagating in the environment arrive at the receiver at different times due to the varying lengths of the paths that each propagating signals follows. The multi-path received at the mobile station's antenna is represented by a single tap with amplitude $a_{i}$ and delay $\tau_{i}$ in the channel impulse response shown in figure $2.8 \mathrm{~b}$.

When the signal $x(t)$ is transmit from the BS antenna the received signal is represented by $y(t)$ in equation 2.14 from [8]. The received signal $y(t)$ is found by convolving the channel coefficients, represented by amplitudes $C_{i}$ and phases $\phi_{i}$ at time $t$, and the transmit signal $x(t)$.

$$
y(t)=\sum_{i=1}^{L} C_{i}(t) e^{-j \phi_{i}(t)} x\left(t-\tau_{i}\right)+n(t)
$$

\subsubsection{Multi-Input Multi-Output (MIMO) Wireless Channels}

In modern systems such as LTE, the number of transmit and receive antennas can be greater than one. When the base station and mobile device have more than one antenna element, 
the channels between the individual antenna elements needs to be modeled. To properly model the signals transmit and received by all antennas, the MIMO channel model is used as shown in figure 2.9.

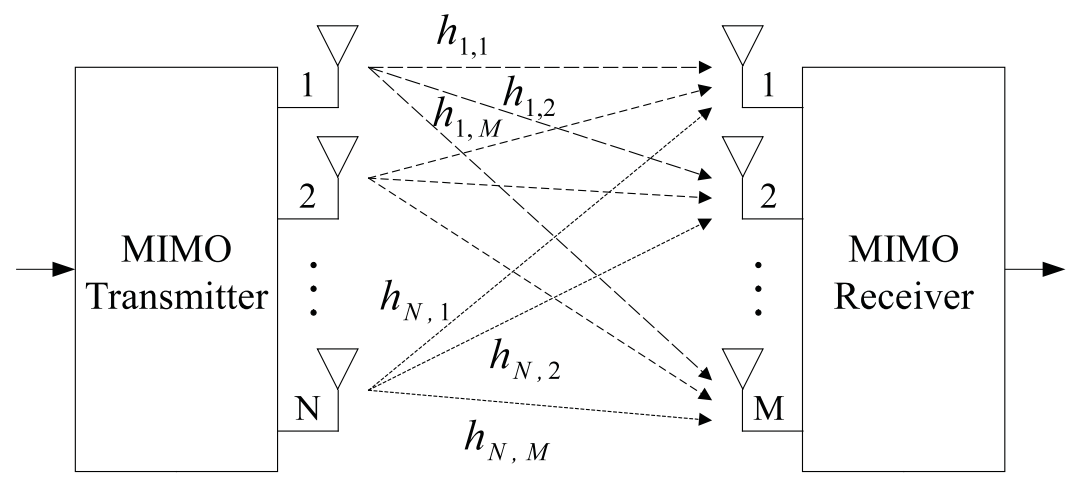

Figure 2.9: MIMO Channel

A typical implementation of a MIMO channel can be based on generating multiple SISO channel realizations between each transmitter and each receiving antenna. Each SISO channel within the MIMO channel matrix represents a link between a transmitter and a receiver antenna element and is characterized by its own unique channel impulse response (CIR) in the time domain, as shown in figure 2.10.

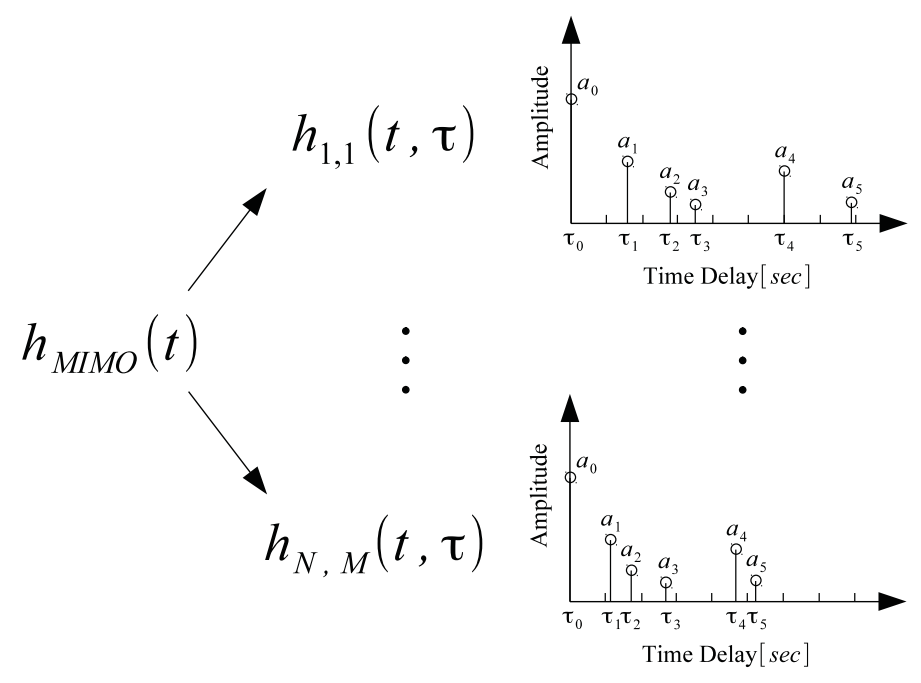

Figure 2.10: MIMO Channel Impulse Responses

The MIMO channel matrix $H_{M I M O}(t)$ is of size $N$ by $M$, representing the $N \cdot M$ transmitter-receiver links. The transmit signal is subject to the $N \cdot M$ complex channel 
gains between the transmitters and the receivers as show in 2.15 .

$$
H_{M I M O}=\left[\begin{array}{cccc}
h_{1,1} & h_{1,2} & \ldots & h_{1, N} \\
h_{2,1} & h_{2,2} & \ldots & h_{2, N} \\
\vdots & \vdots & \ddots & \vdots \\
h_{M, 1} & h_{M, 2} & \ldots & h_{M, N}
\end{array}\right]
$$

At the mobile device, the samples received after transmission through a MIMO channel can be represented by a column vector $\vec{y}$ of size $M x 1$. The symbol $\vec{y}$ is related to the transmit symbol $\vec{x}$ by equation 2.16 , where the transmit symbol is also a column vector but of size $N x 1$. The effect of the channel and noise are modeled by the $M x N$ MIMO channel matrix $\boldsymbol{H}$, which contains the channel gains between all the transmitter and receiver elements, and the $M x 1$ complex circular symmetric additive white Gaussian noise (AWGN) noise vector $\vec{w}$.

$$
\vec{y}=\boldsymbol{H} \vec{x}+\vec{w}
$$

An important property of the MIMO channel matrix is its rank. The rank of the MIMO is a measure of the capacity of the channel matrix since it reveals the number of independent data streams present in the channel. The rank of $H_{M I M O}$ can be found by singular value singular value decomposition (SVD) or eigendecomposition. SVD or eigendecomposition finds the non-zero singular values, labeled $\lambda_{1}, \lambda_{2} \ldots \lambda_{n_{\text {min }}}$, or non-zero eigenvalues, labeled as $\lambda_{1}^{2}, \lambda_{2}^{2} \ldots \lambda_{n_{\text {min }}}^{2}$, that are present in the channel matrix. The singular values or eigenvalues can be viewed as parallel channels present in the MIMO channel and are commonly known as data streams.

In other words, the SVD is used to convert the MIMO channel into equivalent parallel Gaussian channels for multi data stream transmission, as shown in figure 2.11. The application of the precoding matrix $\boldsymbol{V}$ at the evolved NodeB (eNodeB) before transmission and $\boldsymbol{U}^{*}$ at the UE during post-processing diagonalizes the channel, as shown in figure 2.11a. The data streams are represented by the non-zero singular values $\lambda_{1}, \lambda_{2} \ldots \lambda_{n_{\min }}$ along the diagonal of matrix $\boldsymbol{\Lambda}$.

The data streams or eigenchannels can be used to transmit independent information in each data stream if the channel conditions are supportive. A method used to allocate the signal power to the eigenchannels is known as the water filling method. The water filing 


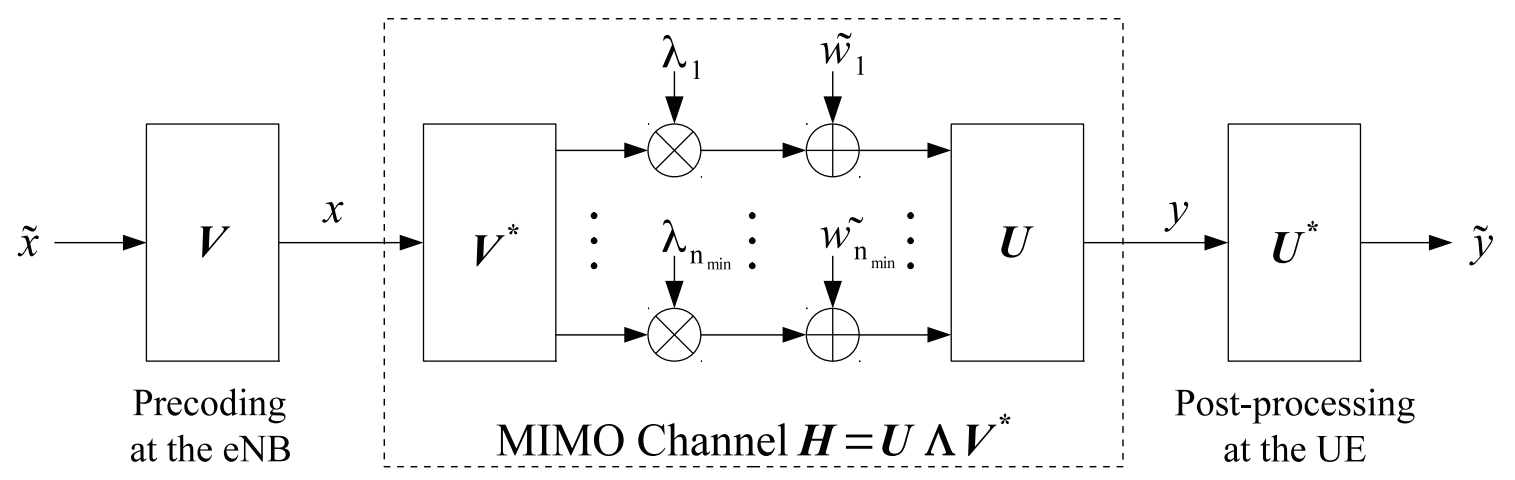

(a) Pre and Post-Processing Matrices Applied to MIMO Channel

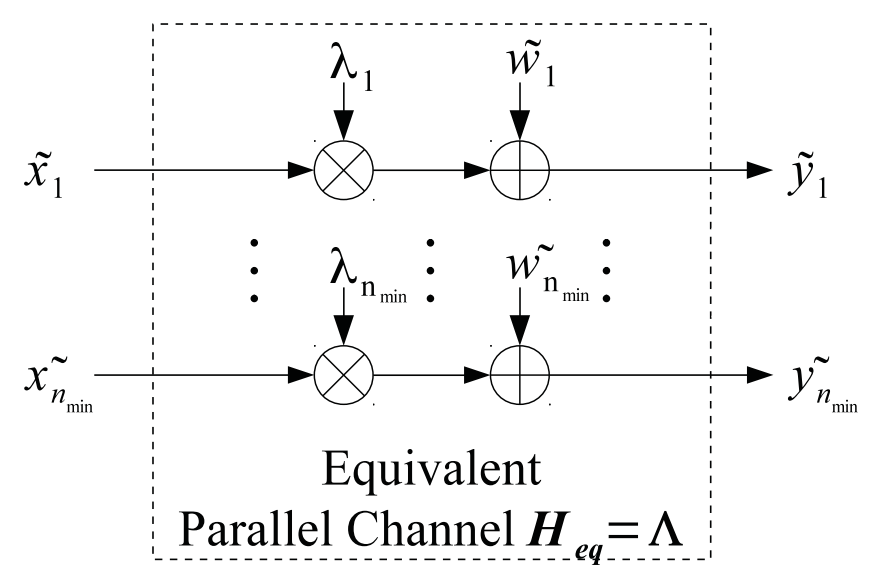

$$
\begin{aligned}
& \text { where } \Lambda=\left[\begin{array}{ccc}
\lambda_{1} & 0 & 0 \\
0 & \ddots & 0 \\
0 & 0 & \lambda_{n_{\min }}
\end{array}\right] \\
& \tilde{y}_{i}=\lambda_{i} \tilde{x}_{i}+\tilde{w}_{i}
\end{aligned}
$$

(b) Resulting Parallel Gaussian Channels After Application of SVD Pre and Post-Processing Matrices

Figure 2.11: Conversion of the MIMO Channel to Parallel Gaussian Channels using Singular Value Decomposition (SVD) 
allocates more power to the eigenchannels with stronger eigenmodes or greater singular values, which is equivalent to assigning more power to channels with the highest quality. The MIMO channel with the highest capacity is a channel where all the singular values are equal and the matrix spreads the transmit energy equally across all the base station antennas. Since the number of possible eigenchannels is limited to the dimensions of the MIMO channel matrix, the rank is limited by the number of transmit and receive antennas as shown in 2.17 .

$$
n_{\text {min }} \leq \operatorname{rank}\left\{H_{M I M O}\right\} \leq \min (N, M)
$$

The SVD and rank are exploited in LTE to drastically increase the wireless system's capacity. LTE transmission modes like spatial multiplexing are capable of transmitting multiple codewords over the channel when the SVD and rank reveal that multiple independent data streams are available in the channel. Single-user and multi-user spatial multiplexing is helping to expand the capacity of advanced wireless radio access networks but the capacity is heavily dependent on correlation between antennas and spatial characteristics of the channel that may limit or be overly optimistic about the presence of multiple data streams in the channel. High correlation between antennas results in a reduced rank channel matrix and reduced system capacity and vice-versa, low correlation between antennas and multi-path improves the chances to achieve high bit rates. Therefore, to develop advanced wireless systems, proper modeling of the antenna geometry, field pattern, correlation, polarization and the channel spatial characteristics is needed, otherwise, poor or exaggerated simulation results are possible.

The importance of the proper modeling of the MIMO channel model and the polarization and correlation between the physical antennas can be seen in equation 2.18 from [8], the capacity of a MIMO channel. In 2.18, if the signal to noise ratio (SNR) $\gamma$ and the number of transmit antennas $N$ are held constant, the result of the determinant increases as the rank of the channel matrix increases, corresponding to an increase in channel capacity and system throughput.

$$
C=\log _{2}\left[\operatorname{det}\left(\boldsymbol{I}_{M}+\frac{\gamma}{N} \boldsymbol{H} \boldsymbol{H}^{H}\right)\right]
$$

The increase in capacity has been shown to be related to the eigenvalues of $\boldsymbol{H} \boldsymbol{H}^{H}$ using singular value decomposition as shown in section 15.2-2 of [8]. The resulting capacity 
equation after SVD $\left(\boldsymbol{H} \boldsymbol{H}^{H}=\boldsymbol{Q} \Delta \boldsymbol{Q}^{H}\right)$ leads to the capacity for an equivalent channel composed of $r$ parallel Gaussian channels. The capacity of the equivalent $r$ parallel channels is shown in equation 2.19, where $r$ is the rank of the channel matrix $\boldsymbol{H}$.

$$
C=\sum_{i}^{r} \log _{2}\left(1+\frac{\gamma}{N} \lambda_{i}\right)
$$

Assuming independence between the antennas, the rank $r$ of the channel matrix is $n_{\min }$ from equation 2.17 and the MIMO channel capacity can be viewed as the sum of $r$ SISO channels which depend on the quality of each of the $i$ links, given by the eigenvalues $\lambda_{i}$. The rank and eigenchannel quality $\lambda_{i}$ are greatly dependent on the antenna correlation, channel model and require advanced spatial models that account for spatial characteristics and antenna geometry and correlation.

Similarly, the capacity of spatial multiplexing transmission modes used in LTE are highly dependent on the multiple data streams present in the channel as seen by the capacity equation in 2.19. Therefore, proper modeling of the antenna geometry, correlation and channel spatial characteristics is required to achieve simulation results that are representative of the results that would be achieved in a real wireless network.

To simulate a MIMO channel, the individual links between the transmitter and receiver can be modeled using multiple SISO channels. These methods are popular due to their simplicity and simulation speed but they lack the accurate polarization and correlation properties and spatial channel characteristics that are required to simulate multiple antenna systems like LTE. For this reason, the extended spatial channel model (SCME), a SoS based model, is used in this thesis to obtain the results presented in chapter 4. The SCME is covered in section 2.3.7 after introducing the physics used to model the received electric and the SoS modeling method.

\subsubsection{The Received Electric Field}

In its simplest form a single wireless communication link can be represented by a channel impulse response composed of a number of delayed taps which represent the multi-path in the environment. The small-scale fading caused by the channel impulse response is combined with shadowing and path loss to re-create realistic channel conditions using a simulator. This section introduces the physical model behind the small-scale fading multipath since it is the component of the channel model that leads to a better understanding of the spatial characteristics of the channel. 
Wireless propagating signals are varying electric fields which are emit from the transmitter's antennas and propagate in the wireless channel until they are absorbed or received at the receiver. The electric fields can be represented by rays that propagate in the environment from the transmitter to the receiver as shown in figure 2.12.

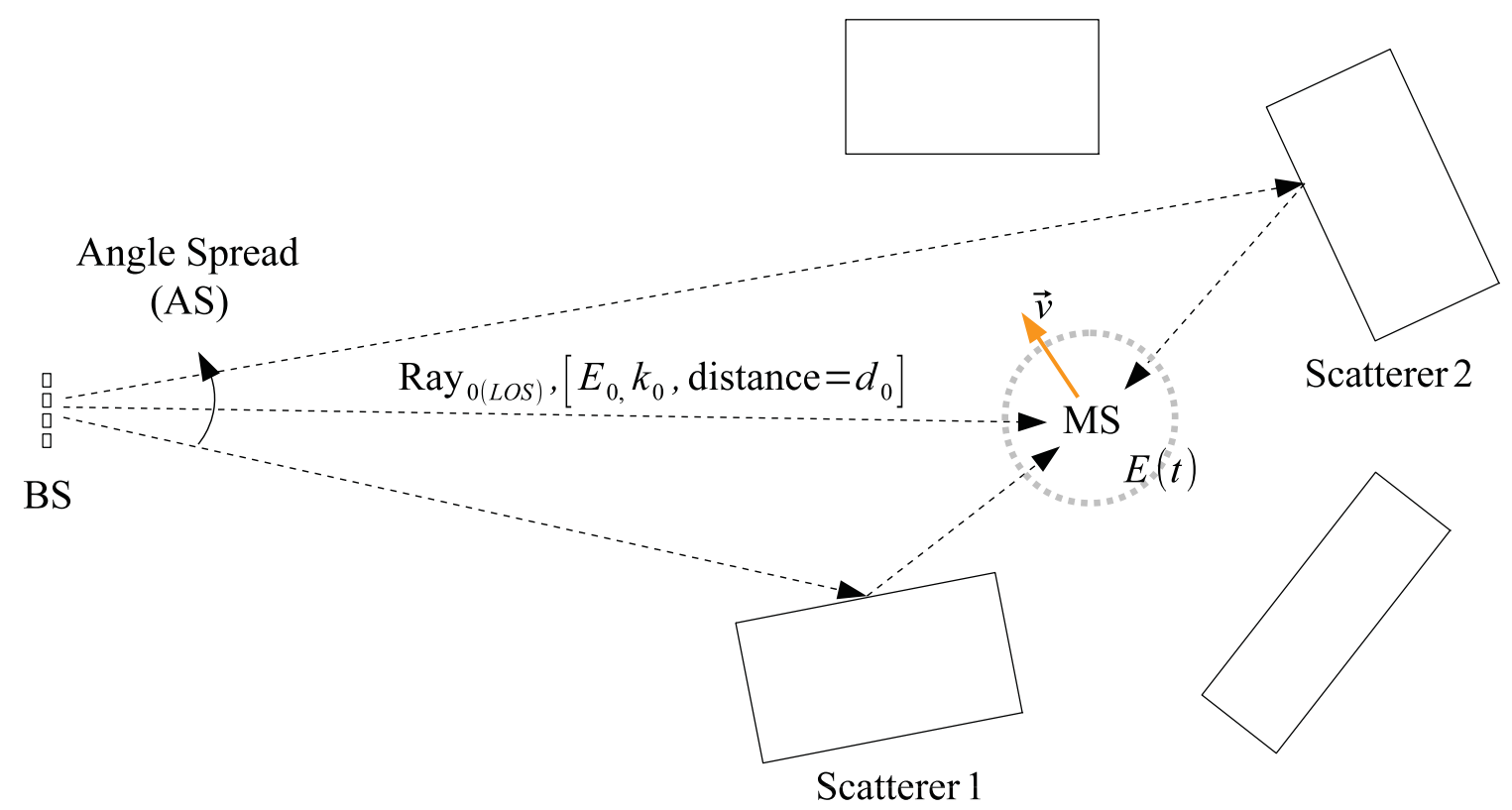

Figure 2.12: The Received Electric Field using the Ray Model

The propagating rays are affected by squattering objects in the environment and take different paths to reach the receiver. The paths cause the electric fields to arrive at the receiver with varying phases due to the different path length each signal follows. The small-scale fading effects are caused by the superposition of multiple complex electric fields $E(t)$ at the receiver. The electric field generated at the receiver by a single ray is shown in equation 2.20. The received field $E(t)$ is caused by the field $E_{0}$ generated at the transmitter. The propagating field also arrives at the receiver with a phase related to the distance $d$ traveled by the ray and the wave number $k_{0}$.

$$
E=E_{0} \cdot e^{-j k_{0} d}
$$

When the received signal is the combination of two paths, the delays are $\tau_{1}=d_{1} / c_{0}$ and $\tau_{2}=d_{2} / c_{0}$ and the total received electric field $E(\vec{r})$ due to the combined fields with amplitudes $E_{1}$ and $E_{2}$ is shown in equation 2.21. 


$$
E(\vec{r})=E_{1} \cdot e^{-j k_{1} \vec{r}}+E_{2} \cdot e^{-j k_{2} \vec{r}}
$$

Generalizing the total electric field $E(\vec{r})$ for multiple paths results in equation 2.22.

$$
E(\vec{r})=\sum_{i=1}^{N} E_{i} \cdot e^{-j k_{i} \vec{r}}
$$

Note that a single path in figure 2.12 can be represented by 2.23 using Euler's formula. Here $f_{c}$ is the carrier frequency of the propagating wave, in Hertz $(\mathrm{Hz})$, and $k_{0}$ and $d$ are the wave number and the propagation distance of an arbitrary path from equation 2.21.

$$
E(t)=E_{0} \cdot \cos \left(2 \pi f_{c} t-k_{0} d\right)
$$

By replacing $d$ with the reference distance $d_{0}$ plus the additional distance of a longer path, such that $d=d_{0}+v t$, equation 2.24 is obtained.

$$
E(t)=E_{0} \cdot \cos \left[2 \pi t f_{c}-k_{0}\left(d_{0}+v t\right)\right]
$$

In addition, $k=2 \pi / \lambda$ can be substituted for $k_{0}$, where $\lambda$ is the wavelength of the propagating signal. Performing simple manipulations results in equation 2.25. In equation 2.25 we can observe the effect of the Doppler shift on the propagating signal by the receiver velocity component in the excerpt $\left(f_{c}-v / \lambda\right)$.

$$
E(t)=E_{0} \cdot \cos \left[2 \pi t\left(f_{c}-\frac{v}{\lambda}\right)-\frac{2 \pi d_{0}}{\lambda}\right]
$$

Finally, the substitutions to simplify equation 2.25 substitutions for the phase $(\phi=$ $\left.-2 \pi d_{0} / \lambda\right)$ and Doppler frequency $\left(f_{d}=v / \lambda\right)$ lead to equation 2.26.

$$
E(t)=E_{0} \cdot \cos \left(2 \pi f_{c} t-2 \pi f_{m} \cos (\gamma) t+\varphi\right)
$$

In order to introduce spatial effects caused by the angle of arrival (AoA) of the received signals, the Doppler effect $f_{d}$ for the $i^{\text {in }}$ received field is replaced with $f_{d}=f_{m} \cos \left(\gamma_{i}\right)$, where the maximum Doppler frequency $f_{m}$ is multiplied by the cosine of the angle of arrival $\gamma_{i}$ of the $i^{\text {ih }}$ received wave as shown in equation 2.27.

The small-scale fading physical model is completed by summing the electric fields of multiple waves at the receiver as shown in equation 2.27. The received electric field is the sum of the electric field amplitudes $\left|a_{i}\right|$ for each incident wave, with varying phases. 


$$
E(t)=\sum_{i=1}^{N}\left|a_{i}\right| \cos \left(2 \pi f_{c} t-2 \pi f_{m} \cos \left(\gamma_{i}\right) t+\varphi_{i}\right)
$$

Factoring out the in-phase $I(t)$ and quadrature $Q(t)$ components leads to the basis for Clarke's SoS model [5] and a number of more recently proposed SoS models which will be briefly discussed.

$$
\begin{gathered}
E_{B P}(t)=I(t) \cos \left(2 \pi f_{c} t\right)-Q(t) \sin \left(2 \pi f_{c} t\right) \\
I(t)=\sum_{i=1}^{N}\left|a_{i}\right| \cos \left(-2 \pi f_{m} \cos \left(\gamma_{i}\right) t+\varphi_{i}\right) \\
Q(t)=\sum_{i=1}^{N}\left|a_{i}\right| \sin \left(-2 \pi f_{m} \cos \left(\gamma_{i}\right) t+\varphi_{i}\right)
\end{gathered}
$$

The physical equations presented in 2.28, 2.29 and 2.30 are discussed in further detail in the following sections where the physical representation is adapted into wireless channel models proposed by Clarke [5], Jakes [9], Pop and Beaulieu [10], Xiao, Zheng and Beaulieu [2] and more recently the 3GPP and the Wireless World Initiative New Radio (WINNER) organizations.

The early models are designed to be as efficient as possible to reduce simulation time based on the available processing power at the time they were presented. As computing power and the complexity of wireless systems increase, the models need to evolve to represent the channel's spatial characteristics to better represent complex MIMO systems with higher fidelity. A brief discussion of some of the changes proposed by experts will show how the evolution has lead to the state of the art spatial channel models developed by the 3GPP and the WINNER organizations for the evaluation of 4G systems.

\subsubsection{Clarke's Reference Model}

In Clarke's classical paper [5], R. H. Clarke proposed his mobile radio signal model based on the physical equations he presented, developed in section 2.3.4 and summarized in equations 2.28, 2.29 and 2.30. A number of assumptions were made by Clarke limiting the usable scenarios of his model to narrow band, frequency flat and non-line-of-sight (NLOS) propagation scenarios but the model remains a reference for improved models even today. 
The model proposed by Clarke [5] is briefly discussed here.

Clarke's linear time variant (LTV) channel model, $h(t, \tau)$, is based on multi-path propagation arriving at the receiver. The $L$ multi-path components forming his channel model are characterized by a delays $\tau_{l}$ and complex amplitudes $g_{l}$ as shown in equation 2.31.

$$
h(t, \tau)=\sum_{l=0}^{L-1} g_{l}(t) e^{-j 2 \pi f \tau_{l}}
$$

The $L$ channel complex amplitudes in the CIR are generated using Clarke's scattering model which sums $N$ incident waves arriving at the receiver, known as sub-paths. Each complex valued path $g(t)$ is the normalized sum of equal amplitude sub-paths as shown equation 2.32, where the in-phase and quadrature components are given in equations 2.33 and 2.34 .

$$
\begin{gathered}
g(t)=g_{c}(t)+j g_{s}(t) \\
g_{c}(t)=\frac{1}{\sqrt{N}} \sum_{n=1}^{N} \cos \left(2 \pi f_{m} \cos \alpha_{n}+\phi_{n}\right) \\
g_{s}(t)=\frac{1}{\sqrt{N}} \sum_{n=1}^{N} \sin \left(2 \pi f_{m} \cos \alpha_{n}+\phi_{n}\right)
\end{gathered}
$$

Clarke's assumptions for the model given by the set of equations in 2.33-2.34 are that the amplitudes are constant and the angles of arrival $\alpha_{n}$ and the phases $\phi_{n}$ are independent and uniformly distributed over the angles 0 to $2 \pi$ as illustrated in figure 2.13 .

The random variables were carefully selected based on work previously done by Gilbert [11], Young [12] and Jakes and Reudink [13]. Clarke noted that equation 2.33 and 2.34 were Gaussian at sufficiently large $N$ based on the central limit theorem, resulting in a Rayleigh distributed channel envelope. When a strong LOS component existed, the distribution became Ricean. At sufficiently large values of $N$ the statistical model presented by Clarke, given the assumptions, appeared to be a good predictor of the wireless radio channel. The statistical properties of the model, described by $2.35-2.38$ from [14], demonstrate important key characteristics of the model. 


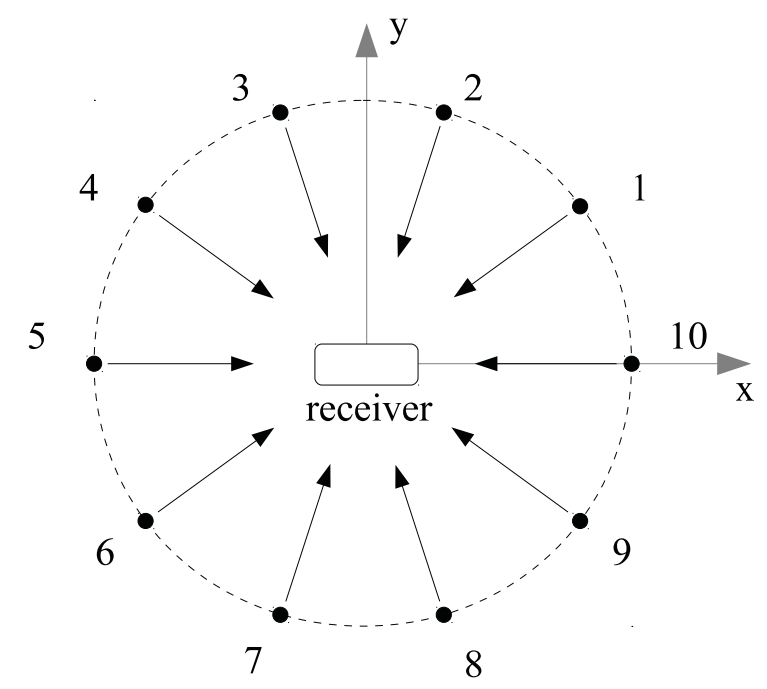

Figure 2.13: A uniformly distributed set of incident wave fronts, with angle of arrivals $\alpha_{n}$, arriving at the UE

$$
\begin{gathered}
R_{g_{c}} R_{g_{c}}(\tau)=R_{g_{s}} R_{g_{s}}(\tau)=\frac{1}{2} J_{0}\left(2 \pi f_{m} \tau\right) \\
R_{g_{c}} R_{g_{s}}(\tau)=R_{g_{s}} R_{g_{c}}(\tau)=0 \\
R_{g g}(\tau)=J_{0}\left(2 \pi f_{m} \tau\right) \\
R_{|g|} R_{|g|^{2}}=1+J_{0}^{2}\left(2 \pi f_{d} \tau\right)-\frac{J_{0}^{2}\left(2 \pi f_{d} \tau\right)}{N}
\end{gathered}
$$

For example, the cross-correlation of the in-phase and quadrature components shown in equation 2.36 demonstrates that the real $g_{c}$ and imaginary $g_{s}$ components of the paths are independent.

The auto-correlation of the complex channel realization shown in equation 2.37 is used to find the classical Doppler spectrum derived by Jakes' in [9].

The Doppler spectra is the probability density function, or distribution, of Doppler shifts also known as the Doppler spread. While there are various definitions for the Doppler spectrum, the Jakes U-shaped Doppler spectrum in equation 2.39 from [9] is a particularly well-known version. In equation 2.39, $J_{0}$ is the zero ${ }^{\text {th }}$-order Bessel function of the first kind and $f_{m}$ the maximum Doppler frequency. 


$$
S_{f}= \begin{cases}\frac{1}{\pi f_{m}} \cdot \frac{1}{\sqrt{1-\left(f / f_{m}\right)^{2}}} & |f| \leq f_{m} \\ 0 & |f|>f_{m}\end{cases}
$$

Jake's U-shaped Doppler spectrum from equation 2.39 is shown in figure 2.14.

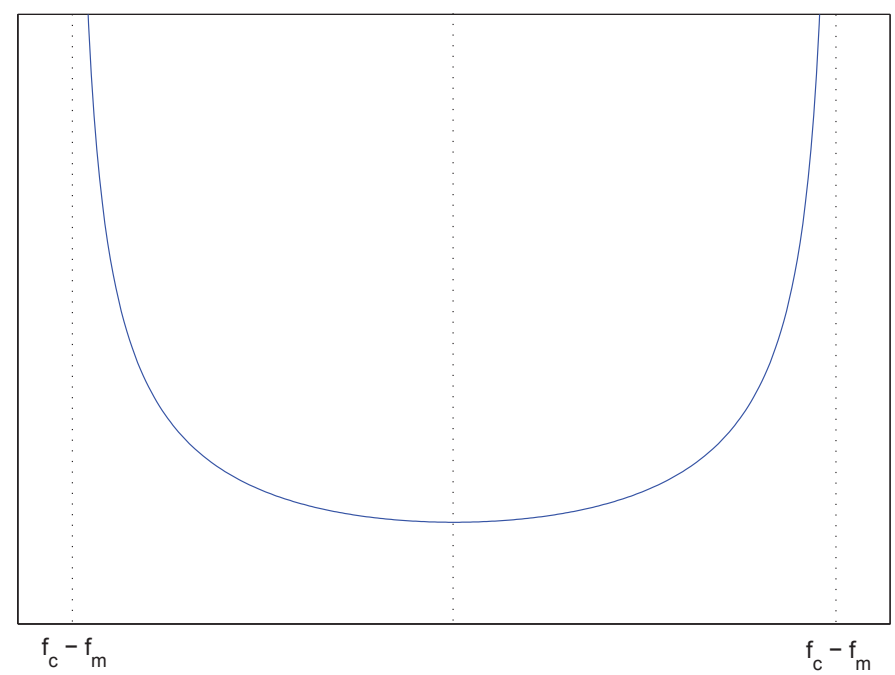

Figure 2.14: Illustration of the Jakes U-Shaped Doppler Spectrum

Doppler and the variations of the channel have been shown to be linked to motion at the MS. The channel variations in the time domain can be related to the receiver movement with respect to the transmitter via the Doppler shift experienced at the receiver. The relationship between the receiver velocity $v$, the direction of travel with respect to the base station $\theta_{i}$ and the rate of change of the channel $f_{i}$ for the $i^{\text {th }}$ path is given in equation 2.40 from [15].

$$
f_{i}=f_{m} \cos \left(\theta_{i}\right)=v \cdot \cos \left(\theta_{i}\right) \lambda
$$

The physical model presented in this section and Clarke's mobile channel fading process remains a reference for improved models. However, due to the relatively high values of $N$ required by Clarke's model, the model is used only as a benchmark for lower complexity models, such as Jakes' model and improved versions of Jake's model. 


\subsubsection{Jakes' Model}

Jakes' model is a lower complexity model similar to Clarke's SoS method. Jakes model attempts to exploit symmetry to lower the complexity of the model and maintain similar statistics to Clarke's reference model while reducing the number of Doppler frequencies.

The complete definition of Jakes' model is given by the complex amplitude $g(t)$ listed in appendix B.1. Jakes reduced the number of sinusoid oscillators by fixing the attenuation of the $n^{\text {th }}$ path to $1 / \sqrt{N}$ and defining the number of oscillators $N$ as $N=4 M+2$ reducing the number of distinct oscillators to $M+1$ [10], where $M \geq 8$. The in-phase and quadrature components for Jakes' model, shown in equation 2.42 and 2.43 are considered a reduced realization of Clarke's model given in section 2.3.5 since the model converges with as little as $M=8$ oscillators used during simulation.

In Jakes' model, $\omega_{m}$ is the maximum Doppler frequency in radians per second. The maximum Doppler frequency is given by $\omega_{m}=2 \pi v / \lambda$, where $v$ is the velocity of the receiver, $\lambda$ is the wavelength at the carrier frequency and both $\beta_{n}$ and $\beta_{M+1}$ are angles of arrival (AoA).

$$
\begin{gathered}
\tilde{g}(t)=\tilde{g}_{c}(t)+j \tilde{g}_{s}(t) \\
\tilde{g}_{c}(t)=\sqrt{2} \cos \beta_{M+1} \cos \omega_{m} t+2 \sum_{n=1}^{M} \cos \beta_{n} \cos \omega_{n} t \\
\tilde{g}_{s}(t)=\sqrt{2} \sin \beta_{M+1} \cos \omega_{m} t+2 \sum_{n=1}^{M} \cos \beta_{n} \sin \omega_{n} t
\end{gathered}
$$

The diagram ${ }^{1}$ for Jakes' model shows the realization of the channel's fading process $\tilde{g}(t)$ and the shared $M+1$ low frequency oscillators that generate the in-phase $\tilde{g}_{c}(t)$ and quadrature components $\tilde{g}_{s}(t)$ of the process.

\footnotetext{
${ }^{1}$ Note that normalization constants are not shown in the diagram in figure 2.15 to avoid cluttering the diagram.
} 


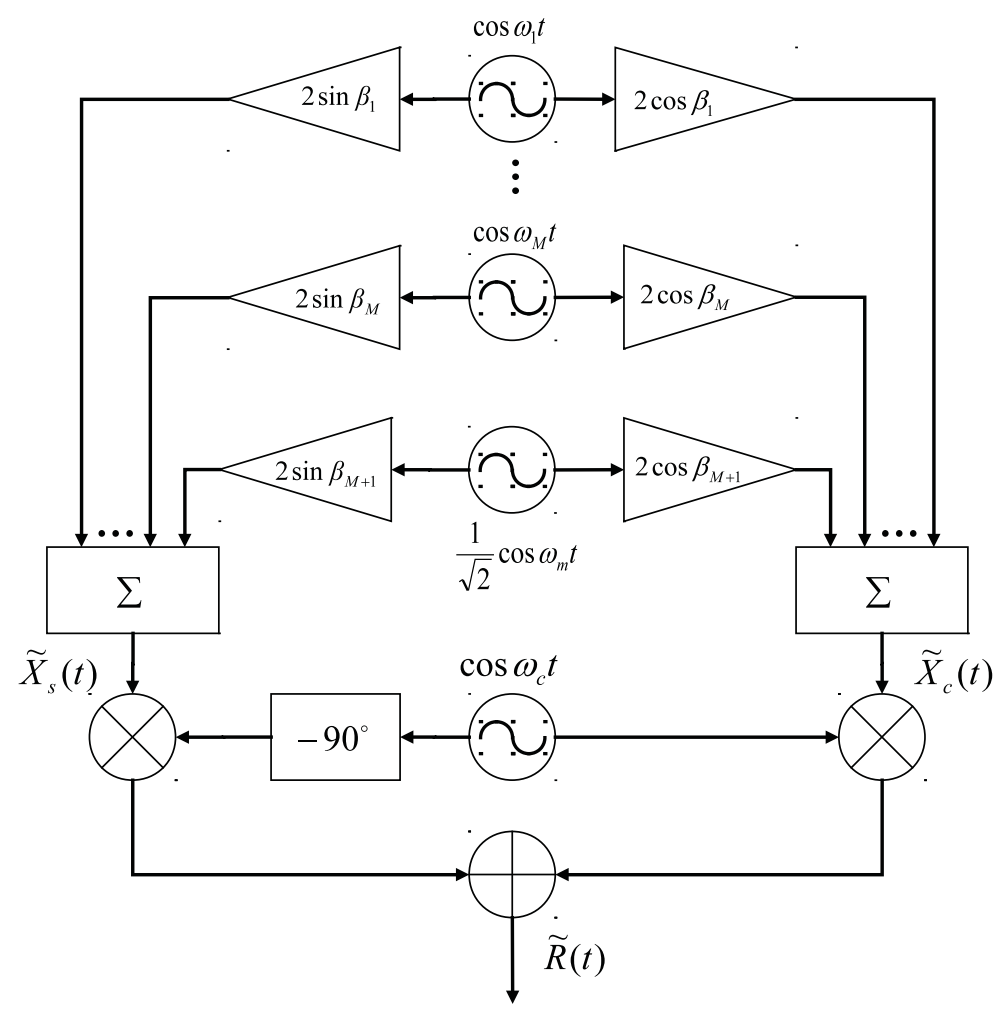

Figure 2.15: Diagram of Jakes' model 
The key differences between Jakes' model and Clarke's reference model are:

- Angle of arrival " $a_{n}$ " and " $b_{n}$ " distribution:

- Clarke's model: $[-\pi, \pi)$.

- Jakes' model uses positive Doppler frequencies only to reduce the number of sinusoids to $M$.

- Jakes' model is deterministic, which means when all parameters (angle of arrival, path attenuation and phases) are fixed each simulation run is identical and the parameters remain constant during the whole simulation run.

- Amplitudes of the sinusoids in Jakes' model were selected to produce zero crosscorrelation between in-phase and quadrature components to remain similar to Clarkes model.

- Jakes' model is more efficient for simulation due to fewer sinusoids $\left(M_{\text {Jakes }}<\right.$ $N_{\text {Clarke }}$ ).

Despite being a widely used model for simulation, key statistics in Jakes' model do not match the reference model when the number of oscillators $M$ is small. In [10] it was also revealed that the process $g(t)$ is non-stationary, not wide sense stationary (WSS) and the variance of the fading process is time varying. While Jakes model may be efficient to implement in hardware or software, the use of common low frequency Doppler oscillators made by Jakes is not valid and noted in [10]. In addition, Pop and Beaulieu [10] also note that fixing the path attenuation, angles of arrival and phases for the duration of the simulation does not model real world results, however, they admit that fixing the path attenuation and angles of arrival is reasonable.

\section{Improved Jakes’ Model}

Improvements to Jakes' model have been proposed by a number of experts. The goal of the proposed improvements remains to generate channel coefficients for simulation which exhibit statistics that are equivalent to those of Clarke's model while maintaining low complexity for reasonable simulation times.

In [10], Pop and Beaulieu proposed an improved model that solves the WSS problem by drawing the oscillator phases from a uniform independent and identically distributed (i.i.d.) distribution over the interval $[0,2 \pi)$. Using random variables for the oscillator phases eliminates the correlation of the oscillators and the authors show that the probability 
density function (PDF) is no longer time dependent. The improved WSS channel process converges toward the desired Rayleigh flat fading signal with uniform phase over $[0,2 \pi)$.

In addition, a recent publication [2] by Xiao, Zheng and Beaulieu proposes an improved SoS model, shown in equations 2.44-2.46, that rapidly converges to the reference model faster than the reference model itself for $N=8$. The angles of arrival $\alpha_{n}$ of the new fading process $Y(t)$ are redefined as $\alpha_{n}=2 \pi n+\theta_{n} / N$ for $n=1,2, \ldots, N$.

$$
\begin{gathered}
Y(t)=Y_{c}(t)+j Y_{s}(t) \\
Y_{c}(t)=\frac{1}{\sqrt{N}} \sum_{n=1}^{N} \cos \left(\omega_{d} t \cos \alpha_{n}+\phi_{n}\right) \\
Y_{s}(t)=\frac{1}{\sqrt{N}} \sum_{n=1}^{N} \sin \left(\omega_{d} t \cos \alpha_{n}+\phi_{n}\right)
\end{gathered}
$$

In the in-phase and quadrature equations, 2.45 and 2.46, the phases $\phi_{n}$ of the received waves are uniformly distributed random variables similar to the previously proposed improvement to Jakes's model. However, in [2] the phases $\phi_{n}$ are distributed over $[-\pi, \pi)$ and a new random variable, $\theta_{n}$, is introduced in order to randomize the angle of arrival $\alpha_{n}$. The small increase in simulator complexity is a small price to pay for the significant improvement in the simulator quality which can be seeing in the convergence of the autocorrelation in figure 2.16.

A short summary of the strengths of the improved model by Xiao et al. [2] are listed here:

- The introduction of $\theta_{n}$, an independent and uniformly distributed random variable over $[-\pi, \pi)$ used to randomize the angles of arrival, ensures better statistical quality.

- The angles of arrival are no longer fixed $\alpha_{n}$ (such as in Jakes' model).

- The same number of angle of arrival values $\alpha_{n}$ are generated as Clarke's model, however, for small $N$, the proposed model ensures a more uniform distribution.

- The first order statistics, $R_{Y_{c} Y_{c}}(\tau)$ and $R_{Y_{s} Y_{s}}(\tau), R_{Y_{c} Y_{s}}(\tau)$ and $R_{Y_{s} Y_{c}}(\tau)$, and $R_{Y Y}(\tau)$, of the proposed model are the same as the reference model.

The goal of each newly proposed model is to reproduce realistic wireless channel effects as closely as possible while keeping the simulation complexity low. With the tremendous 


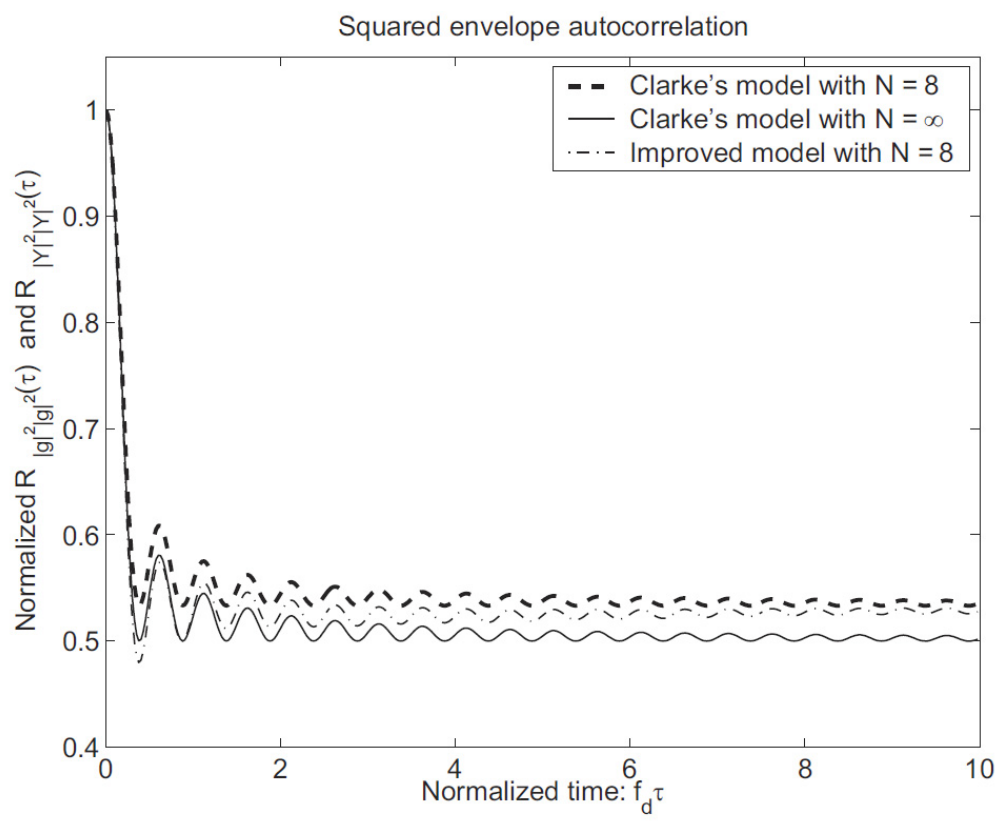

Figure 2.16: Comparison from figure 1 of [2] of the Autocorrelation of the Improved SoS Simulator Proposed in [2] vs the Reference Model

growth in computing power and the optimization of wireless channel models two things are possible. The first is better modeling of more channel characteristics such as azimuth angle of departure (AoD), and angle of arrival (AoA), antenna field patterns and polarization. The second is the improved simulation time of complex modern simulators which gives researchers excellent insight into the performance of complex multi-layered single-user MIMO (SU-MIMO) and multi-user MIMO (MU-MIMO) systems within a very reasonable amount of time.

In this section, the theory of wave propagation and the models presented by Clarke, Jakes and Pop, Beaulieu et al have been discussed since they are the basis for more advanced geometry based spatial channel models. Section 2.3.7 introduces the 3GPP extended spatial channel model, referred to as SCME for short. The 3GPP SCME model is the basis for the link level simulations presented in chapter 4 of this thesis.

\subsubsection{Spatial Channel Model}

The need for spatial channel models became apparent when MIMO technology began to gain traction in $\mathrm{c}$ in the wireless industry. The advances in MIMO applied to commercial 
wireless networks promised higher spectrum efficiency which could be used to increase throughput and quality of service demanded by wireless consumers. In order to enable the design of these new wireless access technologies, new channel models with more spatial characteristics were required to evaluate MIMO related features.

The design goals for the spatial channel models were to:

- Enable researchers to improve the spectral efficiency of wireless networks by designing systems that make use of the actual channel conditions.

- Properly model multiple antenna MIMO technology, antenna field patterns, correlation and polarization.

- Offer a larger selection of wireless environments for simulation.

- Separate the antennas from the channel model to allow for any antenna setup to be used.

In 2003, the first release of the 3GPP standard TR 25.996 R6.1.0 [16] included the 3GPP $\backslash 3^{\text {rd }}$ Generation Partnership Project 2 (3GPP2) spatial channel model. The first model was geared toward a single narrow frequency band in the $2 \mathrm{GHz}$ range and wireless outdoorto-outdoor propagation scenarios. Improved models were later developed in 2005 by Baum, Hansen, Del Galdo, Milojevic et al. [17] to extend the SCM based on work accomplished by WINNER while aiming to keep the SCME backward compatible with the 3GPP SCM. The work started based on the immediate need for models to evaluate beyond $3 \mathrm{G}$ systems (B3G) systems.

The goals for the SCME were:

- Increase the bandwidth to $100 \mathrm{MHz}$ while keeping the SCME backward compatible with the SCM using intra-cluster delay spread zero-delay-spread-cluster or zerodelay sub-cluster (ZDSC).

- Add path loss model support for the $5 \mathrm{GHz}$ frequency band.

- Time varying large-scale parameters (LSPs) and LOS and k-factor models.

The SCME was later modified for 3GPP LTE simulation purposes. WINNER continued its work in 2005 to develop the WINNER phase I model, named WINNER channel model - phase 1 (WIM1) which resulted in a larger number of scenarios. WINNER later extended the WINNER models further and released the model under the name WINNER channel model - phase 2 (WIM2). The WINNER models included measurement 
based extensions to support $100 \mathrm{MHz}$ of usable bandwidth which is a requirement for LTE-Advanced wideband systems. In addition, WINNER added outdoor BS to indoor MS scenarios, cross-correlation between the LSPs and time-evolution improvements over the $\mathrm{SCM}^{2}$ and $\mathrm{SCME}^{3}$.

All of the models discussed (the SCM, SCME, WIM1 and WIM2) are considered stochastically controlled geometric models based on the SoS modeling method. The models are stochastically controlled because several random variables are drawn and then used to sum sinusoids to form single MPCs to describe the channel impulse response. The principles behind the SCM, the SCME and the WINNER models are the same but do offer varying feature sets as shown in table 2.1.

Table 2.1: Feature Comparison for SCM, SCME, WIM1, WIM2 Spatial Channel Models

\begin{tabular}{lcccc}
\hline \multicolumn{1}{c}{ Feature } & SCM & SCME & WIM I & WIM II \\
\hline \hline Bandwidth & $5 \mathrm{MHz}$ & $100 \mathrm{MHz}^{*}$ & $100 \mathrm{MHz}$ & $100 \mathrm{MHz}$ \\
Indoor scenarios & & $\checkmark$ & $\checkmark$ \\
$\begin{array}{l}\text { Outdoor-to-indoor } \\
\text { and indoor-to-outdoor }\end{array}$ & & $\checkmark$ & $\checkmark$ \\
AoA $\backslash$ AoD elevation & & $\checkmark$ & $\checkmark$ \\
$\begin{array}{l}\text { Intra-cluster delay spread (DS) } \\
\text { Tapped-delay-line (TDL) model }\end{array}$ & & $\checkmark$ & $\checkmark$ & $\checkmark$ \\
LSP cross-correlation & & $\checkmark$ & $\checkmark$ \\
Parameter time-evolution & & & $\checkmark$ \\
\hline
\end{tabular}

In chapter 4 of this thesis the SCME is used as the primary tool for the evaluation of the LTE downlink performance, therefore, the features and inner workings of the SCME are presented in this section. For a detailed description of all the features of the SCME the reader can consult the 3GPP technical report TR 25.996 [3] and [17] which discusses the modifications made to the SCM to arrive at the SCME.

\footnotetext{
${ }^{2}$ The SCM did not include time evolution of the LSPs.

${ }^{3}$ The SCME included continuous evolution for the first time but its implementation drastically increased simulation time.
} 


\section{Bulk Parameters and the Concept of the Drop}

All of the variations of the spatial channel models described in the introduction of section 2.3.7 use the concept of a drop. A drop is a term used to describe a simulation run where the channel's fast fading process is allowed to evolve with time but the large-scale parameters (LSPs) of the channel are held constant. This is typically referred to as a drop because the high level large-scale parameters, also known as "bulk parameters", can be fixed for the purposes of evaluating the performance achieved by the MS at a given time and point in space $(x, y, z)$ as shown in figure 2.17. The high level parameters are considered significantly different from one drop to another, therefore, the large-scale parameters can be considered constant and independent during a short period of time.

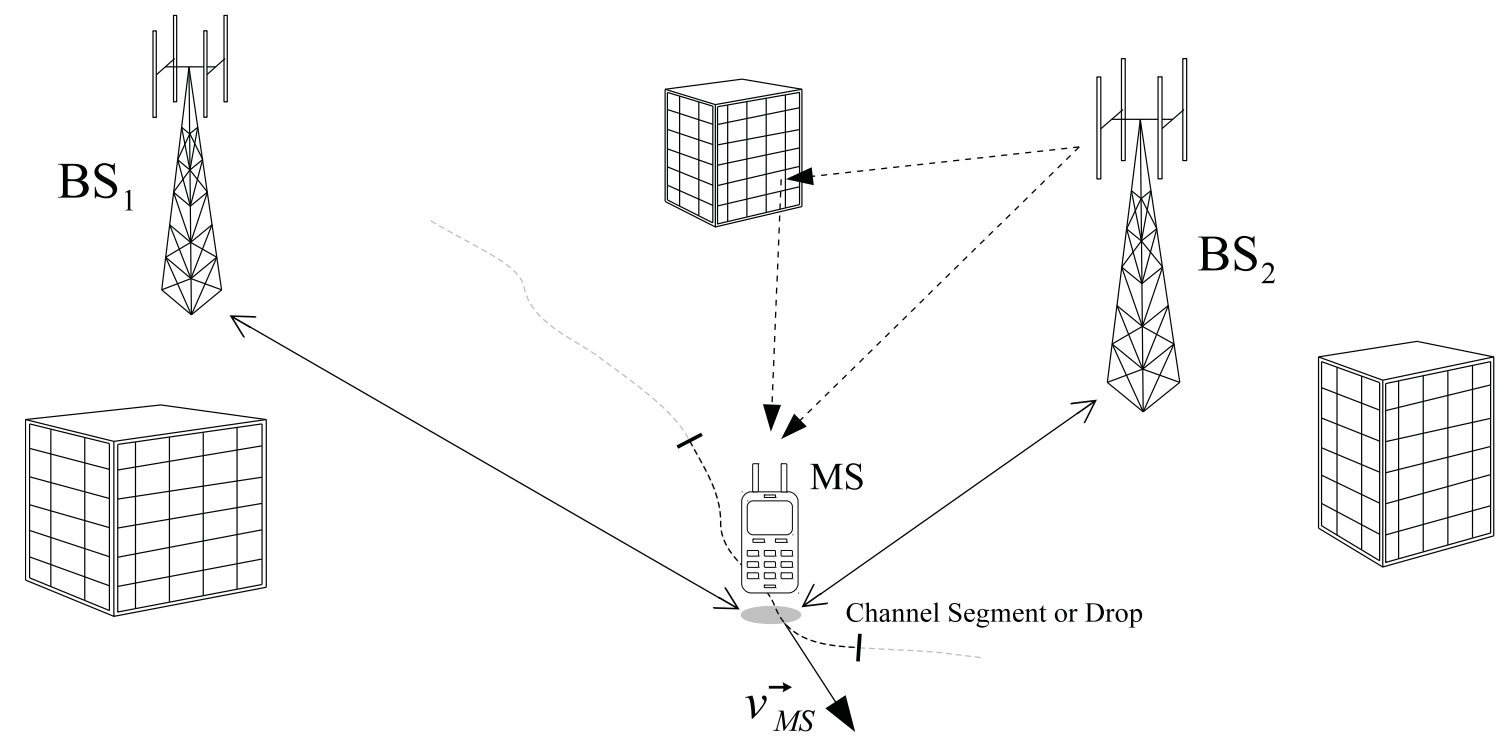

Figure 2.17: The Drop (or Channel Segment)

The location of the base station and the UE in a drop can be random or fixed by the user. In a typical simulation the BS locations are specified by the user and the UE location is randomly generated by the simulation software. This type of simulation allows the user to evaluate the UE performance across the serving cell and measure average performance or plot particular distributions such as the distribution of SNR or UE throughput. Randomizing the UE location and channel parameters is typically done in system level simulations and provides a holistic view of the performance under many conditions.

In this thesis, the UE location was fixed in the simulations in order to compare the 
performance of different antennas, transmission modes and impairments. The drop (ie. large-scale parameters) is held constant for proper comparisons by fixing the simulator's random number generator seeds. This ensures the drop and generated channel are the same for each simulation and each curve. The controlled simulation scenario permits the exact comparison of 2 or more link level algorithms, modes or impairments because only the desired input parameters are changed.

\section{Time-Evolution of Large-Scale Parameter and the Channel Segment}

In recently developed models, such as the SCME and WIM2, the simulator does allow for the large-scale parameters to evolve over time, however, a simulation run is still considered a drop since the large-scale parameters drift from their original values and the BS and UE locations do not change. The drifting is used to represent UE movement within the environment. As the channel coefficients are generated the drifting large-scale parameters control the probability distributions of the low-level parameters in an attempt to model the non-stationary properties of a wireless channel.

The large-scale parameters that are allowed to drift are:

- Log-normal shadowing (shadow fading (SF)): $\sigma_{S F}$

- Delay spread (DS): $\sigma_{D S}$

- Angle spread (angle spread (AS)): $\sigma_{A S}$

Of the three evolving large-scale parameters, log-normal shadowing and delay spread have already been introduced in section 2.1.1 and 2.1.2. In the case of shadow fading, a log-normal random variable is used to model the large variations in the average received signal power caused by the obstruction of the LOS signal component. The delay spread is a measure of the time dispersion of the channel which results in fading in the frequency domain and is a design consideration when selecting the $\mathrm{CP}$ length and pilot spacing in the frequency domain.

The mean angle spread $E\left(\sigma_{A S}\right)$ of the multi-path departing the base station antennas affects the angles of departure of the paths. The angular parameters are shown in the spatial channel's ray model shown in figure 2.18. One sub-path is shown relative to the cluster of paths. In the ray model in figure 2.18, the angle of departure is labeled $\delta_{n, A o D}$ for the path shown in the. The mean angle spread $E\left(\sigma_{A S}\right)$ is the expected angular deviation of the $N$ angles of departure $\delta_{n, A o D}$ generated by the channel model for each of the $N$ paths. The 
choice of the mean angle spread $E\left(\sigma_{A S}\right)$ sets the distribution parameters $\mu_{A S}$ and $\varepsilon_{A S}$ for the angle spread $\sigma_{A S}$ log-normal random variable in order to achieve an expected angle spread $E\left(\sigma_{A S}\right)$ of either $8^{\circ}$ or $15^{\circ}$ for the $N$ paths at the base station. The mean angle spread $E\left(\sigma_{A S}\right)$ determines the correlation between the $N$ paths and multi-path richness of the channel. All three LSPs (shadow fading, delay spread and angle spread) are evolved over time in the SCME to model the non-stationary nature of the drop.

The short term UE motion causing the large-scale parameters to drift is encapsulated in the concept of the drop but it is sometimes more appropriately named a channel segment due to the motion and changing channel that it represents. The channel segment is represented by a small section of the total UE trajectory, shown in figure 2.17.

The SCME also includes time evolution of some of the low-level parameters during a simulation and is therefore referred to as a continuous time-evolution channel model because the parameters evolve at each simulation step. The SCME allows the angles of arrival $\delta_{n, A o D}$ of the multi-path components and the sub-path delays to drift but holds the angles of departure $\delta_{n, A o D}$ at the BS constant. The reason for holding the angles of departure $\delta_{n, A o D}$ constant is that the geometry, consisting of the base station and the UE, is considered fixed during a drop and the angles of departure $\delta_{n, A o D}$ of the paths departing the BS do not change. On the other hand, the time-evolution and UE motion causes changes in the angle of arrivals $\delta_{n, A o A}$ and tap delays $\tau_{n}$ of the multi-path components over a short period of time. For the interested reader, the update equations for the angles of arrival and tap delays of the multi-path components can be found in section 2a of [17].

\section{Scenario and Environment Parameters}

The large-scale parameters, also known as bulk parameters, are determined by the particular scenario selected by the user. The SCME has three possible choices for the scenario which determine the large-scale and low-level parameters.

The three possible scenarios and their corresponding inter-site distance (ISD) and BS antenna heights are:

- Suburban macro (ISD of $3 \mathrm{~km}, \mathrm{BS}$ antennas above roof-top height)

- Urban macro (ISD of $3 \mathrm{~km}, \mathrm{BS}$ antennas above roof-top height)

- Urban micro (ISD less than $1 \mathrm{~km}, \mathrm{BS}$ antennas at roof-top height)

The three scenarios determine the parameters used to control the spatial channel model. 
The parameters for each of the three possible scenarios are listed in table 5.1 of [3]. The scenario selected for the simulations in this thesis is the urban macro scenario due to its support for up to $100 \mathrm{MHz}$ of bandwidth ${ }^{4}$ in the $2-6 \mathrm{GHz}$ frequency range. The environment parameters for the urban macro scenario are summarized in table 2.2.

Table 2.2: Environment Parameters for the Urban Macro Scenario

\begin{tabular}{|c|c|c|}
\hline Channel Parameter & Symbol & Value \\
\hline $\begin{array}{l}\text { Mean angle spread (AS) } \\
\text { at the BS }\end{array}$ & $E\left(\sigma_{A S}\right)$ & $\begin{array}{l}\text { Low angular spreading: } E\left(\sigma_{A S}\right)=8^{\circ} \\
\text { High angular spreading: } E\left(\sigma_{A S}\right)=15^{\circ}\end{array}$ \\
\hline $\begin{array}{l}\text { Angle spread (AS) at BS as } \\
\text { log-normal random variable }\end{array}$ & & $\begin{array}{l}\sigma_{A S}=10^{\varepsilon_{A S} x+\mu_{A S}}, x \sim \eta(0,1) \\
\text { For } E\left(\sigma_{A S}\right)=8^{\circ}, \text { let } \mu_{A S}=0.81, \varepsilon_{A S}=0.34 \\
\text { For } E\left(\sigma_{A S}\right)=15^{\circ}, \text { let } \mu_{A S}=1.18, \varepsilon_{A S}=0.21\end{array}$ \\
\hline Ratio of $\sigma_{A o D}$ to $\sigma_{A S}$ & $r_{A S}$ & $r_{A S}=\sigma_{A o D} / \sigma_{A S}=1.3$ \\
\hline Per-path AS at the BS & & $2 \operatorname{deg}$ (fixed) \\
\hline BS per-path AoD distribution & & $\eta \sim\left(0, \sigma_{A o D}^{2}\right)$, where $\sigma_{A o D}=r_{A S} \sigma_{A S}$ \\
\hline Mean AS at the MS & $E\left(\sigma_{A S}, M S\right)$ & $68^{\circ}$ \\
\hline Per-path AS at the MS & & $35^{\circ}$ (fixed) \\
\hline MS per-path AoA distribution & & $\eta \sim\left(0, \sigma_{A o A}^{2}(P r)\right)$ \\
\hline Mean RMS delay spread & $E\left(\sigma_{D S}\right)$ & $0.65 \mu s$ \\
\hline $\begin{array}{l}\text { Delay spread (DS) as } \\
\text { log-normal random variable }\end{array}$ & $\sigma_{D S}$ & $\begin{array}{l}\sigma_{D S}=10^{\varepsilon_{D S} x+\mu_{D S}}, x \sim \eta(0,1) \\
\text { where } \mu_{D S}=-6.18 \text { and } \varepsilon_{D S}=0.18\end{array}$ \\
\hline Ratio of $\sigma_{\text {delays }}$ to $\sigma_{D S}$ & $r_{D S}$ & $r_{D S}=\sigma_{\text {delays }} / \sigma_{D S}=1.7$ \\
\hline $\begin{array}{l}\text { Log-normal shadowing } \\
\text { standard deviation }\end{array}$ & $\sigma_{S F}$ & $8 \mathrm{~dB}$ \\
\hline Path-loss model (dB) & $P L d B(d)$ & See equation 2.2 \\
\hline
\end{tabular}

In addition to the choice of the scenario, the mean angle spread $E\left(\sigma_{A S}\right)$ of the BS multipath components can be set to low or high when the urban macro scenario is selected. The

\footnotetext{
${ }^{4} \mathrm{SCME}$ was artificially extended to $100 \mathrm{MHz}$ from $5 \mathrm{MHz}$ by using 3-4 mid-paths per cluster ZDSC, also keeping the SCME backward compatible with the SCM.
} 
mean angle spread $E\left(\sigma_{A S}\right)$ influences the deviation of the angles of departure of the multipath components leaving the base station. A mean angle spread $E\left(\sigma_{A S}\right)$ value of $8^{\circ}$ (low angular spreading) was used in the simulations in this thesis.

\section{BS and MS Angular Parameters used in the SCME}

The SCME is based on the ray and sum-of-sinusoids model described in section 2.3.4 and is known as a stochastically controlled geometric model since the model is based on the geometry of the cell layout and ray propagation representing the received electric field encapsulated in the SoS simulator. The model is stochastically controlled since the large-scale parameters and a number of low-level random variables are generated based on varying distributions which model the wireless channel.

The ray model used in the SCME is shown in figure 2.18. The ray diagram is used to more realistically model the physics behind the propagating wireless radio signals. In figure 2.18 one of the $M$ sub-paths within one of the $N$ paths is shown departing the base station, reflecting or scattering off an object and ending at the receiver. The angular parameters associated with the $m^{\text {th }}$ sub-path forming the $n^{\text {th }}$ path are relative to the BS and MS array broadside and the line-of-sight signal component. The angular parameters that define the ray model in figure 2.18 are summarized in table 2.3 .

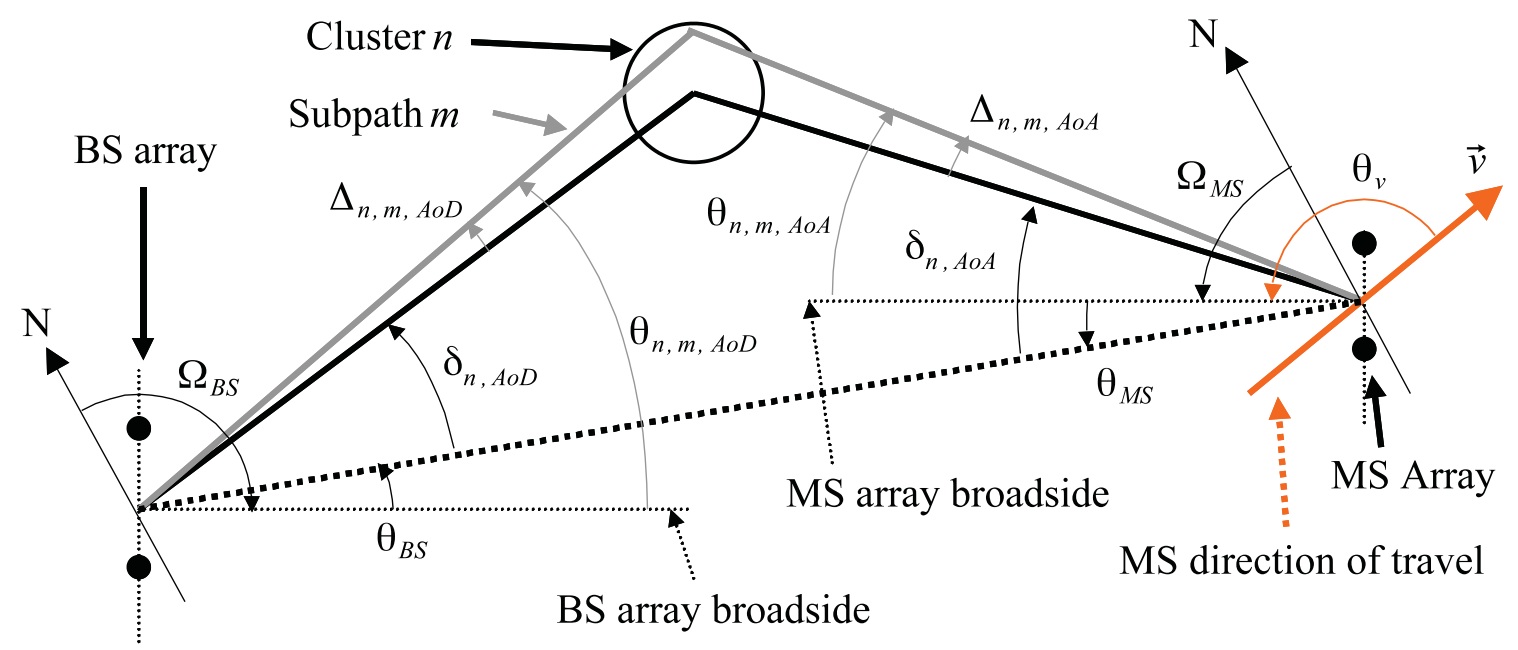

Figure 2.18: Diagram from [3] of the Sub-paths and Clusters used in the Spatial Channel Model 
Table 2.3: Description of the Spatial Channel Model Angular Parameters

\begin{tabular}{|c|c|}
\hline Symbol & Description \\
\hline$n$ & $\begin{array}{l}\text { The } n^{\text {th }} \text { path or cluster (equivalent to the number of taps in the CIR) } \\
\text { where } n=1 \ldots N \text { and } N=6\end{array}$ \\
\hline$m$ & The $m^{\text {th }}$ sub-path within the $n^{\text {th }}$ path (cluster), where $m=1 \ldots M$ and $M=20$ \\
\hline \multicolumn{2}{|c|}{ Base Station } \\
\hline$\Omega_{B S}$ & The BS array broadside orientation with respect to absolute North \\
\hline$\theta_{B S}$ & The angle between the BS-MS LOS and the BS array broadside \\
\hline$\delta_{n, A o D}$ & The angle of departure for the $n^{\text {th }}$ path with respect to the LOS component \\
\hline$\Delta_{n, m, A o D}$ & The offset for the $m^{\text {th }}$ sub-path of the $n^{\text {th }}$ path with respect to $\delta_{n, A o D}$ \\
\hline$\theta_{n, m, A o D}$ & $\begin{array}{l}\text { The absolute AoD for the } m^{\text {th }} \text { sub-path of the } n^{\text {th }} \text { path at the BS } \\
\text { with respect to the BS array broadside }\end{array}$ \\
\hline \multicolumn{2}{|c|}{ Mobile Station } \\
\hline$\Omega_{M S}$ & The MS array broadside orientation with respect to absolute North \\
\hline$\theta_{M S}$ & The angle between the BS-MS LOS and the MS array broadside \\
\hline$\delta_{n, A o A}$ & The angle of arrival for the $n^{\text {th }}$ path with respect to the LOS component \\
\hline$\Delta_{n, m, A o A}$ & The offset for the $m^{\text {th }}$ sub-path of the $n^{\text {th }}$ path with respect to $\delta_{n, A O A}$ \\
\hline$\theta_{n, m, A o A}$ & $\begin{array}{l}\text { The absolute AoA for the } m^{\text {th }} \text { sub-path of the } n^{\text {th }} \text { path at the MS } \\
\text { with respect to the MS array broadside }\end{array}$ \\
\hline$\vec{v}$ & The MS velocity vector \\
\hline$\theta_{v}$ & The angle of velocity vector $\vec{v}$ with respect to the MS array broadside \\
\hline
\end{tabular}


The angular parameters shown in table 2.3 are used to model the angles of arrival and departure of the sub-paths forming the multi-path components. The $M$ sub-paths per path represent the wave-fronts arriving at the receiver's antennas with varying phases, delays and angles of departure and arrival. The sub-path and path angular parameters are determined by the LSPs and distributions defined when the user selects the scenario. The $M=20$ subpaths are then summed together to form the $N=6$ paths, as in the sum-of-sinusoids models, to build the 6 tap channel impulse response used to model the multi-path that causes the small-scale fading present in the wireless channel. The $N$ paths, obtained by summing the sub-paths are also known as clusters in the spatial channel model and represent one channel impulse response between the $s^{\text {th }}$ base station antenna element and the $u^{\text {th }}$ MS antenna element.

The SCME keeps the spatial channel model's complexity low by drawing statistical values only where the extra accuracy justifies the added complexity. For example, the $M$ sub-paths constituting the paths are assigned fixed relative offset angles $\Delta_{n, m, A o D}$ and $\Delta_{n, m, A o A}$ about the angle of departure $\delta_{n, A o D}$ and the angle of arrival $\delta_{n, A o A}$ of the $n^{\text {th }}$ path. The predefined fixed sub-path offset angles $\Delta_{n, m, A o D}$ and $\Delta_{n, m, A o A}$, found in table 5.2 of [3], reduce the complexity and decrease simulation time while still maintaining statistical validity.

\section{Parameter Generation for the Urban Macro Scenario}

The generation of the channel impulse response $h_{u, s, n}(t)$ between a transmitter and a receiver at a particular point in time $t$ is a thirteen step process described in detail in section 5.3.1 of [3] for the system level simulator model. The user provides the simulator the cell layout, desired scenario, BS and MS antenna configuration and the UE velocity vector. The simulation software prepares the distributions for the random variables representing the delays $\tau_{n}$, path powers $P_{n}$, phases $\Phi_{n, m}^{(x, y)}$ and angles of departure $\delta_{n, A o D}$ and arrival $\delta_{n, A o A}$. The $\mathrm{BS}$ and MS angles and delays are ordered and paired based on increasing values and the antenna gains for each of the $M=20$ sub-paths of the $N=6$ paths can then be determined. The parameters generated by the simulator are then provided to the channel model equation in figure 2.19 which generates the complex fading taps in the channel impulse response for each transmitter-receiver link $(s, u)$ by summing the sub-path sinusoids for each path. The parameters used in the equation in figure 2.19 to generate the CIR are summarized in table 2.4 .

The parameters for the antennas in the channel coefficient generating equation are the 


$$
h_{u, s, n}(t)=\sqrt{\frac{P_{n} \sigma_{S F}}{M}} \sum_{m=1}^{M}\left(\begin{array}{l}
{\left[\begin{array}{l}
\chi_{B S}^{(v)}\left(\theta_{n, m, A o D}\right) \\
\chi_{B S}^{(h)}\left(\theta_{n, m, A o D}\right)
\end{array}\right]^{T}\left[\begin{array}{cc}
\exp \left(j \Phi_{n, m}^{(v, v)}\right) & \sqrt{r_{n 1}} \exp \left(j \Phi_{n, m}^{(v, h)}\right) \\
\sqrt{r_{n 2}} \exp \left(j \Phi_{n, m}^{(h, v)}\right) & \exp \left(j \Phi_{n, m}^{(h, h)}\right)
\end{array}\right]\left[\begin{array}{l}
\chi_{M S}^{(v)}\left(\theta_{n, m, A o A}\right) \\
\chi_{M S}^{(h)}\left(\theta_{n, m, A o A}\right)
\end{array}\right] \times} \\
\exp \left(j k d_{s} \sin \left(\theta_{n, m, A o D}\right)\right) \times \exp \left(j k d_{u} \sin \left(\theta_{n, m, A o A}\right)\right) \times \exp \left(j k\|\mathbf{v}\| \cos \left(\theta_{n, m, A o A}-\theta_{v}\right) t\right)
\end{array}\right)
$$

Figure 2.19: Spatial Channel Model

Table 2.4: Description of the Parameters used in the SCME's Channel TDL Generating Equation

\begin{tabular}{ll}
\hline \multicolumn{1}{c}{ Symbol } & Number of sub-paths \\
\hline \hline$M$ & Log-normal shadow fading \\
$\sigma_{S F}$ & Power of the $n^{\text {th }}$ path \\
$P_{n}$ & AoD and AoA for the $m^{\text {th }}$ sub-path \\
$\theta_{n, m, A o D}, \theta_{n, m, A o A}$ & The wave number \\
$k$ & Distance between the antenna elements and \\
$d_{s}, d_{u}$ & the reference BS and MS antenna element \\
& Phase of the $m^{\text {th }}$ sub-path of the $n^{\text {th }}$ path for the $x$ \\
$\Phi_{n, m}^{(x, y)}$ & polarization at the BS and $y$ polarization at the MS \\
& Magnitude and angle of the MS velocity vector \\
$\|v\|, \theta_{v}$ & BS antenna complex response for the $v$ and $h$ polarizations \\
$\chi_{B S}^{(v)}\left(\theta_{n, m, A o D}\right), \chi_{B S}^{(h)}\left(\theta_{n, m, A o D}\right)$ & MS antenna complex response for the $v$ and $h$ polarizations \\
$\chi_{M S}^{(v)}\left(\theta_{n, m, A o A}\right), \chi_{M S}^{(h)}\left(\theta_{n, m, A o A}\right)$ & $\begin{array}{l}\text { Random variables representing the ratio of the power } \\
r_{n 1}, r_{n 2}\end{array}$ \\
& of the $n^{\text {th }}$ wave leaving the BS in one polarization \\
and arriving at the MS in the other polarization
\end{tabular}


antenna field patterns $\chi_{B S}^{(h)}\left(\theta_{n, m, A o D}\right), \chi_{B S}^{(v)}\left(\theta_{n, m, A o D}\right), \chi_{M S}^{(h)}\left(\theta_{n, m, A o A}\right)$ and $\chi_{M S}^{(v)}\left(\theta_{n, m, A o A}\right)$ based on the sub-path angles of departure $\theta_{n, m, A o D}$ and angles of arrival $\theta_{n, m, A o D}$, the antenna element distance $d_{s}$ and $d_{u}$ and the cross-polarization discrimination ratio modeled using the crosspolarization signal power ratios $r_{n 1}$ and $r_{n 2}$. Polarization and antenna field pattern modeling are described in detail in section 5.5.1 and 4.5 of [3], respectively.

Doppler is taken into account using the magnitude $\|\vec{v}\|$ and angle $\theta_{v}$ of the velocity vector $\vec{v}$ and the wave number $k$ (which contains the wavelength and carrier frequency).

The spatial channel model is characterized by a high number of statistically drawn random variables used to represent the propagating electric fields in the wireless environment. In step 1-3 in section 5.3.1 of [3] the powers, delays, phases, AoDs and AoAs are randomly selected. The mean angle spread $E\left(\sigma_{A S}\right)$ selected by the user is used to achieve a desired angle spread $\sigma_{A S}$ for the $N$ paths giving the user the ability to simulate different scenarios such as 3 -sector vs 6 -sector cell where the angle spread is expected to be wider in the 3-sector cell. However, for simplicity, the sub-path angle spread at the BS within each path is fixed. The quality of the model and the model's ability to accurately handle spatial correlation and polarization based on the input by the user cell layout and physical antenna description ensures the MIMO channel produced is representative of the actual BS and MS antennas and cell layout being modeled by the user.

The SCME's physical accuracy, the improved bandwidth over the first SCM, time evolution introducing drifting and other features makes the SCME model suitable for evaluating LTE's spatial multiplexing schemes using a variety of antenna spacings and polarization configurations.

\subsubsection{Simulation Model}

For each simulation run, the cell layout, the UE location and the UE velocity are provided by the user. The generation of the channel coefficients is accomplished in three general steps. The first step is the selection of the propagation scenario and the angular spreading which will be used to determine the environment parameters discussed in section 2.3.7. The second step is performed by the simulator and includes drawing on various distributions to determine the large-scale paramaters (eg. shadow fading, delay spread) and then following the thirteen steps outlined in section 5 of [3] to determine the path powers, path delays and generate the angles of arrival and departure of the $M$ sub-paths within each path. The $n^{\text {th }}$ complex channel tap for transmitter $s$ and receiver $u$ are then generated using the equation in figure 2.19 and applying the per-path power $P_{n}$ and shadow fading $\sigma_{S F}$. 
The spatial channel model produces a $U$ by $S$ by $N$ matrix of complex coefficients, where $U$ is the number of receivers, $S$ is the number of transmitters and $N$ is the number of taps, known as the MIMO channel matrix. The MIMO channel matrix is used with additive white Gaussian noise to simulate the links in the wireless channel. The MIMO channel matrix $\mathbf{H}_{\text {MIMo }}(\mathbf{t})$ at the instant in time $t$ can also be viewed as a $U$ by $S$ matrix of channel impulse responses modeling the channels between the base station transmitting antennas and the MS receiving antennas.

The simulation model for a single transmitter-receiver link $(s, u)$ is shown in figure 2.20. In general, the simulations in this thesis are used to achieve throughput versus SNR performance curves and require the simulator to normalize the transmit power and control the amount of additive white Gaussian noise in order to achieve a given SNR for each point plotted within the desired SNR range. The model shown in figure 2.20 is for a single transmitter-receiver link $(s, u)$ but it is extended to MIMO channels by generating channel impulse responses for all of the transmitter-receiver links that exist between the base station antennas and the MS antenna array.

\subsection{Long Term Evolution}

Long Term Evolution (LTE) is the name given by 3GPP to the set of standards for the widely adopted next generation wireless standards based on OFDM technology. OFDM systems are designed to balance the need for a robust wireless system with the demands of high bit rate applications. LTE is no different then any other system and in the process of standardization, the LTE parameters were chosen to overcome typical channel effects, such as dispersion, inter-carrier interference (ICI) and ISI discussed in section 2.1 and 2.2.

The design rules followed in the LTE standardization process are:

- To avoid ISI, choose a cyclic prefix length $T_{c p} \geq$ delay spread $T_{d}$ of the channel.

- To keep ICI due to Doppler shift low, select a cyclic prefix length $T_{c p}>$ the delay spread $T_{d}$.

- Select the sub-carrier frequency spacing $\Delta f>>$ the maximum Doppler shift $f_{m}$.

- Choose $T_{s} \gg T_{c p}$ for spectral efficiency.

LTE systems are based on the OFDMA scheme which is very well suited for the high bit rate systems being standardized in technologies such as LTE and its advanced version 


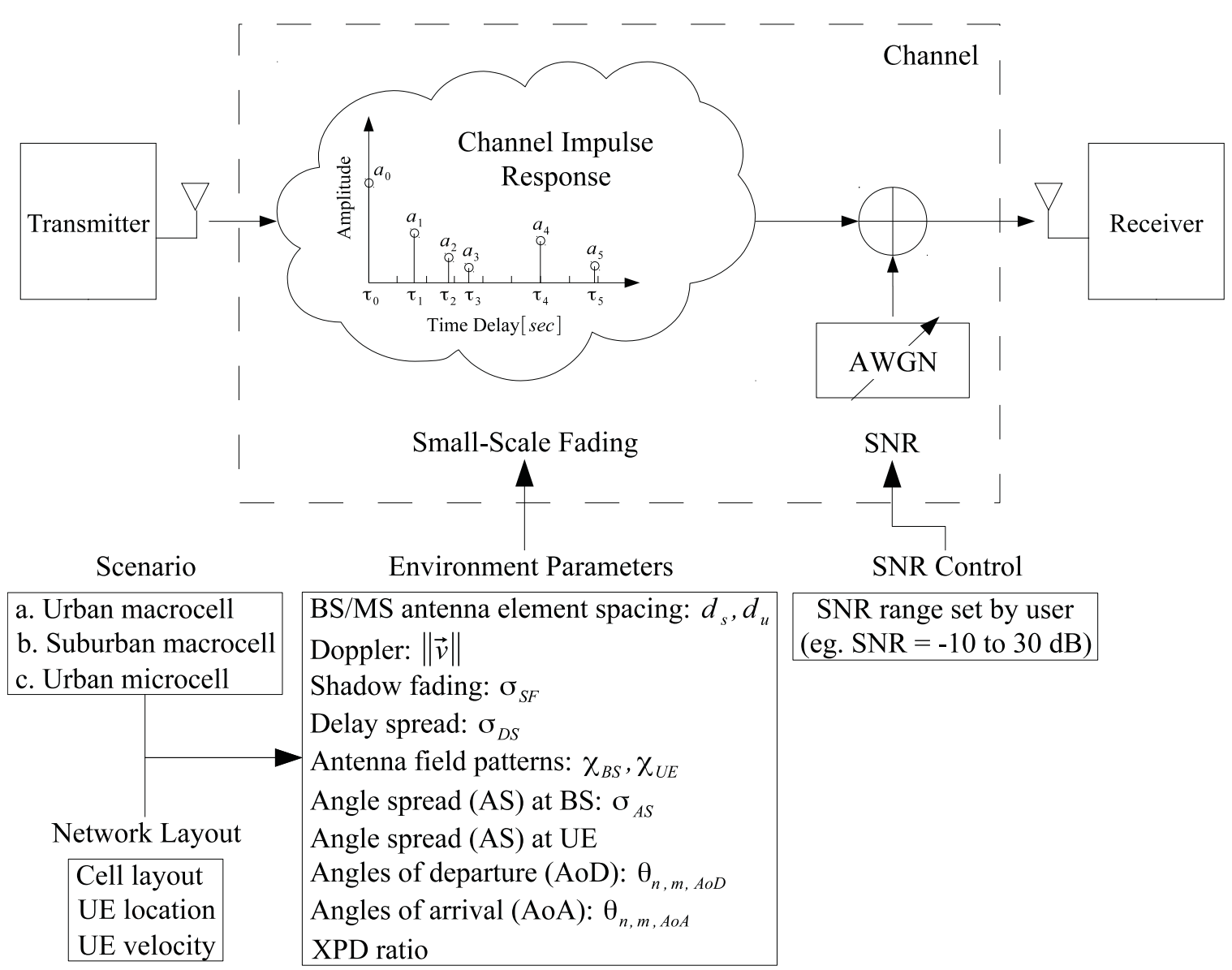

Figure 2.20: Simulation Model: Channel Generation and Spatial Channel Parameters Used to Model a SISO Link 
called LTE-Advanced. The simulations in this thesis are focused on the performance of the LTE physical downlink shared channel (PDSCH) which is the channel used to transfer datacentric information to the $\mathrm{UE}^{5}$ from the serving base station. The OFDM system design parameters for the LTE physical downlink shared channel are highlighted in table 2.5.

Table 2.5: Typical LTE System Parameters

\begin{tabular}{lcl}
\hline \multicolumn{1}{c}{ System Parameter } & Cyclic Prefix & \multicolumn{1}{c}{ Value } \\
\hline \hline \multirow{2}{*}{ Sub-carrier Spacing } & Normal & $15 \mathrm{kHz}$ \\
& Extended & $7.5 \mathrm{kHz}$ \\
\hline \multirow{2}{*}{ Cyclic Prefix } & Normal & $5.2 \mu \mathrm{sec}$ (first symbol), $4.7 \mu \mathrm{sec}$ \\
& Extended & $16.7 \mu \mathrm{sec}$ (scenarios with long delay spreads) \\
\hline Sampling Frequency & & Factor of the base frequency $3.84 \mathrm{MHz}$ \\
\hline System Bandwidth & & $1.4,3,5,10,15,20 \mathrm{MHz}$ \\
\hline
\end{tabular}

In the wireless network, the base station has the authority over the LTE air interface and assigns time and frequency domain resources to the UE due to its position in the network. The time-frequency resources are connections that are created between the base station and one or more UEs when data is scheduled to be transferred. The PDSCH resources are allocated after all other higher priority resources are reserved (eg. control channels, reference signals). The PDSCH resources can be addressed to a single UE (unicast) or a group of UEs (multicast) using a cell radio network temporary identifier (C-RNTI). The UEs are informed of the allocated PDSCH time-frequency resources by reading specific bitmaps sent by the BS on the physical downlink control channel (physical downlink control channel (PDCCH)). The bitmaps used to inform the UE of the PDSCH allocation are known as downlink control information (DCI) messages and are a key component of LTE's frequency selective scheduling feature used to overcome poor channel conditions as discussed in section 2.4.6.

In section 2.4.1, the transmission chain used to transmit the OFDM symbols carrying the PDSCH data will be presented prior to discussing the LTE transmission modes used for in the simulations.

\footnotetext{
${ }^{5}$ The term user equipment (UE) is used in the context of LTE wireless radio access systems.
} 


\subsubsection{OFDM Transmitter Chain}

Figure 2.21 is a block diagram of the LTE OFDM signal chain used by the LTE base stations for OFDM symbol transmission. The transmission chain is composed of several blocks that take a set of bits produced by higher layers and converts the bits to OFDM symbols for transmission over the wireless channel.

The first step in the process of OFDM symbol generation is code block segmentation. The bit stream is separated into blocks of bits and cyclic redundancy check (CRC) parity bits are attached to the blocks for error detection. Forward error correction is made possible by the coding and rate matching blocks using the LTE turbo encoder which helps overcome the errors introduced by the channel using interleaving and convolutional encoding. At the receiver the redundant information is used by the iterative turbo decoder to recover the original codewords and messages transmit by the base station. After channel coding, the bits are scrambled with identification numbers and then modulated onto sub-carriers and mapped across the frequency domain of the channel. The inverse fast Fourier transform (IFFT) is used to obtain the OFDM symbol in the time domain. The symbol is pre-pended with the cyclic prefix, just before transmission, to permit the receiver to remove the portion of the ISI that falls within the CP.

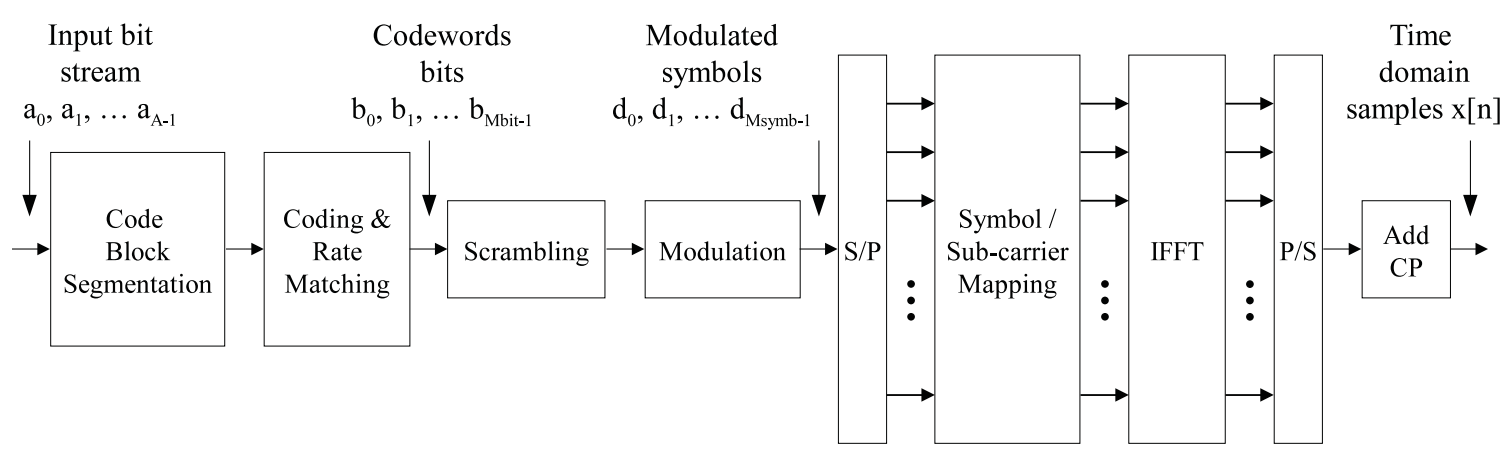

Figure 2.21: LTE OFDM Transmitter Chain with Modulation and Coding, IFFT and CP Addition for Single Layer Transmissions

The transmission chain seen in figure 2.21 takes one set of message bits and produces a single codeword. Since the layer mapping block is not present, a single layer is implied in figure 2.21. Layers are used to map one or more codewords to layers across the available antenna ports, if the channel and transmission mode supports multiple layers. Figure 
2.21 can be extended to include multiple layers and precoding by using multiple transmission chains in parallel and linking them with the layer mapping block as shown in figure 2.22. The layer mapping and precoding steps enable the advanced multi-antenna spatial multiplexing features, such as multiple codewords and beamforming, that are of interest in chapter 4 of this thesis and are discussed in section 2.5.3 and 2.5.4.

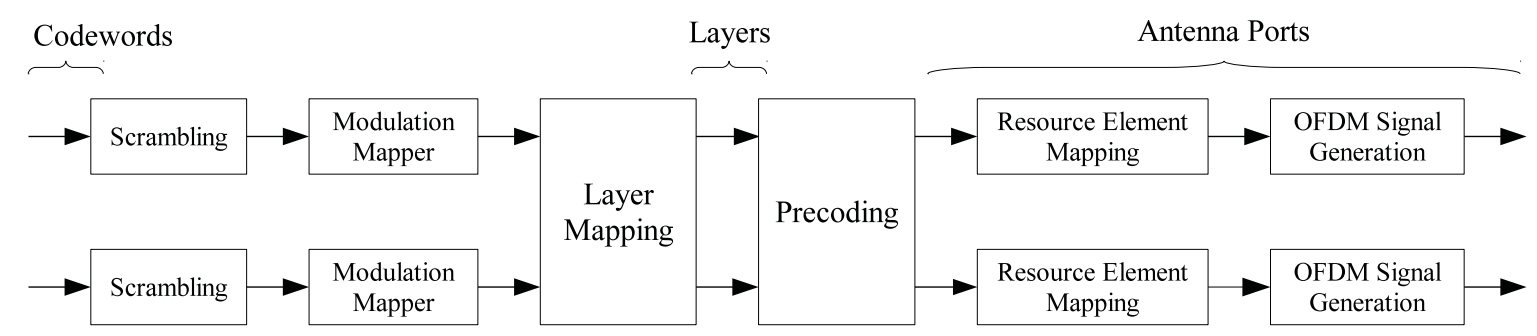

Figure 2.22: LTE OFDM Transmitter Chain with Layer Mapping, Precoding and Port Mapping

\section{Code Block Segmentation and CRC Attachment}

The process by which the bit stream is converted to an RF signal, at the physical layer, begins with the raw bit stream $a_{0}, a_{1}, \ldots, a_{A-1}$ provided as an output by the MAC layer. At the Code Block Segmentation and Code Block CRC Attachment stage, a 24-bit cyclic generator polynomial $g_{C R C 24 A}(D)$ is used to calculate and append a set of $L=24$ CRC parity bits to the input bit stream $a_{0}, a_{1}, \ldots, a_{A-1}$. The result after CRC attachment is a CRC protected bit sequence of length $B=A+24$. If the length of the CRC protected bit sequence does not exceed the maximum code block length $Z$, defined as $Z=6144$ in [18], then no code block segmentation is required and the protected bit stream is passed to the Coding and Rate Matching block. On the other hand, if the protected bit sequence length $B$ is longer then the maximum code block length $Z$, then code block segmentation and additional CRC parity bits are appended on an individual code block basis. The long bit stream is segmented into $C=\lceil B /(Z-L)\rceil$ code blocks and then individually protected with additional CRC parity bits calculated with the cyclic generator polynomial $g_{C R C 24 B}(D)$. The code block are then passed to the next block where coding and rate matching take place. 


\section{Coding and Rate Matching (RM)}

The Coding and Rate Matching block performs the turbo encoding, interleaving and rate matching operations before the bits are scrambled and mapped to the available resources in the LTE time-frequency grid known as the resource map (introduced in section 2.4.2). Turbo encoding enables error correction to occur at the decoder by concatenating parity bits to the original message bits input into the Coding and Rate Matching block. At the receiver, the turbo decoder iteratively attempts to estimate the receiver codeword by passing probabilities between soft output decoders. The power of turbo decoding is that it enables very long codes with very good results with relatively small implementation complexities [19].

The turbo encoder is built with two rate $1 / 3$ recursive systematic convolutional (RSC) encoders, shown in figure 2.23, with generator matrix $G=\left[1, g_{0} / g_{1}\right]$, where $g_{0}=[1011]$ and $g_{1}=[1101]$ as defined in [18]. The RSC encoders operate on the input bits stream and the interleaved bit stream producing two parity bits for each bit input into the turbo encoder. The concatenated systematic and parity bits produce a fixed rate bit sequence based on the sequence of input bits, known as the mother code. The mother code is then adapted to match the available physical layer resources by puncturing or repeating the mother code to achieve the desired code rate. The amount of physical layer resources (bits) available are determined by the UE's modulation and coding scheme (MCS) selection

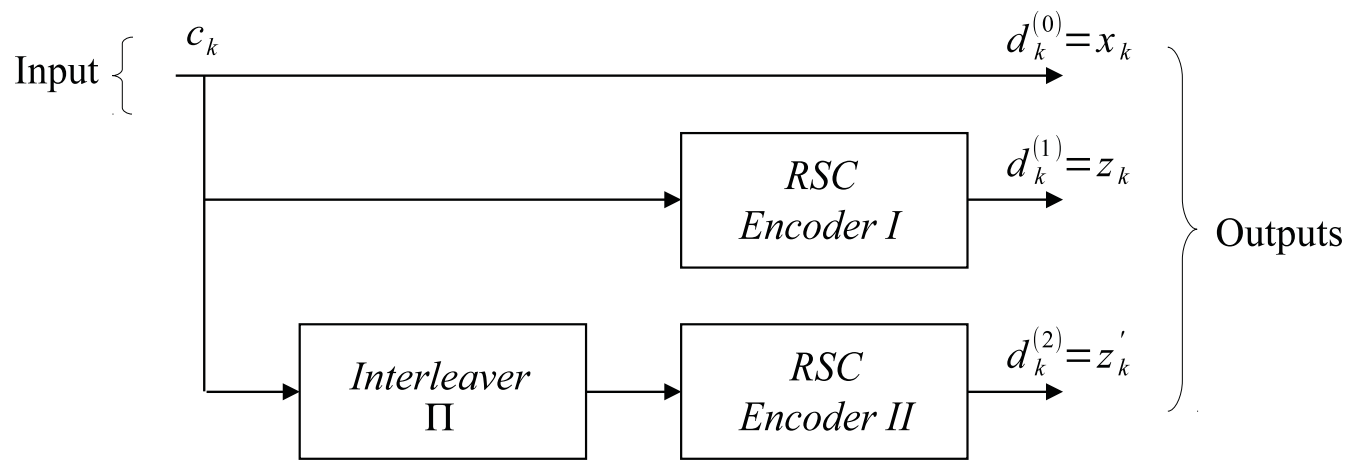

Figure 2.23: Block Diagram of the LTE Turbo Encoder

In LTE, a circular buffer is used to perform rate matching as shown in figure 2.24. The systematic and parity bits of the mother code are interleaved and written to a circular buffer. The bits that will be transmit are read out of the circular buffer serially until the number of bits read out of the circular buffer match the number of data bits available in the 
resource map for transmission. The LTE circular buffer rate matcher arbitrarily selects bits for transmission based on the redundancy version (RV), which sets the starting point in the circular buffer. On the first transmission, the transmitter sends a large number of systematic bits in order to ensure the receiver can decode the message.

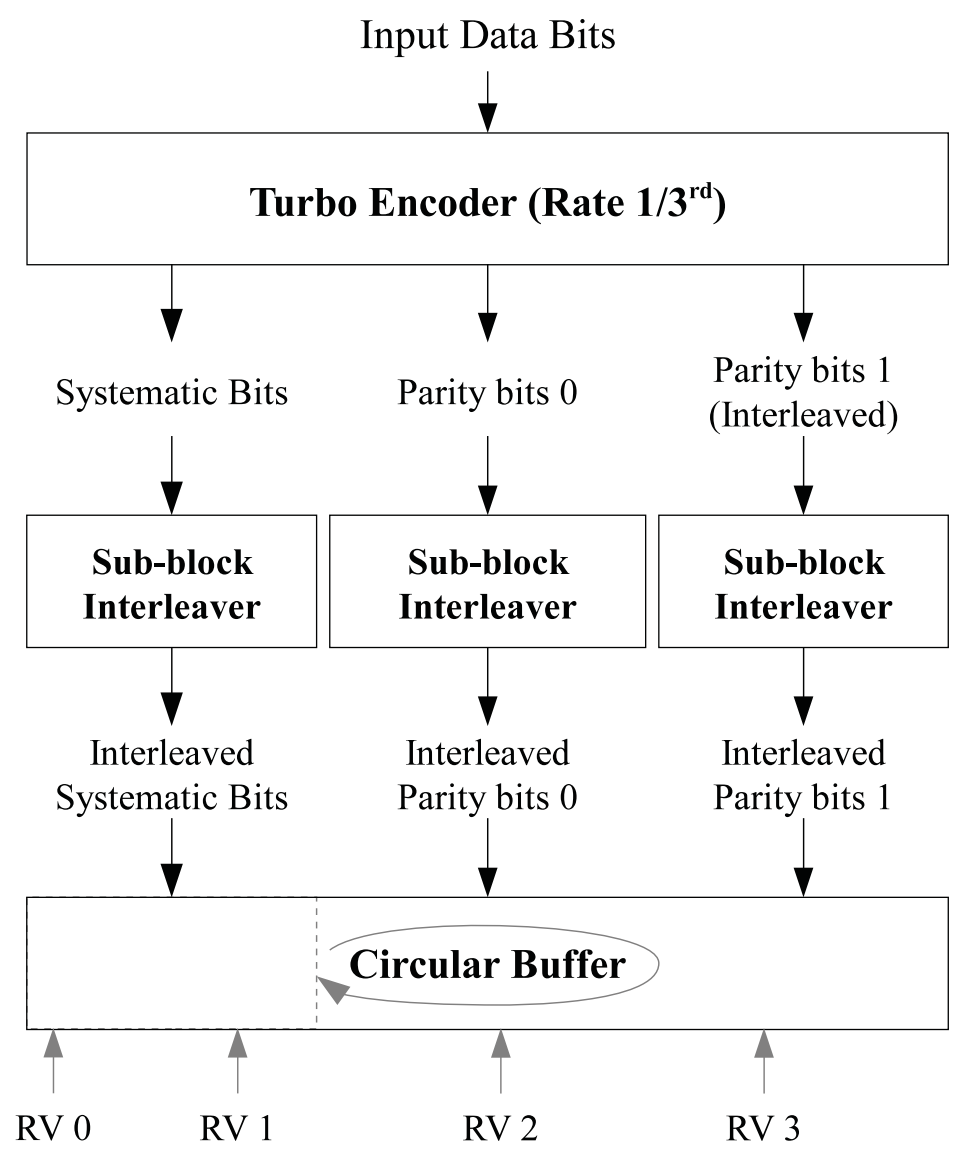

Figure 2.24: Illustration of the LTE Turbo Encoding with the Circular Buffer for Rate Matching

When the UE requests HARQ retransmission of the same packet, the redundancy version (RV) is incremented and a new set of bits from the circular buffer are read out and transmitted. The combination of the circular buffer rate matcher and HARQ in LTE allows the UE to efficiently perform incremental redundancy or chase combining to improve the chances of decoding the codeword after each HARQ transmission.

The bits $b_{0}, b_{1}, \ldots, b_{M_{b i t}-1}$ output from LTE's turbo encoder and rate matching stages are then sent to the Scrambling and Modulation block. 


\section{Scrambling and Modulation}

Scrambling is performed in LTE to randomize the interference between UEs. Length 31 Gold codes are used for this purpose since the sequences generated by Gold codes are not cyclic shifts of each other. When initialized with the following information, the low crosscorrelation between the Gold code sequences reduces possible interference between cells, UEs and codewords.

- Cell ID

- UE ID

- Sub-frame number

- Codeword ID (if more than one codeword is transmit)

The scrambled bits are then mapped to complex modulation symbols $d$ before layer mapping.

\section{Layer Mapping and Precoding}

LTE uses layer mapping to map the codewords to the spatial transmission layers that are available in the channel. A codeword can be mapped to one or more layers, and when an odd number of layers are present, one codeword may be mapped to a single layer while the other is mapped to multiple layers. In release 8 of the 3GPP LTE standards, category 1,2,3 and 4 UEs are limited to two antennas at the receiver. This limits the number of codewords to two, despite the fact that eNodeB's can use more than two antenna elements. This is because LTE was configured as a hybrid scheme; spatial multiplexing is used but the number of codewords are limited to reduce the reporting overhead on the uplink. The redundant antennas are used as a transmit diversity scheme, where multiple codewords are transmit on twice as many antennas as necessary providing diversity or beamforming gains when precoding is applied.

When a single layer and single codeword is being processed for transmission, the modulated symbols $d^{(q)}(i)$ are directly mapped via $x^{(0)}(i)=d^{(0)}(i)$ to complex layer symbols $x^{(v)}(i)$ according to the procedures in section 6.3.3.1 of [1]. If multiple antennas are present in the system the single layer is mapped to all antennas and the system exhibits diversity gain.

If more than one layer and codeword are being processed, layer mapping and precoding are performed before symbol-to-resource element mapping occurs according to section 6.3.3 of [1]. For spatial multiplexing, layer mapping is performed according to table 
6.3.3.2-1 in [1]. In release 8 of the 3GPP standards, 1 or 2 codewords could be mapped to up to 4 layers. The number of layers is extended to 8 in release 11 of [1].

When using transmit diversity, layer mapping is relatively simple since transmit diversity transmits a single codeword. As described in table 6.3.3.3-1 of [1], transmit diversity maps its only codeword to $v$ layers before precoding and transmission.

Precoding enables beamforming to take place to increase the performance of the LTE system. During a single layer beamforming transmission each antenna port transmits the same information from all antennas but the symbols emit from the antennas are weighted to direct the electric field toward the receiver and maximize the received signal power. This is known as beamforming and is used in LTE transmission modes such as closed-loop rank1 beamforming TM6 (section 2.5.5) and TM4 (section 2.5.4) when transmitting a single codewords.

When multiple codewords are permitted spatial multiplexing modes use transmit and receive beamforming to maximize the rate of transmission of all the streams available between the transmitting and receiving antennas. The precoder is selected by the UE and reported to the BS in the form of a precoding matrix indicator (PMI) which points to a particular matrix in the codebook defined in the 3GPP LTE standard [1]. The application of the UE selected precoder to the multi-stream OFDM transmissions greatly increases the transmission rate over the MIMO channel.

\section{Symbol to Sub-carrier Mapping}

The process of mapping the complex modulated symbols $d$ to the sub-carriers is known as resource element mapping. The sub-carriers are called resource elements (REs) and blocks of REs are allocated at a time by the base station scheduler to the UEs. The resources available are allocated and discussed in units of physical resource blocks (PRBs) and subframes in the resource map.

The resource map is a representation of the OFDM resources at the physical layer in the time and frequency domain. The resource map is a grid of REs representing sub-carriers with dimensions in the time and frequency domain, where the time domain is represented by OFDM symbols and the frequency domain is in sub-carriers. A physical resource block (PRB) is the minimum unit of allocation in LTE and has the same time duration as a slot $(0.5 \mathrm{msec})$. A physical resource block is 12 sub-carriers by 7 OFDM symbols wide when using the normal cyclic prefix or 6 OFDM symbols wide when using the extended cyclic prefix. LTE also defines frames and sub-frames which have a duration of $10 \mathrm{msec}$ and 1 
msec each as shown in figure 2.26. The resource map and sub-frame are discussed further in section 2.4.2.

The resource element mapping block begins by reserving particular sub-carriers in the resource map for reference signals and control channels such as the PDCCH. if they are to be transmit in the current OFDM symbol. The remaining available REs are used by the PDSCH to transmit data to the UE. The precoded layer symbols are mapped to the available PDSCH REs on the available antenna ports according to the transmission scheme selected just before OFDM symbol generation and $\mathrm{CP}$ addition.

\section{OFDM Symbol Generation and Cyclic Prefix}

Finally, the last stage in the transmission chain, before up-conversion to RF, is the IFFT and CP addition. The IFFT step generates time domain samples from the input resourcemapped frequency domain symbols. Once the time domain symbols are available, samples from the end of the time domain sequence $x[n]$ are copied and pre-pended to the front of the time domain sequence giving the sequence its cyclical property.

\section{RF Up-Conversion and Power Amplification}

Transmission is accomplished by up-converting the baseband signal to an available carrier frequency in the operator's spectrum resources. The baseband signal is generated by the digital to analog conversion (D/A) stage and the complex signal is up-converted to RF via I-Q modulation.

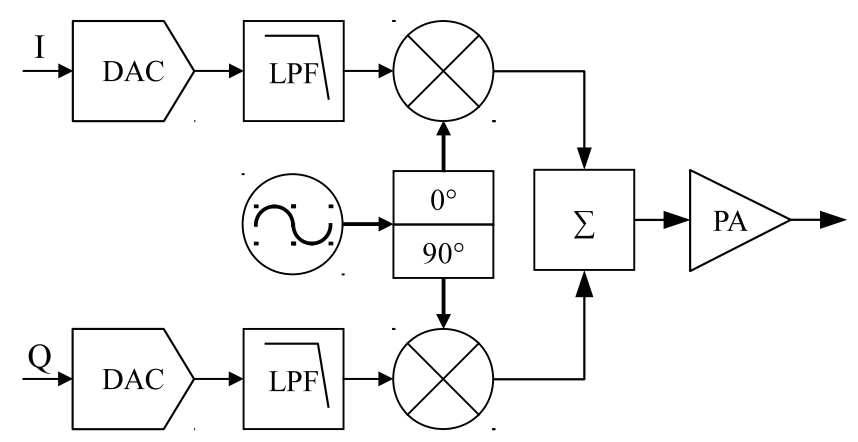

Figure 2.25: IQ Modulator and RF Up-Conversion

This stage is not included in wireless simulation tools since it is not necessary for simulation purposes and the complexity and simulation time would increase dramatically. 


\subsubsection{LTE Downlink Radio Frame}

The 3GPP wireless standard defines the LTE radio frame as 10 continuous sub-frames, where a sub-frame is 14 or 12 OFDM symbols wide in the time domain, for a system employing a normal $\mathrm{CP}$ and a system employing an extended $\mathrm{CP}$ respectively. The subframes are further split into 2 slots containing 7 OFDM symbols when using the normal CP or 6 OFDM symbols when the extended $\mathrm{CP}$ is used to overcome channels exhibiting long delay spread.

The LTE frame structure is shown in figure 2.26. The frame consists of 10 sub-frames, which are split into 2 slots each. The sub-frame is defined as the LTE transmission time interval (TTI).

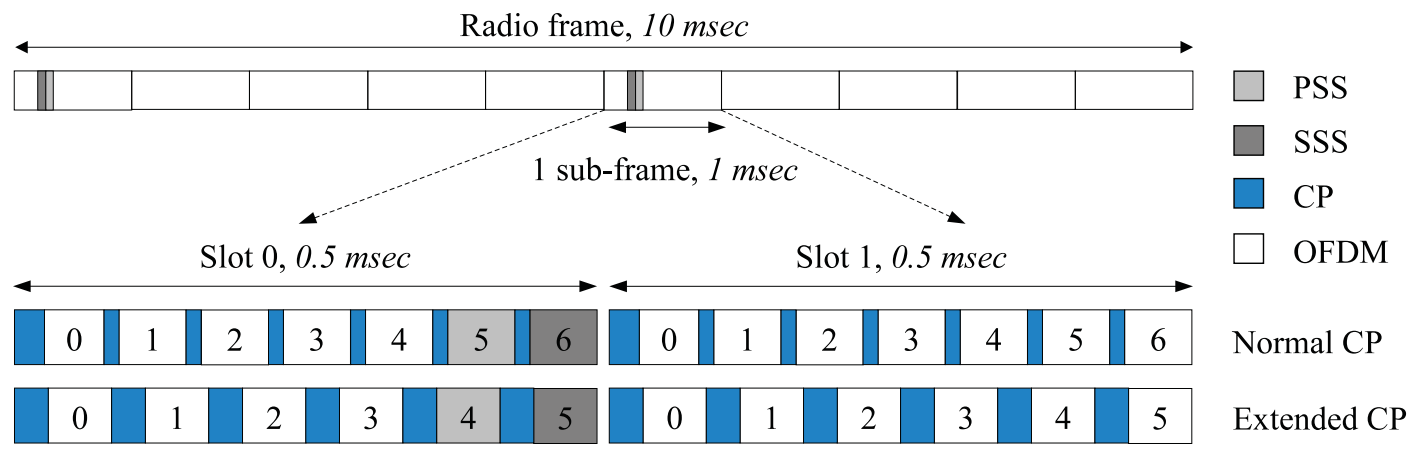

Figure 2.26: Decomposition of the LTE Frame into Sub-frames and Slots

The LTE physical downlink shared channel (PDSCH) is a link between the eNodeB and the UE and is based on the resource map grid. The resource map dimensions are denoted as $l$ OFDM symbols in the time domain and $k$ sub-carriers in the frequency domain as shown in the grid in figure 2.27. The allocated spectrum is split into physical resource blocks (PRBs), which are 12 sub-carriers wide $(180 \mathrm{kHz})$ in the frequency domain and one slot (0.5 msec) wide (6 or 7 OFDM symbols) in the time domain.

\subsubsection{Synchronization Signals}

Time and frequency synchronization is required to align the receiver with the transmit OFDM symbols in the time domain and with the sub-carrier peaks in the frequency domain. In the time domain the receiver needs to match the base station timing and the 
One downlink slot $T_{\text {slot }}$
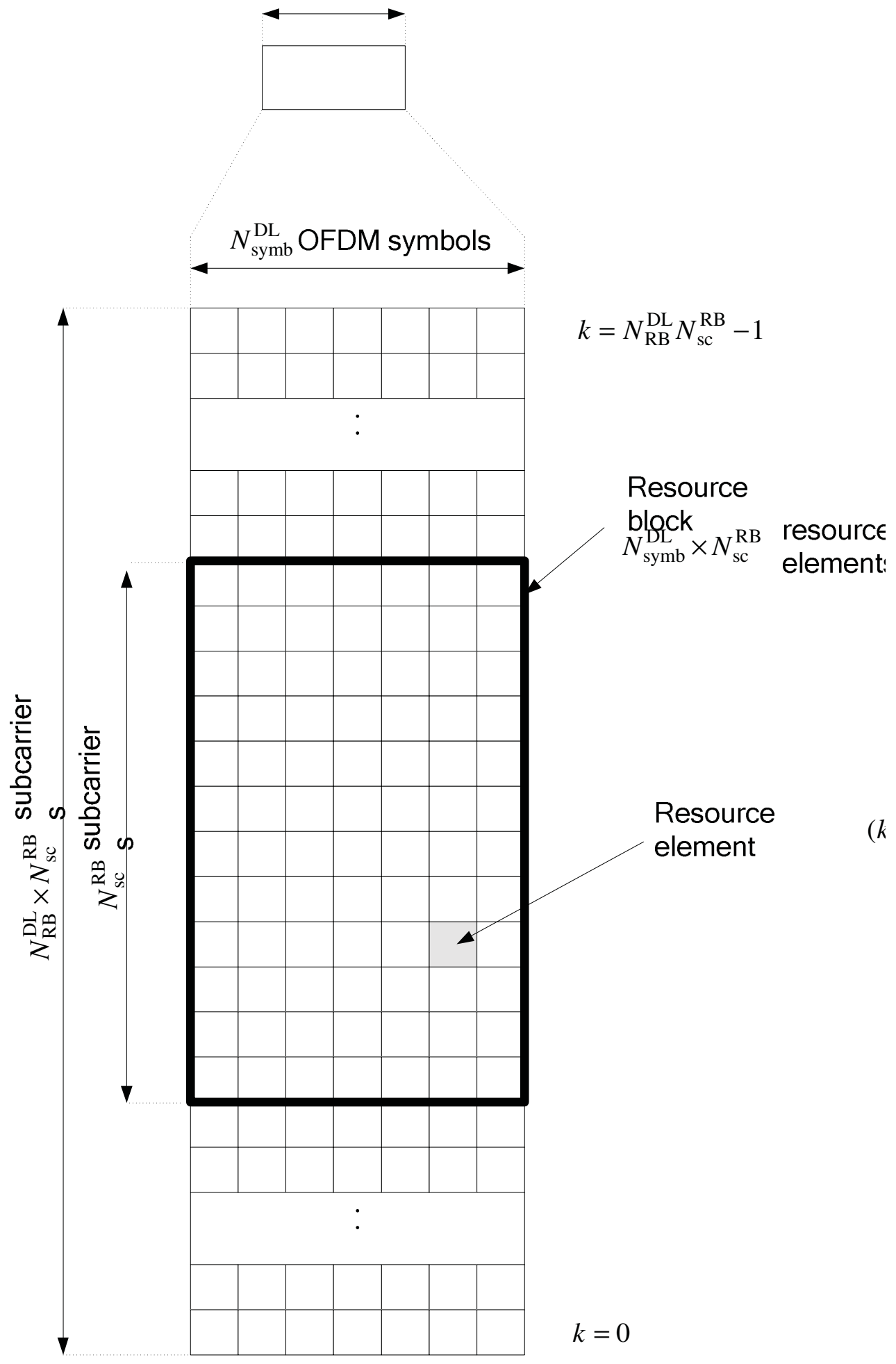

Figure 2.27: LTE Resource Map From Figure 6.2.1-1 of [1] 
propagation delay introduced by the channel. The transmit signal is also affected by carrier frequency offsets (CFO) in the frequency domain due to Doppler shift and oscillator mis-match. The carrier frequency offset due to mobility and oscillator mis-match and time domain synchronization must be compensated for by the UE before decoding the downlink (PDSCH) transmissions.

In order to correct the frequency offset and timing mis-match, two LTE downlink subframes are embedded with primary synchronization signal (PSS) and secondary synchronization signal (SSS). The PSS and the SSS are transmit by the eNodeB in the first slot of sub-frame 0 and 5 as shown in 2.28. The PSS and SSS are used to perform frame, sub-frame and slot synchronization and determine physical layer IDs and transmission characteristics.

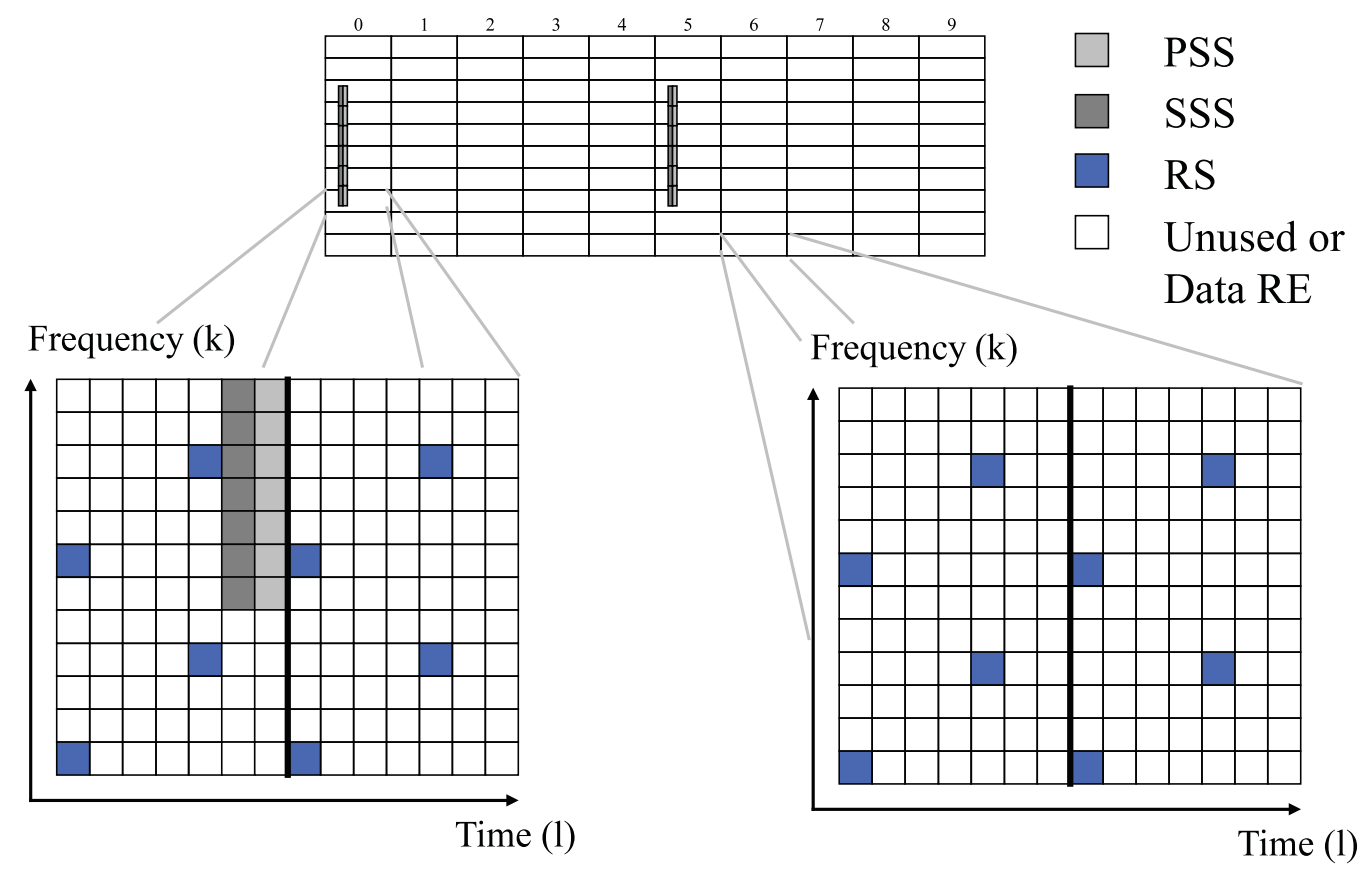

Figure 2.28: Resource Map Showing PSS, SSS and RS

The benefit of transmitting the synchronization signals twice per frame is that it enables the UE to quickly acquire the cell or perform cell re-selection since acquiring the PSS and SSS is required before measuring the SNR of the reference signals. The PSS and SSS are transmit in the central 6 PRBs surrounding the direct current (DC) sub-carrier in the frequency domain. Of the 72 sub-carriers in the central 6 PRBs, only 62 sub-carriers surrounding the DC sub-carrier are used. The reason for using 62 sub-carriers centered about the DC sub-carrier for synchronization is for power saving, low processing complexity and 
simplicity. The UE can perform synchronization with a short 64-point FFT without knowledge of the cell since the synchronization signals are invariant in position and in size in the frequency domain.

\section{Primary Synchronization Signals}

The PSS are located in the last OFDM symbol of the first slot of sub-frame 0 and 5 for frame structure type I. Cell search begins with a search for the PSS. The PSS are acquired using non-coherent detection since the channel is not yet known. The eNodeB transmits the PSS using constant amplitude zero autocorrelation (CAZAC) sequences, known as Zadoff$\mathrm{Chu}(\mathrm{ZC})$ sequences, which permit slot timing detection and the acquisition of the physical layer ID which may take on 3 possible identities.

\section{Secondary Synchronization Signals}

After the acquisition of the PSS the channel is known and the search continues with the acquisition of the SSS using coherent detection. The SSS are maximum length sequence (Msequences) which are located in the before last OFDM symbol of the first slot of sub-frame 0 and 5 for frame structure type I transmissions (frequency-division multiplexing (FDD)). The M-sequences are length-31 binary sequences with low cross-correlation properties that are cyclically shifted based on the cell group identity $N_{I D}^{(2)}$. The SSS transmissions are intentionally different to allow the receiver to perform radio frame synchronization which is not possible with the PSS since the PSS transmissions are identical.

The SSS are positioned in four possible locations relative to the PSS. The four different SSS positions allow the UE to determine the length of the cyclic prefix (CP) used in the transmissions and the duplexing scheme (FDD or time-division duplexing (TDD)). The SSS are also encoded with a cell ID where 168 group identities are possible.

\subsubsection{Reference Signals and Antenna Ports}

In order to remove the channel effects, transmission systems use training sequences or known signals embedded in the transmissions to allow the receiver to estimate the channel. In LTE, known sub-carriers called reference signals (RS) are inserted into the resource map enabling the receiver to estimate the channel and correct channel effects such as phase rotation and frequency domain amplitude variations. 
By strategically placing reference signals in the downlink resource map at spacings in the time and frequency domain that satisfy the sampling theorem, the channel's time and frequency domain characteristics can be measured and the channel can be estimated. The receiver uses the acquired channel estimates at the reference signal sub-carriers and the characteristics of the channel impulse response to reproduce a complete channel estimate for use at the channel equalization step discussed in section 2.4.5. Channel equalization is then performed on the sub-carriers to counter the effects introduced by the channel before the receiver attempts to decode the received data.

\section{Types of References Signals}

There are 3 types of reference signals transmitted on the downlink in LTE release 8, namely the cell-specific reference signal (CRS), the UE-specific reference signal (UE-Spec RS) and the multimedia broadcast single frequency network reference signal (MBSFN-RS). The purpose of each is described in table 2.6.

Table 2.6: Types of Downlink Reference Signals Defined in 3GPP LTE Rel 8

\begin{tabular}{ccl}
\hline RS & $\begin{array}{c}\text { Antenna } \\
\text { Ports }\end{array}$ & \multicolumn{1}{c}{ Description } \\
\hline \hline CRS & Port 0-3 & $\begin{array}{l}\text { Cell-specific } R S \text { patterns are transmit across the entire } \\
\text { system bandwidth based on the antenna port allocation } \\
\text { to allow the UE to acquire the BS, measure the channel } \\
\text { for link adaptation and frequency selective scheduling } \\
\text { (FSS). }\end{array}$ \\
UE-Spec RS & Port 5 & $\begin{array}{l}\text { UE-specific } R S \text { are used in single layer beamforming } \\
\text { (transmission mode } 7 \text { (TM7)) where the RS are pre- } \\
\text { coded with the same precoder as the data to allow the } \\
\text { UE to perform coherent demodulation. } \\
\text { Multi-media single frequency network } R S \text { are used in } \\
\text { an single frequency network which broadcasts com- } \\
\text { mon content to multiple users simultaneously. Multi- } \\
\text { ple eNodeBs may participate in the broadcast network } \\
\text { and all data and RS in a particular multimedia broad- } \\
\text { cast single frequency network (MBSFN) are the same } \\
\text { and carefully synchronized. }\end{array}$ \\
\hline
\end{tabular}




\section{Cell-Specific Reference Signals}

The most commonly used reference signals are the cell-specific RS (CRS) because they are required to acquire and connect to base stations and are therefore always broadcast across the frequency domain bandwidth in the downlink portion of the spectrum.

The primary uses for the cell-specific RS are:

- Assess the quality of the signal received from nearby base stations for cell selection.

- The CRS are used to perform channel estimation and equalization for decoding purposes.

- The CRS are used to assess the wideband or sub-band channel quality and select a modulation \& coding scheme (MCS) as well as a precoder (PMI) and rank for the next set of transmissions.

\section{Antenna Ports}

When multiple antennas are used, there may be more than one set of cell-specific RS used for transmission. One or more sets of antennas that are assigned a particular cell-specific RS pattern is called an "antenna port". The antenna ports are a key component of LTE since they enable MIMO transmissions that permit the high bit rates set forth by ITU-R in the International Mobile Telecommunications (IMT) requirements.

The antenna port reference signals are used to perform channel estimation and equalization, adaptive modulation and coding, and rank and precoder selection when a spatial multiplexing transmission mode is used. The base station can be configured to transmit a unique reference signal pattern on each antenna element or repeat the same pattern on more than one antenna to achieve diversity gain. Therefore a limit is imposed on the number of antenna ports based on the available antenna elements. The number of antenna ports used for transmission is always less than or equal to the number of antennas available.

The following antenna port configurations and corresponding RS patterns are used to achieve the simulation results presented in this thesis:

- For 2 transmitters \& 2 antenna ports, the RS patterns in figure 2.29 are used.

- For 2 transmitters \& 2 antenna ports, the RS patterns in figure 2.30 are used.

- For 4 transmitters \& 4 antenna ports, the RS patterns in figure 2.31 are used. 


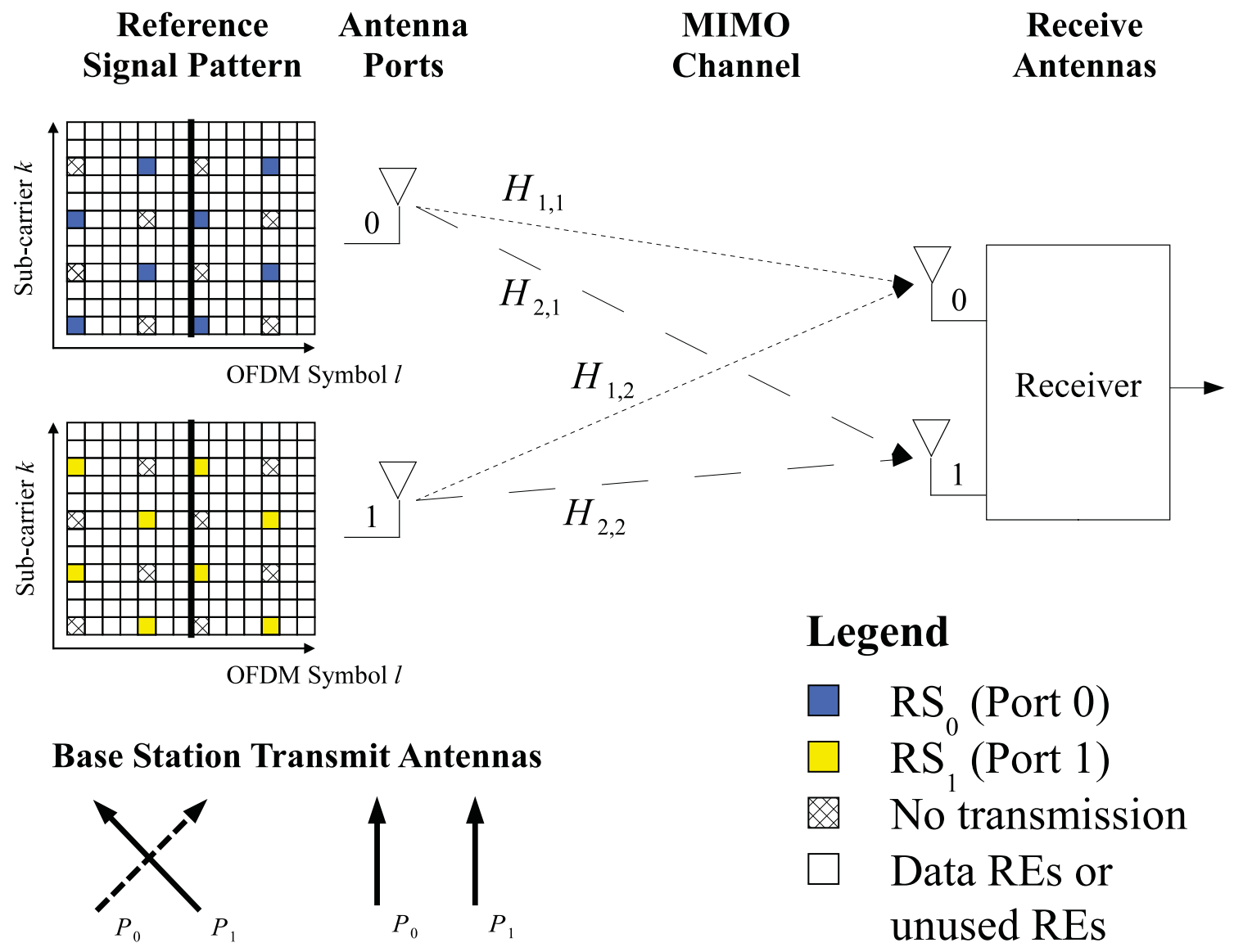

Figure 2.29: Cell-Specific Reference Signal Patterns for 2-Tx 2-Ports 


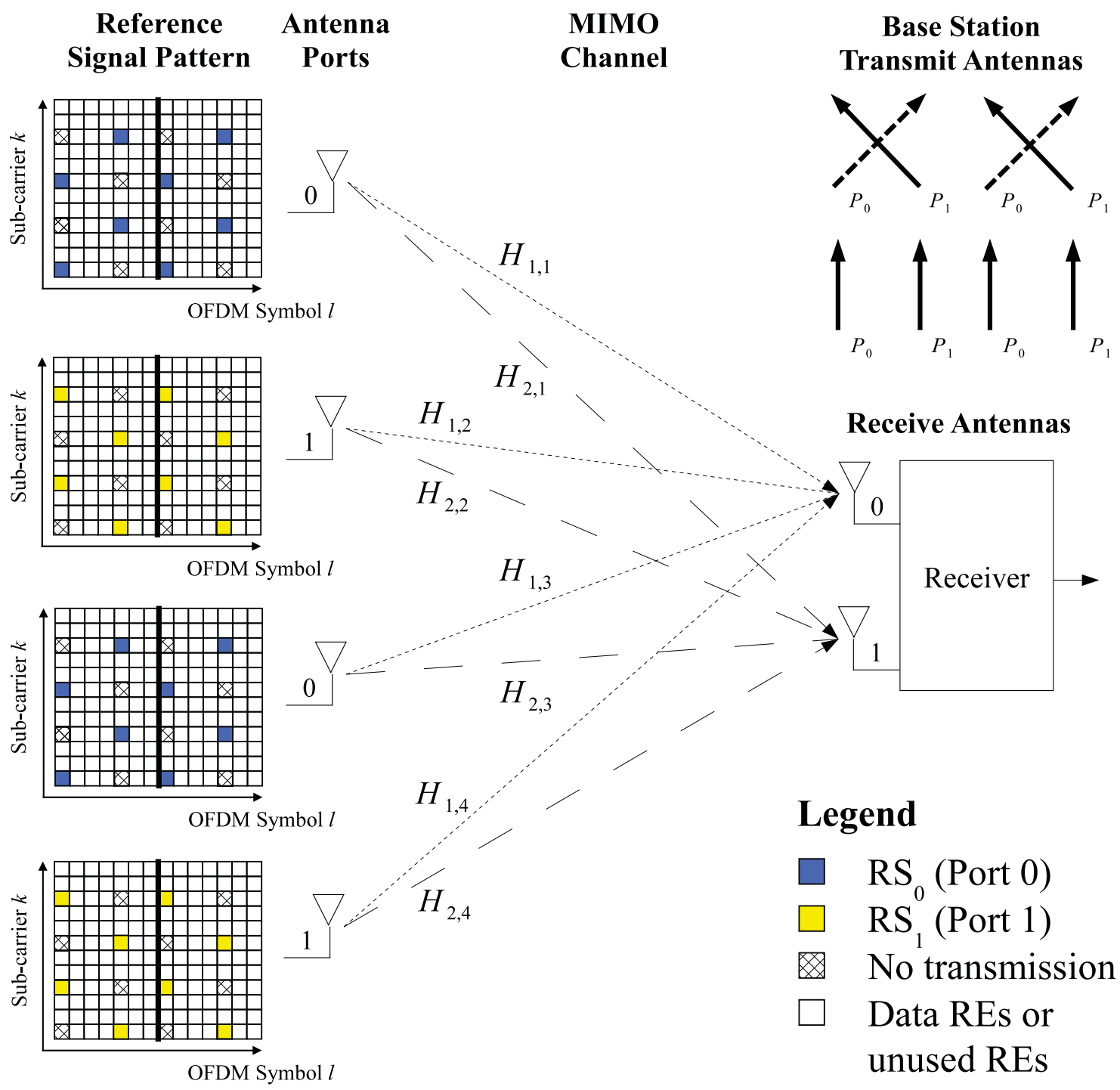

Figure 2.30: Cell-Specific Reference Signal Patterns for 4-Tx 2-Ports

The RS patterns are used at the UE to build the channel estimate for a particular BS antenna port. From the point of view of the UE, the individual channel estimates determined from the antenna ports can be from separate antenna elements or composite channels from multiple antenna elements. Composite channels are the resulting channel seen by the UE when an antenna port is assigned and transmit from more than one antenna element at a time. The purpose of the antenna port is to enable the UE to build the wireless channel's MIMO channel matrix and to determine the channel's rank. A rank $>1$ can be used to send 


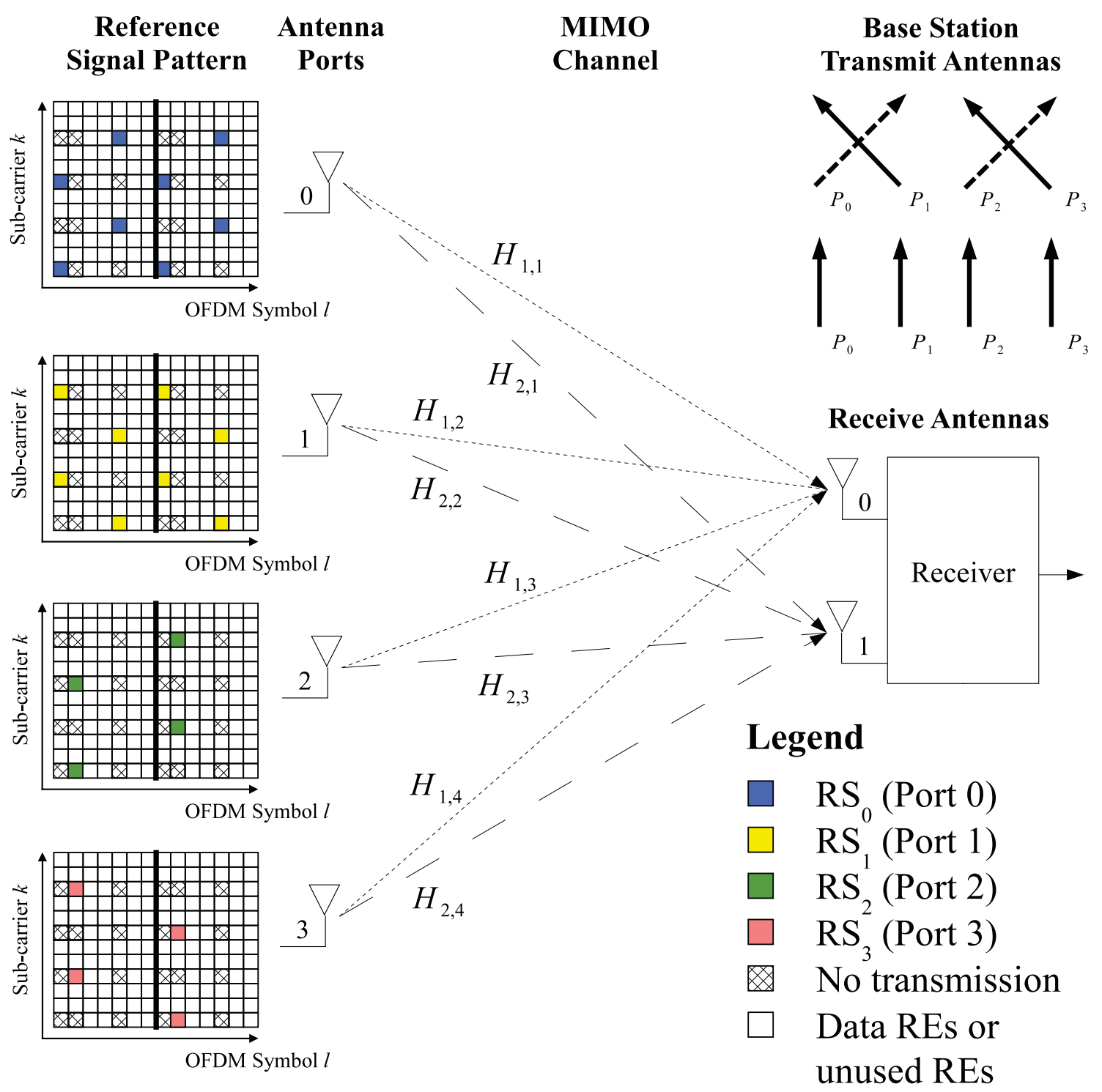

Figure 2.31: Cell-Specific Reference Signal Patterns for 4-Tx 4-Ports 
multiple codewords without needing to increase the system's transmit power or bandwidth.

In this thesis, two UE receive antennas will be used along with 4 BS transmitters. The BS antenna configurations discussed in this section along with various element polarizations and correlations will be used to evaluate the performance of the downlink with various antenna configurations. The specific BS antenna configurations used for simulations are further discussed in section 4.1.1 of chapter 4 .

\subsubsection{Channel Estimation}

To estimate the channel for channel analysis and equalization, pilot aided channel estimation (PACE) is used. The UE uses the strategically positioned reference signals, discussed in section 2.4.4, to estimate the channel and remove its impact on the received sub-carriers.

The received sub-carrier is modeled by equation 2.47 where $\mathbf{Y}=\left(Y_{0}, Y_{1}, \ldots, Y_{L-1}\right)$ is the received symbol after $\mathrm{CP}$ removal and FFT at each sub-carrier, $\mathbf{H}=\operatorname{diag}\left(H_{0}, H_{1}, \ldots, H_{L-1}\right)$ is the channel matrix, $\mathbf{X}=\left(X 0, X_{1}, \ldots, X_{L-1}\right)$ is a vector of the transmit symbols on each subcarrier before IFFT, and $\mathbf{N}=\left(N_{0}, N_{1}, \ldots, N_{L-1}\right)$ is a random variable representing frequency domain complex circular noise added by the channel.

$$
Y_{k, l}=H_{k, l} X_{k, l}+N_{k, l}
$$

The chosen LTE "diamond" RS pattern was carefully selected since it is a balance between frequency domain and time domain support when compared to other RS patterns. The RS patterns are a trade-off between optimal spacings in the time and frequency domain. The RS spacing chosen in the time and frequency domain affect the UE's ability to reconstruct the complete channel estimate accurately. The RS spacing is also a delicate balance between spectral efficiency and support for high delay spread channels (ie. frequency selective channels) seen in MBSFN or terrestrial scenarios and high channel Doppler (ie. high UE velocity).

The RS pattern and spacing selected by the 3GPP had to be carefully selected to allow the receiver to overcome the channel and meet the IMT performance requirements and goals. Work done by several authors, such as the authors in [20], demonstrate the strengths and weaknesses of each pattern under various scenarios. The comb pattern, for example, can operate well in a frequency selective environment if the UE mobility is low. However, the diamond pattern shown in figure 2.32 was standardized since it provided low overhead and good channel reconstruction in both the frequency domain and the time domain in 
low mobility and a frequency flat channel scenario. The diamond pattern continues to be effective if a single channel effect starts to increases because low channel impairment in the other domain will allow the channel reconstruction filter to adequately interpolate the offset channel estimates.

Frequency (k)
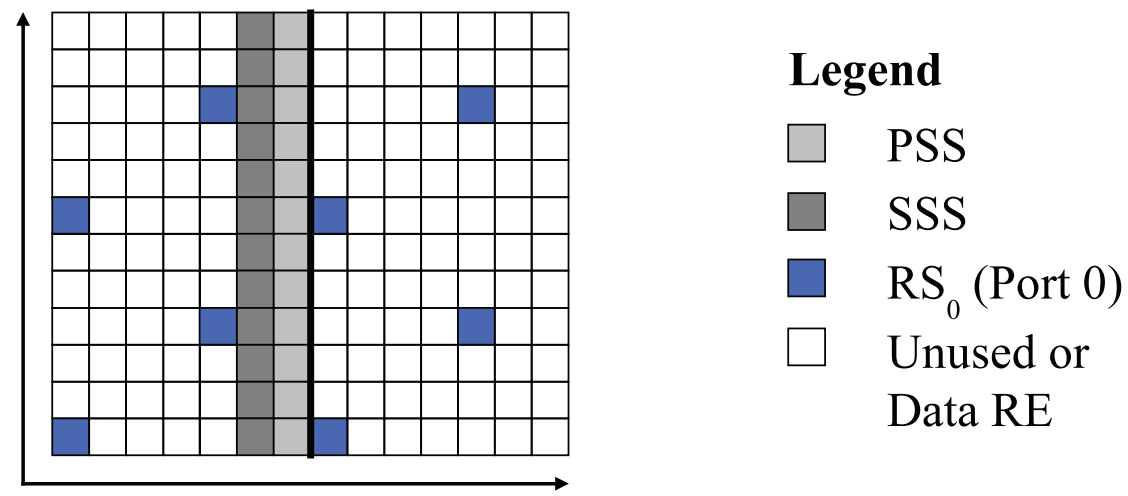

Time (1)

Figure 2.32: Downlink Cell-Specific Reference Signal Pattern $\left(R S_{0}\right.$ for Port 0$)$

The two principal maximum likelihood (ML) channel matrix estimators are the least squares (LS) estimator and the minimum mean square error (MMSE) estimator. Both estimators try to minimize the error between the channel estimate $\hat{H}_{p, l}$ and the actual channel $H_{p, l}$ at the pilot location. The received symbol $Y_{p, l}$ at a known sub-carrier is given by equation 2.48, where $N_{p, l}$ is the noise, $X_{p, l}$ is the transmit symbol and $H_{p, l}$ is the channel at reference symbol $p$ of OFDM symbol $l$.

$$
Y_{p, l}=H_{p, l} X_{p, l}+N_{p, l}
$$

Using the LS method for simplicity, $\hat{H}$ in equation 2.49 is the estimate of the channel at the RS at sub-carrier $(p, l)$ where $p$ is the location of the known RS and $l$ is the OFDM symbol.

$$
\hat{H}_{p, l}=Y_{p, l} X_{p, l}^{-1}
$$




\section{Time Domain RS Spacing}

During the LTE standardization process, the LTE downlink was designed to support UE velocities up to $500 \mathrm{~km} / \mathrm{hr}$ for high speed train scenarios. Based on this requirement the optimal RS placement in the time domain could be estimated using equation 2.50 and the Nyquist sampling theorem since the channel has been shown to change proportionally to the UE velocity.

$$
f_{m}=f_{c} \cdot v / c
$$

Using the UE velocity $v$ and maximum Doppler frequency $f_{m}$ relationship in equation 2.50, along with a downlink carrier frequency $f_{c}$ of $2.6 \mathrm{GHz}$, a required velocity $v$ of 500 $\mathrm{km} / \mathrm{hr}$ and $c$ the speed of light, the maximum channel Doppler frequency that is required to be supported at $2.6 \mathrm{GHz}$ is $1204 \mathrm{~Hz}$ as shown in 2.51 .

$$
f_{m}=\frac{2.6 \mathrm{GHz} \cdot(500 \mathrm{~km} / \mathrm{hr} \cdot 1 / 3.6)}{3 \cdot 10^{8} \mathrm{~m} / \mathrm{s}} \approx 1204 \mathrm{~Hz}
$$

In order to sample and faithfully track the channel in the time domain, reference signals are placed at intervals of $1 /\left(2 f_{m}\right)$ seconds in order to satisfy the Nyquist sampling theorem as shown in 2.52 .

$$
T_{c}=\frac{1}{2 f_{m}}=\frac{1}{2 \cdot 1204 \mathrm{~Hz}} \approx 0.415 \mathrm{msec}
$$

The actual placement of the reference signals in the time domain in LTE are every 7-th symbol ( 1 per slot), corresponding to a sampling interval of $7 \cdot T_{s} \approx 0.5 \mathrm{msec}$. However, using the diamond pattern and under the assumption that delay spread is low enough, the offset reference signals in other OFDM symbols may be sufficiently correlated to be used as additional sample points to track the time-varying channel. For example, reference signals at sub-carrier $Y_{k, l}=Y_{3,4}$ provides useful channel estimate information about the channel at sub-carrier $Y_{0,3}$ in order to better follow time variations.

Similarly, the reference signals at sub-carrier $Y_{3,4}$ can be used at sub-carrier $Y_{3,0}$ to support frequency domain channel estimation in OFDM symbol 0 , if the channel is slowly varying in time and the correlation between OFDM symbol 3 and OFDM symbol 0 is high enough. 


\section{Frequency (k)}

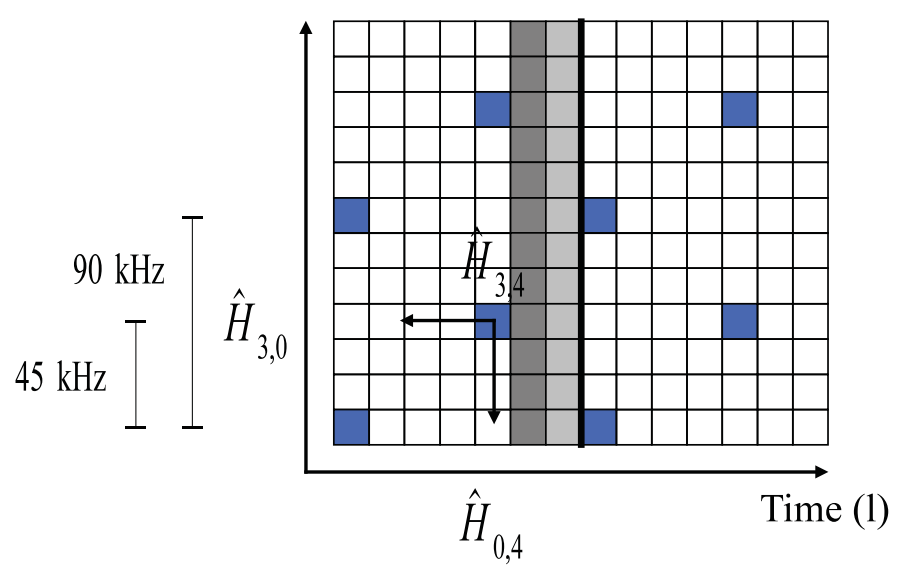

Figure 2.33: Downlink Reference Signal Correlation in Low Delay Spread or Low Mobility Scenarios

\section{Frequency Domain RS Spacing}

The process of determining the frequency domain reference signal spacing is similar in principle to the process of determining the time domain RS spacing, but the frequency domain RS spacing is dependent on the expected frequency selectivity instead of the rate of change of the channel in the time domain. Therefore, in order to find the required RS spacing in the frequency domain, the coherence bandwidth is used to determine the required sampling interval.

The coherence bandwidth $B_{c}$ is a measure of the correlation between two points in the frequency domain representation of the channel. From [8], the definition of the 50\% and $90 \%$ coherence bandwidths of a channel are shown to be related to the inverse of the delay spread $\sigma_{\tau}$ and can be used to estimate the required spacing for typical delays spread values obtained from wireless channel measurements. Equation 2.53 imposes a higher correlation value leading to a more conservative coherence bandwidth estimate. Meanwhile, the 50\% coherence bandwidth produced by equation 2.54 is a looser definition leading to wider coherence bandwidths.

$$
\begin{aligned}
B_{c, 90 \%} & =\frac{1}{50 \sigma_{\tau}} \\
B_{c, 50 \%} & =\frac{1}{5 \sigma_{\tau}}
\end{aligned}
$$

The delay spread of three widely used 3GPP conformance testing channel models (EPA, 
EVA and ETU) are shown in table 2.7 and the corresponding 50\% and 90\% coherence bandwidths are listed to show how closely the reference signals must be spaced based in order to be able to adequately reproduce the channel amplitude variations in the frequency domain.

Table 2.7: Coherence Bandwidth for 3GPP Conformance Testing Channel Models

\begin{tabular}{cccc}
\hline Channel Model & Delay Spread $E\left(\sigma_{D S}\right)$ & $B_{c, 90 \%}$ & $B_{c, 50 \%}$ \\
\hline \hline Extended pedestrian A (EPA) & $43 \mathrm{nsec}$ & $463.7 \mathrm{kHz}$ & $4637 \mathrm{kHz}$ \\
Extended vehicular A (EVA) & $357 \mathrm{nsec}$ & $56.1 \mathrm{kHz}$ & $560.8 \mathrm{kHz}$ \\
Extended typical urban (ETU) & $991 \mathrm{nsec}$ & $20.2 \mathrm{kHz}$ & $201.8 \mathrm{kHz}$ \\
\hline
\end{tabular}

A reference signal spacing of 6 sub-carriers wide in the frequency domain is equivalent to $90 \mathrm{kHz}$. A reference signal spacing of 6 sub-carriers can support all the channels listed in table 2.7 using the 50\% coherence bandwidth metric and the EPA channel using the more stringent $90 \%$ coherence bandwidth metric.

When the UE velocity is reasonably low, interpolation methods such as linear interpolation, spline cubic fitting or low pass filtering can make use of the nearby reference signals and channel statistics to better reconstruct the complete channel by selecting a more appropriate reconstruction filter or interpolation weight. For instance, at sufficiently low UE velocities the estimate at $\hat{H}_{3,4}$, in figure 2.33 , can be used to improve the channel estimate at $\hat{H}_{3,0}$ due to high correlation between the two sub-carriers. The interpolated information from $\hat{H}_{3,4}$ at $\hat{H}_{3,0}$ effectively doubles the number of samples in the first OFDM symbol of the sub-frame. The same interpolation also increases the number of frequency domain samples in the other OFDM symbols with reference signals and the overall frequency domain spacing shrinks from 6 sub-carriers to 3 sub-carriers. The use of the offset reference signals from other OFDM symbols improves the system's ability to reproduce a frequency selective channel. The supported coherence bandwidth of the LTE diamond pattern becomes approximately $45 \mathrm{kHz}$, which is sufficient to support frequency domain reconstruction in all the 3GPP conformance testing channel models in table 2.7, except the ETU channel model when the $90 \%$ coherence bandwidth metric is used. 


\section{Channel Reconstruction}

The complete channel estimate across a sub-frame is obtained by low pass filtering in each domain (time and frequency) as shown in figure 2.34. The full 2D channel estimate obtained with 2D filtering is optimal in terms of the channel estimate mean square error (MSE) but it was shown to be separable into two 1D filtering passes in [21]. The two 1D filters results in a complete sub-frame channel estimate that is nearly as good as the subframe channel estimate that is achieved using the optimal 2D filter but the 1D reconstruction filters are easier to implement.

Frequency (k)

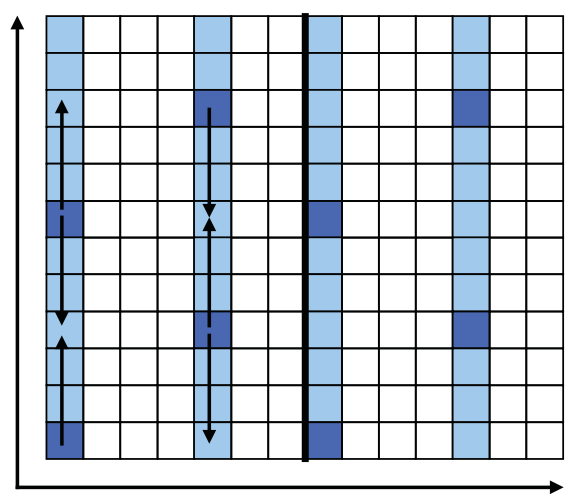

Time (l)
Frequency (k)

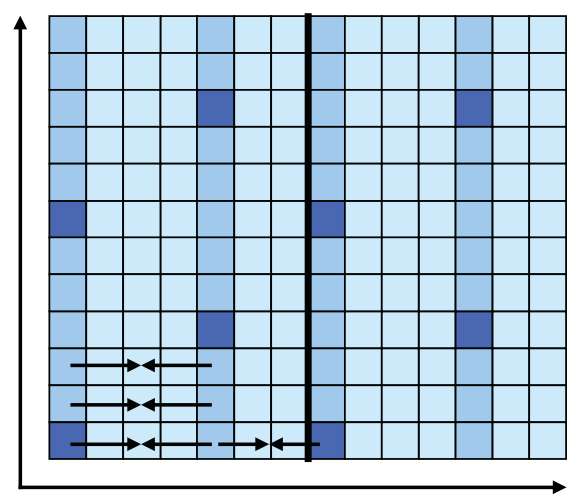

Time (l)

(a) Frequency Domain Filtering Pass For Channel Estimation

(b) Time Domain Filtering Pass For Channel Estimation

Figure 2.34: 2 x 1D Channel Estimate Interpolation

The reference signal pattern that is standardized in [1] is a low overhead pattern that is sufficient for channel estimation in the time domain at low velocity and is capable of reproducing channels amplitudes variations in the frequency domain for most of the 3GPP conformance testing models without significant loss. As Doppler increases due to increasing mobility and as the delay spread $\sigma_{\tau}$ increases, the channel estimation and system performance degrades gradually. The choice of the RS pattern is a careful trade-off between channel estimation performance, overhead, throughput and support for very high UE velocities, typical channel delay spreads and the capability to perform multi-layer transmissions. 


\subsubsection{LTE Link Adaptation}

In order to achieve good spectral efficiency, the downlink transmissions are adapted to the current channel conditions in order to maximize the amount of information that can be transmit across the channel. In LTE the control loop that monitors the channel and adapts the downlink transmissions is known as "link adaptation". Unlike some previous generation wireless communication technologies such as code division multiple access (CDMA), the transmit power is kept constant in LTE while the transmission modulation and coding scheme (MCS) is varied to make the best use of the channel.

\section{Channel Measurements for Link Adaptation}

A condition for the use of adaptive modulation and coding (AMC) is that knowledge about the channel is required at the base station, in order to adapt the modulation and coding scheme to the channel. LTE uses known reference signals, such as the cell-specific reference signals (CRS), to enable the UE to estimate the downlink channel. The CRS are strategically placed in the downlink sub-frames and transmit by the base station as described in section 2.4.4. The UE then estimates the channel at the reference signals and reconstructs the complete channel for each antenna port, as described in section 2.4.5.

The complete channel estimate is used to determine the optimal transmission scheme for the next downlink transmission. The CRS are used to acquire the SNR of the serving cell which is used to determine the new modulation and coding scheme for future transmissions. The rank is then found by analyzing the MIMO channel matrix using methods such as SVD. The number of transmission layers and codewords is determined by the analysis of the channel. Finally, if closed-loop spatial multiplexing transmissions are enabled by the operator, the precoder that maximizes the capacity of the measured channel is found by exhaustive search.

A UE may be configured to report the measured channel state information to the base station on a wideband or sub-band basis for each codeword. The wideband SNR measurement calculates the average SNR for the entire downlink bandwidth using the effective exponential SNR mapping (EESM) or mutual information effective SNR mapping (MIESM) method discussed in 2.5.4. In the case of precoding, a single wideband precoder is selected and reported when wideband precoding is requested by the eNodeB.

When better frequency domain scheduling resolution is desired the eNodeB can configure the UE for sub-band reporting, as shown in figure 2.35. Sub-band reporting is used to support LTE's frequency selective scheduling (FSS) feature which was standardized to 
help overcome frequency selective channels. The sub-band or wideband SNRs are mapped to one or more modulation and coding schemes (MCS) that will achieve the LTE required block error rate (BLER) of $10 \%{ }^{6}$ for the allocated band(s). Figure 2.35 shows how the modulation order is varied to match the channel conditions in the sub-bands across the downlink bandwidth.

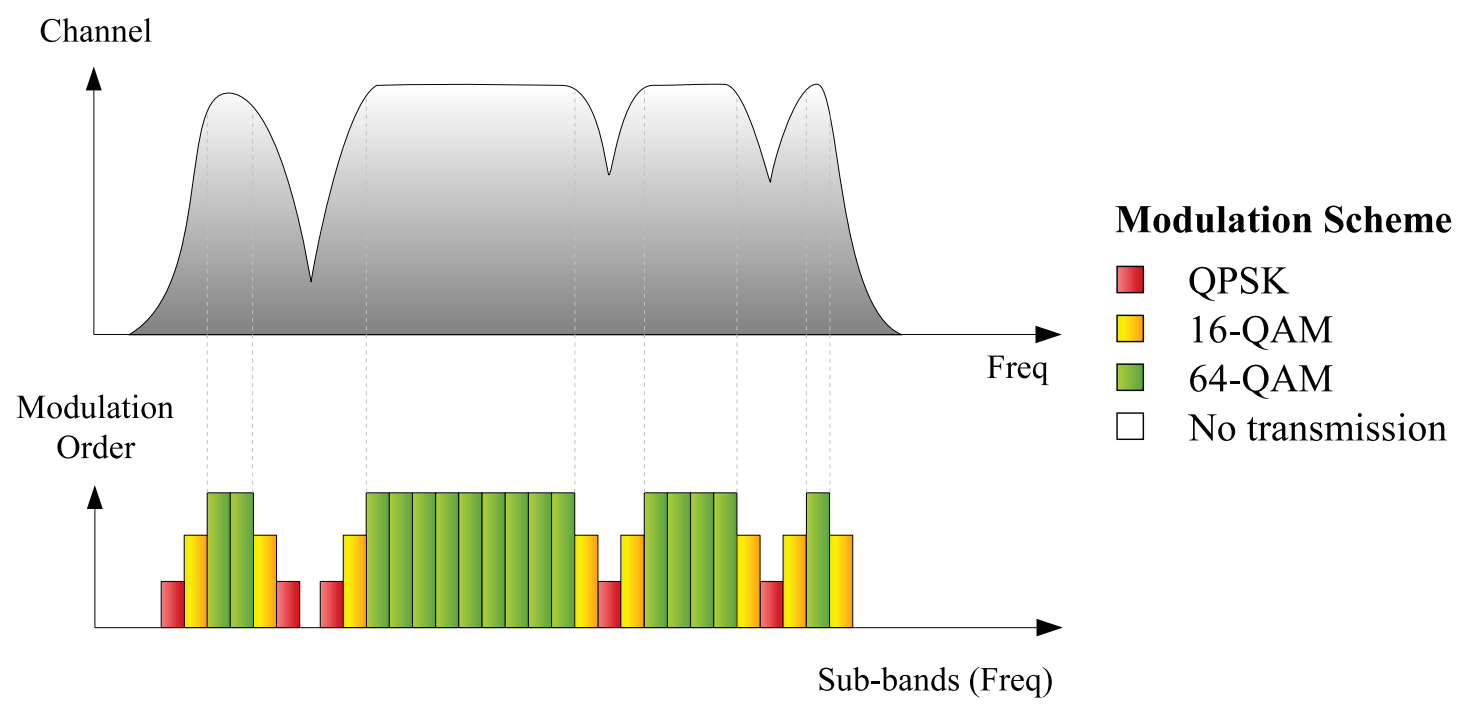

Figure 2.35: Frequency Domain Representation of Sub-bands and Sub-band CQI Reporting

\section{Feedback Reporting}

Feedback reporting is the transmission of the channel state information measured at the UE to the base station to allow the base station to track the changing channel. The UE-preferred MCS, rank and precoder are sent to the base station on one of two uplink channels; the physical uplink control channel (PUCCH) or the physical uplink shared channel (PUSCH). The downlink channel state indicators are reported by the UE on the PUCCH for periodic reporting or the PUSCH when a-periodic reports are requested by the eNodeB.

The UE communicates its preferred modulation, coding, rank and precoding scheme to the base station using quantized indexes which represents the UE's preferred channel state

\footnotetext{
${ }^{6}$ The physical layer block error rate (BLER) limit is given in section 7.2.3 of [22] and is measured after turbo decoding, block reconstruction and CRC checking, but before the HARQ at the MAC layer. The new CQI for downlink transmission is selected by finding the transport block and modulation and coding scheme that can be transmit in the allocated resource blocks while satisfying the 10\% BLER limit imposed at the physical layer.
} 
indicators. The UE maps the chosen MCS to the corresponding CQI, the precoder to the precoding matrix indicator (PMI) and the rank to the rank indicator (RI). The CQI directs the base station to use a predefined set of modulation and coding schemes shown in table A.1. Similarly, the PMI is used to indicate to the base station which precoder to use while accounting for the rank and the transmission mode. The use of the precoder and PMI are detailed in section 6.3 .4 of [1].

Table 2.8 is a summary of the channel state information that may be included in the uplink reports sent to the base station. The measurement type for each channel state indicator is listed in the second column, and the corresponding downlink control information (DCI) sent to the UE via the PDCCH is listed in the fourth column. The particular channel state information that is sent to the UE depends on the transmission mode in use.

Table 2.8: Channel State Information Reported by the UE

\begin{tabular}{cccc}
\hline $\begin{array}{c}\text { Channel State } \\
\text { Information }\end{array}$ & Measurement & Indicator & $\begin{array}{c}\text { Downlink Control } \\
\text { Information Type }\end{array}$ \\
\hline \hline $\begin{array}{c}\text { Channel } \\
\text { Quality }\end{array}$ & $\begin{array}{c}\text { Sub-band SNR or } \\
\text { wideband EESM SNR }\end{array}$ & CQI & $\begin{array}{c}\text { DCI format 1 \& 2 } \\
\text { both A and B }\end{array}$ \\
Rank & $\begin{array}{c}\text { Rank of the channel matrix } \\
H \text { where } r \leq \text { min }\left(N_{t}, N_{r}\right) \\
\text { Capacity maximizing }\end{array}$ & RI & $\begin{array}{c}\text { DCI format 1 \& 2 } \\
\text { both A and B } \\
\text { Precoder }\end{array}$ \\
& $\begin{array}{c}\text { Precoder (diagonalizes the } \\
\text { channel } H=U \Sigma V^{H}\end{array}$ & DCI format 1B \& 2B \\
\hline
\end{tabular}

The knowledge of the CQI and PMI mapping at both the UE and BS means that the complete channel does not need to be reported to the eNodeB. Instead a small number of bits can be used to indicate the desired MCS and precoder to the eNodeB. The CQI and PMI quantization is a necessary step in the transmission of the feedback from the UE to the BS to keep the uplink feedback overhead as low as possible. The quantization of the channel state indicators is especially important to prevent congestion on the uplink when are the cell becomes loaded with many UEs. The feedback reporting can also start to consume a large share of the uplink when the feedback reporting is frequent to support UE mobility and the UEs are using sub-band reporting for multiple codewords. 
The MCS is converted to a 4-bit CQI with a value ranging from 0 to 15 as shown in the 4-bit CQI table, table 7.2.3-1 of [22] and in appendix A.1. A variety of modes can be used to adjust the UE feedback reporting rate based on the UE velocity and the need to track the channel. The reporting modes are listed in tables "7.2.1-1: CQI and PMI Feedback Types for PUSCH Reporting Modes" and "7.2.2-1" of [22] for a-periodic reporting on the $\mathrm{PUSCH}$ and periodic reporting on the PUCCH.

\section{Frequency Selective Scheduling}

In order to overcome a frequency selective channel, LTE includes the opportunistic scheduling feature known as frequency selective scheduling (FSS). The base station has the authority to instruct the UE to report channel state indicators on a sub-band or wideband basis. The benefit of sub-band CQI and PMI reporting is that the eNodeB may use the sub-band channel information to schedule the UEs according to the sub-bands offering the best channel to the UE. An example of a sub-band reporting scheme used in LTE is called " $M$-Best" reporting. As the name implies, in $M$-Best reporting mode the UE reports channel state information for the $M$ best sub-bands. The eNodeB can then use the reported sub-band channel quality indicators to attempt to schedule future downlink transmissions to each UE according to their preferred sub-bands.

\subsection{LTE Transmission Modes}

Transmission modes allow LTE radio access networks to achieve the spectral efficiencies required by operators in order to provide the high bit rates demanded by customers. Each LTE transmission mode has particular strengths and weaknesses and was added to the 3GPP LTE standards after careful consideration. Transmission modes can be selected to make better use of the current channel conditions given sufficient UE and eNodeB capabilities. In LTE, the capabilities of the UE depends on the intended use of the UE, defined by the manufacturer of the product, and the engineering efforts expended by the manufacturers to add the desired feature sets.

The eNodeB establishes the transmission modes used on the PDSCH. The new selection is communicated to the UE on the PDCCH channel and decoded by the UE using its CRNTI. A list of some of the LTE downlink modes are given in table 2.9 and the modes used for downlink performance evaluation are introduced in this section. While transmission mode 1 (TM1) is not evaluated in this study it is briefly discussed for completeness and 
because other modes build on top of TM1 by adding features that help to increase the system efficiency and bit rates.

Table 2.9: LTE Transmission Modes

\begin{tabular}{clcl}
\hline Mode & \multicolumn{1}{c}{ Description } & Layers & \multicolumn{1}{c}{ Antenna Ports } \\
\hline \hline 1 & Single antenna port & 1 & Single antenna (port 0) \\
\hline 2 & Transmit diversity $^{*}$ & 1 & 2 or 4 antennas \\
\hline 3 & Open-loop spatial multiplexing* $^{*}$ & 1 or 2 & $\begin{array}{l}2 \text { antennas (port 0 up to 1) } \\
4 \text { antennas (port 0-3) }\end{array}$ \\
\hline 4 & Closed-loop spatial multiplexing* $^{*}$ & 1 or 2 & $\begin{array}{l}2 \text { antennas (port 0 up to 1) } \\
4 \text { antennas (port 0-3) }\end{array}$ \\
\hline 5 & Multi-user MIMO & 1 or 2 & 2 or 4 antennas \\
\hline 6 & Closed-loop rank-1 beam forming* & 1 & 2 or 4 antennas \\
\hline 7 & Single-layer beamforming & 1 & 8 antennas (port 5) \\
\hline 8 & Dual-layer beamforming & 1 or 2 & $\begin{array}{l}8 \text { antennas (port 7 and 8) } \\
\text { UE-specific pilots }\end{array}$ \\
\hline
\end{tabular}

${ }^{*}$ LTE transmission modes used in this thesis.

\subsubsection{TM1 Single Antenna Transmission}

TM1 is the simplest mode available in LTE. As the transmission mode's name suggests, only a single antenna port is used for transmission at the eNodeB, therefore, only single layer and codeword can be transmit across the channel. This does not limit the number of receiver antennas but it does limit the performance of the cell since MIMO is not possible when using TM1.

\section{Link Adaptation in TM1}

TM1 uses link adaptation to select the optimal MCS to track the changing channel conditions. The cell-specific reference signals are transmit using antenna port 0 across the downlink bandwidth to allow the UE to measure the channel. The UE can then perform 


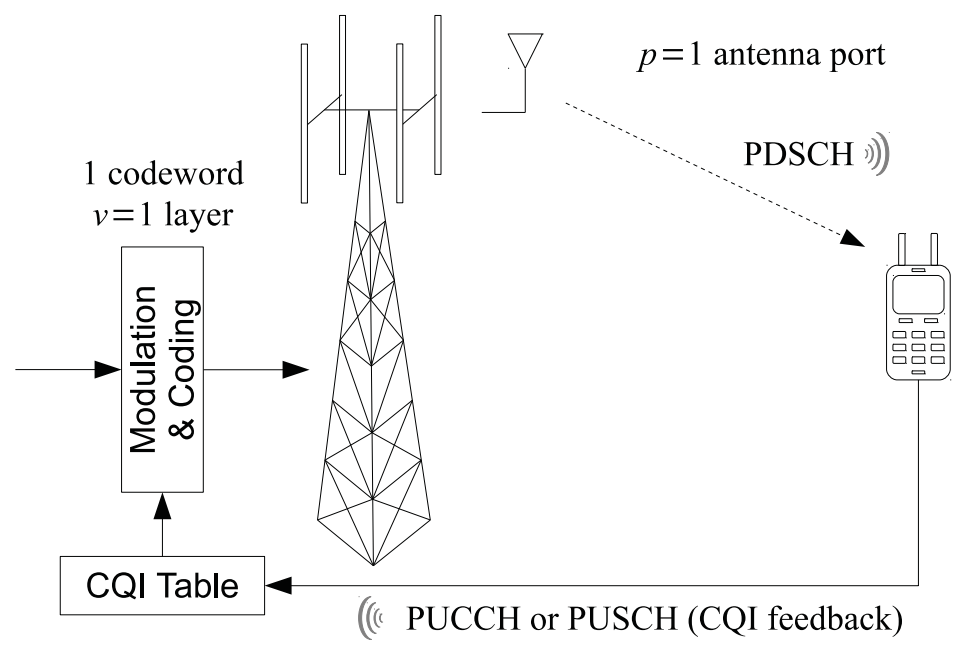

Figure 2.36: Single Antenna Port Transmission

wideband or sub-band CQI reporting for a single layer and codeword in TM1 in order to inform the eNodeB of the preferred modulation and coding scheme.

In TM1 only a single layer is possible and the codeword-to-layer mapping of the complex modulated symbols to layer symbols is direct. The codeword-to-layer mapping is given by 2.55 .

$$
x^{(0)}(i)=d^{(0)}(i)
$$

Due to the lower complexity of TM1's single antenna port transmission scheme, the mode is intended for simple, low cost and low data rate monitoring solutions, like machine to machine (M2M) products.

\subsubsection{TM2 Transmit Diversity}

Transmit diversity is the second transmission mode in LTE and the first mode to make use of multiple antennas. It is used as a primary transmission mode and as a fall-back mode for other transmission schemes due to the robust nature of the scheme used in TM2.

TM2 uses orthogonal codes known as space-frequency block codes (SFBCs) to repeat information symbols on the antenna ports to improve SNR and coverage based on the work of Alamouti in [23]. The orthogonal codes are a form of diversity since two streams of data are transmit and linearly recombined at the receiver. The transmission scheme is known as space-frequency block codes since it makes use of space and frequency diversity when two 
antenna ports are employed. When the eNodeB transmit antennas are sufficiently spaced, the symbols transmit from the different eNodeB antennas experience independent fading and noise. SFBC adds spatial diversity by repeating transformed symbols on multiple antenna ports and the receiver tries to average out the channel effect in a similar fashion as the maximal ratio combining (MRC). The orthogonal codes achieve optimal SNR without the need for channel knowledge at the eNodeB as stated in [24], as well as other advantages listed below.

Advantages of TM2's SFBC transmit diversity scheme are:

- The $2 x 1$ scheme was shown to provide the same diversity as generalized maximalMRC with 1-transmitter and 2-receivers in [23].

- SFBC is useful for channels that do not have feedback reporting.

- Diversity combining improves cell edge performance (coverage) which results in fewer outages and better quality of service (QoS) for customers.

- Improved decoding at the UE due to space-frequency diversity when time diversity is not present in low mobility situations.

- System bandwidth and power does not need to be increased to improve coverage.

- Space-frequency block codes do not add significant decoding delay.

Due to the robust nature of TM2 it is currently used to transmit the control channels, such as the physical broadcast channel (PBCH) and the PDCCH, which must be successfully decoded even under very poor channel conditions. The PBCH and PDCCH use transmit diversity since the UE relies on the successful decoding of the PBCH and PDCCH channels in order to interpret the information in the remaining portion of the sub-frame and other parts of the spectrum. Transmit diversity allows the UE to successfully operate in low SNR areas at the cell edge and combat deep fading due to shadowing or destructive interference occurring in low mobility situations when time diversity is not present.

TM2 is also used for PDSCH transmissions and due to its robust performance, LTE specifies TM2 as a fall-back mode for other transmission modes. TM2 is used as a fallback mode when the current channel conditions do not support the use of higher complexity MIMO transmission modes, like spatial multiplexing. However, a serious limitation of TM2 is that it is not capable of taking advantage of MIMO channel conditions which permit throughput to be increased by sending multiple codewords when multiple information streams are present in the channel. 


\section{Two Antenna Transmit Diversity}

SFBC is a space-frequency version of Alamouti's space-time coding scheme, used to obtain space and frequency diversity. TM2 uses SFBC with two antennas and two sub-carriers to repeat the transmission of complex modulated symbols in space and in the frequency domain as shown in figure 2.37 .

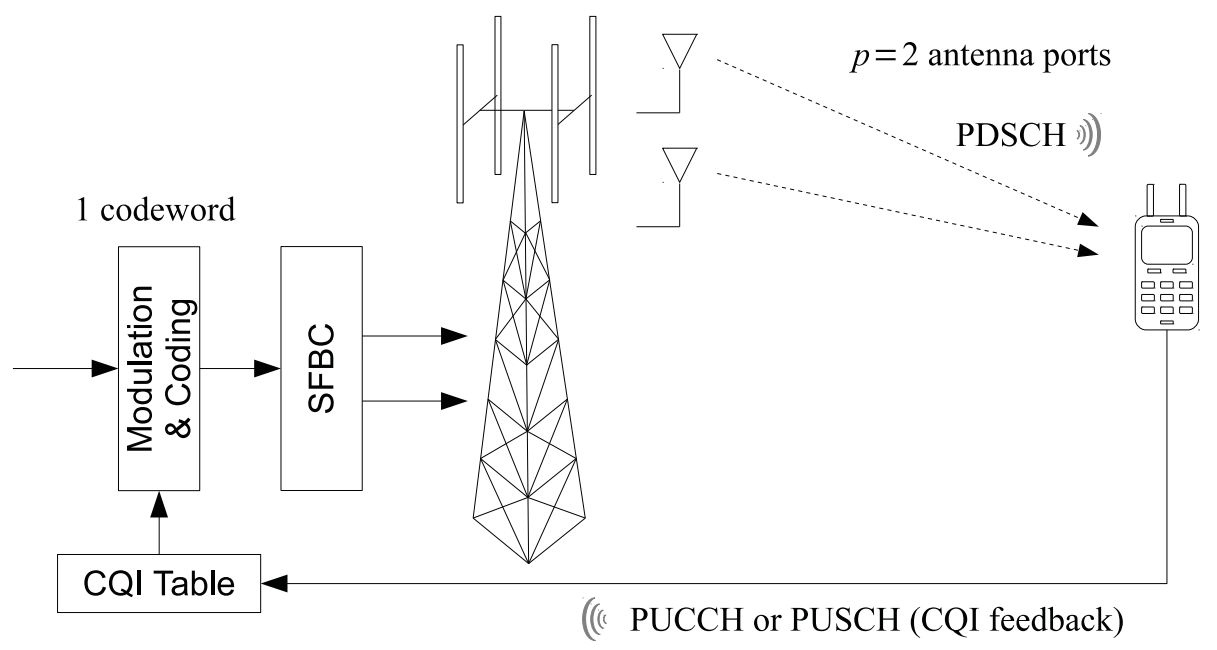

Figure 2.37: Transmit Diversity SFBC Transmissions on 2 Antennas Ports

When using two antenna ports for transmission at the eNodeB the codeword-to-layer mapping is given by table 2.10, an excerpt of table 6.3.3.3-1 of [1]. In table 2.10, the complex symbols $d^{(0)}(2 i)$ and $d^{(0)}(2 i+1)$ of the only codeword are mapped to the two available layer symbols $x^{(0)}(i)$ and $x^{(1)}(i)$. The complex layer symbol are denoted by $x^{(v)}(i)$ where the $i^{\text {th }}$ symbol of layer $v$.

Table 2.10: Codeword-to-Layer Mapping for Transmit Diversity (2 Antenna Ports)

\begin{tabular}{ccc}
\hline Layers & Codewords & Codeword-to-layer Mapping \\
\hline \hline 2 & 1 & $x^{(0)}(i)=d^{(0)}(2 i)$ \\
& $x^{(1)}(i)=d^{(0)}(2 i+1)$ \\
\hline
\end{tabular}

The SFBC scheme shown in 2.56 maps the layer symbols $x^{(1)}(i)$ and $x^{(0)}(i)$ to subcarriers transmit on the antenna ports. In $2.56, y^{(p)}(i)$ is the output of the precoding stage 
for antenna port $p$, where $p \in\{0,1\}$. When referencing the precoded symbols $y^{(p)}(i)$, index $i$ refers to the sub-carrier in the frequency domain, however, $i$ is also used to index the $i^{\text {th }}$ symbol of the each layer. The range for $i$ is $i=0,1 \ldots, M_{\text {symb }}^{\text {layer }}-1$ where $M_{\text {symb }}^{\text {layer }}$ is the number of modulated symbols $d^{(0)}$ for the only codeword.

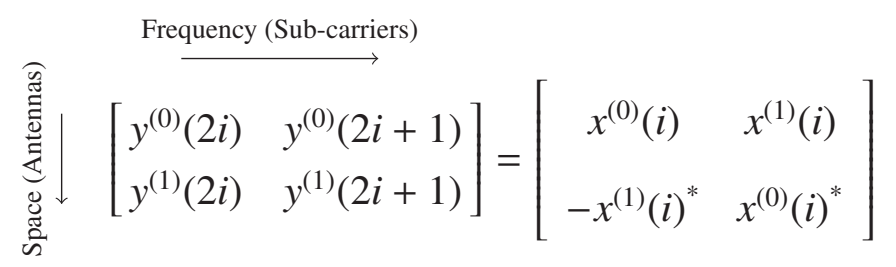

In definition 2.56, SFBC maps the consecutive symbols in time, $x^{(0)}(i)$ and $x^{(1)}(i)$, to the adjacent sub-carriers $y^{(0)}(2 i)$ and $y^{(0)}(2 i+1)$ in the frequency domain of the first antenna port, antenna port 0 . To achieve the optimal SNR, the number of sub-carriers (i.e. symbols to be mapped) is limited by the coherence bandwidth of the channel since the channel must be the relatively constant to perform the necessary algebra to apply the matched filter to the received signals to obtain the transmit symbols. On the second antenna port, antenna port 1 , spatial diversity is obtained by transmitting $-x^{(1)}(i)^{*}$ and $x^{(0)}(i)^{*}$.

\section{Four Antenna Transmit Diversity}

In the case of four transmit antennas at the eNodeB, SFBC is combined with frequencyswitched transmit diversity (FSTD). The SFBC-FSTD scheme was standardized after the evaluation of other transmit diversity coding schemes. The pure SFBC scheme could not be used since it would not lead to an orthogonal code, therefore the SFBC-FSTD scheme was chosen.

The codeword-to-layer mapping for the four antenna port base station configuration is given by table 2.11, an excerpt of table 6.3.3.3-1 of [1]. In table 2.11, the complex symbols $d^{(0)}(2 i), d^{(0)}(2 i+1), d^{(0)}(2 i+2)$ and $d^{(0)}(2 i+3)$ of the only codeword are mapped to the four available layer symbols $x^{(0)}(i), x^{(1)}(i), x^{(2)}(i)$ and $x^{(3)}(i)$ as shown. The complex layer symbol are denoted by $x^{(v)}(i)$ where the $i^{\text {th }}$ symbol of layer $v$.

The SFBC-FSTD scheme applies SFBC to two pairs of symbols, $\left\{x^{(0)}(i), x^{(1)}(i)\right\}$ and $\left\{x^{(2)}(i), x^{(3)}(i)\right\}$, across four antenna ports. The pairs of symbols are alternated when applied to the four antenna ports and the pattern is repeated across the frequency domain. In order to avoid interference from other symbols the pairs of SFBC symbols are transmit on a pair of sub-carriers on antenna port pairs $\{0,2\}$ or $\{1,3\}$. The other antenna port pair is silent on 


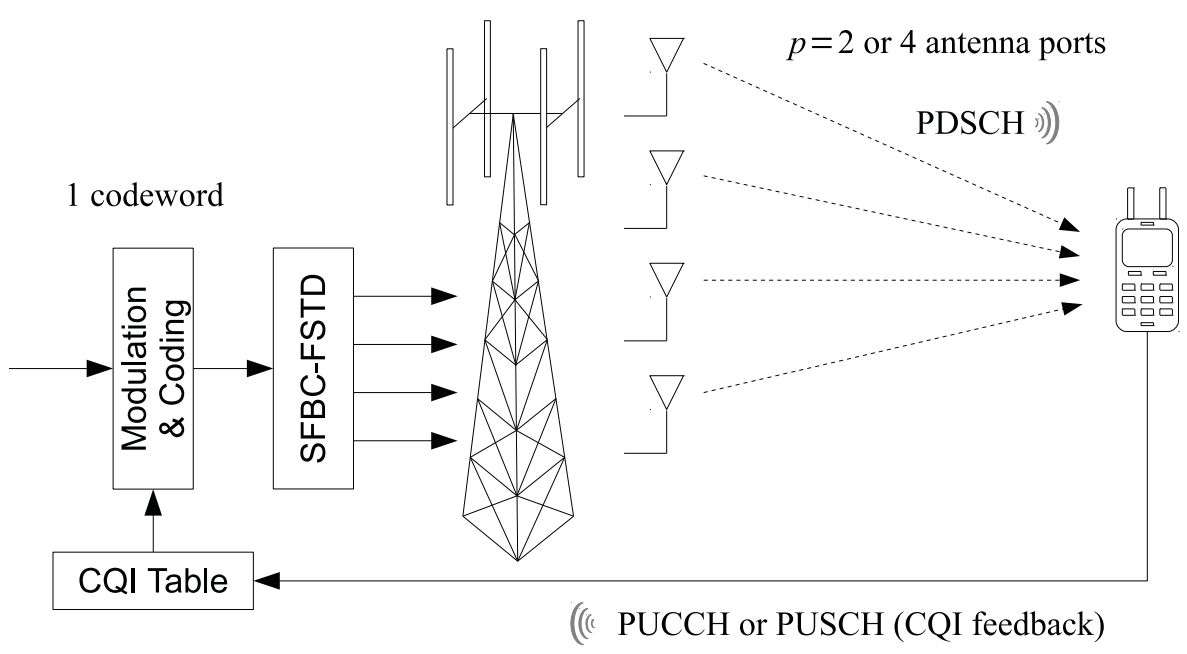

Figure 2.38: Transmit Diversity SFBC-FSTD Transmissions using 4 Antenna Ports

Table 2.11: Codeword-to-Layer Mapping for Transmit Diversity (4 Antenna Ports)

\begin{tabular}{|c|c|c|}
\hline Layers & Codewords & Codeword-to-layer Mapping \\
\hline \multirow{4}{*}{4} & \multirow{4}{*}{1} & $x^{(0)}(i)=d^{(0)}(4 i+0)$ \\
\hline & & $x^{(1)}(i)=d^{(0)}(4 i+1)$ \\
\hline & & $x^{(2)}(i)=d^{(0)}(4 i+2)$ \\
\hline & & $x^{(3)}(i)=d^{(0)}(4 i+3)$ \\
\hline
\end{tabular}


those same frequency resources in order to keep the transmissions from interfering with each other as shown in 2.57. In 2.57, the $y^{(p)}(k)$ is the symbol transmit at sub-carrier $k$ on antenna port $p, i^{\prime}=4 i$ is the index of the first of four sub-carriers defined by the pattern and $i$ is the index of the complex layer symbol.

$$
\begin{aligned}
& \text { Frequency (Sub-carriers) }
\end{aligned}
$$

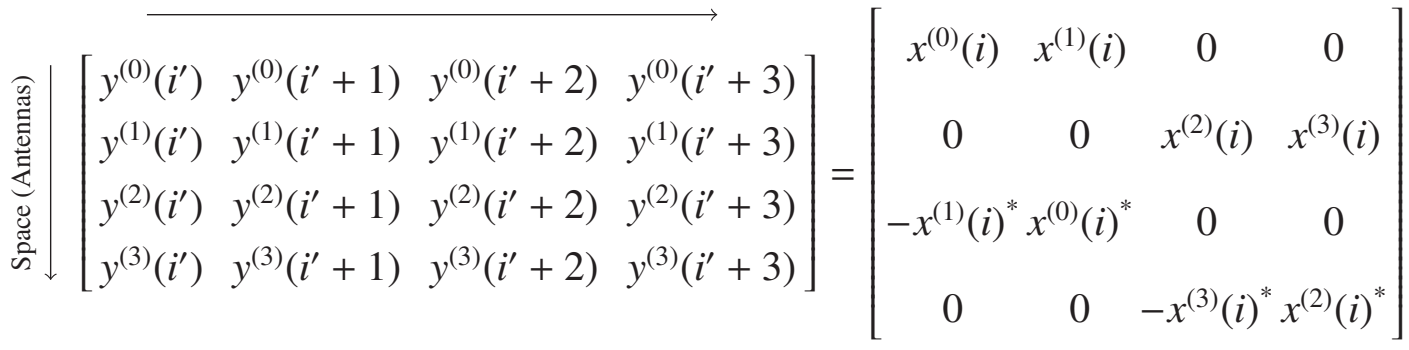

When using four antenna ports in LTE, the reference signal pattern of antenna ports $\{2,3\}$ is less dense than the reference signal pattern defined for antenna ports $\{0,1\}$, as seen in figure 2.31 . The complete channel estimate for ports $\{2,3\}$ could potentially suffer inaccuracies, under certain channel conditions, due to the sparse reference signal pattern used for channel estimation. A benefit of the SFBC-FSTD scheme is that it transmits pairs of symbols on an antenna port with a dense RS pattern and a sparse RS pattern simultaneously. For example, symbols $\left\{x^{(0)}(i), x^{(1)}(i)\right\}$ are transmit on antenna ports $\{0,2\}$, a port with a dense RS pattern and a port with a sparse RS pattern, as discussed in [25], to balance the layer symbol pairs $\left\{x^{(0)}(i), x^{(1)}(i)\right\}$ and $\left\{x^{(2)}(i), x^{(3)}(i)\right\}$ across accurate and potentially inaccurate channel estimates.

\section{Link Adaptation in TM2}

TM2 performs channel estimation and reports the requested channel state information to the eNodeB on the uplink channels. When the eNodeB is specifically set to use TM2, the uplink feedback can be kept low since only the CQI is used to adapt the PDSCH to the current channel conditions. TM2 benefits from not needing to rely on the RI and the PMI since these indicators can become unreliable as the UE velocity increases.

The increased reporting feedback required for spatial multiplexing and beamforming modes discussed in sections 2.5.3, 2.5.4 and 2.5.5 (TM3, TM4, TM6) is an advantage over TM2 at low velocity but becomes a burden and disadvantage at high reporting rate and high velocities and is the reason why TM2 is still a relevant primary mode for transmission and 
also as the fall-back mode for other LTE transmission modes.

\subsubsection{TM3 Open-Loop Spatial Multiplexing}

TM3 is a spatial multiplexing mode defined in the 3GPP LTE standards which provides the basic link adaptation features (adaptive modulation and coding (AMC)) seen in previous transmission modes and also includes spatial multiplexing to achieve the high bit rates desired by consumers and operators.

TM3 is the first of the spatial multiplexing modes and the first mode which is capable of transmitting multiple codewords, as shown in figure 2.39, when the reported channel rank is $\geq 2$. Since the rank is limited by the number of antennas at both the UE and the eNodeB, a key requirement of the spatial multiplexing transmission modes, including TM3, is the availability of multiple antennas at both the UE and the eNodeB. Spatial multiplexing improves the radio access network efficiency by transmitting concurrent streams of data from the eNodeB when two or more independent data streams are present in the channel.

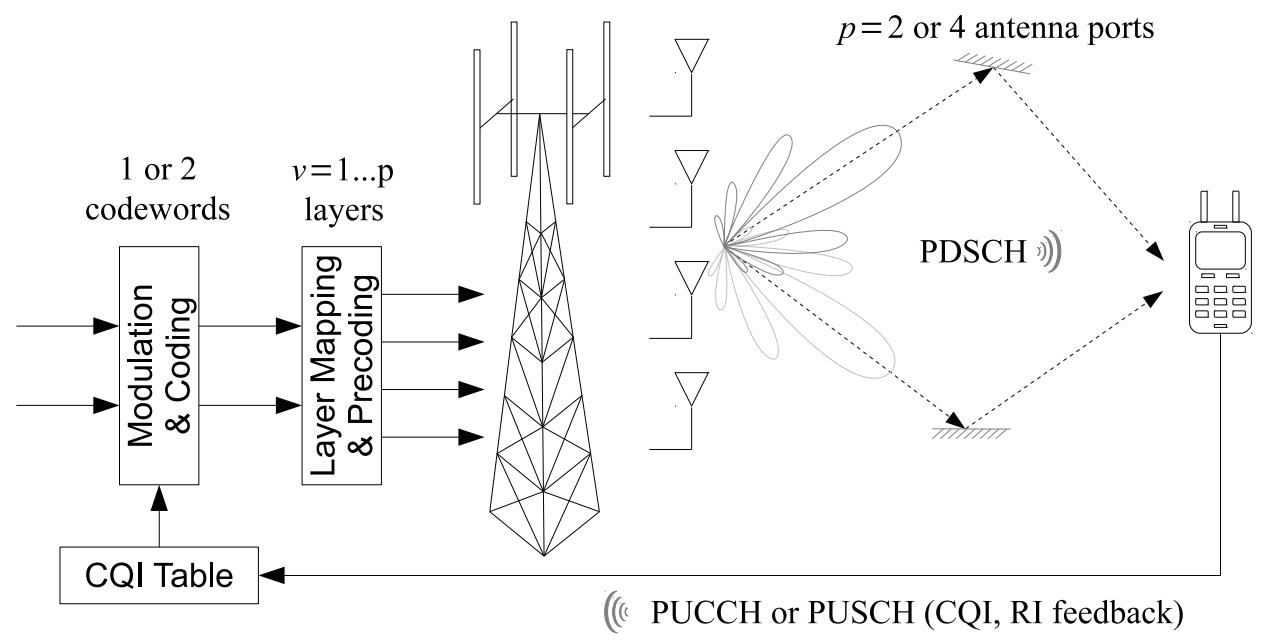

Figure 2.39: Open-Loop Spatial Multiplexing (OLSM)

The MIMO characteristics of the channel are determined by the UE using channel estimation and analysis after the reception of previous downlink transmissions. The UE reports the CQI and the rank indicator (RI) to the eNodeB where the eNodeB decides the parameters of the next spatial multiplexing transmission.

The UE reported rank indicator (RI) is used at the eNodeB to decide the number of codewords to use to send information in the available time-frequency resources. TM3 also 
performs beamforming but the UE does not report the optimal precoding matrix in the form of the PMI to the eNodeB, such as in TM4. The lack of PMI reporting to the eNodeB means that information about the optimal precoder selection is not available at the eNodeB, therefore, TM3 must use a fixed or pseudo random cycling precoder for transmission. TM3 is therefore called open-loop spatial multiplexing (OLSM) due to the open-loop operation of the precoder and the resulting beam patterns generated by the eNodeB transmissions. The open-loop precoding found in TM3 is the primary difference between TM3 and TM4 (discussed in section 2.5.4). The throughput performance of TM3 and TM4 depends on a number of factors, however, TM4 can outperform TM3 when a channel matching precoder is used at the eNodeB for transmission. On the other hand, TM3 can be more effective than TM4 if the precoder is not well matched to the channel due to PMI aging, infrequent reporting or other impairment affecting link adaptation performance.

\section{Downlink Signaling for TM3}

In TM3, the 3GPP LTE standard permits the eNodeB to switch between the robust transmit diversity transmission scheme or offer higher data rates by making use of the radio access network's spatial multiplexing transmission capabilities. To inform the UE of the transmission scheme, downlink control information (DCI) is transmitted by the eNodeB to the UE in the control channel portion of the downlink transmissions. The DCI format indicates to the UE whether TM3's open-loop spatial multiplexing transmission scheme or transmit diversity was used for the PDSCH transmission. Table 2.12 is the portion of table 7.1-5 from [22] which pertains to TM3. Table 2.12 shows the DCI formats used by the eNodeB in TM3 to indicate to the UE how to decode the PDSCH.

Table 2.12: TM3 PDSCH Transmission Scheme

\begin{tabular}{cccc}
\hline Mode & DCI Format & Search Space & Transmission Scheme \\
\hline \hline \multirow{2}{*}{ TM3 } & DCI 1A & CRS \& UE specific by C-RNTI & Transmit diversity \\
\cline { 2 - 4 } & DCI 2A & UE specific by C-RNTI & OLSM or Transmit diversity \\
\hline
\end{tabular}

TM3 uses spatial multiplexing with large delay cyclic delay diversity (CDD) when the UE reports favourable channel conditions (medium to high SNR with a reported rank greater than one) as discussed in section 2.5.3. The robust orthogonal codes used in SFBC 
or SFBC-FSTD become redundant in medium to high SNR scenarios where spatial multiplexing with CDD can be used to transmit two codewords to make better use of the channel resources and increase the throughput.

\section{Link Adaptation in TM3}

In TM3 the UE reports the rank using the rank indicator (RI) and the channel quality using the CQI. Both the RI and the CQI are determined by analyzing the channel estimate obtained from the reference signals (CRS) that are broadcast across the system bandwidth by the eNodeB from each antenna port. The CQI and RI channel state indicators are reported via the PUCCH in "periodic" feedback mode ${ }^{7}$ or on the PUSCH when the UE is configured for frequent reporting in "a-periodic" reporting mode ${ }^{8}$.

The channel rank is used by the eNodeB to decide the number of codewords and layers that will be used on the next downlink transmission. If the UE-preferred rank is greater than one, then one CQI is reported per codeword for wideband reporting and multiple CQI are reported per codeword when sub-band reporting is used.

Sub-band reporting at the UE is configured by the eNodeB when more information about the channel can be beneficial for scheduling downlink transmissions to the UEs located in the cell. When sub-band reporting is used, the downlink bandwidth is partitioned according to table 2.13, taken from [22], where the sub-band size $k$ is defined as a function of the total number of physical resource blocks (PRBs) in the downlink bandwidth $N_{R B}^{D L}$.

\footnotetext{
${ }^{7}$ Mode 1-0 is used for wideband CQI periodic reporting and Mode 2-0 is used for UE selected sub-band CQI periodic reporting in TM3.

${ }^{8}$ Mode 2-0 is used for UE selected sub-band CQI a-periodic reporting and Mode 3-0 is used for higher layer-configured sub-band CQI sub-band a-periodic reporting in TM3.
} 
Table 2.13: Sub-Band Size vs. System Bandwidth

\begin{tabular}{cc}
\hline $\begin{array}{c}\text { System Bandwidth } \\
N_{R B}^{D L}\end{array}$ (PRBs) & $\begin{array}{c}\text { Sub-band Size } \\
\text { (PRBs) }\end{array}$ \\
\hline \hline $6-7$ & - \\
$8-10$ & 4 \\
$11-26$ & 4 \\
$27-63$ & 6 \\
$64-110$ & 8 \\
\hline
\end{tabular}

The main purpose of sub-band CQI reporting is to provide the eNodeB with enough channel quality information spread across the frequency domain to make use of LTE's frequency selective scheduling (FSS) feature. FSS is known as an opportunistic scheme since it tries to maximize the use of the channel by allocating the best resource blocks available to each UE and avoid scheduling UEs in resource blocks that are affected by frequency selective fading. By providing the eNodeB with sub-band CQI values for each sub-band or the "M-Best" sub-bands in the frequency domain, the eNodeB is able to schedule the UEs in the sub-bands that offer the best channel quality, leading to more efficient use of the spectral resources.

\section{Codeword-to-Layer Mapping}

The maximum number of downlink spatial multiplexing layers in LTE depends on the LTE release and the UE category, determined by the parameter UE-category. The codewordto-layer mapping is limited to one layer for a category $1 \mathrm{UE}$, two layers when then $U E$ category parameter is in the range of 2 to 4 and four layers for a category 5 UE. The UE-category parameter determines the UE capabilities and sets the maximum number of layers on a device basis in order to distinguish between low cost or low complexity UEs and UEs that support advanced LTE features.

The codeword-to-layer mapping in LTE was chosen as a trade off between overhead and performance. Layers are limited by the channel rank and number of antenna ports but could be as high as four layers for a category $5 \mathrm{UE}$, as shown in table 2.14.

The maximum number of codewords is limited to two despite the possibility of a higher number of codewords when using four or more antenna elements in the case of the category 
five UE. This is to ensure that link adaptation CSI feedback does not overwhelm the uplink channels since each codeword is required to report multiple channel state indicators for each sub-band, when in sub-band reporting mode.

Table 2.14: Downlink Physical Layer Parameters Set By Parameter UE-category

\begin{tabular}{ccc}
\hline UE Category & LTE Release & $\begin{array}{c}\text { Maximum Number of } \\
\text { Spatial Multiplexing Layers }\end{array}$ \\
\hline \hline 1 & 8 & 1 \\
$2,3,4$ & 8 & 2 \\
5 & 8 & 4 \\
6,7 & 10 & 2 or 4 \\
8 & 10 & 8 \\
\hline
\end{tabular}

Table 2.15 shows the codeword-to-layer mapping for spatial multiplexing transmissions. The codewords are transmit on 1 or 2 layers. Subsequently, the layers are mapped to antenna ports as described in the

Table 2.15: Downlink Physical Layer Codeword-to-Layer Mapping

\begin{tabular}{ccc}
\hline Reported Channel Rank & Codeword 1 & Codeword 2 \\
\hline \hline Rank 1 & Layer 1 & NA \\
Rank 2 & Layer 1 & Layer 2 \\
Rank 3 & Layer 1 & Layer 2 and 3 \\
Rank 4 & Layer 1 and 2 & Layer 2 and 3 \\
\hline
\end{tabular}

\section{Precoding in TM3}

When using open-loop spatial multiplexing (OLSM), the UE-preferred precoding matrix is not reported by the UE, hence, the open-loop nature of TM3. Despite the lack of PMI feedback in OLSM, precoding is still used but the eNodeB performs the precoding task without knowledge of the UE's preferred precoder. To ensure the eNodeB and the UE 
apply the appropriate transmit and receive beamforming matrices for open-loop spatial multiplexing, 3GPP defined a fixed and cyclic precoding scheme for OLSM in TM3 which is known at both the eNodeB and the UE.

Precoding is the operation of mapping the complex layer symbols to the antenna ports. The layer symbols can be directly mapped to individual antennas or combinations of symbols can be spread out across the antenna array. The latter is beneficial because beamforming can be performed by mapping the symbols to multiple antennas and then shaping the radiated energy by delaying the signals of other antennas with reference to the first antenna.

Precoding is achieved by the use of equation 2.58. In equation $2.58, W(i)$ is a $P \times v$ matrix which applies the desired precoding matrix, also known as the transmit beamforming scheme. The precoding matrix $\boldsymbol{W}(i)$ is selected from table 2.16 when two antenna ports are used or table 2.17 when four antenna ports are used.

$$
y=W(i) x
$$

In the expanded precoding equation shown in equation 2.59, the layer symbols, $x^{(v)}(i)$, are transformed to the precoded symbols, $y^{(p)}(i)$, using the selected precoding matrix. The precoding step defined in equation 2.59 is for the case where open-loop spatial multiplexing does not use CDD. Precoding with CDD will be discussed in section 2.5.3.

$$
\left[\begin{array}{c}
y^{(0)}(i) \\
y^{(1)}(i) \\
\vdots \\
y^{(P-1)}(i)
\end{array}\right]=\boldsymbol{W}(i)\left[\begin{array}{c}
x^{(0)}(i) \\
x^{(1)}(i) \\
\vdots \\
x^{(v-1)}(i)
\end{array}\right]
$$

The precoding step uses equation 2.59 to modify the phase of the transmit signal at each antenna port. Due to constructive and destructive interference of the radiated energy from each antenna element the radiation pattern of the antenna array is modified by the precoding matrix.

When beamforming is leveraged by the eNodeB the cell coverage can be extended by selecting a precoder and resulting beam pattern that focuses the radiated energy from the base station antenna array in the direction of the UE, as shown in figure 2.41 for two antenna ports. This is particularly beneficial under low UE velocity conditions when using closedloop spatial multiplexing (described in section 2.5.4) because the UE reports the optimal 
precoding matrix indicator to the eNodeB. Despite the lack of the optimal PMI reports in TM3, the 3GPP included open-loop spatial multiplexing in the LTE standard because conditions do exists where OLSM can perform better than CLSM, such as when the PMI is unreliable due to a rapidly changing channel.

Another benefit of beamforming in radio access networks is that it can increase the directivity of the radiated field. The increase in the directivity reduces interference to other spatially separated UEs and permits greater frequency reuse in a given area. In addition, the spatially independent streams can be used to transmit multiple codewords to one or more UEs thus improving the system efficiency.

\section{Large-Delay Cyclic Delay Diversity (LD-CDD)}

In LTE, cyclic delay diversity (CDD) is applied to the transmitted OFDM symbols when the rank used for downlink transmission is greater than one. LTE uses CDD with openloop spatial multiplexing to artificially increase the frequency selectivity of a potentially frequency flat channel. In a slowly changing frequency flat channel with poor channel conditions, a large number of continuous sub-carriers can be affected by fading or shadowing across a number of OFDM symbols in time. If a significant portion of a sub-frame is affected, one or more transport blocks may fail to decode successfully due to the large number of bit errors concentrated in parts of a transport block or codeword. CDD helps to randomize the effect of the frequency flat channel by applying delays to different subcarriers across the frequency domain. The delays help to increase the frequency selectivity of the frequency flat channel which helps to randomize and spread the bit errors when interleaving is used and makes it easier for the decoder to decode the transmit codeword, as suggested in [26].

When CDD is used, the eNodeB transmits delayed versions of the same OFDM subcarriers from each antenna port. The cyclic nature of the transmitted OFDM symbol is maintained since the phase delay is applied before the $\mathrm{CP}$ is added as shown in 2.40.

The implementation of CDD is given by equation 2.60. $W(i)$ is a $P \times v$ matrix which applies the desired transmit beamforming scheme and is selected from table 2.16 when two antenna ports are used or table 2.17 when four antenna ports are used. In the case of 2 antennas, the beamforming precoder is fixed to index 0 , corresponding to the first row of table 2.16, and the matrix is selected by the number of layers $v$ used for transmission. 


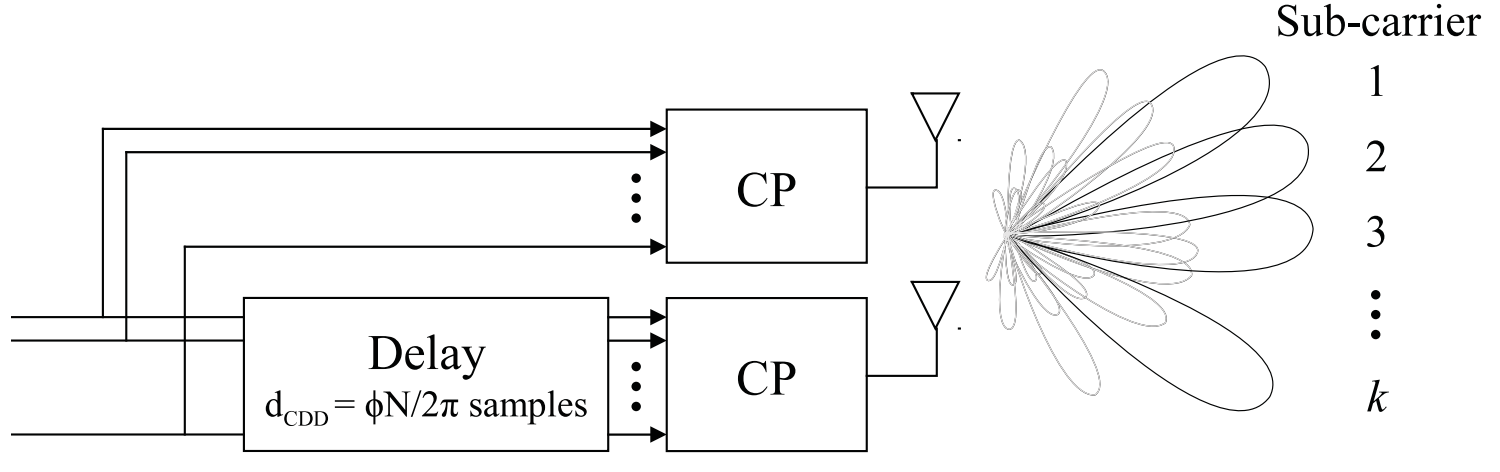

Figure 2.40: Addition of CDD to Transmitter Chain and Resulting Sub-Carrier Level Beamforming

The matrix $\boldsymbol{D}(i)$ and $\boldsymbol{U}$ apply the CDD scheme to the OFDM symbol before the CP is prepended to the OFDM symbol. The CDD matrix $\boldsymbol{D}(i)$ is a diagonal matrix of size $v \times v$ with delays along the diagonal selected to delay the signal from each antenna port independently.

$$
y=W(i) D(i) U x
$$

The symbols transmit on each antenna port, $y^{(p)}(i)$, are beamformed and delayed versions of the symbols after layer mapping, $x^{(v)}(i)$, as shown in equation 2.61.

$$
\left[\begin{array}{c}
y^{(0)}(i) \\
y^{(1)}(i) \\
\vdots \\
y^{(P-1)}(i)
\end{array}\right]=\boldsymbol{W}(\boldsymbol{i})\left[\begin{array}{cccc}
1 & 0 & \ldots & 0 \\
0 & e^{j \phi_{1} k} & \ldots & 0 \\
\vdots & \vdots & \ddots & \ldots \\
0 & 0 & 0 & e^{j \phi_{v-1} k}
\end{array}\right] \boldsymbol{U}\left[\begin{array}{c}
x^{(0)}(i) \\
x^{(1)}(i) \\
\vdots \\
x^{(v-1)}(i)
\end{array}\right]
$$

The time delay, applied by $\boldsymbol{D}(\boldsymbol{i})$, results in a linearly increasing phase shift in the frequency domain as the frequency increases across the transmission bandwidth. The subcarriers add constructively and destructively across the system bandwidth increasing the frequency selectivity of the channel and randomizing the error distribution seen by a decoder. The increase in frequency selectivity caused by CDD helps to overcome flat fading channels where errors can be sequential and more difficult for some decoding algorithms to correct. For instance, in LTE, CDD can improve forward error correction (FEC) performance by ensuring that adjacent sub-carriers are affected differently by the channel effectively randomizing the errors in the received codeword. The artificial frequency selectivity 
added by CDD permits coding gains to be achieved because the FEC scheme used in LTE, composed of the turbo encoder/decoder and the quadratic permutation polynomial (QPP) interleaver, performs better when presented with random errors than when presented with a series of sequential errors, known as errors bursts.

\section{Precoding using Two Antenna Ports at the Base Station}

When two antenna ports are used at the eNodeB in TM3, precoding is performed without applying cyclic delay diversity (CDD). The base station uses equation 2.58 to transform the complex layer symbols to the symbols transmit on the antenna ports before IFFT is performed. The precoding matrix $\boldsymbol{W}(\boldsymbol{i})$ is selected from table 2.16. For two antenna ports, the 3 GPP fixed the precoding matrix to codebook index 0 and the matrix is selected based on the number of layers $v$ used for transmission.

Table 2.16: Precoder Codebook for Transmission on Two Antenna Ports

\begin{tabular}{ccc}
\hline $\begin{array}{c}\text { Codebook } \\
\text { Index }\end{array}$ & \multicolumn{2}{c}{ Number of Layers } \\
\cline { 2 - 3 } 0 & $\frac{1}{\sqrt{2}}\left[\begin{array}{l}1 \\
1\end{array}\right]$ & $\frac{1}{\sqrt{2}}\left[\begin{array}{cc}1 & 0 \\
0 & 1\end{array}\right]$ \\
1 & $\frac{1}{\sqrt{2}}\left[\begin{array}{c}1 \\
-1\end{array}\right]$ & $\frac{1}{2}\left[\begin{array}{cc}1 & 1 \\
1 & -1\end{array}\right]$ \\
2 & $\frac{1}{\sqrt{2}}\left[\begin{array}{c}1 \\
j\end{array}\right]$ & $\frac{1}{2}\left[\begin{array}{cc}1 & 1 \\
j & -j\end{array}\right]$ \\
3 & $\frac{1}{\sqrt{2}}\left[\begin{array}{c}1 \\
-j\end{array}\right]$
\end{tabular}

When two antenna ports are used at the eNodeB, four beamforming patterns are defined for single layer transmission, while two beamforming patterns and a direct layer-to-antenna port mapping are available for two layer transmissions as shown in figure 2.41.

For single layer transmissions the codeword is distributed evenly across the two antenna ports before being transmit as described by the matrix at codebook index 0 of table 2.16. The resulting beam pattern is shown at index 0 of figure $2.41 \mathrm{a}$. On the other hand, when 


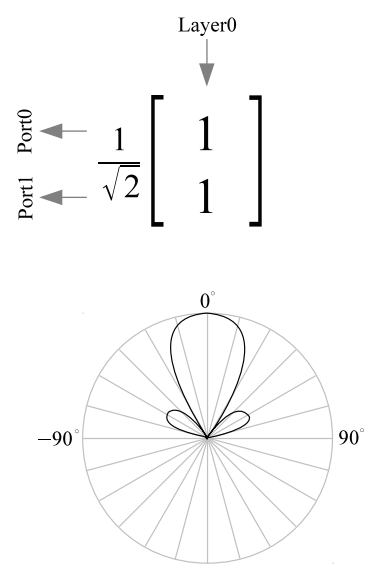

Index 0
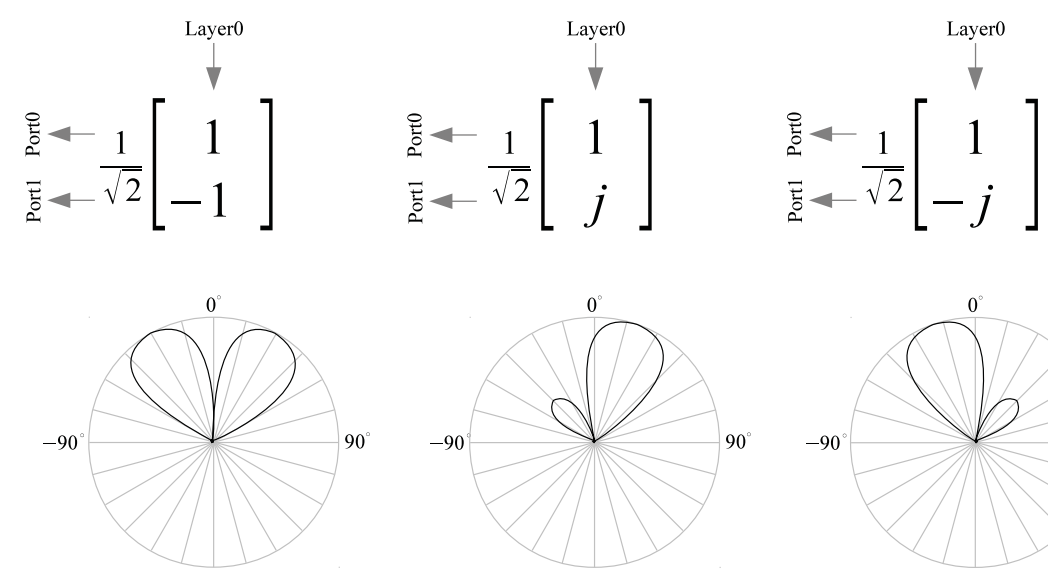

Index 1

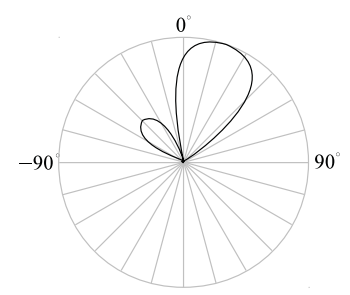

Index 2

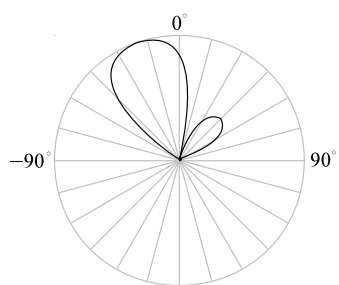

Index 3

(a) Beamforming Patterns using a Single Transmission Layer

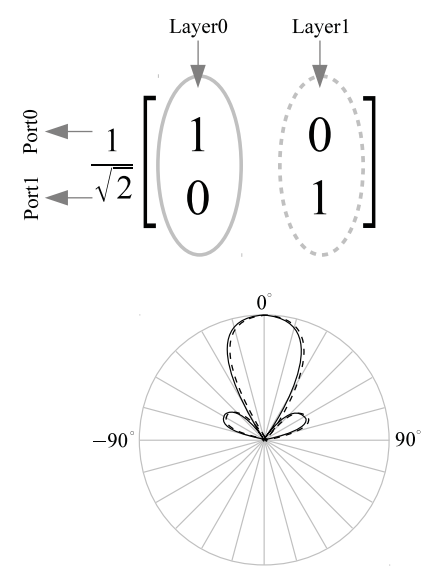

Index 0 (not used for BF)
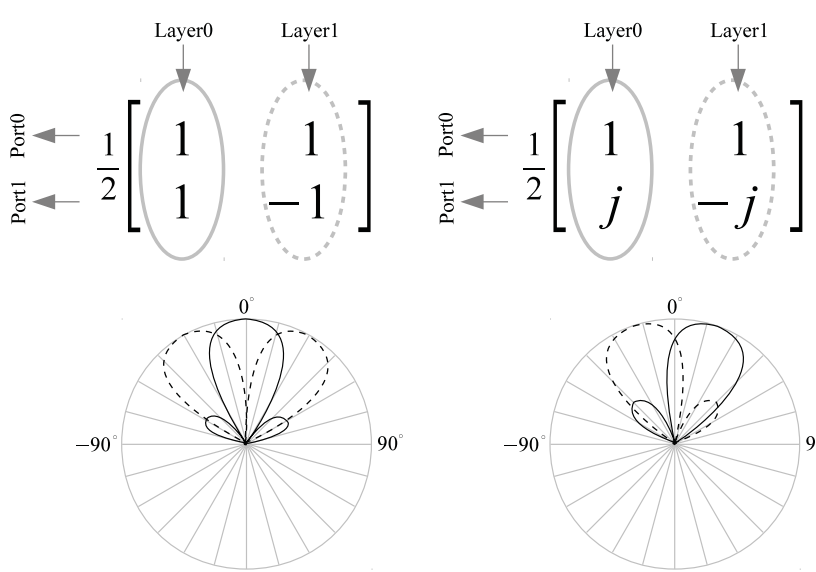

Index 1

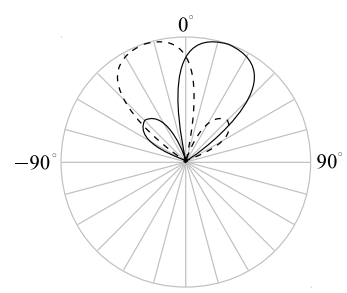

Index 2

(b) Beamforming Patterns using Two Transmission Layers

Figure 2.41: Beamforming Patterns for a Two Antenna Base Station Configuration 
two transmission layers are used for downlink transmissions the fixed precoding matrix for two layer transmissions at codebook index 0 in table 2.16 maps the individual layers to separate antenna ports. In this case, no beamforming is achieved and the scheme assumes the two antenna ports are capable of producing two independent data streams based on the rank reported by the UE.

\section{Precoding using Four Antenna Ports at the Base Station}

When the eNodeB antenna array is composed of four antenna elements it is possible to use four antenna ports for downlink transmissions. Four antenna ports permit the eNodeB to transmit up to four layers. The benefit of using four antenna ports over the two antenna port scheme, described in section 2.5.3, is an increase in the number of possible beam patterns that can be used and an increase in the directivity of some beam patterns.

The codebook shown in table 2.17 was defined by the 3GPP in table 6.3.4.2.3-2 of [1] for spatial multiplexing transmissions using four antenna ports at the eNodeB. The 16 precoding matrices shown in table 2.17 allow the UE and eNodeB to select a precoder from a larger set of beam patterns. In addition, four antenna ports offers a wider variety of two stream beam pattern combinations. With the added directivity and resolution of four antenna port transmit beamforming the expectation is that spatial multiplexing using four antenna port should perform better than spatial multiplexing using two antenna ports.

\section{Advantages of Open-Loop Spatial Multiplexing}

The lack of PMI feedback means open-loop spatial multiplexing has lower signaling overhead and is of lower complexity than closed-loop spatial multiplexing, but implies that open-loop spatial multiplexing may not be able to achieve the same performance of closedloop spatial multiplexing in certain channel conditions ${ }^{9}$. While open-loop spatial multiplexing may not adapt the precoder to the channel, the following benefits were considered by the 3GPP when the decision was taken to include OLSM as the third transmission mode.

\footnotetext{
${ }^{9}$ Closed-loop spatial multiplexing can achieve better performance than open-loop spatial multiplexing when the PMI reporting is capable of tracking the channel adequately, such as when the UE velocity is low.
} 
Table 2.17: Precoder Codebook for Transmission on Four Antenna Ports From [1]

\begin{tabular}{|c|c|c|c|c|c|}
\hline \multirow{2}{*}{$\begin{array}{l}\text { Codebook } \\
\text { index }\end{array}$} & \multirow{2}{*}{$u_{n}$} & \multicolumn{4}{|c|}{ Number of layers $v$} \\
\hline & & 1 & 2 & 3 & 4 \\
\hline 0 & $u_{0}=\left[\begin{array}{llll}1 & -1 & -1 & -1\end{array}\right]^{T}$ & $W_{0}^{\{1\}}$ & $W_{0}^{\{14\}} / \sqrt{2}$ & $W_{0}^{\{124\}} / \sqrt{3}$ & $W_{0}^{\{1234\}} / 2$ \\
\hline 1 & $u_{1}=\left[\begin{array}{llll}1 & -j & 1 & j\end{array}\right]^{T}$ & $W_{1}^{\{1\}}$ & $W_{1}^{\{12\}} / \sqrt{2}$ & $W_{1}^{\{123\}} / \sqrt{3}$ & $W_{1}^{\{1234\}} / 2$ \\
\hline 2 & $u_{2}=\left[\begin{array}{llll}1 & 1 & -1 & 1\end{array}\right]^{T}$ & $W_{2}^{\{1\}}$ & $W_{2}^{\{12\}} / \sqrt{2}$ & $W_{2}^{\{123\}} / \sqrt{3}$ & $W_{2}^{\{3214\}} / 2$ \\
\hline 3 & $u_{3}=\left[\begin{array}{llll}1 & j & 1 & -j\end{array}\right]^{T}$ & $W_{3}^{\{1\}}$ & $W_{3}^{\{12\}} / \sqrt{2}$ & $W_{3}^{\{123\}} / \sqrt{3}$ & $W_{3}^{\{3214\}} / 2$ \\
\hline 4 & $u_{4}=\left[\begin{array}{llll}1 & (-1-j) / \sqrt{2} & -j & (1-j) / \sqrt{2}\end{array}\right]^{T}$ & $W_{4}^{\{1\}}$ & $W_{4}^{\{14\}} / \sqrt{2}$ & $W_{4}^{\{124\}} / \sqrt{3}$ & $W_{4}^{\{1234\}} / 2$ \\
\hline 5 & 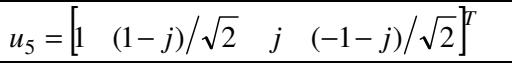 & $W_{5}^{\{1\}}$ & $W_{5}^{\{14\}} / \sqrt{2}$ & $W_{5}^{\{124\}} / \sqrt{3}$ & $W_{5}^{\{1234\}} / 2$ \\
\hline 6 & 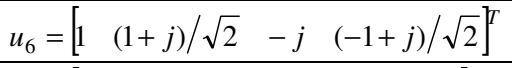 & $W_{6}^{\{1\}}$ & $W_{6}^{\{13\}} / \sqrt{2}$ & $W_{6}^{\{134\}} / \sqrt{3}$ & $W_{6}^{\{1324\}} / 2$ \\
\hline 7 & 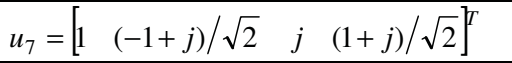 & $W_{7}^{\{1\}}$ & $W_{7}^{\{13\}} / \sqrt{2}$ & $W_{7}^{\{134\}} / \sqrt{3}$ & $W_{7}^{\{1324\}} / 2$ \\
\hline 8 & $u_{8}=\left[\begin{array}{llll}1 & -1 & 1 & 1\end{array}\right]^{T}$ & $W_{8}^{\{1\}}$ & $W_{8}^{\{12\}} / \sqrt{2}$ & $W_{8}^{\{124\}} / \sqrt{3}$ & $W_{8}^{\{1234\}} / 2$ \\
\hline 9 & $u_{9}=\left[\begin{array}{llll}1 & -j & -1 & -j\end{array}\right]^{T}$ & $W_{9}^{\{1\}}$ & $W_{9}^{\{14\}} / \sqrt{2}$ & $W_{9}^{\{134\}} / \sqrt{3}$ & $W_{9}^{\{1234\}} / 2$ \\
\hline 10 & $u_{10}=\left[\begin{array}{llll}1 & 1 & 1 & -1\end{array}\right]^{T}$ & $W_{10}^{\{1\}}$ & $W_{10}^{\{13\}} / \sqrt{2}$ & $W_{10}^{\{123\}} / \sqrt{3}$ & $W_{10}^{\{1324\}} / 2$ \\
\hline 11 & $u_{11}=\left[\begin{array}{llll}1 & j & -1 & j\end{array}\right]^{T}$ & $W_{11}^{\{1\}}$ & $W_{11}^{\{13\}} / \sqrt{2}$ & $W_{11}^{\{134\}} / \sqrt{3}$ & $W_{11}^{\{1324\}} / 2$ \\
\hline 12 & $u_{12}=\left[\begin{array}{llll}1 & -1 & -1 & 1\end{array}\right]^{T}$ & $W_{12}^{\{1\}}$ & $W_{12}^{\{12\}} / \sqrt{2}$ & $W_{12}^{\{123\}} / \sqrt{3}$ & $W_{12}^{\{1234\}} / 2$ \\
\hline 13 & $u_{13}=\left[\begin{array}{llll}1 & -1 & 1 & -1\end{array}\right]^{T}$ & $W_{13}^{\{1\}}$ & $W_{13}^{\{13\}} / \sqrt{2}$ & $W_{13}^{\{123\}} / \sqrt{3}$ & $W_{13}^{\{1324\}} / 2$ \\
\hline 14 & $u_{14}=\left[\begin{array}{llll}1 & 1 & -1 & -1\end{array}\right]^{T}$ & $W_{14}^{\{1\}}$ & $W_{14}^{\{13\}} / \sqrt{2}$ & $W_{14}^{\{123\}} / \sqrt{3}$ & $W_{14}^{\{3214\}} / 2$ \\
\hline 15 & $u_{15}=\left[\begin{array}{llll}1 & 1 & 1 & 1\end{array}\right]^{T}$ & $W_{15}^{\{1\}}$ & $W_{15}^{\{12\}} / \sqrt{2}$ & $W_{15}^{\{123\}} / \sqrt{3}$ & $W_{15}^{\{1234\}} / 2$ \\
\hline
\end{tabular}


Advantages of open-loop spatial multiplexing over closed-loop spatial multiplexing:

- Lower feedback overhead on the uplink channels without PMI feedback.

- May offer better downlink performance at medium or high velocities where the PMI can be unreliable.

- Lower UE and eNodeB implementation complexity since the applied precoder is fixed or cyclic.

\subsubsection{TM4 Closed-Loop Spatial Multiplexing}

TM4 is known as closed-loop spatial multiplexing (CLSM) due to the closed-loop control system that reports optimal precoding matrix choices to the eNodeB to improve match the eNodeB transmission to the channel and improve the throughput. TM4 has similarities to TM3 (see section 2.5.3). TM4 uses the CQI and RI to adapt the MCS, number of codewords and layers for transmission in the same fashion as TM3. However, unlike open-loop spatial multiplexing in TM3, TM4's closed-loop spatial multiplexing scheme attempts to match the channel by reporting the UE-preferred precoding matrix to the eNodeB, using the PMI. The knowledge of the UE-preferred precoder at the eNodeB gives TM4 an edge over TM3 in low velocity scenarios where the precoder information is reliable. The knowledge of the optimal precoding matrix at the eNodeB in TM4's closed-loop spatial multiplexing scheme permits the eNodeB to better match the channel and transmit at higher bit rates than TM3.

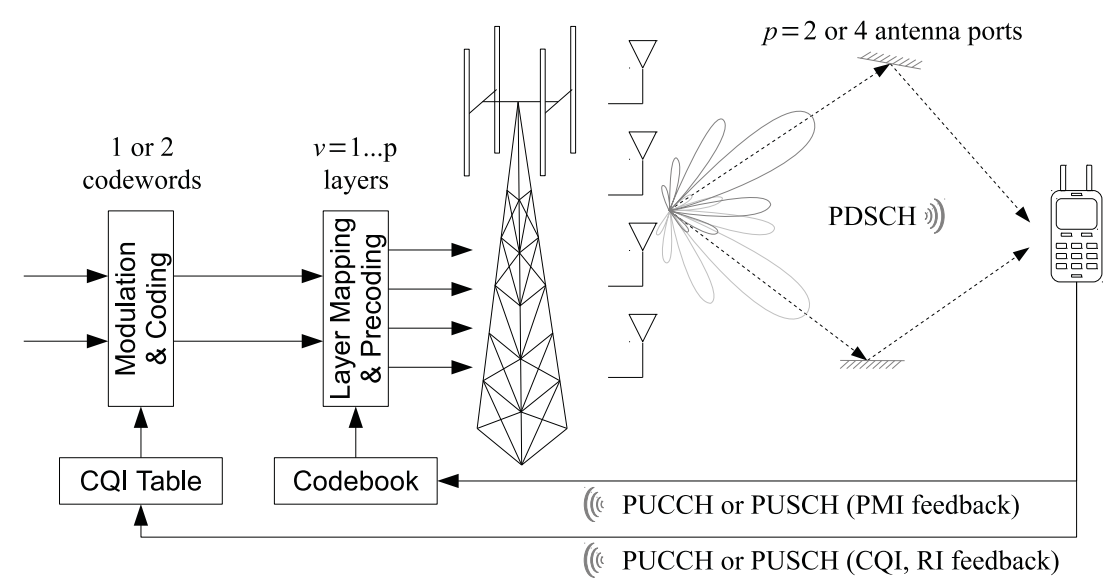

Figure 2.42: Closed-Loop Spatial Multiplexing (CLSM) 
In contrast to TM3, TM4 does not make use of CDD, a transmission scheme described in section 2.5.3 that is used to overcome frequency flat channels. Since TM4 tries to exploit the MIMO channel for spatial multiplexing purposes by trying to accurately adapt the transmit and receive beamforming to the channel, it would be counter productive to add CDD to TM4. The purposes of each mode becomes apparent as TM4 is more dependent on the channel then TM3. Therefore, TM4's CLSM scheme is expected to be used in low-medium velocity scenarios where the UE can report an accurate precoder for transmit beamforming and TM3's OLSM scheme is intended for lower complexity implementations and medium-high velocity where TM4's performance may be degraded.

\section{Downlink Signaling in TM4}

TM4 is split into two modes of operation where the highest throughput is achieved by closed-loop spatial multiplexing (CLSM) transmission scheme. As in TM3, TM4 also uses transmit diversity as a robust fall-back mode. The eNodeB determines the transmission scheme based on the channel state information reported by the UE after the UE performs channel measurements and analysis. When the PDSCH is transmit on the downlink by the eNodeB, the DCI format used in the control channel indicates to the UE which transmission scheme was used to transmit the PDSCH. The DCI format for closed-loop spatial multiplexing and transmit diversity for TM4 are shown in table 2.18.

Table 2.18: TM4 PDSCH Transmission Scheme

\begin{tabular}{cccc}
\hline Mode & DCI Format & Search Space & Transmission Scheme \\
\hline \hline \multirow{2}{*}{ TM4 } & DCI 1A & CRS \& UE specific by C-RNTI & Transmit diversity \\
\cline { 2 - 4 } & DCI 2 & UE specific by C-RNTI & CLSM or Transmit diversity \\
\hline
\end{tabular}

The UE learns of the PDSCH transmission by the detection of a PDCCH CRC scrambled with the UE's assigned C-RNTI. The UE is informed of the PDSCH transmission scheme by determining the DCI format sent in the PDCCH. The two possible DCI formats indicate to the UE whether TM4's closed-loop spatial multiplexing transmission scheme or transmit diversity was used to transmit the PDSCH in the same sub-frame. 


\section{Link Adaptation in TM4}

Link adaptation in TM4 is similar to that of TM3 with respect to the CQI and RI. Both channel state indicators are reported to the eNodeB as in TM3. The eNodeB can configure the CQI to be sent periodically or a-periodically on a wideband or sub-band basis and the RI determines the number of layers and codewords. LTE's frequency selective scheduling is also possible in TM4 using the sub-band CQI values reported by the UE. FSS enables the eNodeB to schedule the connected UEs in portions of the channel that offer the best channel conditions to maximize the use of the downlink channel resources.

The difference between closed-loop spatial multiplexing in TM4 and open-loop spatial multiplexing in TM3 is the optimal precoding matrix report that is sent from the UE to the eNodeB. The additional precoding information increases the complexity of the link adaptation algorithm at the UE but can also improve the downlink throughput.

\section{Precoding in TM4}

In TM4, the link adaptation control loop includes a precoding matrix indicator (PMI) in the channel state information reported to the eNodeB via the uplink. The UE-preferred precoder is applied to the PDSCH transmissions at the base station using the precoder matrix $\boldsymbol{W}(\boldsymbol{i})$. The complex layer-mapped symbols $\boldsymbol{x}$ are transformed by the precoder $\boldsymbol{W}(\boldsymbol{i})$ and transmit using equation 2.58 found in section 2.5.3. The application of the optimal precoder to the eNodeB transmissions maximizes the capacity of the channel by matching the precoder with the largest singular values of the channel as shown in figure 2.11 and discussed in section 2.3.3. The result is a diagonal channel with independent parallel data streams that can be used to transmit multiple codewords if the rank is greater than one. As the channel varies over time, the rank and precoder and MCS are periodically adapted to take advantage of the available data streams found by channel analysis.

The optimal precoder selection at the UE adds a third dimension to the link adaptation algorithm that resides in the UE and attempts to maximize the use of the downlink. The additional dimension adds a significant amount of complexity in some conditions such as when multiple codewords and a large set of sub-band precoders and CQIs are possible. Therefore, to reduce the complexity the number of precoding matrices are limited to ensure that the link adaptation algorithms are practical even for low cost UE implementations. The size of the set of precoders is also limited by the amount of overhead control bits that can be used for PMI reporting on the uplink. The overhead, link adaptation algorithm complexity and the uniqueness and quality of the beam patterns are trade-offs that were considered in 
the design of codebooks used by the spatial multiplexing transmission modes in LTE.

The UE reports its desired precoder using a precoding matrix indicator (PMI) value that points to the precoder within the same codebooks discussed in section 2.5.3. The precoder codebook for closed-loop spatial multiplexing using two antenna ports is given in table 2.16, while the precoder codebook for four antenna ports is given in table 2.17.

The selection of the precoder at the UE is part of the channel resource maximizing problem that will be discussed in section 2.5.4. The optimization task is left up to the UE since the UE performs the downlink channel measurement based on the CRS and it would be inefficient to transmit the raw channel measurements back to the eNodeB. The maximization problem is more complex in TM4 than in TM3 due to the need to find the optimal rank $L$, precoders $\hat{\boldsymbol{W}}_{s}$ and AMC schemes $\hat{\boldsymbol{m}}_{s}$ for each sub-band $s$ of each codeword $c$. A method to select the link adaptation parameters is proposed in [27] and [28] and is briefly described in section 2.5.4 to highlight the differences between TM3 and TM4 and the strengths and possible weaknesses of TM4's closed-loop spatial multiplexing transmission scheme.

\section{Optimal Channel State Indicator Selection in TM4}

The optimal selection of downlink transmission parameters for spatial multiplexing transmission modes is the step that occurs after channel measurements, analysis, equalization and other impairment compensation, but before the link adaptation parameters are transmit to the eNodeB on one of the uplink channels. In this section, a method to select the spatial multiplexing transmission parameters is discussed to provide the reader with an understanding of the PMI and CQI selection process and the added level of complexity that is inherently part of closed-loop spatial multiplexing.

The MIESM based maximization problem presented in [29] chooses the set of feedback parameters (PMI, CQI and RI) that maximizes the overall spectral efficiency of the LTE downlink. In the maximization problem presented in equation 2.62, the spectral efficiency $E_{s}^{c}$ of each sub-band $s$ and each codeword $c$ is summed for a set of precoders $\hat{\boldsymbol{W}}_{s}$ and AMC schemes $\boldsymbol{m}_{s}[c]$. The set of feedback parameters (PMI, CQI and RI) that result in the highest spectral efficiency is sent to the eNodeB to be used for future downlink transmissions.

$$
\left[\hat{L},\left\{\hat{\boldsymbol{W}}_{s}\right\}_{S},\{\hat{\boldsymbol{m}}\}_{S}\right]=\arg \max \sum_{s=1}^{S} \sum_{c=1}^{C_{L}} E_{s}^{c}\left(\boldsymbol{W}_{s}, \boldsymbol{m}_{S}[c]\right)
$$

In equation 2.62, the optimal rank $\hat{L}$, set of optimal precoders $\left\{\hat{\boldsymbol{W}}_{s}\right\}_{S}$ and set of optimal MCSs $\{\hat{\boldsymbol{m}}\}_{S}$ that maximize the total spectral efficiency across the $S$ sub-bands and $C_{L}$ 
codewords available for transmission are reported to the eNodeB for subsequent spatial multiplexing transmissions.

For optimal results, an exhaustive search performed over all the possible feedback parameters would be required to solve the maximization problem presented in equation 2.62. In reality, the complexity of an algorithm that performs an exhaustive search over all the spatial multiplexing parameters that can be varied in TM4 is high. In order to meet the maximum processing delays required by the LTE standards, a link adaptation algorithm with a lower complexity is needed.

An example of a lower complexity algorithm is proposed in [28]. The lower complexity algorithm proposed by the authors in [28] splits the maximization problem into sequential steps where the goal of the lower complexity remains the same but can be performed within the constraints imposed by technology used in the system and the LTE standards. The two step algorithm also tries to maximize the total spectral efficiency, as shown in equation 2.62 which calculates and selects the link adaptation parameters that lead to the highest total spectral efficiency $E_{s}^{c}$ across all available resources.

The first step is based on the work previously published in [27]. In [27], the authors present a low complexity method that can be used to select precoders for each sub-band using the post equalization SNR of the sub-carriers and the available precoders in codebook $\mathcal{W}$. The UE first determines the precoder that maximizes the channel capacity in the sub-band(s) of interest by finding the precoder that maximizes the mutual information $I_{k, l}$ over a time-frequency range of interest. To keep the complexity of the precoder optimization problem low, the authors suggest the use of the same channel matrix ${ }^{10}$ in the calculation of the mutual information $I_{k, l}$ across an LTE resource block is reasonable since simulations performed in [27] showed that it is possible to use the same channel matrix for optimal precoder selection without suffering significant spectral efficiency losses across small bandwidths.

The precoder maximization problem described in [27] is shown in equation 2.63. The mutual information $I_{k, l}$ for resource element $(k, l)$ is a function of the SNR of the subcarriers being considered and is based on the channel measurement. For more information on the calculation of the mutual information the reader is referred to [27] since the details are beyond the scope of the thesis. The result of the maximization problem is a single precoder $\boldsymbol{W}_{j}$ per sub-band that maximizes the sub-band channel capacity. The precoders are

\footnotetext{
${ }^{10}$ The channel matrix used for optimal precoder selection can be found using channel sub-sampling by taking the channel at the mid-point of the sub-band of interest when the sub-bands width are small and complexity needs to be low or by channel averaging when the sub-band widths are large.
} 
carried forward and used in the second step of the algorithm which attempts to determine the optimal sub-band CQIs.

$$
\boldsymbol{W}_{j}=\underset{\boldsymbol{W}_{i} \in \mathcal{W}}{\arg \max } \sum_{k} \sum_{l} I_{k, l}\left(\boldsymbol{W}_{i}\right)
$$

The second step of the algorithm presented in [28] uses the optimal precoder $\boldsymbol{W}_{j}$ from the first step to find the highest CQI values for each sub-band that satisfy the $10 \%$ physical layer BLER limit and simultaneously maximize the spectral efficiency across the available channel resources.

The UE calculates a single equivalent SNR for each sub-band using the individual SNRs of the sub-carriers within the sub-bands. The SNRs of the resource elements (REs) in the sub-band of interest are mapped to a single average sub-band SNR value using one of two methods. The first method, known as MIESM, is proposed in [28], while, the second method that can be employed is the well known EESM method. For the EESM method, the fading to AWGN channel mapping function is exponential, while the mapping function used by the MIESM method is given by the bit-interleaved coded modulation (BICM) capacity, another topic that is beyond the scope of the thesis but found in [30]. Both the EESM and MIESM SNR mapping methods calculate a single non-fading AWGN channel SNR value that is equivalent to the overall SNR of the sub-band of the actual fading channel measured by the UE. In other words, EESM and MIESM relates the SNR measured in a fading channel to a corresponding AWGN SNR value that achieves the same spectral efficiency as the fading channel.

The calculation of a single MIESM SNR value for each sub-band $s$ and codeword $c$, using the optimal precoder $\boldsymbol{W}_{s}=\boldsymbol{W}_{j}$ determined in the previous step, is accomplished using equation 2.64 and the BICM capacity function $f_{m}(S N R)$. The MIESM SNR is found by summing the mutual information corresponding to the post-equalization SNR of each sub-carrier and then using the inverse BICM capacity function $f_{m}^{-1}(S N R)$ to convert the average mutual information back to an average SNR. The average AWGN SNR found by MIESM in this step represents the original fading channel across the resource elements $r \in \mathcal{R}_{s}$ and transmission layers $l \in \mathcal{L}_{c}$.

$$
S N R_{s}^{c}\left(\boldsymbol{W}_{s}, m\right)=f_{m}^{-1}\left\{\frac{1}{\left|\mathcal{R}_{s}\right|\left|\mathcal{L}_{c}\right|} \sum_{r \in \mathcal{R}_{s}, l \in \mathcal{L}_{c}} f_{m}\left[S N R_{r, l}\left(\boldsymbol{W}_{s}\right)\right]\right\}
$$

The sub-band AWGN SNR $S N R_{s}^{c}$ for codeword $c$ is then used to estimate the BLER, represented by $P_{s}^{c}$, for sub-band $s$, codeword $c$ while considering all possible modulation 
and coding schemes. In equation 2.65, the average AWGN SNRs $S N R_{s}^{c}$ for each sub-band, found using equation 2.64 in the previous step, are mapped to BLER values via the AWGN SNR to BLER mapping table $g_{m}$.

$$
P_{s}^{c}\left(\boldsymbol{W}_{s}, m\right)=g_{m}\left(\operatorname{SNR}_{s}^{c}\left(\boldsymbol{W}_{s}, m\right)\right)
$$

The modulation and coding schemes that satisfy the LTE BLER upper bound of 10\%, represented by $P_{b}^{(t)}$, are kept while the other MCSs are dropped using the thresholding function seen within $h_{m}(P)$. At this stage the MCSs for each sub-band of each codeword that meets the BLER target $P_{b}^{(t)}$ are mapped to a corresponding spectral efficiency value $e_{m}$, as indicated by the operation in 2.66 .

$$
h_{m}(P)= \begin{cases}e_{m} & P_{m} \leq P_{b}^{(t)} \\ 0 & P_{m}>P_{b}^{(t)}\end{cases}
$$

The spectral efficiency for sub-band $s$, codeword $c$ while using MCS $m$ is calculated in equation 2.67. The spectral efficiency corresponds to the spectral efficiency found in equation 2.66 times the block success rate, which is derived from the BLER $P_{s}^{c}\left(\boldsymbol{W}_{s}, m\right)$.

$$
E_{s}^{c}\left(\boldsymbol{W}_{s}, m\right)=h_{m}\left(P_{s}^{c}\left(\boldsymbol{W}_{s}, m\right)\right) \cdot\left(1-P_{s}^{c}\left(\boldsymbol{W}_{s}, m\right)\right)
$$

The total spectral efficiency for all sub-bands and codewords using the modulation and coding schemes are calculated using the spectral efficiencies of each sub-band $s$ and codewords $c$ found using equation 2.67. The efficiencies achieved with each MCS are compared and the MCS that leads to the highest total spectral efficiency over all the sub-bands is selected. The set of link adaptation parameters (MCS, rank and precoder) that maximize the spectral efficiency are converted to their corresponding channel state indicators (CQI, PMI and RI) and are then reported to the eNodeB for future transmissions.

The benefit of using an optimization algorithm based on information theory is that it allows the UE to compare the spectral efficiency of the transmission settings in order to make the best use of the channel between the UE and the eNodeB and transmit at the highest possible information-theoretic rate. When each UE is making the best use of its channel the consumer experience is improved due to higher throughputs, lower latencies and lower mobile device battery drain. Lower service costs are also possible since the operator's spectral resources can be shared more efficiently between a greater number of users. 
Based on the above, it is clear that the optimal precoder selection and reporting that occurs in closed-loop spatial multiplexing offers the link adaptation control loop more degrees of freedom to try to match the channel then previously discussed transmission modes (TM1, TM2, TM3). TM4 has the potential for high spectral efficiency under favourable channel conditions. This is increasingly true as the number of antenna elements and logical antenna ports are increased from two to four since the number of available precoders increases from four to sixteen and the potential for system throughput performance increases.

On the other hand, if TM4 is used and significant impairments are introduced that affect the link adaptation control system, such as processing delays, channel measurement errors, rapid channel fluctuations where the UE reported information quickly becomes aged, TM4's greater dependence the link adaptation control system could cause the downlink throughput to deteriorate. This will become apparent in section 4 of this thesis where a number of impairments that can affect the performance of the various transmission modes and their link adaptation systems are simulated.

\subsubsection{TM6 Single Layer Closed-Loop Spatial Multiplexing}

TM6 is known as closed-loop spatial multiplexing using only a single transmission layer. The operation of TM6 is similar to TM4 since both modes use closed-loop spatial multiplexing. The difference between the two modes is the single transmission layer limit which is imposed on TM6. Since TM6 can only use a single transmission layer, it is also limited to a single codeword, which implies that the single codeword is transmit simultaneously on all available antenna ports after precoding. The link adaptation system is only capable of varying the MCS and precoder, effectively reducing the system complexity and making the main purpose of TM6 "single layer closed-loop beamforming".

TM6 is briefly mentioned here since its performance should be equivalent to TM4 with the rank fixed to one. TM4 rank-1 is used in section 4.1.5 and section 4.2 of chapter 4 of this thesis to compare the fall-back mode TM2 with a spatial multiplexing mode. In order to perform a fair comparison between TM2 and TM4, the selected spatial multiplexing transmission mode, the rank of TM4 is fixed to one since TM2 is only capable of transmitting one codeword. Finally, the use of TM4 with its rank fixed to one is equivalent to using the sixth transmission mode, known as TM6. 


\section{Link Adaptation and Precoding in TM6}

TM6 permits the adaptation of the CQI and the PMI as in TM3 and TM4 but always uses a single codeword and single transmission layer for its downlink transmissions. The CQI and PMI are reported on one of the uplink channel as illustrated in figure 2.43.

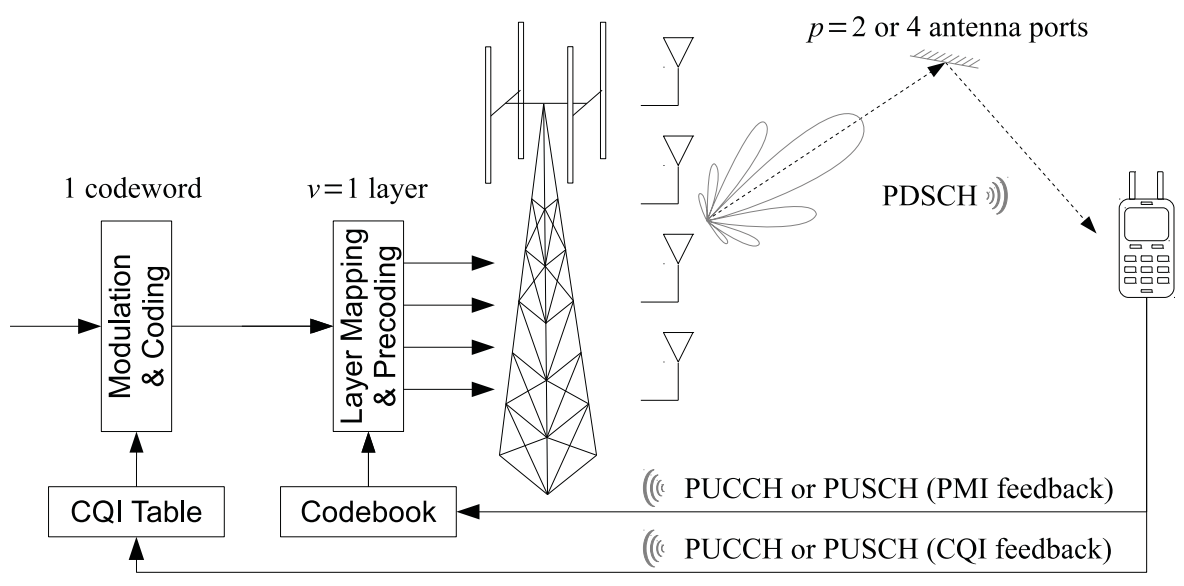

Figure 2.43: Single-Layer Closed-Loop Spatial Multiplexing (Codebook Based Beamforming)

The link adaptation system in TM6 permits the transmission rate to be adapted to the allocated spectral resources as in previous modes, using the CQI. If sub-band reporting is enabled, frequency selective scheduling can take place at the eNodeB such as in other transmission modes.

The precoding stage in the transmission chain is also adapted. TM6 is also referred to as codebook based beamforming or closed-loop rank-1 beamforming since beamforming is the primary purpose of the mode since it is fixed to a single transmission layer and it is dependent on the codebooks defined in LTE physical layer standard [1]. The benefit of closed-loop rank-1 beamforming is that it is a robust transmission scheme at low SNR where single layer transmissions succeed more often then multi-layer transmissions as long as the feedback for transmit beamforming is still reliable at the time of the next PDSCH transmission. 


\section{Chapter 3}

\section{Performance Simulation Setup}

\subsection{Link Level Simulator}

Simulation results presented in this thesis were performed using a proprietary link level simulator developed at Ericsson. The simulator is categorized as a link level simulator due to the detailed implementation of the LTE PHY and MAC layers and the advanced MIMO channel models that are used to model the data link layer performance in a wireless environment. Ericsson's simulator is used extensively in chapter 4 of this thesis to obtain throughput performance results for the LTE data link layer in wireless environments modeled by the spatial channel model and the 3GPP EPA conformance testing channel model.

\subsubsection{Downlink Channel Modeling in the Link Level Simulator}

The link level simulator takes a number of user inputs to appropriately configure the LTE transmission settings, transmitter and receiver modeling parameters and the channel modeling parameters. A high level block diagram of the simulator, the transmitter (discussed in section 2.4.1), the receiver, the downlink channel model, the ideal uplink channel for feedback reporting and a list of input parameters are shown in figure 3.1. The simulator input parameters are discussed in greater detail in section 3.4.

In the downlink simulations run in chapter 4 , the base station is configured as the transmitter, and a single UE as the receiver. The downlink is transmit from the base station antenna ports to the UE's receiving antennas. The LTE transmission parameters are used to configure the OFDM transmissions as well as scheduling and link adaptation, while the channel modeling parameters are used to subject the UE to the desired wireless environment and position the base station and UE within the environment. Finally, when 


\section{Simulator Inputs}

LTE Transmission Settings

\begin{tabular}{|l|}
\hline Transmission Settings \\
$\circ$ Carrier frequency $\left(f_{c}\right)$ \\
$\circ$ Bandwidth $\left(N_{P R B}^{B W}\right)$ \\
$\circ$ Dupplex mode (FDD or TDD) \\
$\circ$ OFDM sub-carrier spacing $(\Delta f)$ \\
$\circ$ Cyclic prefix duration $\left(T_{C P}\right)$ \\
$\circ$ Number of sub-frames (or OFDM \\
symbols) to simulate \\
$\circ$ Transmission mode \\
$\circ$ Antenna ports \\
$\circ$ Reference signal power boosting \\
$\circ$ PDCCH control symbols \\
$\circ$ HARQ and OLLA \\
Scheduling \\
$\circ$ Number of PRB allocated to UE \\
CSI reporting mode, interval \& delay \\
$\circ$ CQI and PMI reporting group sizes \\
\hline
\end{tabular}

Transmitter \& Receiver Modeling Parameters Channel Analysis Algorithms - Time/freq synchronization - Channel estimation Impairments

- Base station impairments - BS transmitter phase noise - UE impairments

- Receiver phase noise - IQ imbalance
Channel Modeling Parameters

- Base station

- Location, orientation

- Antenna array height $\left(h_{b s}\right) \&$ orientation

- \# of elements, spacing and polarization

- User Equipment

- Location, orientation

- Antenna array height $\left(h_{m s}\right) \&$ orientation - \# of elements, spacing \& polarization

Channel

-SNR

-UE velocity

- EPA channel model

- Antenna MIMO correlation matrix

- SCME channel model

- Scenario

- Mean angle spread (AS) at the BS

- Cross-polarization discrimination ratio

\section{Link Level Simulator}

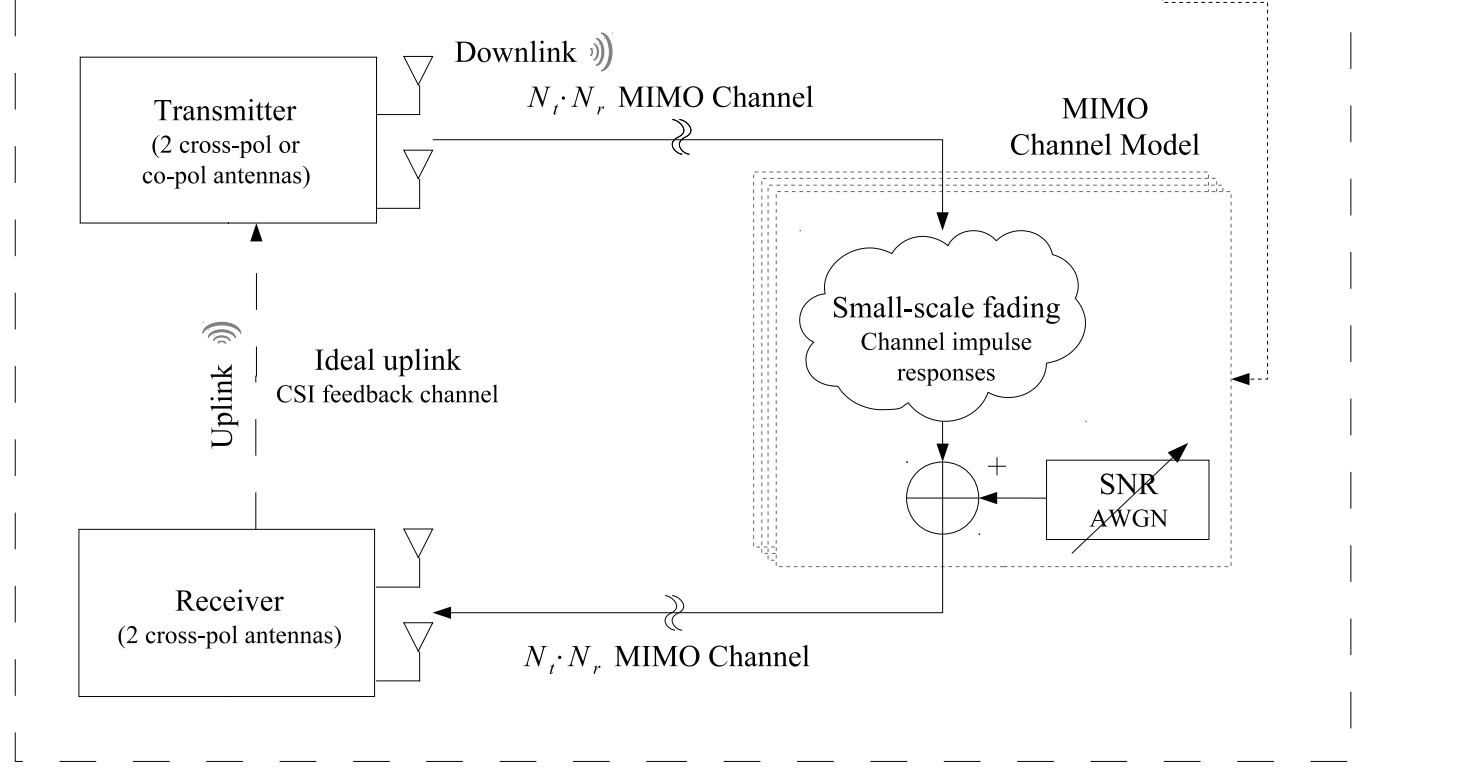

Figure 3.1: User Input Parameters for the Link Level Simulator 
applicable, the transmitter and receiver modeling parameters are used to set the receiver algorithms and add impairments to model the imperfections of the RF front-ends in the transmitter and receiver.

A number of the simulations presented in chapter 4 are based on the SCME, while some comparisons are made with the EPA channel model. A primary difference between the SCME and the EPA channel model is better modeling of the channel's spatial characteristics in the SCME. To better model the channel characteristics, the SCME requires a description of the cell layout, which includes the BS and UE locations and orientations, and a physical description of the base station and UE antenna arrays. The cell layout and antenna array physical descriptions are required by the SCME to better model the characteristics of the sub-paths departing the base station and arriving at the UE.

Aspects of the SCME that depend on the cell layout and antenna array description:

- Large-scale (path loss) effects: Uses the distance between the UE and the eNodeB.

- Antenna element correlation: Uses the spacing between the array elements.

- Sub-path gains: Depend on the orientation of the antenna array, antenna element field pattern and the randomly drawn angles of departure and arrival.

- Antenna polarization: Uses the orientation of the antenna elements.

For the SCME, the simulator passes the cell layout and antenna array description to the spatial channel model which uses the geometry to generate the channel realization $h_{u, s, n}(t)$ at time step $t$, for each link between the BS and the UE forming the MIMO channel matrix, as described in section 2.3.8.

In the case of the EPA simulations, the $3 \mathrm{GPP}$ conformance testing model does not use geometry and antenna array descriptions since the simulator is of lower complexity and does not model as many spatial characteristics as accurately as the SCME. Instead, the taps of the power delay profile of the EPA channel model are predefined in the 3GPP LTE standards to model the outdoor-to-indoor pedestrian environment with low delay spread (extended pedestrian model "A"). The average tap powers and tap delays of the channel impulse response are predefined in the appendices of [6] and [4] based on the ITU-R recommendation M.1225 [31], along with the MIMO channel correlation matrices used for EPA simulations. 


\section{The Cell Layout (SCME Only)}

Geometry is provided to the SCME simulator in order to better model the spatial aspects of the channel wireless channel. The precise position, orientation and tilt of the BS and the UE can be used to evaluate the performance of algorithms, transmission schemes and beamforming at particular locations and orientations of interest in the wireless environment. In this thesis, the network layout was used to specify the location and orientation of the base station and the UE when SCME simulations were run.

The network layout for the SCME simulations is shown in figure 3.2. A single serving cell is used and centered at the origin $(x, y)=(0,0)$. The base of the serving base station is placed at the center of the serving cell at coordinate $(x, y)=(0,0)$ meters.

In the simulations in chapter 4 as single UE is used to measure the downlink throughput. The UE is located at 550 meters from the base station at an angle of $15^{\circ}$ from the $\mathrm{x}$-axis, or more specifically $(x, y)=\left(550 \cos 15^{\circ}, 550 \sin 15^{\circ}\right)$ meters, as shown in figure 3.2.

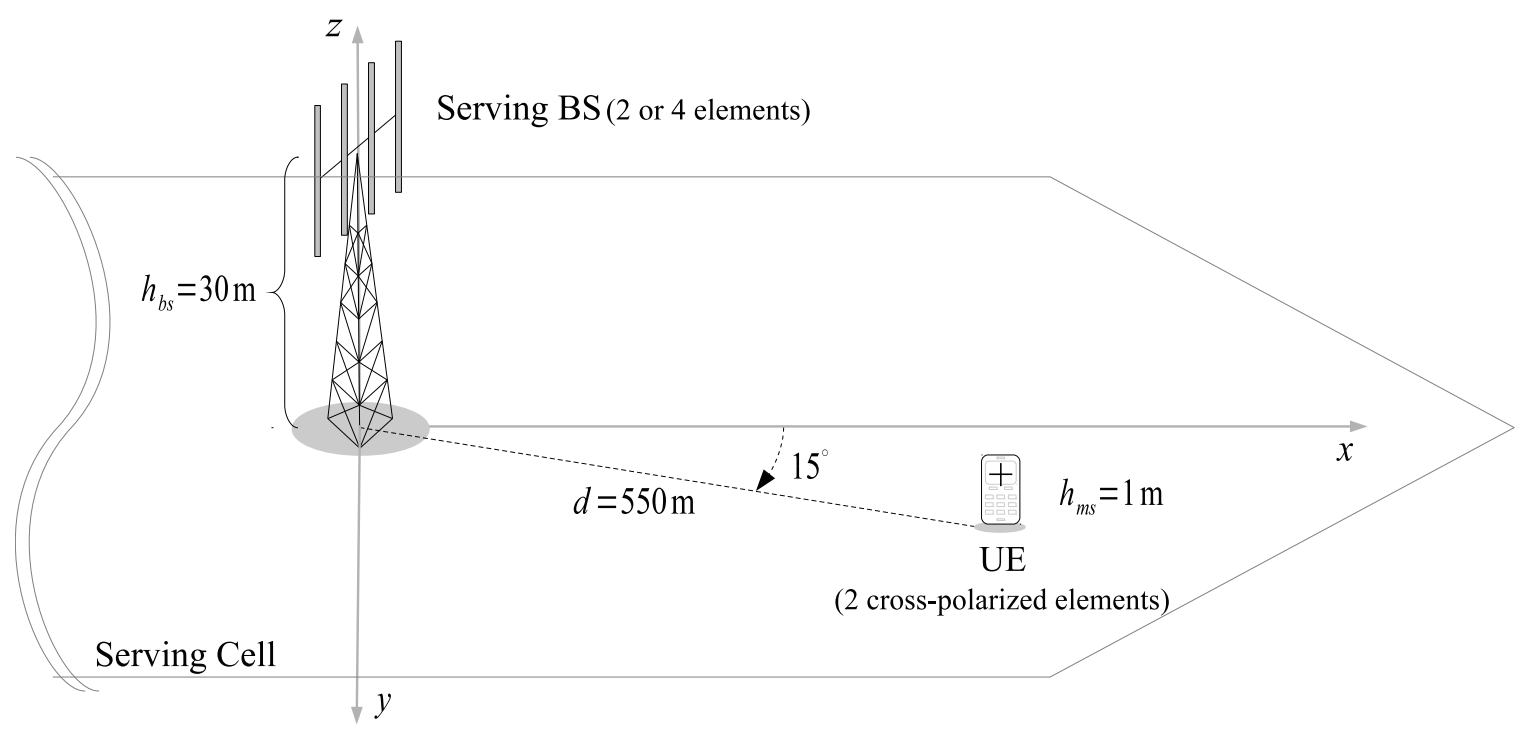

Figure 3.2: The Simulation Scenario used with the Extended Spatial Channel Model (SCME)

\section{The Base Station Antenna Array}

The base station antenna array is placed at an elevation of 30 meters above ground level to represent a practical base station, placing the BS antenna array at the coordinate $(x, y, z)=(0,0,30)$ meters. The orientation of the base station is such that the base station 
antenna array boresight parallels the $\mathrm{x}$-axis. The antenna array configuration, including the polarization and spacing, depends on the simulation goals of the particular study performed in chapter 4 . In chapter 4 , the quad base station antenna package is compared to the two antenna package with cross-polarized and co-polarized antennas, where the cross-polarized antennas are mounted at angles of $-45^{\circ}$ and $+45^{\circ}$. The correlation is set to either low correlation or high correlation using the antenna element spacings $10 \lambda$ or $0.7 \lambda$ when using the SCME, or low or high correlation matrices when using the conformance testing model EPA. The base station antenna arrays used in this thesis are depicted in figure 4.1 for the co-polarized antenna and figure 4.2 for the cross-polarized antennas.

\section{UE Antenna Array}

The UE antenna array is elevated by 1 meter above ground level to approximate a pedestrian scenario and places the antenna array at $(x, y, z)=\left(550 \cos 15^{\circ}, 550 \sin 15^{\circ}, 1\right)$ meters. Two antennas are used at the UE due to the desire to integrate multiple antennas to transmit multiple codewords. Two antennas were chosen since the available space in typical cellular and smart phone devices is limited and, to be effective, the antenna elements should receive uncorrelated signals. Therefore, two cross-polarized elements were used at the UE. An edipole and $\mathrm{m}$-dipole is used to model the UE antenna elements with element polarizations of $-90^{\circ}$ and $90^{\circ}$. The UE is shown in figure 4.3a.

\section{The Drop for SCME Simulations (SCME Only)}

A drop is composed of the physical location and description of the BS and the UE and the randomly generated scatterers (or clusters) that describe the wireless environment. The wireless environment is modeled using the ray model (see figure 2.18 in section 2.3.7), the scenario, the mean angle spread if applicable, and the environment parameters that are generated by the channel model based on the scenario selected. In the ray model, the $N=6$ paths are composed of $M=20$ sub-paths per path between the base station and the UE. The paths in the ray model are described by a number of distributions defined in the environment parameters that are used to determine the angular parameters, the randomly drawn sub-path phases, path delays and path powers and antenna field patterns gains associated with the sub-path angles of departure, as explained in section 2.3.7.

In this thesis, the locations, orientations and physical descriptions of the antennas of the base station and UE are taken from the fixed network layout, described earlier, as an input to the simulator. The angular parameters and other path characteristics in the drop are 
randomly generated by the channel model based on the network layout and the environment parameters selected by the scenario and mean angle spread. The scatterers remain fixed after being generated but the channel experiences fading at the Doppler rate provided by the user, by the $U E$ velocity input, in meters per second. A disadvantage of fixing the UE location and re-using the same drop, or in other words the same channel realization, is that the results are specific to the drop generated by the channel model and the random number generator seed.

The reason the location of the entities and the channel seed are fixed during all of the simulations in this thesis is to compare the effect of different LTE transmission settings and configurations. The network layout and drop was kept the same to allow for the fair comparison while varying the input parameters listed below.

Input parameters varied for performance evaluation in this thesis:

- Antenna related:

- Number of base station elements (2 or 4)

- Number of base station antenna ports

- Base station array element spacing

- Transmission and link adaptation settings:

- Transmission mode

- UE processing delay

- UE channel state reporting interval

- Impairments:

- Amount of phase noise and type

- UE velocity

- Signal-to-noise ratio

The results of the SCME performance simulations in chapter 4 are specific to the fixed network layout and fixed drop since a single channel seed was used to compare the performance of the transmission modes and antenna configurations. If other UE locations were chosen, or other drops were simulated, it is possible that the channel conditions become favourable to another transmission mode or antenna configuration. 


\section{Base Station Implementation}

The link level simulator models the base station by transmitting randomly generated data bits on a simulated physical downlink shared channel using the antenna setup, transmission mode and impairments in each study in chapter 4.

The LTE link level simulator transmits bits from the base station by modeling the complete LTE transmission chain, including code block segmentation, channel coding using the turbo encoder/decoder, scrambling, modulation and resource mapping, IFFT and CP addition, as described in section 2.4.1. The OFDM baseband time domain samples are transmit across the available antenna ports based on the transmission mode set by the user and the UE reported channel state indicators. The link adaptation algorithms discussed in sections 2.5.2 to section 2.5.5 are configured for sub-band or wideband reporting depending on the particular study and is noted in the simulation setup section of each of the simulations performed in chapter 4.

In the majority of the performance simulations in chapter 4, outer-loop link adaptation (OLLA) is enabled. OLLA is implemented at the base station and adapts the MCS based on the acknowledgement (ACK) and negative acknowledgment (NACK) reported by HARQ. OLLA is disabled in the simulations in section 4.3 in order to observe the performance of link adaptation and the effect of particular link adaptation parameters without interference from OLLA adjustments.

Finally, the time domain samples for each link are convolved with the channel realizations for each link in the MIMO channel and the desired signal-to-noise ratio is obtained by adding white Gaussian noise.

\section{Receiver Implementation}

The simulator implements a typical UE model in order to decode the transmitted information and gather statistics about the downlink transmissions or the dynamic system state or the reported parameters of interest. The receiver and wireless channel depend on the characteristics of the UE antenna array, impairments applied to the receiver and algorithms used for channel estimation, synchronization and decoding.

The UE implementation receives and decodes the bits after convolving the downlink transmission with the channel realizations generated by the user selected channel model. The bits are decoded at the UE after synchronization, FFT, channel estimation and equalization, resource de-mapping, demodulation and de-scrambling. After decoding, if a transport block CRC fails the transport block can be re-transmit up to 3 times by the eNodeB 
using HARQ. The successfully received blocks are counted toward measurements, such as the throughput measurement used in this thesis.

After channel analysis, the receiver is responsible to report the channel state indicators to the eNodeB using sub-band or wideband reporting as noted in the simulation setup sections in each simulation in chapter 4. An ideal uplink channel is implemented in the Ericsson link level simulator in order to complete the link adaptation loop. The channel state indicators are sent to the eNodeB implementation, which uses this new information to schedule the UE in a set of new resource blocks, if sub-band reporting is enabled for frequency selective scheduling.

\section{Modeling Impairments}

In addition to transmitter and receiver algorithm modeling, the simulator includes the ability to model several impairments that affect the performance of the LTE downlink. The simulator algorithms including impairments are updated on an on-going basis in order to maintain a close relationship between the predicted simulator results and the actual results that would achieved by data link layer downlink transmissions of an Ericsson base station and typical UE. This ensures the results are as accurately describe the performance of a highly complex and practical implementation of base station product. For example, one such impairment is the receiver local oscillator (LO) phase noise and receiver in-phase and quadrature (I-Q) imbalance which is often used to obtain the results in this thesis. Transmitter phase noise is also added to the results in section 4.2. A description of the impairments used in each simulation study in chapter 4 is given in section 3.4.

\subsubsection{Channel Models used in the Simulator}

The Ericsson link level simulator includes numerous channel models in the channel modeling library. The two channel models used to obtain the results in this thesis are the extended pedestrian A (EPA) channel model and the extended spatial channel model (SCME), presented in section 2.3.7.

\section{Spatial Channel Model}

The spatial channel model integrated in the Ericsson simulator was implemented based on the 3GPP technical standard 25.996 [3] and the modifications discussed in [17]. The spatial channel model is known as a stochastically controlled geometric channel model since the 
selected scenario defines the distributions that control the simulator. A number of distributions are used to draw random variables that are used to build the channel impulse response. The average path powers, path delays, shadow fading, angles of departure and angles of arrival are all examples of random variables that are drawn to construct the channel impulse response at each simulation step $t$.

In the simulations in this thesis, the scenario was set to urban macro to represent a typical urban environment, described in table 2.2 of section 2.3.7 or table 5.1 of [3]. The urban macro scenario also permits the user to select between two possible mean angle spread values that represent the angular spreading experienced by the paths departing the base station. The mean angular spread parameter is discussed in section 3.4.5. The randomly drawn angular parameters that characterize the ray based model and form the drop used to generate the SCME channel realizations are based on the distributions defined in table 2.2.

\section{Extended Pedestrian A}

The multi-path fading for the EPA channel is modeled using a tapped delay line representing a pedestrian wireless environment with low delay spread (A). The fixed average tap powers and fixed tap delays are defined by the power delay profile given in appendix B.2 of technical standard [4], rather than using the physical coordinates of the base stations and the UEs to model the MIMO channel. The fixed average taps are independently faded and are characterized by a classical Doppler spectrum in the EPA channel model.

When the EPA channel model is used, the performance results are viewed as representing average performance in a typical pedestrian environment due to the lack of the locations of the base station and the UE. The model is based on measurements of typical pedestrian wireless environments and without the knowledge of the network layout the more advanced spatial characteristics cannot be modeled as accurately as the spatial channel model.

Since the antenna element spacing is not available when using the EPA channel model, the simulator uses predefined antenna correlation matrices to establish the correlation between the transmit and receive antenna elements. Examples of $2 \times 2$ correlation matrices that can be used for 2-transmitter, 2-receiver EPA simulations are shown in appendix D.1. The complete set of correlation matrices for $2 \times 2,4 \times 2,4 \times 4$ and $8 \times 2$ antenna configurations is given in the appendices of [6] and [4]. The correlation matrices used in this thesis, when the EPA channel model is used for simulations, are the low and high correlation matrices for the $2 \times 2$ and $4 \times 2$ antenna configurations found in appendix B.2.3 of [6] and appendix B.5.1 of [4]. 


\subsection{Simulating the LTE Downlink using the Link Level Simulator}

The Ericsson simulator was configured to run the desired simulations using the network layout, environment parameters determined by the scenario and settings discussed in previous sections of this chapter. The channel models used to obtain the simulation results in chapter 4, are both the SCME, discussed in section 2.3.7, and the EPA channel model.

In the majority of simulations in chapter 4 the goal was to plot throughput versus SNR. Therefore, the SNR was swept from -10 to 30 or $34 \mathrm{~dB}$, in steps of 1 or $2 \mathrm{~dB}$ and throughput measurements were stored at each step. At each SNR simulation point, the simulator's channel model generates the MIMO channel realization using the specified channel model. During a simulation run for a given SNR point, the channel undergoes fading at the Doppler rate supplied by the user in the form of the UE velocity, in meters per second. The randomly generated data bits are processed by the LTE transmission chain modeled by the eNodeB implementation and transmit by convolving the baseband time domain samples with the channel and adding noise corresponding to the simulated SNR point.

The OFDM symbol transmissions are received at the UE antennas. The UE implementation receives a complete sub-frame (14 OFDM symbols in the time domain) before decoding the transport block since channel estimation is performed on a sub-frame basis and the transport block bits are spread out across groups of PRBs allocated in the frequency domain. The transport blocks are decoded by the UE and the resulting throughput achieved at each SNR point is stored with the SNR and any other statistics or LTE state variables that were of interest during each of the studies in chapter 4. The throughput was calculated as explained in section 3.3.1.

The throughput versus SNR figures in chapter 4 were created by plotting the throughput measurements versus the corresponding SNR.

\subsection{Performance Measurements and Channel Quality In- dicators}

\subsubsection{Throughput Measurement}

Throughput is used as the measure of downlink performance in this thesis since it is of interest to telecommunication equipment manufacturers and operators. Maximizing the 
throughput permits the operator to serve more consumers, lower the cost of service or provide consumers with higher bits rates. The throughput is affected by the transport block size and number of codewords selected for transmission based on the channel conditions and the reported CQI and the RI.

Throughput data was gathered for each SNR point $\gamma$ simulated or velocity $v$ in the UE velocity simulations in section 4.3.2. Throughput was determined by counting the total number of bits received in transport blocks that were successfully decoded at the receiver. After all 15,000 sub-frames were transmit from the eNodeB, the total number of bits received in successfully decoded transport blocks were divided by the total number of sub-frames transmit during the simulation $(15,000)$, then multiplied by the number of subframes transmit per second $(1,000)$ and divided by $10^{6}$ to get the throughput in Megabits per second.

$$
\begin{gathered}
\text { Throughput }(\gamma \text { or } v)=\frac{(\text { Total bits received })(\text { Sub-frames/second })}{(\text { Number of sub-frames transmit })(\text { Bits per Megabit })} \text { Mbps } \\
\text { Throughput }(\gamma \text { or } v)=\frac{(\text { Total bits received })(1000 \text { subframes } / \text { sec })}{(15,000 \text { subframes })\left(10^{6}\right)} \text { Mbps }
\end{gathered}
$$

\subsubsection{CQI and Rank Measurement}

In section 4.3.3, the average CQI and RI are plotted against SNR for 4 velocities (2.1, 124.6, 200, $350 \mathrm{~km} / \mathrm{hr}$ ) to show the change in CQI and RI at high UE velocities. These channel state indicators are extracted from the reports sent by the UE on the ideal simulator uplink to the eNodeB implementation. OLLA was disabled to prevent the eNodeB from overriding the MCS selected by the UE. The CQI and RI statistics were stored with each corresponding SNR evaluated to generate the plots in section 4.3.4.

\section{Average CQI Reported}

The average CQI reported at each SNR $\gamma$ was calculated by accumulating the 4-bit CQI index at each step and dividing the total by the total number of reports at the end of the simulation run. The affect of the reduction of the average CQI reported and used for trans-

mission was observed by comparing the average CQI versus SNR and throughput versus SNR figures. In order to ensure the throughput performance was directly linked to the average CQI reported, OLLA was disabled to prevent outer-loop adjustments to occur at the 
eNodeB.

\section{Reported Rank Distribution}

The rank distribution was calculated by keeping track of the rank 1 and rank 2 transmissions reported by the UE using the RI reported by the UE during the simulation of each SNR point in the simulation run. The distribution of rank 2 transmissions was calculated by dividing the total number of rank 2 transmission by the total number of RI reports that were received by the eNodeB implementation during the simulation at each SNR.

\subsection{Simulation Parameters}

Table 3.1 is a comprehensive list of the simulation parameters used in this thesis. The actual simulation parameters used in each of the three major studies in chapter 4 are chosen from within table 3.1 based on the simulation requirements of the particular study. A table similar to table 3.1 is provided in the simulation setup section of each study in chapter 4 which contains the exact simulation parameters used for the study. The simulation parameters used in this thesis are explained in this section.

\subsubsection{System Parameters}

\section{Carrier Frequency, OFDM Sub-Carrier Spacing and Cyclic Prefix Length}

An LTE carrier frequency of $2.6 \mathrm{GHz}$ was selected due to the intention to deploy LTE systems in the $2.6 \mathrm{GHz}$ and $700 \mathrm{MHz}$ bands worldwide. Based on the LTE standards, a normal cyclic prefix length of $\sim 4.7 \mu \mathrm{sec}$ was used in the downlink simulations along with an OFDM sub-carrier spacing of $15 \mathrm{kHz}$.

\section{Bandwidth and Duplex Mode}

Frequency division duplexing was used for all simulations in chapter 4. The LTE downlink system bandwidth was then configured to be 10 or $20 \mathrm{MHz}$ depending on the study and employed the EPA or SCME channel models previously discussed. No model was used for the uplink since it was considered ideal in the simulations and only used for CSI reporting.

The system bandwidth determines the number of PRBs allocated to the base station, across the frequency domain, for downlink transmissions. A $10 \mathrm{MHz}$ downlink system 
Table 3.1: Base Simulation Parameters for LTE Downlink Throughput Comparisons

\begin{tabular}{|c|c|c|}
\hline Category & Parameter & Value \\
\hline \multirow{5}{*}{ System } & Bandwidth & 10 or $20 \mathrm{MHz}$ (50 or $100 \mathrm{PRBs}$ ) \\
\hline & Frequency & $2.6 \mathrm{GHz}$ \\
\hline & Duplex mode & FDD \\
\hline & Sub-carrier spacing & $15 \mathrm{kHz}$ \\
\hline & Cyclic prefix & Normal CP $(4.7 \mu s e c)$ \\
\hline \multirow{3}{*}{ Transmission } & Mode & TM2, TM3, TM4 \\
\hline & Antenna ports & 2 or 4 RS ports \\
\hline & RS power boosting & $3 \mathrm{~dB}$ or $6 \mathrm{~dB}$ \\
\hline Simulation & Duration & 15,000 sub-frames \\
\hline \multirow{2}{*}{ BS antennas (SCME) } & Configurations & 2 or 4 elements (Fig 4.2) \\
\hline & Spacing & $0.7 \lambda, 10 \lambda$ meters \\
\hline BS Antennas (EPA) & Correlation & Low, high correlation matrix \\
\hline \multirow{6}{*}{ Channel } & Channel model & SCME urban macro or EPA \\
\hline & Angular spreading & Low (SCME only) \\
\hline & \multirow{2}{*}{ UE velocity } & 3,30 and $120 \mathrm{~km} / \mathrm{hr}$ or \\
\hline & & $2.1,20.8$ and $124.6 \mathrm{~km} / \mathrm{hr}$ \\
\hline & \multirow{2}{*}{ SNR } & Swept ( -10 to $34 \mathrm{~dB})$ or \\
\hline & & Fixed $(15 \mathrm{~dB})$ \\
\hline \multirow{3}{*}{ Resources/Scheduling } & UE Resources & $8 \mathrm{PRB}$ or all PRB \\
\hline & FSS & Active if reporting sub-band CQI \\
\hline & OLLA & On or off \\
\hline \multirow{3}{*}{ Receiver Algorithms } & Channel estimation & MMSE \\
\hline & Synchronization & Ideal or practical (section 4.1.5) \\
\hline & Receiver front-end & Ideal or practical \\
\hline \multirow{4}{*}{ Reporting } & CQI reporting interval & $1,5,10$ or $20 \mathrm{msec}$ \\
\hline & CQI processing delay & $8 \mathrm{msec}$ or $2 \mathrm{msec}$ \\
\hline & CQI group size & $8 \mathrm{PRB}$ or wideband \\
\hline & PMI group size & $8 \mathrm{PRB}$ or wideband \\
\hline
\end{tabular}


bandwidth corresponds to $50 \mathrm{PRBs}$ in the frequency domain while a $20 \mathrm{MHz}$ bandwidth corresponds to 100 PRBs. The system bandwidth used in each simulation is given in table 3.2 .

Table 3.2: System Bandwidth used in Each Study

\begin{tabular}{cclc}
\hline Study & Section & \multicolumn{1}{c}{ Study Description } & System Bandwidth \\
\hline \hline $1 \mathrm{a}$ & 4.1 .5 & Comparison of the fall-back mode vs CLSM & 100 PRBs \\
$1 \mathrm{~b}$ & 4.1 .6 & Evaluation of various antenna configurations. & 50 PRBs \\
$2 \mathrm{a}$ & 4.2 .2 & Effect of generated phase noise on the PDSCH. & 100 PRBs \\
$2 \mathrm{~b}$ & 4.2 .3 & Effect of sampled phase noise on the PDSCH. & 100 PRBs \\
$3 \mathrm{a}$ & 4.3 .2 & Effect of UE velocity on the PDSCH. & 50 PRBs \\
$3 \mathrm{~b}$ & 4.3 .3 & PDSCH performance at high speed train velocity. & 50 PRBs \\
\hline
\end{tabular}

The number of PRBs in the frequency domain allocated to the UE may be less than the full system bandwidth. The UE resource allocation is discussed in section 3.4.6.

\subsubsection{Transmission Parameters}

\section{Transmission Mode}

The transmission modes used in each study were determined by the goal of each experiment discussed in chapter 4. Table 3.3 lists the transmission modes used in each study.

\section{Antenna Port}

Reference signals are transmit by the base station to permit the UE to estimate the channel for frequency domain equalization and analyze the channel to report the link adaptation parameters (CQI, RI and PMI if applicable). The number of antenna ports transmit determine the number of different channels (rank) that can be differentiated and estimated between the base station and UE antennas. The number of data streams in the channel is limited to the number of different cell-specific reference signals patterns transmit, also known as antenna ports, since the UE can only estimate and identify signals transmit using unique reference signals patterns. Cell-specific reference signals and antenna ports are discussed 
Table 3.3: Transmission Modes used in Each Study

\begin{tabular}{cclc}
\hline Study & Section & \multicolumn{1}{c}{ Study Description } & $\begin{array}{c}\text { Transmission } \\
\text { Mode }\end{array}$ \\
\hline \hline $1 \mathrm{a}$ & 4.1 .5 & Comparison of the fall-back mode to CLSM. & TM2, TM4 rank-1 \\
\hline $\mathrm{b}$ & 4.1 .6 & Evaluation of various antenna configurations. & TM3, TM4 \\
\hline $\mathrm{a}$ & 4.2 .2 & Effect of generated phase noise on the PDSCH. & TM2, TM4 rank-1 \\
$2 \mathrm{~b}$ & 4.2 .3 & Effect of sampled phase noise on the PDSCH. & TM3, TM4 \\
$3 \mathrm{a}$ & 4.3 .2 & Effect of UE velocity on the PDSCH. & TM3, TM4 \\
$3 \mathrm{~b}$ & 4.3 .3 & PDSCH performance at high speed train velocity. & TM3, TM4 \\
\hline
\end{tabular}

in section 2.4.4 and the three CRS patterns used in this thesis are shown in figures 2.29, 2.30 and 2.31. Finally, the antenna port setting used in the simulations conducted for this thesis are listed in table 3.4.

Table 3.4: Antenna Ports used in Each Study

\begin{tabular}{cclc}
\hline Study & Section & \multicolumn{1}{c}{ Study Description } & Antenna Ports \\
\hline \hline $1 \mathrm{a}$ & 4.1 .5 & Fall-back mode vs CLSM. & 4-Tx 4-Ports \\
$1 \mathrm{~b}$ & 4.1 .6 & Antenna configuration evaluation. & 2-Tx 2-Port, 4-Tx 2-Port, 4-Tx 4-Port \\
$2 \mathrm{a}$ & 4.2 .2 & Effect of generated phase noise. & 4-Tx 4-Port \\
$2 \mathrm{~b}$ & 4.2 .3 & Effect of sampled phase noise. & 4-Tx 4-Port \\
$3 \mathrm{a}$ & 4.3 .2 & Effect of UE velocity. & 2-Tx 2-Port, 4-Tx 2-Port, 4-Tx 4-Port \\
$3 \mathrm{~b}$ & 4.3 .3 & High speed train performance. & 4-Tx 4-Ports \\
\hline
\end{tabular}

\section{Reference Signal (RS) Power Boosting}

Reference signal power boosting is used to increase the accuracy of the channel estimate at the UE for improved decoding. Reference signal sub-carrier power can be increased if the maximum power transmit per antenna is observed by "borrowing" the power from other data or reference signal resource elements in the time-frequency resource map grid. 
Borrowing power for reference signal power boosting may be accomplished without reducing data RE power by borrowing power from unused sub-carriers on the same antenna port. For instance, when four antenna ports are used, the sub-carriers corresponding to the reference signals of ports $P_{1}, P_{2}$ and $P_{3}$ are muted on antenna port $P_{0}$ and no data is transmit in those sub-carriers to avoid causing interference. Since no power is transmit on the muted sub-carriers corresponding to the reference signals for antenna ports $P_{1}, P_{2}$ and $P_{3}$ on antenna port $P_{0}$ the unused power can be used to boost other resource elements on antenna port $P_{0}$, including boosting antenna port $P_{0}$ reference signals.

RS power boosting is beneficial in poor channel conditions because the boosted RS sub-carriers increase the SNR of the reference signals at the receiver and can potentially improve the channel estimate at the receiver, potentially leading to improved decoding. The simulations performed for this thesis use the RS power boosting settings shown in table 3.5.

Table 3.5: Reference Signal Power Boosting used in Each Study

\begin{tabular}{cclc}
\hline Study & Section & \multicolumn{1}{c}{ Study Description } & RS Power Boosting \\
\hline \hline $1 \mathrm{a}$ & 4.1 .5 & Fall-back mode vs CLSM. & $3 \mathrm{~dB}$ \\
$1 \mathrm{~b}$ & 4.1 .6 & Antenna configuration evaluation. & $3 \mathrm{~dB}$ \\
$2 \mathrm{a}$ & 4.2 .2 & Effect of generated phase noise. & $6 \mathrm{~dB}$ \\
$2 \mathrm{~b}$ & 4.2 .3 & Effect of sampled phase noise. & $6 \mathrm{~dB}$ \\
$3 \mathrm{a}$ & 4.3 .2 & Effect of UE velocity. & $3 \mathrm{~dB}$ \\
$3 \mathrm{~b}$ & 4.3 .3 & High speed train performance. & $3 \mathrm{~dB}$ \\
\hline
\end{tabular}

\subsubsection{Simulation Duration}

Time-averaging was used to increase the accuracy of the throughput results for the LTE downlink simulations. 15,000 sub-frames were simulated per SNR or velocity point, corresponding 210,000 OFDM symbols, to ensure convergence of the throughput results.

\subsubsection{Antenna Simulation Parameters}

The UE antenna configuration is fixed to two cross-polarized antennas for all the simulations discussed in chapter 4 , as depicted in figure 4.3a in section 4.1.2. 
The available base station antenna configurations for the SCME channel model are depicted in figure 4.2 in section 4.1.1. The antenna configurations are varied to determine the performance of various configurations. Two and four elements are evaluated along with two and four antenna ports and the antenna correlations discussed in section 3.4.4.

In the antenna configuration simulation a number of antenna configurations are compared, however, in later simulations the subset of antennas per simulation is reduced to keep the simulations manageable. The antenna configurations with the best results observed for the SCME drop and EPA channel model are re-used, while other configurations, such as the correlated co-polarized antennas are dropped entirely from the figures in later simulations.

\section{Base Station Antenna Correlation}

In the simulations presented in this thesis, a low and high antenna correlation setting was chosen to observe the performance of the LTE downlink when widely spaced antennas are employed versus closely spaced antennas. The correlation settings were chosen to evaluate the transmission schemes using the two possible extremes of antenna correlation. Widely spaced antennas are expected to improve the performance of transmission schemes like TM2 that benefit from diversity, while the closely spaced antennas are required for beamforming, used in TM3, TM4 and TM6.

\section{Setting Base Station Antenna Correlation for the EPA Channel Model}

When using the EPA channel model, the user is required to select a MIMO correlation matrix to set the level of correlation between the antenna elements because the simulator does not use, and is unaware of, the geometry and antenna physical description. The available MIMO correlation matrices for the conformance testing channel models are found in appendix B.2.3 of [6] and appendix B.5.1 of [4]. In this thesis, the low and high channel correlation matrices found in the appendices of [6] and [4] are used to compare the performance of various transmission schemes and antenna configurations using low and high antenna correlation.

The low and high correlation matrices are also used to compare EPA throughput curves to SCME throughput curves obtained with antenna element spacings of $10 \lambda$ and $0.7 \lambda$. 


\section{Setting Base Station Antenna Correlation for the SCME}

In the SCME simulations, the antenna element correlation is dependent on the geometry provided by the user. The base station antenna elements and the UE antenna elements are positioned, oriented and tilted relative to the base station and UE location and orientation. The antenna array origins are centered at the origin of the base station and the UE at heights of $h_{b s}=30$ meters and $h_{m s}=1$ meters, respectively. To position the antenna elements, the element coordinates are given relative to the antenna arrays, themselves.

The distance separating the antenna elements, after the complete array has been defined, determines the correlation between the antenna elements in the SCME. Two antenna spacings were chosen in order to be able to compare SCME throughput results to the EPA throughput curves with similar correlation. For the SCME, an element spacing of $10 \lambda$ was used to represent the low antenna correlation, while high correlation was achieved using a spacing of $0.7 \lambda$.

Figure 4.2 and figure 4.1 show the antenna configurations and the spacings used to define the base station antenna array in the SCME simulations in chapter 4.

\subsubsection{Channel Simulation Parameters}

\section{Channel Models}

The channel models used in chapter 4 are listed in table 3.6. Both the EPA channel model and the spatial channel model are used, for comparison purposes. The spatial channel model was described in section 2.3.7 and is the preferred channel model for spatial multiplexing transmission modes due to the improved spatial characteristics modeling. 
Table 3.6: Channel Models used in Each Study

\begin{tabular}{cclc}
\hline Study & Section & \multicolumn{1}{c}{ Study Description } & Channel Model \\
\hline \hline 1a & 4.1 .5 & Comparison of the fall-back mode to CLSM. & EPA, SCME \\
$1 \mathrm{~b}$ & 4.1 .6 & Evaluation of various antenna configurations. & SCME \\
$2 \mathrm{a}$ & 4.2 .2 & Effect of generated phase noise on the PDSCH. & EPA \\
$2 \mathrm{~b}$ & 4.2 .3 & Effect of sampled phase noise on the PDSCH. & SCME \\
$3 \mathrm{a}$ & 4.3 .2 & Effect of UE velocity on the PDSCH. & SCME \\
$3 \mathrm{~b}$ & 4.3 .3 & PDSCH performance at high speed train velocity. & SCME \\
\hline
\end{tabular}

\section{Angular Spreading (AS) at the Base Station for the SCME}

The channel angular spreading at the base station is an environment parameter defined in table 5.1 of [3]. For the Urban Macro channel scenario, the user has the option of setting the mean angular spreading at the BS to $8^{\circ}$ or $15^{\circ}$ corresponding to low and high angular spreading, respectively. The mean angular spreading user parameter sets the standard deviation of the distribution used to draw the angles of departure of the paths departing the base station and the mean angle spread selected by the user determines the multi-path richness of the channel.

All $M=20$ sub-paths in the SCME have the same path power $P_{n} / M$, however, the randomly drawn angles of departure of the paths combined with the sub-path fixed angles of departure defined in table 5.2 of [3] cause the sub-paths to experience different antenna gains. A wider mean angle spread is therefore associated with a lower correlation between the paths in the channel.

A low angular spreading setting was used in the results in this thesis to restrict the number of simulations and focus on the important parameters within each study.

\section{Doppler and UE Velocity}

The channel Doppler values used in the simulations in this thesis are roughly based on the standard maximum Doppler frequencies given in appendix B.2 of technical specification 36.104 [4].

Two sets of UE velocities are used in this thesis. The first set $\{2.1,20.8$ and 124.6\} $\mathrm{km} / \mathrm{hr}$ was used to compare results to prior work and are similar at low and high velocity. 
The second set of UE velocities $\{3,30$ and 120$\} \mathrm{km} / \mathrm{hr}$ are similar to the standard maximum Doppler frequencies given in appendix B.2 of technical specification 36.104 [4] but are rounded for simplicity and often used in papers and other publications.

In table 3.7, the UE velocities used in this thesis are compared to the exact UE velocities calculated using the standard maximum Doppler frequencies from appendix B.2 of [4] and the carrier frequency. Where there are differences between the UE velocities used in chapter 4 of this thesis and the exact UE velocity, the corresponding maximum Doppler frequency $f_{m}$ is given for comparison. The author is cautious not to compare the results using differing UE velocities unless the difference is small enough to not significantly affect the validity of the comparison.

Table 3.7: UE Velocities used in this Thesis

\begin{tabular}{cc|cc}
\hline $\begin{array}{c}\text { max. Doppler } \\
\text { Frequency }\end{array}$ & $\begin{array}{c}\text { Actual UE Velocity } \\
\text { converted from Doppler Values }\end{array}$ & $\begin{array}{c}\text { UE Velocities used } \\
\text { In Some Simulations }\end{array}$ & $\begin{array}{c}\text { Standard UE } \\
\text { Velocity }\end{array}$ \\
\hline \hline $3 \mathrm{~Hz}$ & $1.2 \mathrm{~km} / \mathrm{hr}$ & $2.1 \mathrm{~km} / \mathrm{hr}(\sim 5 \mathrm{~Hz})$ & $3 \mathrm{~km} / \mathrm{hr}(\sim 7 \mathrm{~Hz})$ \\
$70 \mathrm{~Hz}$ & $28.9 \mathrm{~km} / \mathrm{hr}$ & $20.8 \mathrm{~km} / \mathrm{hr}(\sim 50 \mathrm{~Hz})$ & $30 \mathrm{~km} / \mathrm{hr}(\sim 72 \mathrm{~Hz})$ \\
$300 \mathrm{~Hz}$ & $124.6 \mathrm{~km} / \mathrm{hr}$ & $124.6 \mathrm{~km} / \mathrm{hr}$ & $120 \mathrm{~km} / \mathrm{hr}(\sim 289 \mathrm{~Hz})$ \\
\hline
\end{tabular}

Equation 3.3 is used to convert the UE velocities used in chapter 4 to a corresponding maximum Doppler Frequency $f_{m}$ in table 3.7. The carrier frequency $f_{c}$ used for the conversion is $2.6 \mathrm{GHz}$.

$$
v=\frac{f_{m} \cdot c}{f_{c}}, \text { where } c \approx 3 \times 10^{8}
$$

\section{Signal-to-Noise Ratio (SNR)}

In the majority of the simulations an SNR range was used to obtain and observe the performance of the LTE downlink from -10 to 30 or $34 \mathrm{~dB}$. The SNR was fixed to $15 \mathrm{~dB}$ in the study in section 4.3.2 in order to plot the throughput of the system versus the UE velocity. 


\subsubsection{Resource Allocation and Scheduling}

\section{UE Resource Allocation}

The UE resource allocation determines the amount of spectral resources allocated to the UE for the transmission of data to the UE. The amount of spectral resources are measured in PRBs in the frequency domain and two settings were used in the simulations in this thesis.

UE resource allocation settings used in this thesis:

- 8 PRBs, to represent a typical UE allocation of $1.44 \mathrm{MHz}$ or 96 sub-carriers.

- Entire bandwidth (All available PRBs), to measure the performance of the complete LTE downlink at the physical layer.

Table 3.8 is a list of the spectral resource allocation settings used in the simulations conducted for this thesis.

Table 3.8: Spectral Resources Allocated to the UE in Each Study

\begin{tabular}{cclc}
\hline Study & Section & \multicolumn{1}{c}{ Study Description } & UE Resource Allocation \\
\hline \hline $1 \mathrm{a}$ & 4.1 .5 & Fall-back mode vs CLSM. & $8 \mathrm{PRBs}$ \\
$1 \mathrm{~b}$ & 4.1 .6 & Antenna configuration evaluation. & $50 \mathrm{PRBs}$ \\
$2 \mathrm{a}$ & 4.2 .2 & Effect of generated phase noise. & $8 \mathrm{PRBs}$ \\
$2 \mathrm{~b}$ & 4.2 .3 & Effect of sampled phase noise. & $8 \mathrm{PRBs}$ \\
$3 \mathrm{a}$ & 4.3 .2 & Effect of UE velocity. & $8 \mathrm{PRBs}$ \\
$3 \mathrm{~b}$ & 4.3 .3 & High speed train performance. & $50 \mathrm{PRBs}$ \\
\hline
\end{tabular}

\section{Frequency Selective Scheduling (FSS)}

LTE's frequency selective scheduling feature described in section 2.4.6 is an important feature made possible by OFDM technology since it helps overcome the channel effect known as frequency selective fading discussed in section 2.1.3. Frequency selective scheduling is enabled when sub-band reporting is enabled and the CQI group size is set to 8 PRB, permitting the scheduler to schedule the UE downlink transmissions in best possible portion of the wireless channel in the frequency domain. 


\section{Outer-Loop Link Adaptation (OLLA)}

Outer-loop link adaptation is used in all but the UE velocity and high speed train simulations, in section 4.3.2 and section 4.3.3, to adapt the MCS used for transmission based on the HARQ ACK and NACK responses sent by the UE. While OLLA typically provides throughput gain, it was not used in the UE velocity and high speed train simulations, as shown in table 3.9, in order to isolate the effect of the rapidly changing channel and the link adaptation loop on the decoding of the PDSCH.

Table 3.9: Outer-loop Link Adaptation Settings used in Each Study

\begin{tabular}{cclc}
\hline Study & Section & \multicolumn{1}{c}{ Study Description } & OLLA \\
\hline \hline $1 \mathrm{a}$ & 4.1 .5 & Fall-back mode vs CLSM. & On \\
$\mathrm{1b}$ & 4.1 .6 & Antenna configuration evaluation. & On \\
$2 \mathrm{a}$ & 4.2 .2 & Effect of generated phase noise. & On \\
$2 \mathrm{~b}$ & 4.2 .3 & Effect of sampled phase noise. & On \\
$3 \mathrm{a}$ & 4.3 .2 & Effect of UE velocity. & Off \\
$3 \mathrm{~b}$ & 4.3 .3 & High speed train performance. & Off \\
\hline
\end{tabular}

\subsubsection{Receiver Algorithms}

\section{Channel Estimation and Synchronization}

Channel estimation and synchronization were introduced in section 2.4.5 and 2.4.3. MMSE channel estimation and practical synchronization algorithms were used to model a practical UE in all simulations in chapter 4, except for in the UE velocity simulations in section 4.3.2. In section 4.3.2 ideal channel estimation and synchronization were used to highlight the effect of the link adaptation control loop processing delay and reporting interval without additional degradation caused by channel estimation error.

When practical synchronization is used, the known synchronization signals are used to determine the timing offset. On the other hand, ideal synchronization is determined by finding the propagation delay of the strongest MPCs. 


\section{Receiver Front-End}

The simulator is configured to model the front-end of a practical receiver. Filtered noise with standard deviation of $1.5^{\circ} \mathrm{RMS}$ and $10 \mathrm{kHz}$ bandwidth is added to the received signal to model the phase noise of a practical local oscillator performing down-conversion at the receiver. In addition, an I-Q imbalance is introduced with a magnitude of $0.5 \mathrm{~dB}$ and phase $1.5^{\circ}$.

\subsubsection{Channel State Information (CSI) Reporting for Link Adapta- tion (LA)}

Channel state information for link adaption is reported to the eNodeB using either the PUCCH or PUSCH uplink channels in LTE. In the link level simulator a simple lossless uplink channel was used. The link adaptation reporting interval and the processing delay caused by the CQI selection algorithm calculation, uplink transmission and processing at the eNodeB are used to more realistically model the link adaptation control loop that adjusts the MCS, RI and PMI to track the changing wireless channel.

\section{CQI Reporting Interval and Processing Delay}

The processing delays and reporting intervals depicted in the example in figure 4.27 are used to model and evaluate the effects of practical link adaptation algorithms in the LTE radio access network. The UE is configured to send channel state information reports to the eNodeB at intervals of 1 or $5 \mathrm{msec}$ in the majority of the simulations along with a practical processing delay of $8 \mathrm{msec}$, as shown in table 3.10. The frequent reporting intervals of 1 or $5 \mathrm{msec}$ are needed to track the channel even at relatively low velocities.

In the UE velocity simulations in section 4.3.2, the UE velocity is swept for different reporting intervals (1, 10 and $20 \mathrm{msec}$ ) and processing delays (2 and $8 \mathrm{msec}$ ) in order to determine the effect of the lsink adaptation parameters on the throughput of the LTE downlink.

\section{CQI and PMI Group Size}

The CQI group size and the PMI size are shown in table 3.11 and are selected to permit FSS when the UE is allocated $8 \mathrm{PRB}$ in the frequency domain and the CQI group size is 
Table 3.10: Link Adaptation Settings used in Each Study

\begin{tabular}{cclcc}
\hline Study & Section & \multicolumn{1}{c}{$\begin{array}{c}\text { Study } \\
\text { Description }\end{array}$} & $\begin{array}{c}\text { Processing } \\
\text { Delay }\end{array}$ & $\begin{array}{c}\text { Reporting } \\
\text { Interval }\end{array}$ \\
\hline \hline 1a & 4.1 .5 & Fall-back mode vs CLSM. & $8 \mathrm{msec}$ & $5 \mathrm{msec}$ \\
$1 \mathrm{~b}$ & 4.1 .6 & Antenna configuration evaluation. & $8 \mathrm{msec}$ & $1 \mathrm{msec}$ \\
$2 \mathrm{a}$ & 4.2 .2 & Effect of generated phase noise. & $8 \mathrm{msec}$ & $5 \mathrm{msec}$ \\
$2 \mathrm{~b}$ & 4.2 .3 & Effect of sampled phase noise. & $8 \mathrm{msec}$ & $5 \mathrm{msec}$ \\
$3 \mathrm{a}$ & 4.3 .2 & Effect of UE velocity. & 2 or $8 \mathrm{msec}$ & $1,10 \mathrm{or} 20 \mathrm{msec}$ \\
$3 \mathrm{~b}$ & 4.3 .3 & High speed train performance. & $8 \mathrm{msec}$ & $1 \mathrm{msec}$ \\
\hline
\end{tabular}

8 PRB. The wideband setting for either the CQI or PMI indicates that the UE reports are applicable to the entire allocated bandwidth.

Table 3.11: Sub-band CQI/PMI Group Size Settings used in Each Study

\begin{tabular}{cclcc}
\hline Study & Section & \multicolumn{1}{c}{$\begin{array}{c}\text { Study } \\
\text { Description }\end{array}$} & $\begin{array}{c}\text { CQI Group } \\
\text { Size }\end{array}$ & $\begin{array}{c}\text { PMI Group } \\
\text { Size }\end{array}$ \\
\hline \hline $1 \mathrm{a}$ & 4.1 .5 & Fall-back mode vs CLSM. & 8 PRBs & 8 PRBs \\
$1 \mathrm{~b}$ & 4.1 .6 & Antenna configuration evaluation. & 8 PRBs & Wideband PRBs \\
$2 \mathrm{a}$ & 4.2 .2 & Effect of generated phase noise. & 8 PRBs & 8 PRBs \\
$2 \mathrm{~b}$ & 4.2 .3 & Effect of sampled phase noise. & 8 PRBs & 8 PRBs \\
$3 \mathrm{a}$ & 4.3 .2 & Effect of UE velocity. & 8 PRBs & Wideband PRBs \\
$3 \mathrm{~b}$ & 4.3 .3 & High speed train performance. & Wideband PRBs & Wideband PRBs \\
\hline
\end{tabular}

\section{Hybrid-ARQ (HARQ)}

Hybrid-ARQ was configured to operate 8 processes with the capability of 3 retransmissions attempts each after the first transmission. 


\subsection{Performance Benchmarks}

\subsubsection{Throughput Performance Benchmarking}

The simulation results for transmit diversity and the spatial multiplexing schemes used in chapter 4 were compared to LTE SFBC and spatial multiplexing results from prior work by the authors of [28], [32], [33], [34] and [35] to validate the throughput performance.

The spatial channel model was used to obtain a number of simulation results in chapter 4 along with the extended pedestrian model. While the EPA model is representative of an average pedestrian scenario in a low delay spread environment, the SCME is specific to the channel generated by the high level parameters, distributions set by the environment selection, chosen UE and BS location and the random number generator seed(s). For the SCME, fixing the geometry and distributions and channel seed result in a specific drop at each simulation. More than one drop needs to be simulated at different UE locations to get a distribution of the throughput achievable across the cell. While the SCME results are specific to the geometry, distributions and channel seed, reproducing the same channel is needed in this thesis to make fair comparisons between transmission modes, antenna configurations and various other inputs and settings under the same channel conditions.

\subsubsection{UE Velocity Benchmarking}

Results achieved for the throughput versus UE velocity simulations and link adaptation parameters were compared to the normalized throughput curves in [36]. The resulting degradation observed in [36] is similar to that observed in this thesis. For practical link adaptation settings ${ }^{1}$ the throughput loss observed in this thesis was between $29 \%$ and $34 \%$ of the throughput achieved at low velocity versus $30 \mathrm{~km} / \mathrm{hr}$. Similarly, as velocity increased in the figures in [36] the transmission mode that depends the most on the accuracy of the channel state information reported to the eNodeB showed the most degradation. Openloop spatial multiplexing is more robust in varying channel conditions then closed-loop spatial multiplexing as acknowledged by the authors of [36] and mirrored in the results in section 4.3.2. The most significant decrease in throughput performance is below 15 $\mathrm{km} / \mathrm{hr}$ in both [36] and the results in this thesis followed by a plateau in the reduction of the throughput above $15 \mathrm{~km} / \mathrm{hr}$. The results from the paper and this thesis offer similar conclusions including the suggestion that the performance of spatial multiplexing depends

\footnotetext{
${ }^{1}$ For the practical link adaptation settings a $10 \mathrm{msec}$ reporting interval and $8 \mathrm{msec}$ total processing delay was assumed.
} 
on the practical link adaptation reporting interval and delays, UE velocity and that due to each spatial multiplexing mode strengths and weaknesses, proper choice of the spatial multiplexing mode with respect to UE velocity is important to maximize the overall system performance. 


\section{Chapter 4}

\section{Simulation Results}

\subsection{Antenna Configuration Performance}

The evaluation of antenna performance is a good place to start discussing the simulation results in this thesis since the focus of this thesis is the performance of the 4 transmitter antenna package. In addition, the simulation topics that follow the antenna performance results will build on the antenna performance results by adding noise and feedback impairments.

In wireless systems, the antenna design has a large impact on the performance of an OFDM system. Since the ITU-R and IMT-Advanced have set ambitious goals for the next generation wireless networks, current and near-future wireless network efficiencies require multi-antenna systems capable of advanced spatial multiplexing and beamforming transmissions to achieve these goals.

Multiple transmit and receive antennas have been a key technology that has permitted increases in the spectral efficiency of commercial wireless systems, such as 802.11, digital audio broadcast (DAB) and LTE to name a couple. The number of antennas that can be used in an LTE system depends on factors like the amount of space available, the cost to the operator, the physical appearance and the regulations of the state governing the mounting of base station antennas in urban or sub-urban areas. The impact of the antenna configuration on the LTE downlink throughput needs to be assessed in order to be able to recommend viable options to the operator to keep cost low while offering adequate coverage and service speeds. Armed with the knowledge presented in the next section, system designers will be able to advise operators on the best antennas for particular scenarios and to help make better use of the available spectrum resources. 


\subsubsection{Base Station Antenna Configurations}

This section introduces the 7 possible antenna configurations based on the uniform linear array (ULA) antenna. The BS antenna configurations cover a number of different cases including various polarization, antenna correlation and the number of antennas and antenna ports. The number of antennas simulated will be 2 and 4 antennas using 2 different antenna spacings to achieve high and low antenna correlation. 2 antenna base station simulation results will be presented next to the 4 antenna base station results to look at the potential increase in throughput that can be achieved by the more complex 4 antenna system.

\section{Number of Antennas and Antenna Ports at the Base Station}

Antenna ports were first discussed in section 2.4.4 and graphically depicted in figure 2.29 for 2-Tx 2-port, figure 2.30 for 4-Tx 2-port and figure 2.31 for 4-Tx 4-port. The antenna port reference signals, shown in section 2.4 .4 , are used by the system to perform channel estimation and are always broadcast across the frequency domain in the allocated LTE downlink system bandwidth. The UE performs channel estimation based on the reference signal pattern received from each BS antenna port. The reference signal patterns can be unique as in the case of 2-Tx 2-port and 4-Tx 4-port spatial multiplexing schemes where the antenna port RS patterns used are the $\operatorname{RS}$ patterns for ports $\{0,1\}$ and ports $\{0,1,2,3\}$ respectively.

The number of antenna ports $P$ determines the number of possible layers $v$ and codewords $q$ that can be used during transmission since the condition $q \leq v \leq P$ must be met. Therefore, from a throughput perspective, a lower number of antennas and antenna ports may limits the performance of the system. For instance, in a 4-Tx 4-port system the receiver will be able to identify and estimate 8 channels (4 ports $x 2$ receivers), while in a 4-Tx 2port system only 4 channels ( 2 ports $\mathrm{x} 2$ receivers) can be estimated. On the other hand, each port adds reference signal overhead to the LTE transmissions therefore the simulations will help determine what combination of transmit antennas and ports will be optimal.

\section{Base Station Element Correlation in SCME Simulations}

The correlation between the BS antennas is changed by varying the distance between the elements in the SCME simulations. Two spacings were chosen to represent the high correlation and low correlation antenna elements in the SCME simulations. A spacing of $10 \lambda$ 
was chosen for the low correlation case based on an existing customer antenna specification, and the $0.7 \lambda$ spacing was selected to represent the high correlation case which is near the optimal beamforming spacing.

\section{Base Station Element Correlation in Extended-ITU Simulations}

In some cases, the extended-International Telecommunication Union (ITU) fixed tap delay line channel model named EPA has been used for simulation purposes. The extended-ITU models are referred to as fixed tapped delay line (TDL) models since the tap locations in time, or tap delays $\tau_{n}$, do not change and the average tap powers are also fixed and as described in [6]. The models use correlation matrices to model the correlation between antenna elements instead of angles, such as the AoD and AoA and channel angular spreading setting that are used in the SCM, SCME and WINNER channel models.

The antenna correlation for the extended-ITU models (EPA, EVA and ETU) are predefined, as shown in appendix D.1 of this thesis and in appendix B.2.3.1 of the 3GPP technical specification (TS) 36.101 [6]. The user selects the desired level of correlation for the fixed TDL simulation from the set \{low, medium, high\} which in turn defines the values for $\alpha$, the correlation between the BS antennas and $\beta$, the correlation between UE antennas. The selected correlation option sets the BS antenna correlation and then the same matrix is applied to each multi-path component (MPC) of the fixed TDL channel model.

In the case where the EPA model is used in this thesis, the correlation setting is either "high" or "low", and the channel model uses the corresponding 3GPP supplied correlation matrices listed ${ }^{1}$ in [6] during simulation.

\section{Base Station Antenna Configurations}

The base station antenna configurations that will be evaluated are shown in figures 4.1 and figure 4.2.

\footnotetext{
${ }^{1}$ Correlation matrices for the $2 \times 2 \mathrm{MIMO}$ channel are given as references in appendix D.1, the reader is referred to [6] for additional correlation matrices and information on this subject.
} 


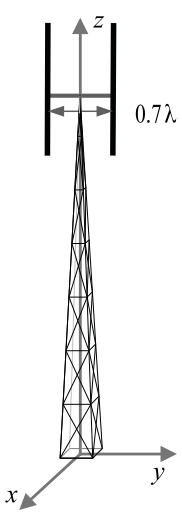

(a) 2 Elements $(0.7 \lambda$ Spacing $)$
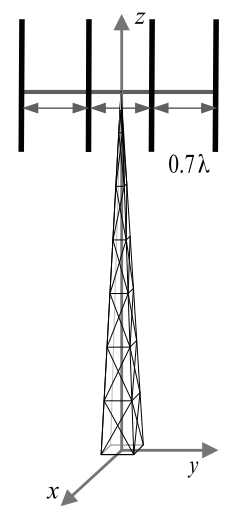

(c) 4 Elements $(0.7 \lambda$ Spacing $)$

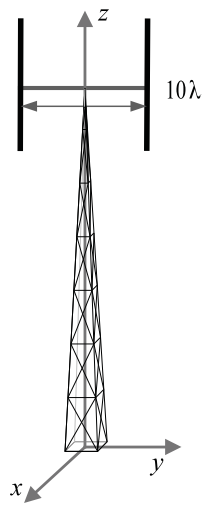

(b) 2 Elements (10ג Spacing)

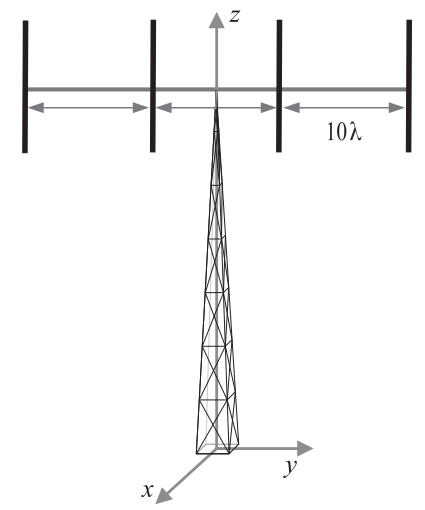

(d) 4 Elements (10 $\lambda$ Spacing)

Figure 4.1: Co-polarized BS Antenna Element Configurations

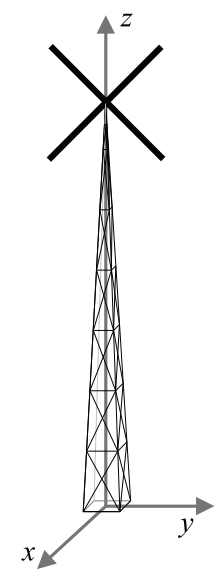

(a) 2 Elements

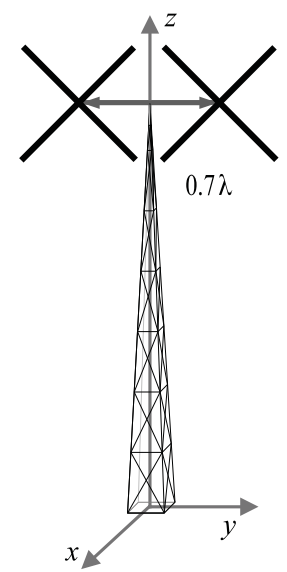

(b) 4 Elements $(0.7 \lambda$ Spacing)

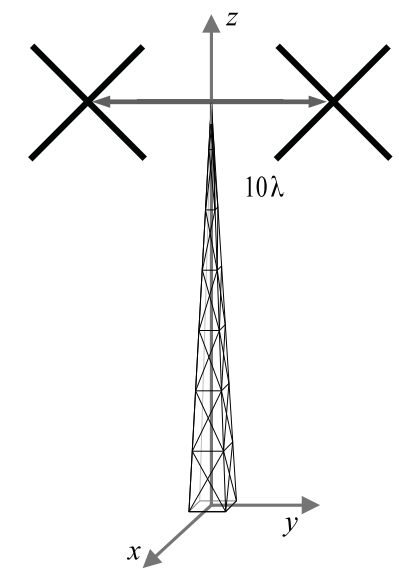

(c) 4 Elements (10 $\lambda$ Spacing)

Figure 4.2: Cross-Polarized BS Antenna Element Configurations 


\subsubsection{Mobile Station Antenna Configuration}

The increased use of data and spatial multiplexing technology in wireless communications requires better modeling of the mobile devices receiving antenna configuration, its location in the cell and the angle of the antenna(s), also known as tilt. The handset position has changed to a data dominated position in recent years with the increased use of text messaging services, email and Internet browsing, where the latter requires high speed data connections for video and rich content. Tablets and laptops emphasize the shift further since the primary use of mobile handheld tablets is accessing the Internet and rich content services with no traditional "talk" position.

Table 4.3 depicts the possible antenna configurations for the mobile phone and laptop or tablet devices. Due to space constraints in smaller devices, such as traditional phones or smart phones, separating the antennas by large distances to ensure uncorrelated received signals is difficult and is acknowledged in section 5.5.1 of [3]. The spacings required to achieve low correlation for 2 widely used LTE carrier frequencies are given in table 4.1. Based on the required antenna spacings in table 4.1 it is difficult to integrate co-polarized antennas since the antennas are essentially co-located and would not provide substantial diversity gain. The solution is to use polarization diversity as mentioned in section 5.5.1 of [3]. By using cross-polarized antenna elements a significant level of isolation between the cross-polarized elements is achieved and the elements can be assumed to be uncorrelated. The level of isolation between antenna elements can be set in the SCME simulations using the cross-polarization discrimination ratio (XPD).

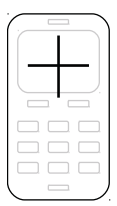

(a) 2 Elements MS

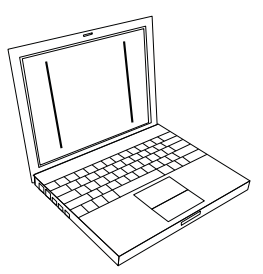

(b) 2 Element Laptop

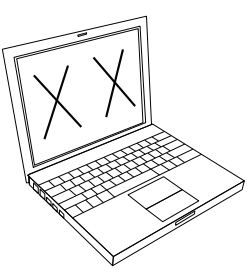

(c) 4 Elements Laptop

Figure 4.3: MS Antenna Configurations

In addition, the benefits of using cross-polarized antennas in the mobile devices were shown in [37]. In [37], the cross-polarized elements achieve higher throughput than the co-polarized antennas. Figure 3 in [37] shows that the proportion of rank-2 transmissions increases by the use of cross-polarized elements, when compared to tests with co-polarized 
Table 4.1: Wavelengths and Antenna Element Spacing of Two Common LTE Frequency Bands

\begin{tabular}{cccc}
\hline RF Band & Wavelength & $\begin{array}{c}\text { Correlated } \\
\text { Elements }(0.7 \lambda)\end{array}$ & $\begin{array}{c}\text { Uncorrelated } \\
\text { Elements }(10 \lambda)\end{array}$ \\
\hline \hline $700 \mathrm{MHz}$ & $42.9 \mathrm{~cm}$ & $30.0 \mathrm{~cm}$ & $428.6 \mathrm{~cm}$ \\
$2600 \mathrm{MHz}$ & $11.54 \mathrm{~cm}$ & $8.1 \mathrm{~cm}$ & $115.4 \mathrm{~cm}$ \\
\hline
\end{tabular}

UE antennas. The high throughput regions of the cumulative distribution function (CDF) and the distribution of the transmission rank (1-4) in the stacked bar graph show that far more rank-2 and rank-3 transmissions are possible with cross-polarized antennas. Therefore, for all the simulations presented in this thesis the selected UE antenna configuration is the 2 cross-polarized elements.

\subsubsection{Summary of the Antenna Configurations}

Based on the number of antennas available at the base station and the fixed cross-polarized antennas at the UE, the following system configurations will be simulated. The short hand names in parentheses, in the list below, will be used to refer to each antenna configuration from this point forward.

The antenna configurations used in the simulations in this thesis are:

- 2 transmitters $\times 2$ receivers, 2 antenna ports (2-Tx 2-port)

- 4 transmitters x 2 receivers, 2 antenna ports (4-Tx 2-port)

- 4 transmitters $x 2$ receivers, 4 antenna ports (4-Tx 4-port)

A summary of all the possible options including polarization, correlation and number of elements and ports for the base station are given in table 4.2.

\subsubsection{Transmission Modes for Antenna Configuration Simulations}

In the antenna configurations simulations, 3 transmission modes will be simulated and compared, namely transmit diversity and open and closed-loop spatial multiplexing. The 
Table 4.2: BS and MS Antenna Configurations

\begin{tabular}{clc}
\hline \multicolumn{1}{c}{ Entity } & \multicolumn{1}{c}{ Parameter } & \multicolumn{1}{c}{ Configurations } \\
\hline \hline \multirow{3}{*}{ Base Station } & Number of antenna elements & 2,4 \\
& Polarizations & co-polarized, cross-polarized \\
& Number of antenna ports & $2,4^{\mathrm{a}}$ \\
& Antenna correlation & correlated $(0.7 \lambda)$, uncorrelated $(10 \lambda)$ \\
\hline \multirow{2}{*}{ Mobile Station } & Number of antenna elements & 2 \\
& Polarization & cross-polarized \\
\hline
\end{tabular}

a Four antenna ports are only possible when 4 antenna elements are used.

3 modes will be simulated to show the throughput performance of each mode using different antenna configurations. Transmission modes are defined in LTE due to their potential strengths in various environments. For instance, transmit diversity using SFBC or SFBCFSTD is known to offer good coverage but only a single codeword is used per transmission.

On the other hand, in LTE the spatial multiplexing schemes may attain a higher peak throughput if the channel conditions allow multiple codewords to be transmit. The reason is that increasing the number of antennas when using spatial multiplexing schemes in theory increases the performance of a wireless system by allowing finer resolution beamforming, increased diversity and up to $\min \left(N_{t}, N_{r}\right)$ codewords to be sent due a higher possible channel rank with increased transmitters $N_{t}$ or receivers $N_{r}$. While the available LTE transmission modes can be paired with most antenna configurations, the performance of the system needs to be understood in order to deploy the best mode possible in a particular wireless environment. Evaluating the transmission modes may also assist in the development of future self organizing networks (SONs) which could automatically measure the environment and change the LTE system settings or transmission modes without the need for operator input. 
Three modes are of interest in this study and a fourth, TM4 rank-1 is used for fair comparison with TM2. The modes of interest are:

- TM2: Transmit diversity (reference) (refer to section 2.5.2)

- TM3: Open-loop spatial multiplexing (refer to section 2.5.3)

- TM4: Closed-loop spatial multiplexing (refer to section 2.5.4)

- TM4 rank-1: CLSM limited to rank-1 transmissions (refer to section 2.5.4 and 2.5.5)

The first mode TM2 (transmit diversity) is a robust mode discussed in section 2.5.2. TM2 is used as a baseline to benchmark the more complex spatial multiplexing transmission modes TM3 and TM4, discussed in section 2.5.3 and section 2.5.4, respectively. Spatial multiplexing modes will also be evaluated. The spatial multiplexing modes take advantage of MIMO channels to increase the number of possible codewords up to 2 and transmission layers up to 4 . In addition, precoding of the layers allows for beamforming to occur. TM3 uses a fixed precoder with 2 BS antennas and CDD when using 4 BS antennas. TM4 uses the optimal precoder based on PMI feedback from the UE. The antenna configuration affects the performance of the 3 transmission modes in different ways. This section will investigate the throughput performance of the 3 modes with the antenna configurations previously summarized in section 4.1.3.

\subsubsection{4-Tx 4-Port TM2 vs TM4 rank-1 Throughput Differences at High and Low Antenna Correlation using SCME and EPA Channel Models}

Transmit diversity and spatial multiplexing transmission modes differ in their use of the antenna array. Transmit diversity, for instance, makes use of space and time diversity while transmitting symbols from each antenna. Transmit diversity typically performs better as antenna spacing increases, as the transmission mode implies. This improvement is not as obvious with spatial multiplexing modes which use transmit and receive beamforming where the antenna array elements generally need to be correlated for proper beamforming to occur. The transmission modes can clearly be affected in different ways if the environment changes, different channel models are used or the antenna configurations are changed.

Transmit diversity (TM2) is the fall-back mode for the more complex LTE transmission modes such as the spatial multiplexing modes, TM3, TM4 and TM6. Transmit diversity 
(TM2) and rank-1 closed-loop spatial multiplexing ${ }^{2}$ (TM4 rank-1) $)^{3}$ will be simulated using both the EPA extended-ITU model and the SCME. A comparison will be made between the throughput of TM2 and TM4 rank-1, using 4 transmitters and 4 antenna ports (4-Tx 4-port), to determine if the throughput of TM2 justifies falling back to TM2 or if spatial multiplexing outperforms TM2 under most conditions, while testing various antenna correlations.

In this section, the statistical models are used for benchmarking purposes since they have been widely used as industry tools for performance evaluation while the SCM, SCME and WINNER models are being adopted to model the more advanced spatial multiplexing modes where the throughput depends heavily on the spatial characteristics of the environment, the geometry of the antenna, field patterns, antenna correlation and polarization.

Unlike the more complex yet flexible spatial channel models, the distance between the antennas, the antenna patterns and the polarization are not defined based on geometry in the extended-ITU models. Therefore, 4-Tx 4-Port TM2 and TM4 rank-1 are compared at high and low antenna correlation when using the EPA channel model and $0.7 \lambda$ and $10 \lambda$ antenna spacings when using the SCME.

\section{TM2 vs TM4 Rank-1 Simulation Setup and Parameters}

For the performance comparison of 4-Tx 4-port TM2 and TM4 rank-1 in both SCME and extended-ITU channel, an LTE FDD downlink simulation of $20 \mathrm{MHz}$ of bandwidth at a carrier frequency of $2.6 \mathrm{GHz}$ is performed with $8 \mathrm{PRB}$ allocated to the UE. The carrier frequency is selected based on the nearly world-wide acceptance and deployment of LTE systems in the $2.6 \mathrm{GHz}$ frequency band. Transmit diversity mode TM2 and spatial multiplexing transmission mode TM4 rank-1 are evaluated with 4 BS transmitters and 4 antenna ports.

In the SCME "urban macro" simulations the BS is the origin and the UE is located 550 meters from the BS along the $x$-axis. The UE and BS antenna array heights are 1 and 30 meters above ground level (AGL). The UE is configured as 2 cross-polarized antennas as shown in figure 4.3a while the BS is 2 sets of cross-polarized antennas and the correlation is as described in the brief introduction in section 4.1.5.

The receiver is configured to use practical synchronization methods which make use of time and frequency offset estimation algorithms using the known synchronization and

\footnotetext{
${ }^{2}$ The rank of TM4 is limited to 1 to ensure a fair comparison between TM2 and TM4.

${ }^{3} \mathrm{TM} 4$ rank-1 is similar to TM6.
} 
reference signals available in the received sub-frames to acquire and compensate for timing and carrier frequency offsets as discussed in section 2.4.3. MMSE channel estimation is used at the receiver along with practical receiver front-end impairments ${ }^{4}$ enabled. OLLA is enabled and adjusts the UE suggested CQI at the eNodeB. The power of the CRS are boosted by $3 \mathrm{~dB}$ each which does not violate the maximum transmit power per antenna port since the power is borrowed from the RS of other ports that are not transmit on the current antenna port, as shown in figures 2.29, 2.30 and 2.31. Finally, typical processing delays are used at the UE and the BS which account for the time needed to run algorithms in the processors of the UE and the BS.

The reader is referred to table 4.3 for additional simulation details.

\section{4-Tx 4-port TM2 and TM4 rank-1 Simulation Results}

The expected theoretical throughput for a UE scheduled in 8 out of 100 PRB in the frequency domain is $4.968 \mathrm{Mbps}$, when using only single layer transmissions. The peak theoretical throughput calculated considering the number of PDSCH REs, the transport block size, the modulation order and the code rate limit is given in appendix C.1.2. The theoretical throughput calculation assumes single codeword transmissions for a fair comparison between TM2 and TM4 and also assumes the eNodeB allocates the 8 PRB outside of the central 6 PRB, where the PSS and SSS reside and the added overhead would reduce the throughput further.

A comparison of the simulated and theoretical peak throughput is available in table 4.4 and 4.5 for the highly correlated and uncorrelated cross-polarized antennas at $3 \mathrm{~km} / \mathrm{hr}$. The peak throughput comparison for both TM2 and TM4 rank-1 in table 4.4 and 4.5 are measured at $30 \mathrm{~dB}$ SNR in figures $4.4 \mathrm{a}$ and $4.4 \mathrm{~b}$. The percentage difference between the simulated and peak throughput shows that the simulated throughput is close to the expected peak throughput for TM4 rank-1 in all cases and for TM2 when using the EPA channel model. TM2 does not get as close to the theoretical peak throughput when using the SCME.

Tables 4.4 and 4.5 confirm that at sufficiently high SNR the simulator achieves results that are within a very small error margin of the expected throughput when using single layer closed-loop spatial multiplexing (TM4 rank-1).

\footnotetext{
${ }^{4}$ Practical receiver front end impairments include phase noise (1.5 $\mathrm{RMS}$ and bandwidth of $\left.10 \mathrm{kHz}\right), \mathrm{I}-\mathrm{Q}$ imbalance (magnitude of $0.5 \mathrm{~dB}$ and phase $1.5^{\circ}$ )
} 
Table 4.3: Simulation Parameters for 4-Tx 4-Port TM2 and TM4 Rank-1 Simulations using the SCME and EPA Channel Models

\begin{tabular}{|c|c|c|}
\hline Category & Parameter & Value \\
\hline \multirow{5}{*}{ System } & Bandwidth@Freq & $20 \mathrm{MHz}$ (100 PRBs)@2.6 GHz \\
\hline & Duplex mode & FDD \\
\hline & Sub-carrier spacing & $15 \mathrm{kHz}$ \\
\hline & Cyclic prefix & Normal CP $(4.7 \mu \mathrm{sec})$ \\
\hline & Simulation duration & 15,000 sub-frames \\
\hline \multirow{2}{*}{ BS antennas (SCME) } & Configurations & Fig $4.2 b, 4.2 \mathrm{c}$ \\
\hline & Spacing & $0.7 \lambda$ and $10 \lambda$ \\
\hline BS Antennas (EPA) & Correlation & High and low \\
\hline \multirow{4}{*}{ Channel } & Channel model & SCME urban macro and EPA \\
\hline & Angular spreading & Low (SCME only) \\
\hline & UE velocity & 3,30 and $120 \mathrm{~km} / \mathrm{hr}$ \\
\hline & SNR & Swept (-10 to $34 \mathrm{~dB})$ \\
\hline \multirow{3}{*}{ Resources/Scheduling } & Transmission modes & TM2 and TM4 rank-1 \\
\hline & UE resource blocks & $8 \mathrm{PRB}$ \\
\hline & OLLA & On \\
\hline \multirow{9}{*}{ Measurements/Reporting } & Channel estimation & MMSE \\
\hline & Synchronization & Practical $^{\mathrm{a}}$ \\
\hline & Receiver front-end & Practical $^{\mathrm{b}}$ \\
\hline & RS power boosting & $3 \mathrm{~dB}$ \\
\hline & CQI reporting interval & $5 \mathrm{msec}$ \\
\hline & CQI processing delay & $(\mathrm{UE}+\mathrm{BS}) 8 \mathrm{msec}$ \\
\hline & CQI sub-band group size & $8 \mathrm{PRB}$ \\
\hline & PMI group size & $8 \mathrm{PRB}$ \\
\hline & HARQ & 3 re-transmissions, 8 processes \\
\hline
\end{tabular}

\footnotetext{
${ }^{a}$ Practical synchronization uses known reference signals in the received sub-frames to estimate the offsets. Refer to section 4.1.5.

${ }^{b}$ Practical receiver front end impairments include phase noise (1.5 RMS and bandwidth of $\left.10 \mathrm{kHz}\right), \mathrm{I}-\mathrm{Q}$ imbalance (magnitude of $0.5 \mathrm{~dB}$ and phase $1.5^{\circ}$ ).
} 
Table 4.4: Simulated vs Theoretical Throughput for 4-Tx 4-port TM2 and TM4 rank-1 with Highly Correlated Cross-Polarized Antennas at $3 \mathrm{~km} / \mathrm{hr}$

\begin{tabular}{|c|c|c|c|c|}
\hline \multirow{3}{*}{$\begin{array}{c}\text { Transmission } \\
\text { Mode }\end{array}$} & \multicolumn{2}{|c|}{ EPA (High correlation) } & \multicolumn{2}{|c|}{ SCME $(0.7 \lambda$ spacing $)$} \\
\hline & Simulated & $\%$ Difference & Simulated & $\%$ Difference \\
\hline & Peak Throughput & vs Theory & Peak Throughput & vs Theory \\
\hline TM2 & 4.85 Mbps & $2.38 \%$ & 4.446 Mbps & $10.51 \%$ \\
\hline TM4 rank-1 & 4.934 Mbps & $0.68 \%$ & 4.924 Mbps & $0.86 \%$ \\
\hline
\end{tabular}

Table 4.5: Simulated vs Theoretical Throughput for 4-Tx 4-port TM2 and TM4 rank-1 with Uncorrelated Cross-Polarized Antennas at $3 \mathrm{~km} / \mathrm{hr}$

\begin{tabular}{|c|c|c|c|c|}
\hline \multirow{3}{*}{$\begin{array}{c}\text { Transmission } \\
\text { Mode }\end{array}$} & \multicolumn{2}{|c|}{ EPA (Low correlation) } & \multicolumn{2}{|c|}{ SCME (10 $\lambda$ spacing) } \\
\hline & Simulated & $\%$ Difference & Simulated & $\%$ Difference \\
\hline & Peak Throughput & vs Theory & Peak Throughput & vs Theory \\
\hline TM2 & 4.927 Mbps & $0.83 \%$ & 4.627 Mbps & $6.86 \%$ \\
\hline TM4 rank-1 & 4.994 Mbps & $0.52 \%$ & 4.936 Mbps & $0.64 \%$ \\
\hline
\end{tabular}


A summary of the throughput of both 4-Tx 4-port TM2 and TM4 rank-1, using correlated and uncorrelated cross-polarized antennas, at a SNR of $10 \mathrm{~dB}$ is given in table 4.6 for the EPA channel model and table 4.7 for the SCME.

Table 4.6: Throughput of 4-Tx 4-Port TM2 and TM4 rank-1 at $10 \mathrm{~dB}$ SNR (EPA)

\begin{tabular}{c|cc|cc}
\hline & \multicolumn{4}{c}{ Throughput (EPA) [Mbps] } \\
UE & High Antenna Correlation & Low Antenna Correlation \\
Velocity & TM2 & TM4 rank-1 & TM2 & TM4 rank-1 \\
\hline \hline $3 \mathrm{~km} / \mathrm{hr}$ & $1.64 \mathrm{Mbps}$ & $\mathbf{4 . 1 3} \mathbf{M b p s}$ & $2.41 \mathrm{Mbps}$ & $3.83 \mathrm{Mbps}$ \\
$30 \mathrm{~km} / \mathrm{hr}$ & $1.31 \mathrm{Mbps}$ & $\mathbf{2 . 5 0} \mathbf{M b p s}$ & $2.14 \mathrm{Mbps}$ & $1.54 \mathrm{Mbps}$ \\
$120 \mathrm{~km} / \mathrm{hr}$ & $1.29 \mathrm{Mbps}$ & $\mathbf{2 . 2 8} \mathbf{M b p s}$ & $2.08 \mathrm{Mbps}$ & $1.74 \mathrm{Mbps}$ \\
\hline
\end{tabular}

Table 4.7: Throughput of 4-Tx 4-Port TM2 and TM4 rank-1 at $10 \mathrm{~dB}$ SNR (SCME)

\begin{tabular}{|c|c|c|c|c|}
\hline \multirow{3}{*}{$\begin{array}{c}\text { UE } \\
\text { Velocity }\end{array}$} & \multicolumn{4}{|c|}{ Throughput (SCME) [Mbps] } \\
\hline & \multicolumn{2}{|c|}{ High Antenna Correlation $(0.7 \lambda)$} & \multicolumn{2}{|c|}{ 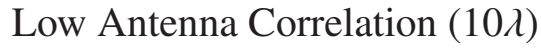 } \\
\hline & TM2 & TM4 rank-1 & TM2 & TM4 rank-1 \\
\hline $3 \mathrm{~km} / \mathrm{hr}$ & 1.90 Mbps & 3.49 Mbps & $1.94 \mathrm{Mbps}$ & 3.37 Mbps \\
\hline $30 \mathrm{~km} / \mathrm{hr}$ & 1.49 Mbps & 2.00 Mbps & $1.85 \mathrm{Mbps}$ & $1.86 \mathrm{Mbps}$ \\
\hline $120 \mathrm{~km} / \mathrm{hr}$ & $1.36 \mathrm{Mbps}$ & 1.87 Mbps & 1.40 Mbps & $1.75 \mathrm{Mbps}$ \\
\hline
\end{tabular}

When strictly comparing the throughput of TM2 using correlated and uncorrelated antenna elements, transmit diversity performs better when using uncorrelated antennas in both the EPA and SCME simulations. The opposite is true for TM4 rank-1 as seen in tables 4.6 and 4.7 and figures 4.4-4.6. The throughput of the highly correlated $(0.7 \lambda)$ cross-polarized antennas when using TM4 rank-1 surpasses that of the low (10 $\lambda)$ antenna correlation at all velocities at $10 \mathrm{~dB}$ SNR. The correlated antennas are beneficial to TM4 rank-1 since 4-Tx 4-port TM4 rank-1 uses beamforming, unlike TM2. 


\section{Low Velocity Simulation Results Discussion}

In the low velocity figures, figures $4.4 \mathrm{a}$ and $4.4 \mathrm{~b}$, for both uncorrelated and correlated cross-polarized antennas, the throughput of single layer closed-loop spatial multiplexing (TM4 rank-1) at $10 \mathrm{~dB}$ SNR is significantly higher than the fall-back mode transmit diversity (TM2). For the 8 PRB allocation, the throughput for TM4 rank-1 using correlated cross-polarized antennas is 4.13 and $3.49 \mathrm{Mbps}$ for EPA and SCME simulations at $3 \mathrm{~km} / \mathrm{hr}$ compared to 2.41 and 1.94 Mbps for the best antenna setup (low correlation) for TM2.

\section{Medium and High Velocity Results Discussion}

The medium and high velocity figures, figures 4.5 and 4.6, also show that TM4 rank-1 outperforms TM2 except when comparing the transmission modes using only low antenna correlations in the EPA simulations at 30 and $120 \mathrm{~km} / \mathrm{hr}$. In the medium and high velocity figures, the trend that TM4 rank-1 benefits from highly correlated antennas and TM2 benefits from uncorrelated antennas continues to be apparent.

\section{Gain of 4-Tx 4-Port TM4 rank-1 Using Correlated Antennas Over Other Modes and Antenna Correlations}

Since the highest throughput overall is achieved using 4-Tx 4-port TM4 rank-1 with correlated cross-polarized elements, this section looks at the gain of this mode over TM2 and other antenna correlations at a throughput of 2.5 Mbps. The gain of 4-Tx 4-port TM4 rank-1 over TM2 is summarized in table 4.8 .

4-Tx 4-port TM4 rank-1 with highly correlated antennas outperforms transmit diversity at all velocities and antenna correlations and spacings simulated. While both modes are capable of modulation and coding adaptation, TM4 rank-1 with correlated cross-polarized antennas adds beamforming using precoding in addition to the available polarization diversity. The added degree of freedom allows TM4 to direct the signal at the UE and increase the SNR. The result of the focused beam and increased SNR is that link adaptation is capable of choosing a higher modulation and coding scheme which results in a higher throughput than TM2.

The gain comparison in table 4.8 shows that closed-loop spatial multiplexing (TM4 


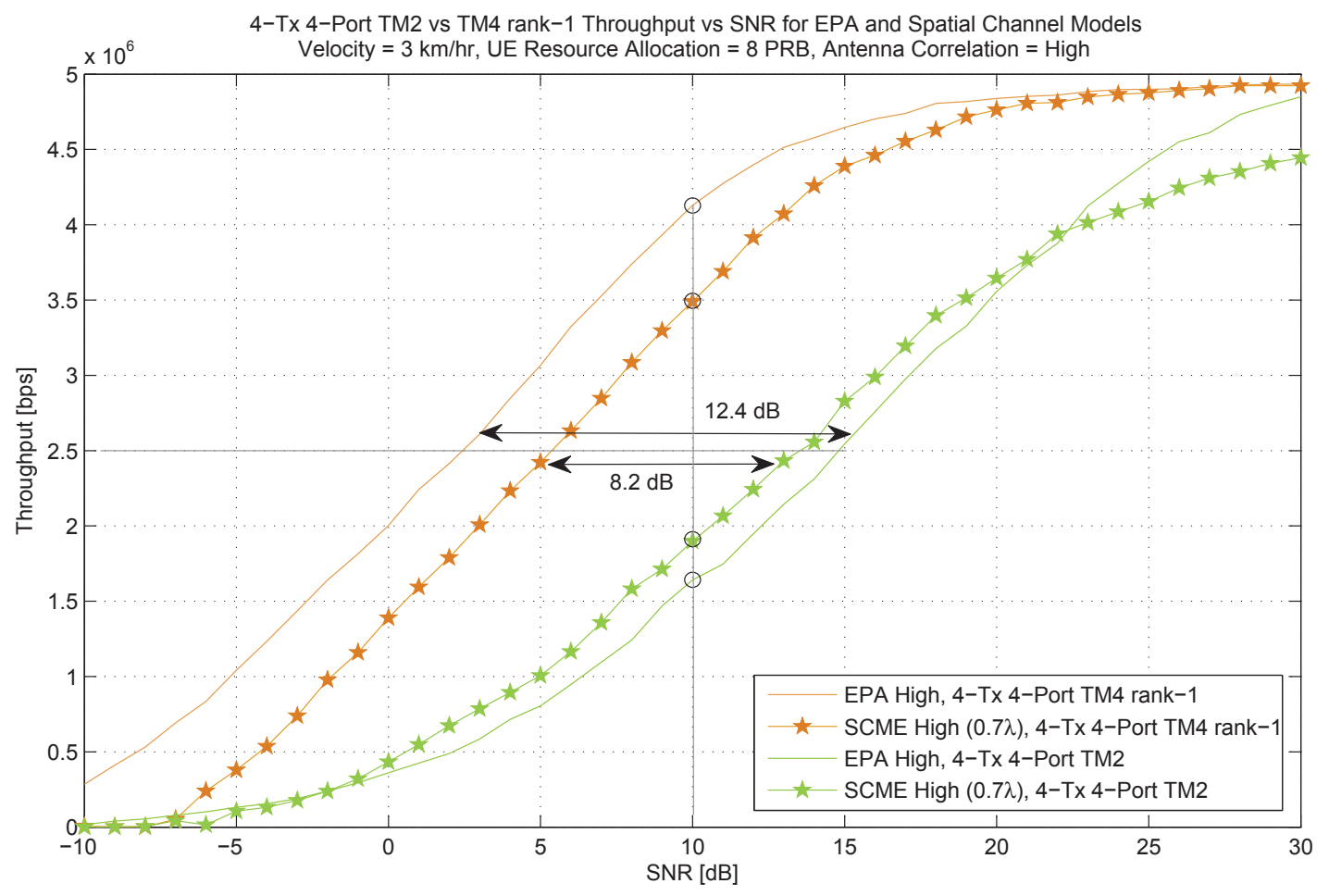

(a) 4-Tx 4-port TM2 and TM4 rank-1 EPA and SCME Throughput using Correlated Antennas

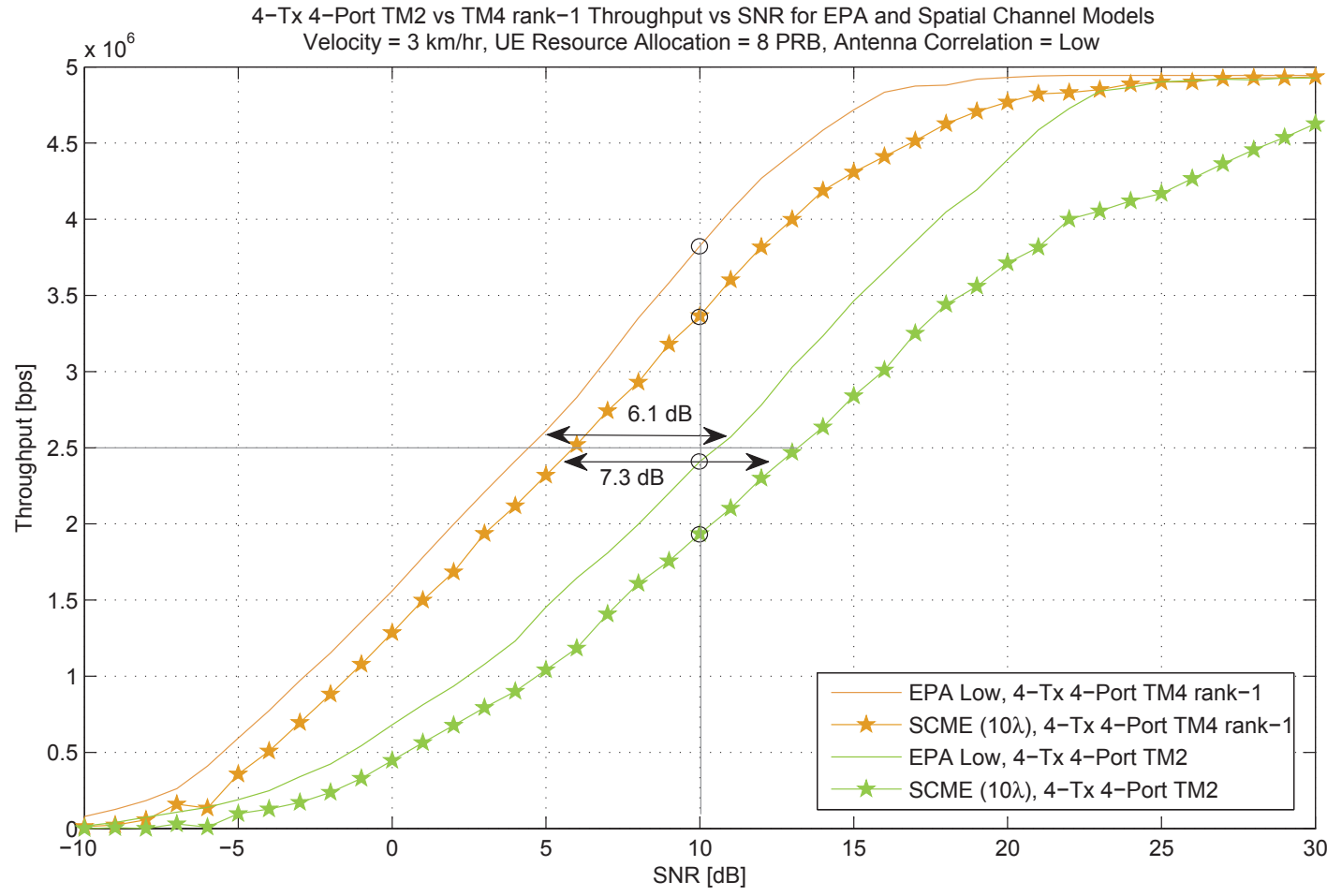

(b) 4-Tx 4-port TM2 and TM4 rank-1 EPA and SCME Throughput using Uncorrelated Antennas

Figure 4.4: 4-Tx 4-Port TM2 and TM4 rank-1 EPA and SCME Downlink Throughput using Cross-polarized Antennas at $3 \mathrm{~km} / \mathrm{hr}$ 


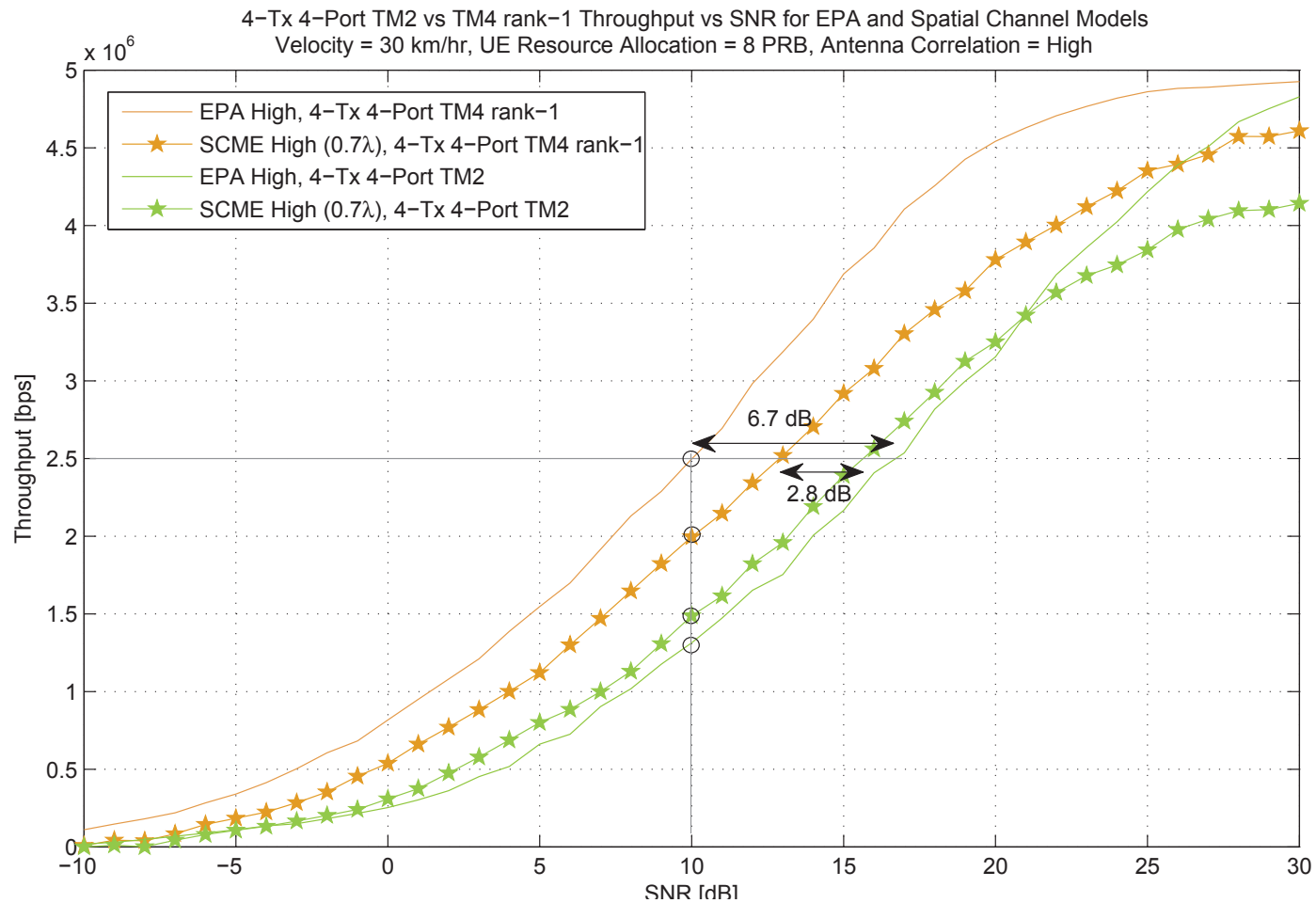

(a) 4-Tx 4-port TM2 and TM4 rank-1 EPA and SCME Throughput using Correlated Antennas

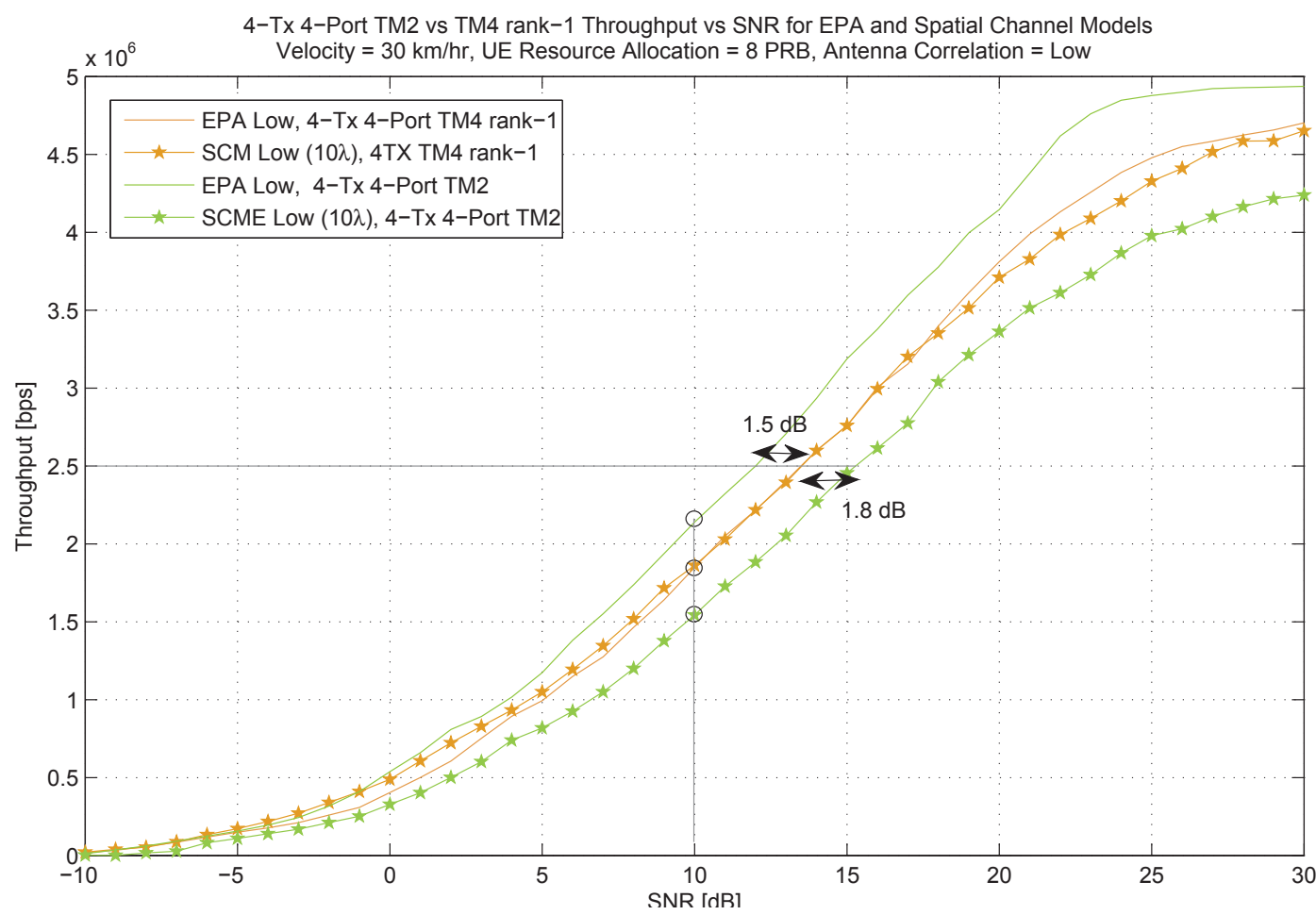

(b) 4-Tx 4-port TM2 and TM4 rank-1 EPA and SCME Throughput using Uncorrelated Antennas

Figure 4.5: 4-Tx 4-Port TM2 and TM4 rank-1 EPA and SCME Downlink Throughput using Cross-polarized Antennas at $30 \mathrm{~km} / \mathrm{hr}$ 


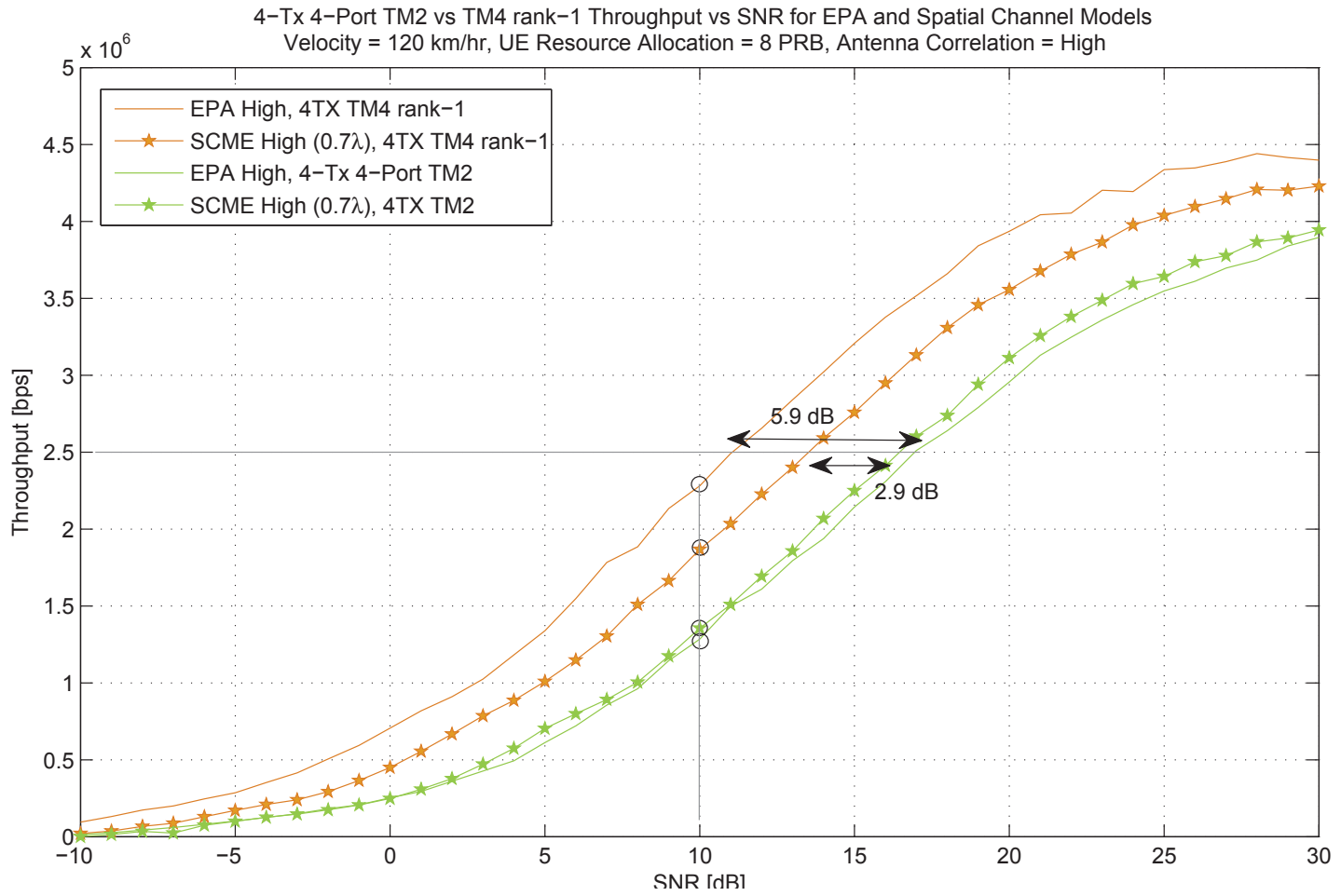

(a) 4-Tx 4-port TM2 and TM4 rank-1 EPA and SCME Throughput using Correlated Antennas

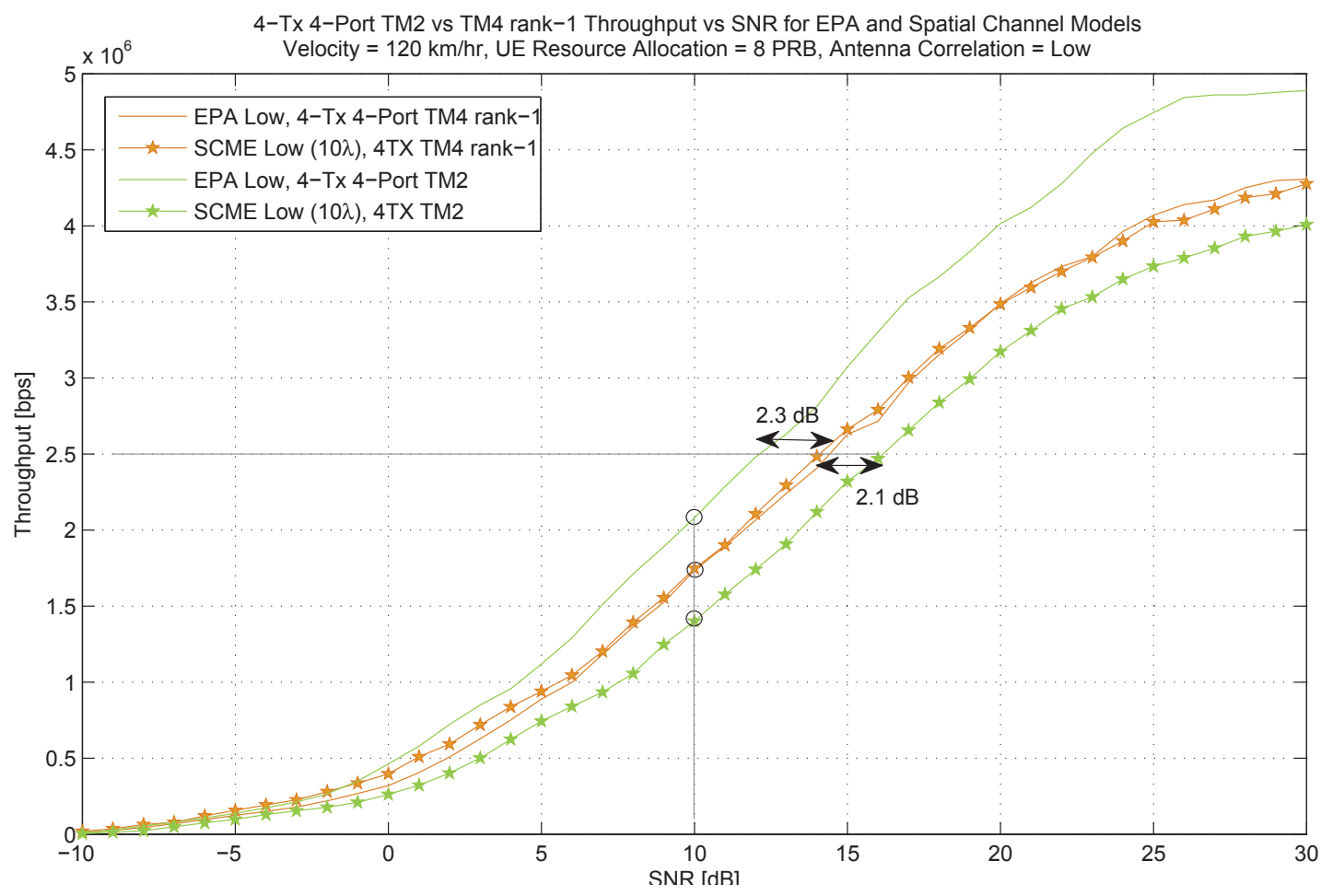

(b) 4-Tx 4-port TM2 and TM4 rank-1 EPA and SCME Throughput using Uncorrelated Antennas

Figure 4.6: 4-Tx 4-Port TM2 and TM4 rank-1 EPA and SCME Downlink Throughput using Cross-polarized Antennas at $120 \mathrm{~km} / \mathrm{hr}$ 
Table 4.8: Gain of 4-Tx 4-Port TM4 rank-1 Over Other Modes and Antenna Configurations at $2.5 \mathrm{Mbps}$

\begin{tabular}{c|cc|cc|cc|cc}
\hline & \multicolumn{3}{c}{ Gain of TM4 r-1 High Corr (EPA) } & \multicolumn{3}{c}{ Gain of TM4 r-1 (0.7 $\lambda$ ) (SCME) } \\
UE & \multicolumn{3}{|c|}{ Antenna Correlation } & \multicolumn{3}{c}{ Antenna Correlation } \\
High & \multicolumn{2}{c|}{ Low } & \multicolumn{2}{c}{ High $(0.7 \lambda)$} & \multicolumn{2}{c}{ Low $(10 \lambda)$} \\
Velocity & TM2 & TM4 r-1 & TM2 & TM4 r-1 & TM2 & TM4 r-1 & TM2 & TM4 r-1 \\
\hline \hline $3 \mathrm{~km} / \mathrm{hr}$ & $12.4 \mathrm{~dB}$ & Ref. & $8.1 \mathrm{~dB}$ & $2.0 \mathrm{~dB}$ & $8.2 \mathrm{~dB}$ & Ref. & $7.8 \mathrm{~dB}$ & $0.5 \mathrm{~dB}$ \\
$30 \mathrm{~km} / \mathrm{hr}$ & $6.7 \mathrm{~dB}$ & Ref. & $2.0 \mathrm{~dB}$ & $3.5 \mathrm{~dB}$ & $2.8 \mathrm{~dB}$ & Ref. & $2.4 \mathrm{~dB}$ & $0.6 \mathrm{~dB}$ \\
$120 \mathrm{~km} / \mathrm{hr}$ & $5.9 \mathrm{~dB}$ & Ref. & $1.1 \mathrm{~dB}$ & $3.4 \mathrm{~dB}$ & $2.9 \mathrm{~dB}$ & Ref. & $2.6 \mathrm{~dB}$ & $0.6 \mathrm{~dB}$ \\
\hline
\end{tabular}

rank-1) outperforms TM2 using correlated antennas, therefore, it is unlikely that transmit diversity would be needed.

The only case where TM2 is seen to outperform TM4 rank-1 is in the EPA simulation using uncorrelated antennas at 30 and $120 \mathrm{~km} / \mathrm{hr}$. Note that SCME does not lead to the same conclusion and based on SCME results 4-Tx 4-Port TM4 rank-1 would be sufficient in all scenarios (see results in section 4.1.5).

In addition to this conclusion, the gain of highly correlated TM4 rank-1 over uncorrelated TM2 is quite large $(8.1 \mathrm{~dB})$ at $3 \mathrm{~km} / \mathrm{hr}$ and significant $(2.0$ and $1.1 \mathrm{~dB})$ at 30 and 120 $\mathrm{km} / \mathrm{hr}$ as seen in table 4.8 . If using the highly correlated antennas, TM4 rank-1 is capable of much higher throughput at low velocity. TM2 may not be useful unless the channel conditions became harsh, for example when the UE is located near the cell edge and moving at medium or high velocity and the channel conditions more closely match the EPA model than the SCME.

Nevertheless, transmit diversity is still defined as a fall back mode in LTE due to its ability to offer good coverage and is used for special purpose channels, such as the control channels, that are critical and require decoding in the poorest conditions but the performance of spatial multiplexing with 4 transmitters and 4 ports is shown to be promising for all other conditions.

\section{EPA vs SCME Discussion}

The throughput performance of EPA and SCME simulations differ in figures 4.4, 4.5a and 4.6a. In general, the SCME throughput is lower than the EPA throughput results for TM4 
rank-1. The reader should note that the SCME is specific to the location and scenario that is randomly generated by the channel model and can lead to particular results. In the SCME, the randomly generated LSPs and scattering clusters are placed within the cell, as described in 2.3.7, and may generate less than optimal channel conditions at the UE location which may not be representative of the average performance across the cell.

Meanwhile the EPA model is an average scenario taken from many measurements in a typical cell environment. It is entirely possible that the average throughput of the EPA simulations is higher than the throughput of the SCME simulations at the particular drop location $(x, y, z)=(550,0,1)$ meters.

Factor that may cause the SCME throughput results to be lower than the EPA model:

- A location $(x, y, z)$ in the SCME drop that leads to a poor channel.

- A SCME "drop" and simulation "seed" that creates poor channel conditions for SCME simulations compared to EPA (eg. poor shadowing, NLOS).

- A SCME model that better models the correlation between antennas reducing the diversity compared to the fully uncorrelated elements used in the EPA low correlation MIMO channel correlation matrix.

- The crosstalk (high XPD) between polarized antenna may cause reduced diversity.

- A longer delay spread in the SCME simulation than the EPA may case higher frequency selectivity and lead to lower SCME throughput.

Nonetheless, the SCME simulations results can be very useful since they better represent a particular scenario, cell layouts, throughput at UE positions and can be used successfully to compare the performance of various antennas configurations, transmission modes, antenna polarizations and algorithms with accuracy.

In section 4.1.6, the promising results of TM4 limited to rank-1 will be built upon. Section 4.1.6 will further investigate spatial multiplexing modes, this time using OLSM and CLSM with multiple codewords (up to 2), 2 and 4 antenna elements, 2 types of elements polarizations and high and low antenna correlations.

\subsubsection{Number of Antennas and Antenna Port Simulations}

In this section, two spatial multiplexing schemes are compared, namely closed-loop (TM4) and open-loop spatial multiplexing (TM3). Since it was determined that the performance of spatial multiplexing provides gain over transmit diversity when using single codeword 
transmissions in section 4.1.5, transmit diversity (TM2) is not compared here since TM2 is limited to single codewords and the comparison in this section will allow multiple codewords (up to 2) to be transmit.

The goal of the following section is to determine if using 4 transmitters at the BS, along with spatial multiplexing transmission modes, does in fact provide higher throughput than the lower complexity 2 BS antenna configurations. This section will evaluate TM3 and TM4 using a larger set of BS antenna configurations including 2-Tx 2-port, 4-Tx 2Port and 4-Tx 4-port with both correlated and uncorrelated elements to determine the best antenna configuration.

\section{Number of Antennas and Antenna Port Simulation Parameters}

The transmission modes used in this LTE downlink throughput performance comparison are TM3 and TM4 with multiple transmission layers that permit up to two codewords to be sent simultaneously. The UE is allocated 50 PRBs within the $10 \mathrm{MHz}$ FDD spectrum at 2.6 $\mathrm{GHz}$, corresponding to allocating the full bandwidth to the UE. The transmission modes are evaluated using 2 and 4 base station (BS) transmitter antennas, with 2 and 4 antenna ports at low and high correlation.

The channel model used is the SCME with the urban macro scenario selected. The $\mathrm{BS}$ is again the origin and the UE is located 550 meters from the BS, this time at a $15^{\circ}$ angle from the $x$-axis (from bore sight). The UE and BS antenna array heights are 1 and 30 meters AGL as in previous simulations and as before the UE is configured as 2 crosspolarized antennas, as shown in figure 4.3a, while various BS antennas are evaluated. For the complete list of BS antennas used see table 4.9.

MMSE channel estimation is used at the receiver along with OLLA which allows the BS to adjust the UE suggested CQI for best performance. In these simulations, ideal synchronization is used and no receiver front-end impairments are applied. The power of the CRS, shown in figures 2.29, 2.30 and 2.31, are boosted by $3 \mathrm{~dB}$ each without violating maximum power constraints. In addition, practical processing delays of $4 \mathrm{msec}$ are used for both the BS and the UE and the CQI reporting period is set to $1 \mathrm{msec}$.

The UE velocities used in these simulations correspond to the standard 3GPP Doppler values (ie. 5 and $70 \mathrm{~Hz}$ ) converted to velocity in $\mathrm{km} / \mathrm{hr}$, as seen in table 4.9. For a complete list of the simulation parameters refer to table 4.9. 
Table 4.9: Simulation Parameters for the Antenna Configuration Simulations

\begin{tabular}{|c|c|c|}
\hline Category & Parameter & Value \\
\hline \multirow{5}{*}{ System } & Bandwidth@Freq & $10 \mathrm{MHz}(50 \mathrm{PRBs}) @ 2.6 \mathrm{GHz}$ \\
\hline & Duplex mode & FDD \\
\hline & Sub-carrier spacing & $15 \mathrm{kHz}$ \\
\hline & Cyclic prefix & Normal CP $(4.7 \mu \mathrm{sec})$ \\
\hline & Simulation duration & 15,000 sub-frames \\
\hline \multirow{3}{*}{ BS antennas } & Confiourations & Fig $4.1 \mathrm{a}, 4.1 \mathrm{~b}, 4.1 \mathrm{c}, 4.1 \mathrm{~d}$ \\
\hline & Cominguí autúts & Fig $4.2 \mathrm{a}, 4.2 \mathrm{~b}, 4.2 \mathrm{c}$ \\
\hline & Spacing & $0.7 \lambda$ and $10 \lambda$ \\
\hline \multirow{4}{*}{ Channel } & Channel model & SCME urban macro \\
\hline & Angular spreading & Low \\
\hline & UE velocity & 2.1 and $20.8 \mathrm{~km} / \mathrm{hr}$ \\
\hline & SNR & Swept (-10 to $30 \mathrm{~dB})$ \\
\hline \multirow{3}{*}{ Resources/Scheduling } & Transmission mode(s) & TM3 rank-1/2 vs TM4 rank-1/2 \\
\hline & UE resource blocks & All PRBs \\
\hline & OLLA & On \\
\hline \multirow{9}{*}{ Measurements/Reporting } & Channel estimation & MMSE \\
\hline & Synchronization & Ideal \\
\hline & Receiver front-end & Ideal $^{\mathrm{a}}$ \\
\hline & RS power boosting & $3 \mathrm{~dB}$ \\
\hline & CQI reporting interval & $1 \mathrm{msec}$ \\
\hline & CQI processing delay & $4 \mathrm{msec}(\mathrm{UE}), 4 \mathrm{msec}(\mathrm{BS})$ \\
\hline & CQI sub-band group size & $8 \mathrm{PRB}$ \\
\hline & PMI group size & Wideband \\
\hline & HARQ & 3 re-transmissions, 8 processes \\
\hline
\end{tabular}

a Ideal receiver front-end does not model radio imperfections (eg. I-Q imbalance, phase noise, quantization, automatic gain control (AGC)). 


\section{Number of Antennas and Antenna Port Simulation Results}

The simulation results for low UE velocity $(2.1 \mathrm{~km} / \mathrm{hr})$ are presented in figures 4.7 and 4.8 , while the medium velocity $(20.8 \mathrm{~km} / \mathrm{hr})$ results are presented in figures 4.9 and 4.10 .

The figures are presented in order of overall highest to lowest throughput observed in the typical usable SNR range of 0 to $15 \mathrm{~dB}$ SNR. In this SNR range 4-Tx 4-port TM4 provides gain over other antenna configurations and modes at low UE velocity but begins to show signs of some throughput degradation at $20.8 \mathrm{~km} / \mathrm{hr}$ due to CQI and PMI age and reporting frequency. Nonetheless, the following discussion shows that 4 transmitters and 4 antenna ports can improve the performance of the system when using TM4.

\section{Low Velocity Regime}

The 4-Tx 4-port cross-polarized antenna configuration with spacing $0.7 \lambda$ offers the best performance overall for CLSM (TM4) at low velocity. The gain of 4-Tx 4-port TM4 over 2-Tx 2-port TM4 and TM3 is $1.64 \mathrm{~dB}$ at 25Mbps. A summary of the gain of 4-Tx 4-port TM4 over competing antenna configurations and modes is shown in table 4.10.

Table 4.10: Gain of 4 Transmitters 4-Port TM4 Over Other Number of Antennas at 25 Mbps at a UE Velocity of $2.1 \mathrm{~km} / \mathrm{hr}$

\begin{tabular}{ccc}
\hline $\begin{array}{c}\text { Antenna } \\
\text { Configuration }\end{array}$ & $\begin{array}{c}\text { Gain of 4-Tx 4-Port TM4 } \\
\text { Over the Next Best Antenna Config. }\end{array}$ & $\begin{array}{c}\text { Gain of 4-Tx 4-Port TM4 } \\
\text { Over 4-Tx 4-Port TM3 }\end{array}$ \\
\hline \hline Cross-Pol 0.7 & $1.64 \mathrm{~dB}^{\mathrm{a}}$ & $3.26 \mathrm{~dB}$ \\
Co-Pol 10 $\lambda$ & $0.10 \mathrm{~dB}^{\mathrm{b}}$ & $1.77 \mathrm{~dB}$ \\
Cross-Pol 10 & $0.57 \mathrm{~dB}^{\mathrm{c}}$ & $1.89 \mathrm{~dB}$ \\
Co-Pol 0.7 $\lambda^{\mathrm{e}}$ & $1.23 \mathrm{~dB}^{\mathrm{d}}$ & $6.58 \mathrm{~dB}$ \\
\hline
\end{tabular}

a 2 Tx 2 Port TM4 and TM3 rank-1/2 using cross-polarized antennas.

b 2 Tx 2 Port TM4 and TM3 rank-1/2 using co-polarized antennas with spacing $10 \lambda$.

c 2 Tx 2 Port TM4 and TM3 rank-1/2 using co-polarized antennas.

d 2Tx 2Port TM4 rank-1/2 using co-polarized antennas with spacing $0.7 \lambda$.

e Highly correlated co-polarized antennas are not recommend for use due to the poor performance of both 2 and 4 antennas and both spatial multiplexing transmission modes evaluated.

In terms of peak throughput, the 2-Tx 2-port TM4 curve peaks just above the 4-Tx 4-port TM4 curve. The small peak throughput difference of 2-Tx 2-port TM4 over 4-Tx 


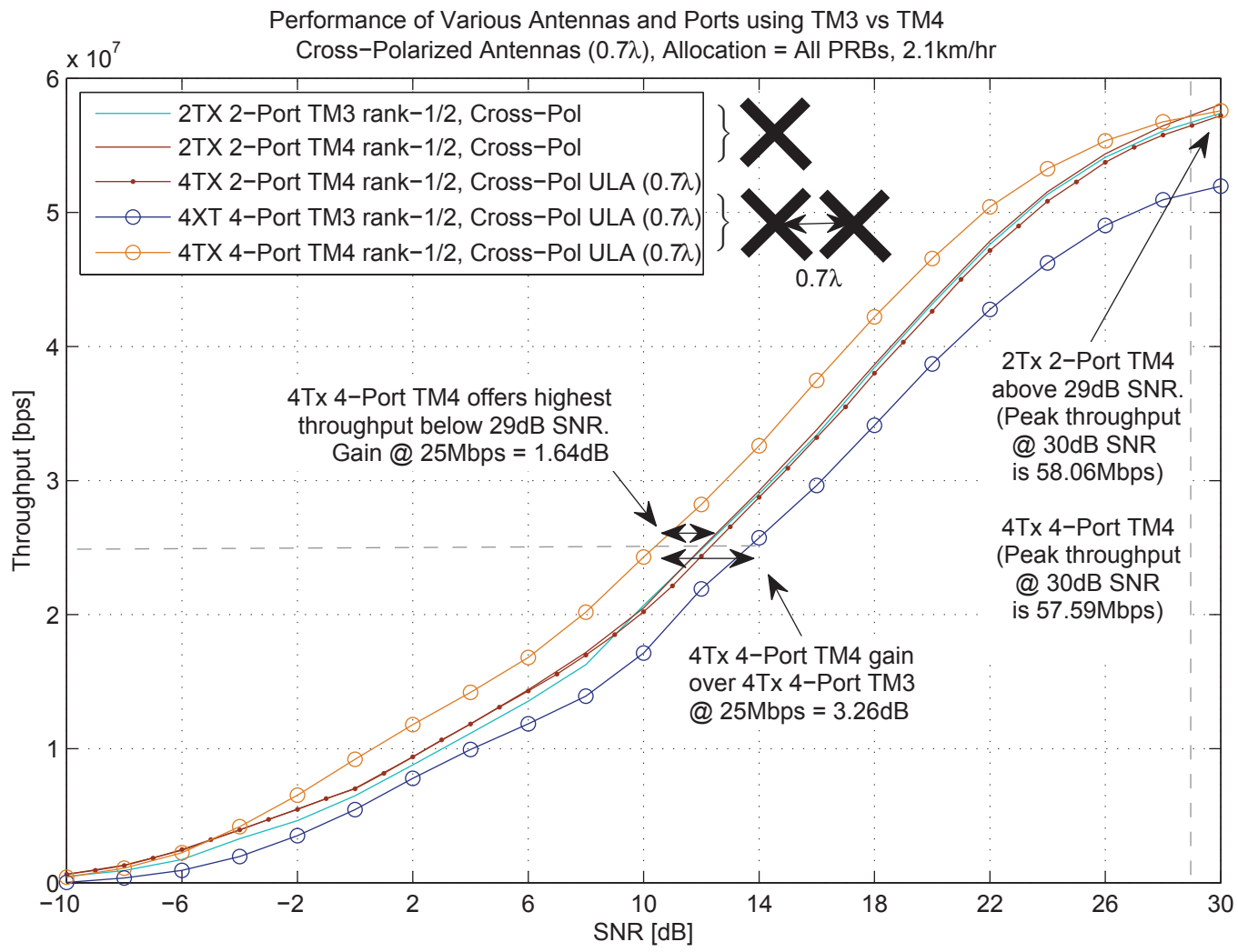

(a) Correlated $(0.7 \lambda)$ Cross-polarized Antennas $(2.1 \mathrm{~km} / \mathrm{hr})$

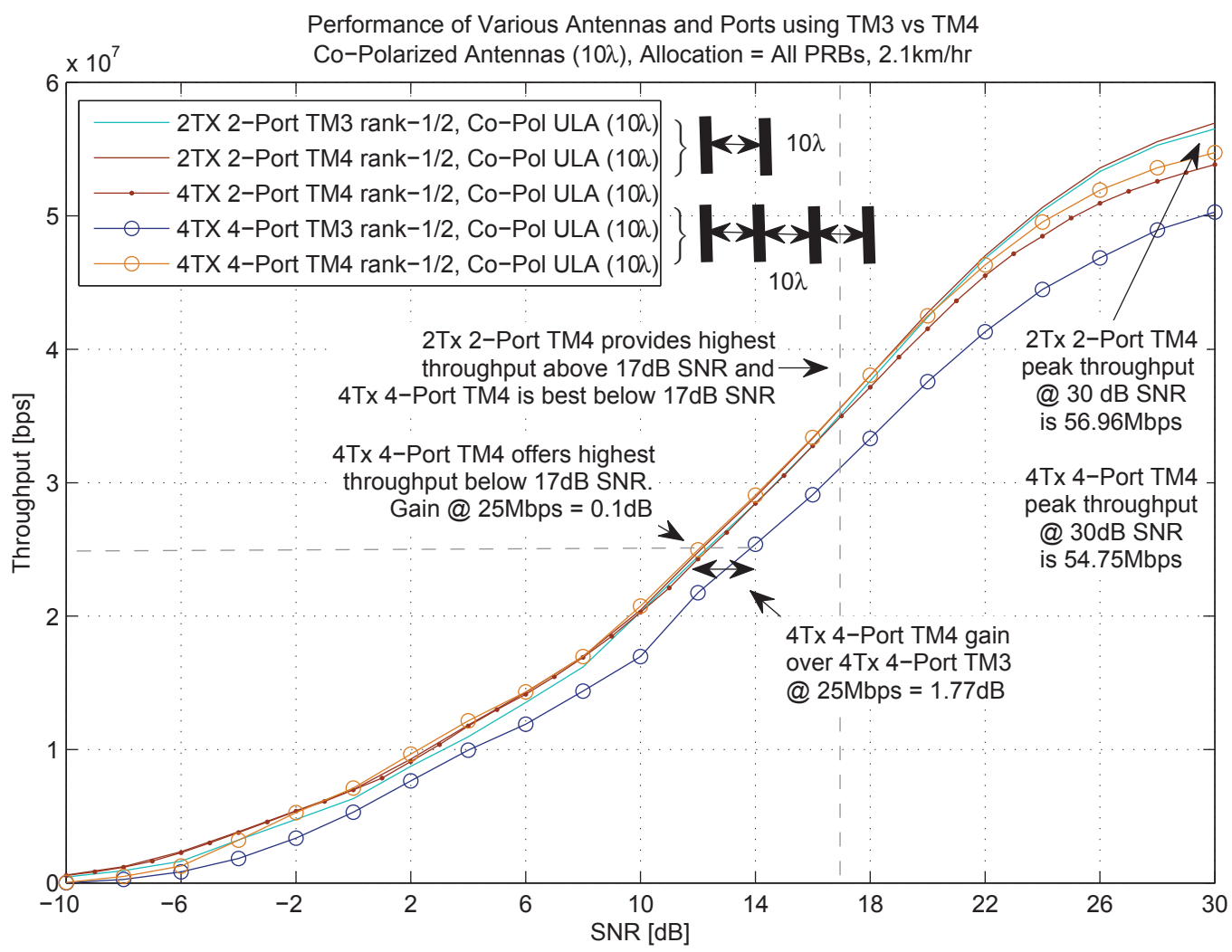

(b) Uncorrelated (10ג) Co-polarized Antennas $(2.1 \mathrm{~km} / \mathrm{hr})$

Figure 4.7: LTE DL Performance versus Number of Antennas and Ports at Low Velocity at a UE Velocity of $2.1 \mathrm{~km} / \mathrm{hr}$ 


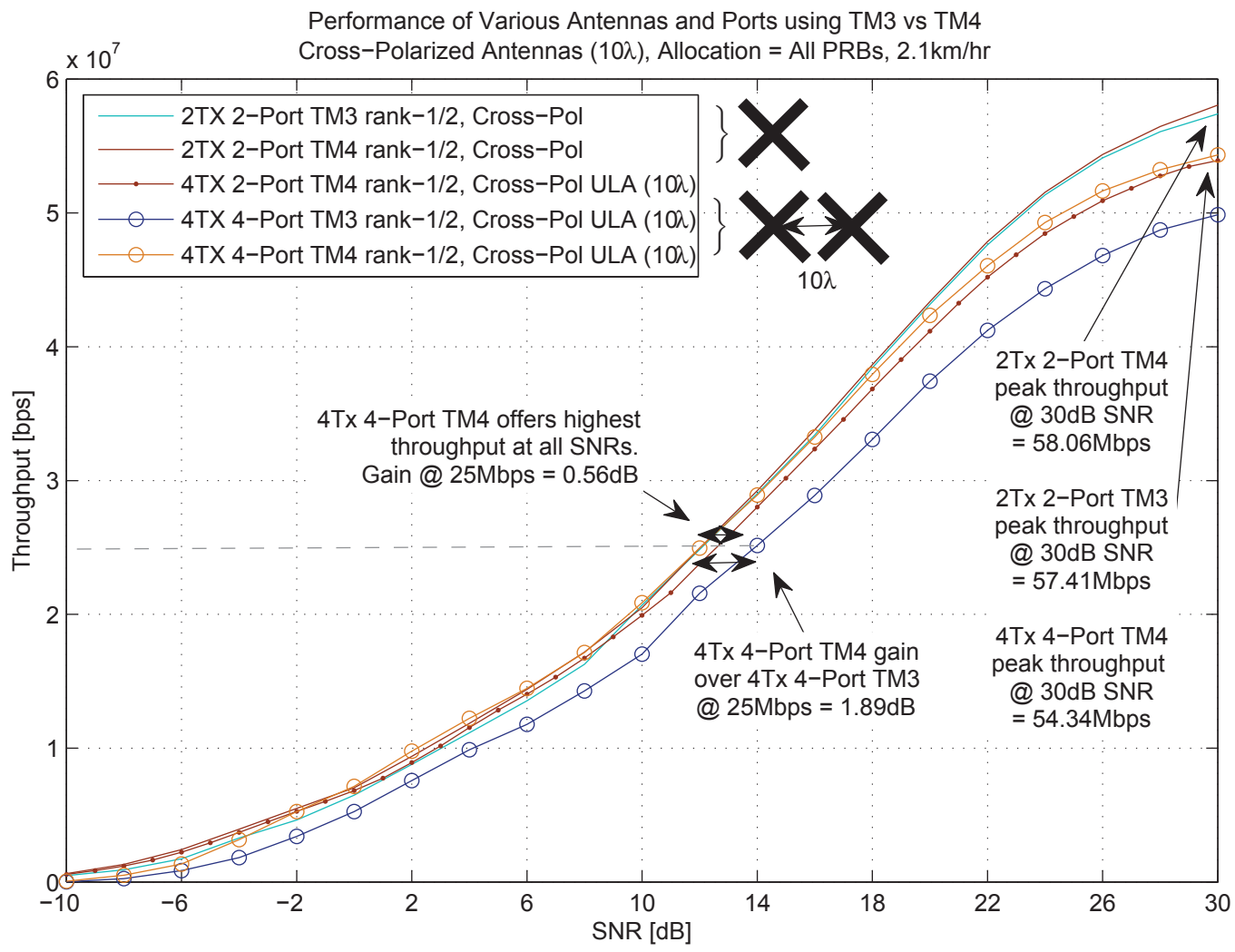

(a) Uncorrelated (10ג) Cross-polarized Antennas (2.1 km/hr)

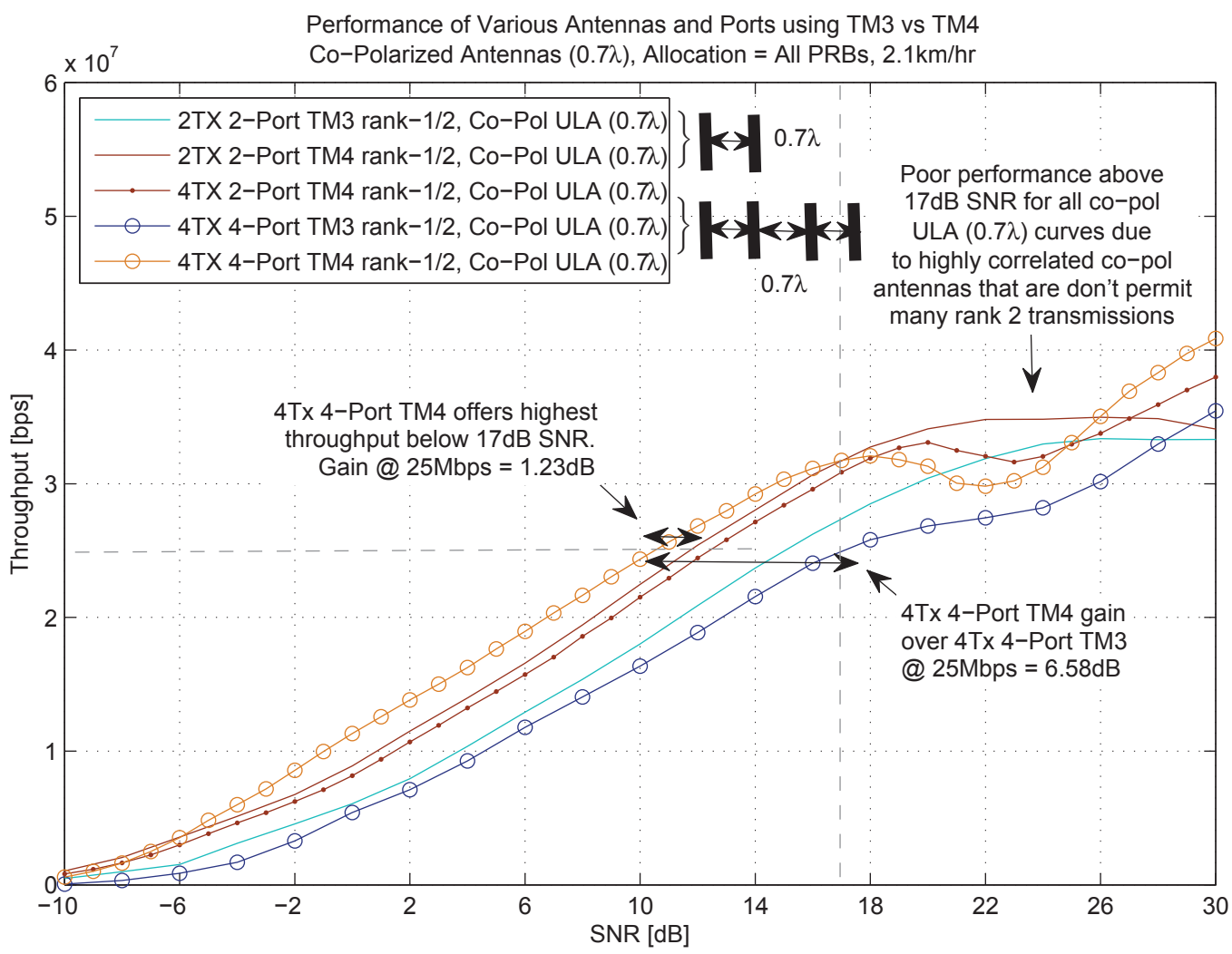

(b) Correlated $(0.7 \lambda)$ Co-polarized Antennas $(2.1 \mathrm{~km} / \mathrm{hr})$

Figure 4.8: LTE DL Performance versus Number of Antennas and Ports at a UE Velocity of $2.1 \mathrm{~km} / \mathrm{hr}$ 
4-port TM4 is $0.047 \mathrm{Mbps}$ in the correlated cross-polarized antenna case (figure 4.7a) and is due to the lower reference signal overhead when using 2 antenna ports compared to 4 antenna ports. Since the peak difference is in a narrow SNR range and also quite small, 4Tx 4-port TM4 can be assumed to be the best overall when using correlated cross-polarized BS antennas.

When comparing the throughput difference of 4-Tx 4-port between the correlated crosspolarized antennas (figure 4.7a) and the uncorrelated co-polarized antennas (figure 4.7b) the throughput of 4-Tx 4-port TM4 is lower in the uncorrelated co-polarized antennas case. The gain of 4-Tx 4-port becomes a mere $0.1 \mathrm{~dB}$ over the 2-Tx 2-port TM4 and TM3 curves at $25 \mathrm{Mbps}$ since their throughput does not change much. This indicates that 4-Tx 4-port TM4 does not perform as well with the uncorrelated co-polarized antennas. The polarization diversity of cross-polarized antennas provides an additional dimension of diversity, namely polarization diversity, that 4 co-polarized elements does not.

The peak throughput difference between the 2-Tx 2-port TM4 and TM3 and the 4-Tx 4-port TM4 curves at $30 \mathrm{~dB}$ SNR is $2.21 \mathrm{Mbps}$ in the uncorrelated co-polarized antenna case (figure 4.7b). As the SNR increases beyond $17 \mathrm{~dB}$, in the uncorrelated co-polarized antenna comparison in figure 4.7b, the lower RS overhead of 2-Tx 2-port TM3 and TM4 causes the throughput of these two transmission modes to peak above the peak throughput of 4-Tx 4-port TM4, which has a larger RS overhead.

The uncorrelated cross-polarized antenna comparison shown in figure 4.8a shows similar throughput curves as the uncorrelated co-polarized curves in figure $4.7 \mathrm{~b}$. The agreement between the uncorrelated curves indicates that the polarization alone does not have much of an effect on the throughput of the system. The higher throughput of 4-Tx 4-port TM4 in figure $4.7 \mathrm{a}$ is due to the combination of cross-polarized antennas and an array spacing of $0.7 \lambda$. The closer spacing is required for optimal beamforming. The closely spaced cross-polarized antennas matched with 4-Tx 4-port TM4 permits optimal beamforming to increase the SNR seen at the UE along with polarization diversity which helps to increase the number of information streams in order to send multiple codewords.

\section{Low Throughput in the High Correlation Co-Polarized Antennas Case}

It is obvious from figure $4.8 \mathrm{~b}$ that the highly correlated $(0.7 \lambda)$ co-polarized array ${ }^{5}$ and channel is unable to support rank-2 transmissions due to the very low throughput seen

\footnotetext{
${ }^{5}$ Highly correlated co-polarized antennas are not recommend for use due to the poor performance of both 2 and 4 antennas and both spatial multiplexing transmission modes evaluated.
} 
above $17 \mathrm{~dB}$ SNR where rank-2 transmission would normally take place. The previously discussed antenna configurations provide higher rank channel matrices more often due to the greater level of isolation between the antenna elements due to polarization diversity and/or element spacing. The rank- 2 transmissions allows the system throughput to continue to increase as seen in the results for the cross-polarized BS array (figures 4.7a and 4.8a) and the results for the uncorrelated co-polarized BS array (figure 4.7b). The correlated antennas may allow beamforming to occur but the real gain occurs when the antennas are sufficiently uncorrelated and the channel conditions permit spatial multiplexing leading to multiple codewords being sent simultaneously across the channel, effectively doubling the throughput in the medium-high SNR region.

\section{Medium Velocity Regime}

At $20.8 \mathrm{~km} / \mathrm{hr}$, a small amount of throughput degradation is visible when looking specifically at the 4-Tx 4-port cross-polarized antennas spaced by $0.7 \lambda$. The peak throughput of cross-polarized 4-Tx 4-port TM4, measured at $30 \mathrm{~dB}$ SNR, drops from 57.59 Mbps at $2.1 \mathrm{~km} / \mathrm{hr}$ (figure $4.7 \mathrm{a}$ ) to $52.51 \mathrm{Mbps}$ at $20.8 \mathrm{~km} / \mathrm{hr}$ (figure $4.9 \mathrm{a}$ ). However, the correlated 4-Tx 4-port cross-polarized antennas still outperform other antenna configurations when using TM4 as seen in table

In the next sub-section 4.1.6, the 2 transmitter configurations will be left out and only 2 antenna topologies will be compared using only 4 transmitter 4-port antenna configurations for the polarization and antenna element correlation simulations.

Table 4.11: Gain of 4 Transmitters 4-Port TM4 Over Other Number of Antennas at 25 Mbps at a UE Velocity of $20.8 \mathrm{~km} / \mathrm{hr}$

\begin{tabular}{ccc}
\hline $\begin{array}{c}\text { Antenna } \\
\text { Configuration }\end{array}$ & $\begin{array}{c}\text { Gain of Best Mode } \\
\text { Over the Next Best Antenna Config. }\end{array}$ & $\begin{array}{c}\text { Gain of 4-Tx 4-Port TM4 } \\
\text { vs 4-Tx 4-Port TM3 }\end{array}$ \\
\hline \hline Cross-Pol 0.7 & $0.36 \mathrm{~dB}$ (4-Tx 4-Port TM4) & $2.71 \mathrm{~dB}$ (4-Tx 4-Port TM4) \\
Co-Pol 10 $\lambda$ & $0.66 \mathrm{~dB}$ (2-Tx 2-Port TM3) & $0.72 \mathrm{~dB}$ (4-Tx 4-Port TM3) \\
Cross-Pol 10 $\lambda$ & 0.54 dB (4-Tx 2-Port TM4) & $0.5 \mathrm{~dB}$ (4-Tx 4-Port TM3) \\
Co-Pol 0.7 $\lambda^{\mathrm{a}}$ & $0.5 \mathrm{~dB}$ (4-Tx 4-Port TM4) & 8.76 dB (4-Tx 4-Port TM4) \\
\hline
\end{tabular}

a Highly correlated co-polarized antennas are not recommend for use due to the poor performance of both 2 and 4 antennas and both spatial multiplexing transmission modes evaluated. 


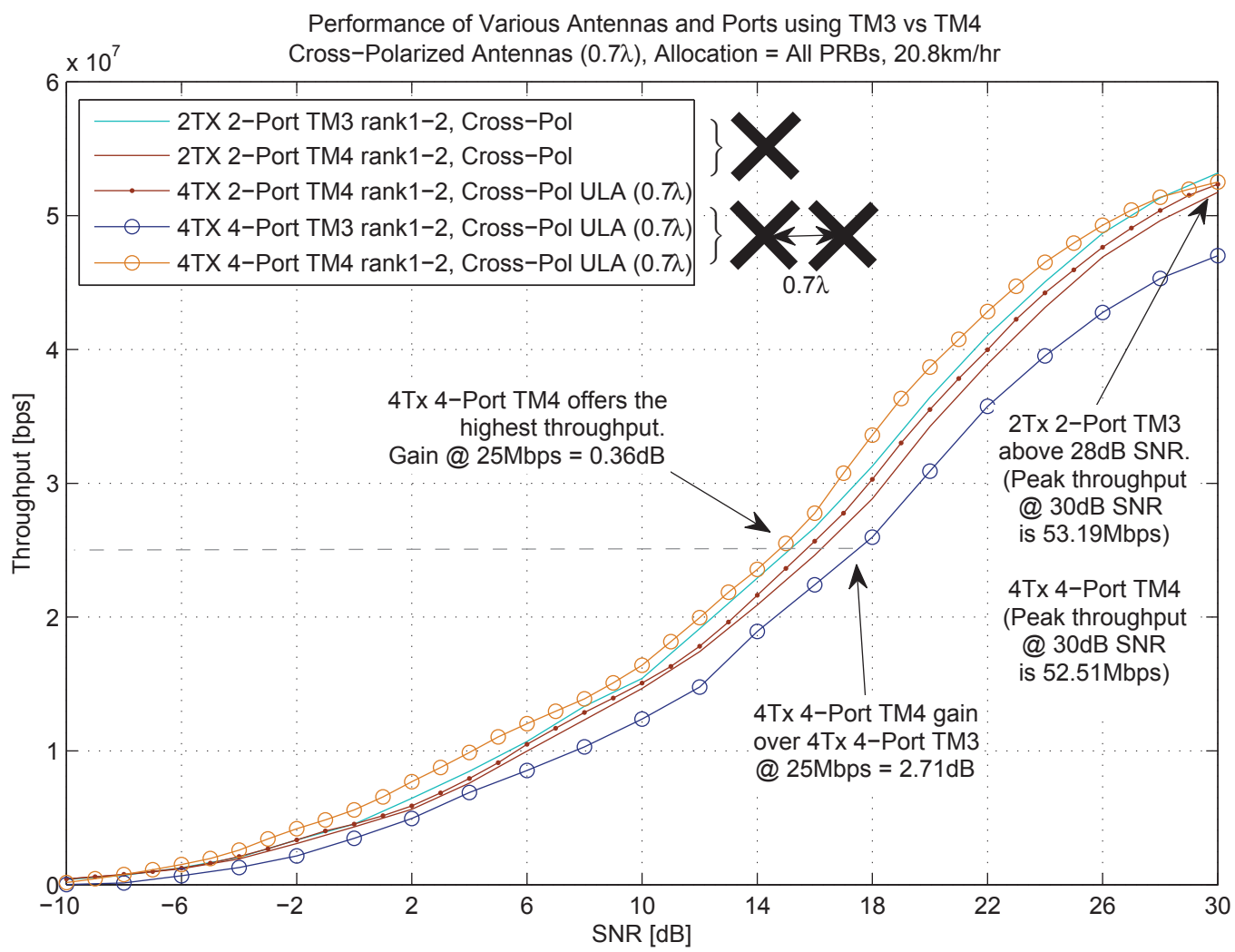

(a) Correlated $(0.7 \lambda)$ Cross-polarized Antennas $(20.8 \mathrm{~km} / \mathrm{hr})$

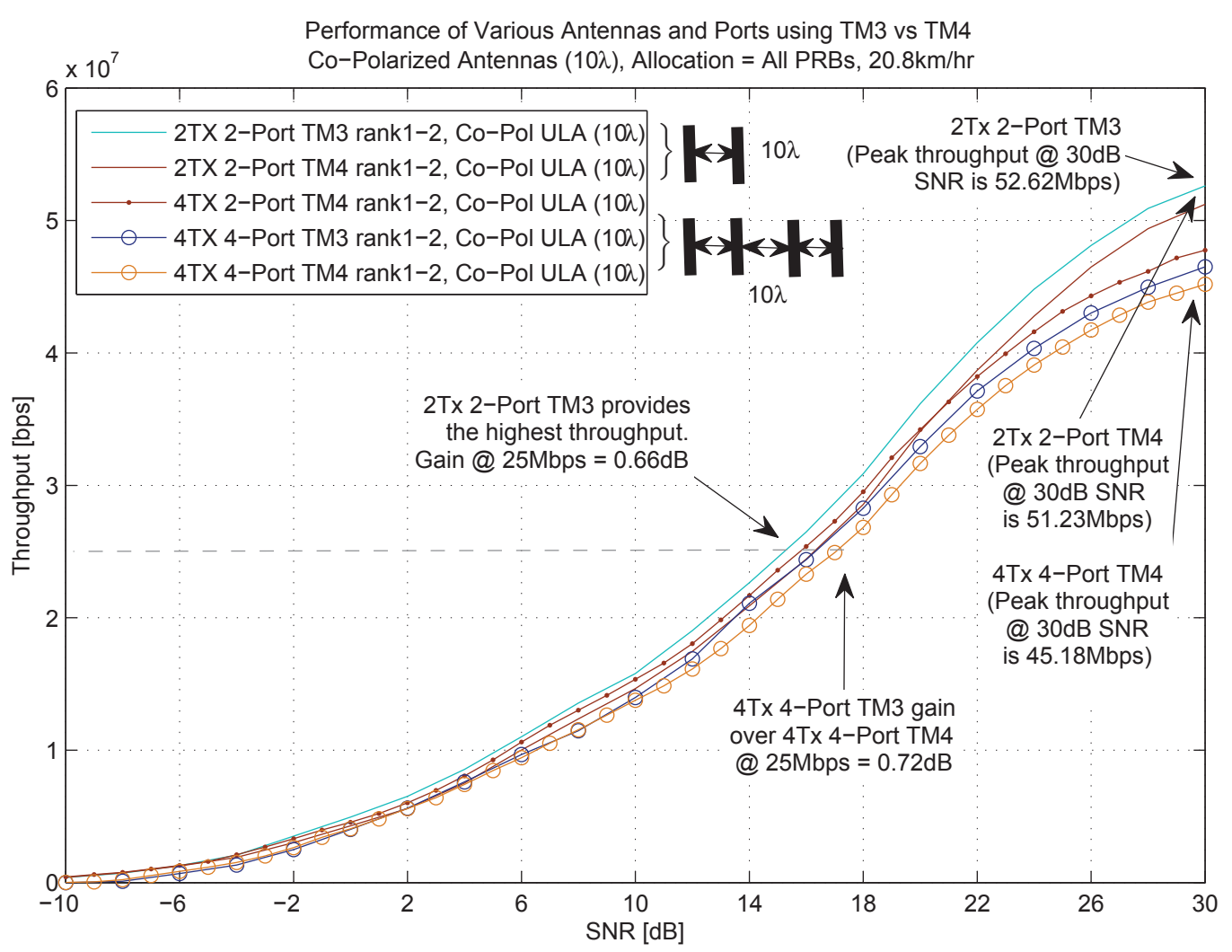

(b) Uncorrelated (10ג) Co-polarized Antennas (20.8 km/hr)

Figure 4.9: LTE DL Performance versus Number of Antennas and Ports at a UE Velocity of $20.8 \mathrm{~km} / \mathrm{hr}$ 


\section{TM3 vs TM4 Discussion}

While TM4 typically performs better with 4 transmitters and 4-ports, TM3 on the other hand, performs better with the 2-Tx 2-port antenna configuration. 4-Tx 4-port is beneficial to TM4 because the reliable and fine resolution PMI feedback received from the UE every 1 msec provides TM4 with an optimal beam pattern and gain over TM3. TM3 however is unable to provide the same gain as TM4 since the PMI feed-back is not used. Instead, 4-Tx 4-port TM3 uses CDD with a cycling precoder which causes the beam pattern to "sweep" the antenna array coverage area, as discussed in 2.5.3. The effects of the cycling precoder in TM3 is a less than optimal precoder selection during most of the simulation resulting in reduced throughput. Similarly, in the 2 antenna port cases, TM3 may be less than optimal since a single fixed precoder is used. The reason 2 port TM3 performs better than 4 port TM3 is the reduced number of reference signals when using 2 antenna ports which permits higher throughput due to less overhead. In the case of 4-Tx 4-port TM3 the additional RS on antenna ports $\{2,3\}$ are not used to select the optimal beam pattern (no PMI feedback), therefore, the added overhead when using four transmitters and four ports appears to not be justified when using TM3.

In some cases TM3 can have an advantage over TM4. TM3 was introduced into the LTE standard due to its reduced feedback overhead on the uplink. If only 2 transmitters or 2 ports are permitted, TM3 can provide comparable throughput to 2-Tx 2-port or 4Tx 2-port TM4 at low velocity and at $20.8 \mathrm{~km} / \mathrm{hr} 2$-Tx 2-port TM3 performs better than 2-Tx 2-port and 4-Tx 2-port TM4. The lack of the PMI feed-back means TM3 is less dependent on channel measurement and CSI reporting than 2-Tx 2-port or 4-Tx 2-port TM4. TM3's lack of the dependence on the PMI reports is beneficial as the UE velocity increases because of the rapid variations in the channel. At high UE velocity, the rapid variations of the channel cause the CSI to become aged if not frequently transmit and promptly used by the eNodeB for transmission. Refer to section 4.3 for a more in-depth discussion and simulation relating to the topic of UE velocity and feed-back reporting on closed and open-loop spatial multiplexing.

\section{CSI Feed-back Rate}

The simulation results use a feed-back reporting period of $1 \mathrm{msec}$. In practice, the feedback reporting rate would be lower (anywhere from 40 or $20 \mathrm{msec}$ down to $10 \mathrm{msec}$ in a-periodic reporting mode depending on the eNodeB scheduler implementation). A lower practical feed-back rate could reasonably be expected to reduce the throughput of TM4, in 


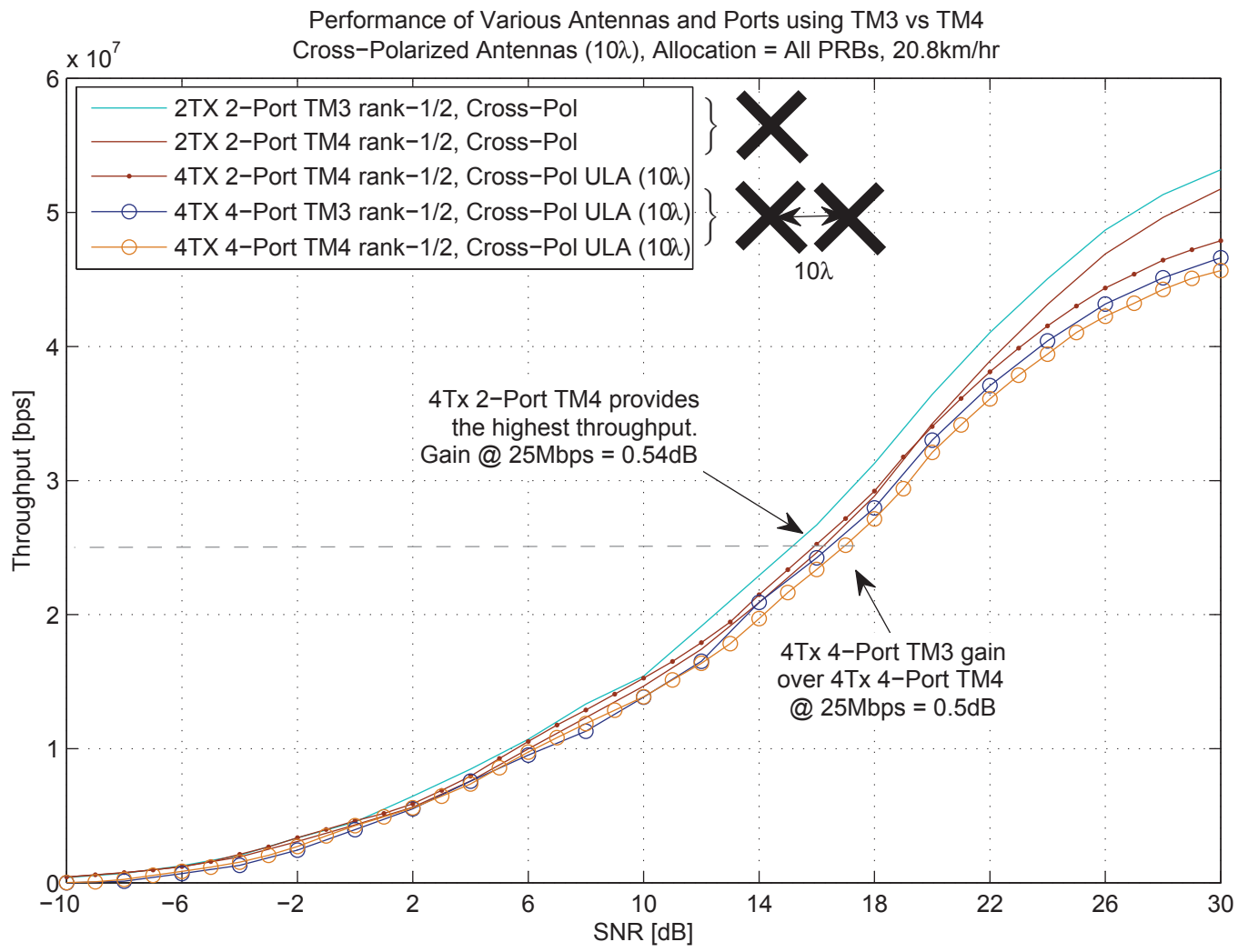

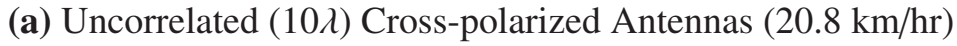

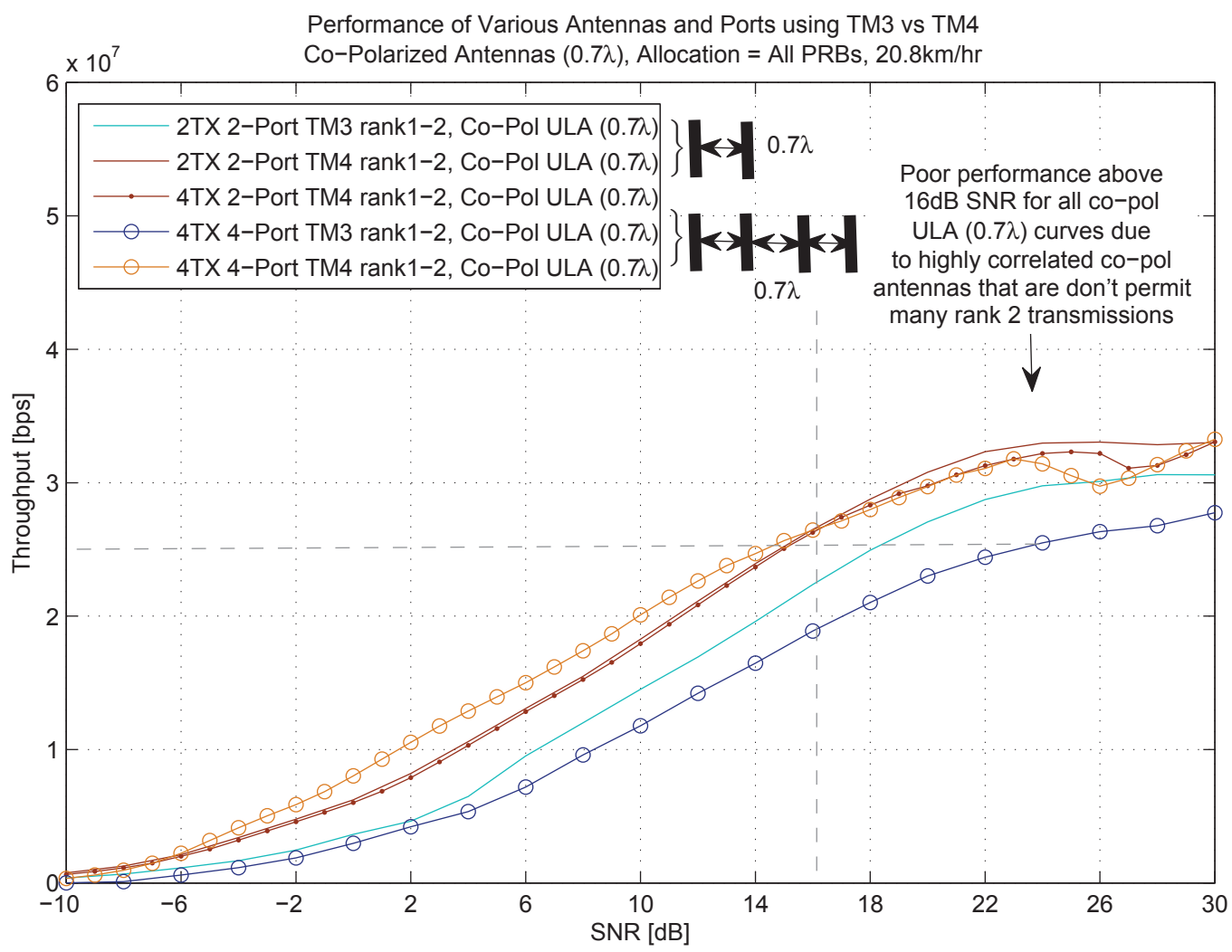

(b) Correlated $(0.7 \lambda)$ Co-polarized Antennas $(20.8 \mathrm{~km} / \mathrm{hr})$

Figure 4.10: LTE DL Performance versus Number of Antennas and Ports at a UE Velocity of $20.8 \mathrm{~km} / \mathrm{hr}$ 
relation to TM3, especially at medium and high velocities where the channel is changing quickly and the reported PMI is no longer reliable at the time of its use in the eNodeB. Feed-back reporting and UE velocity is further investigated in section 4.3.

\section{Best Antenna Configurations Comparison}

Table 4.12 is a summary of the throughput for various antenna configurations and transmission modes measured at $14 \mathrm{~dB}$ SNR. The best antenna configuration when using TM3 is the 2-Tx 2-port cross-polarized antennas providing up to $29.0 \mathrm{Mbps}$ and 22.9 Mbps at 2.1 and $20.8 \mathrm{~km} / \mathrm{hr}$ when using the cross-polarized configuration. Two transmitters always provide the highest throughput at $14 \mathrm{~dB}$ SNR for TM3 no matter the antenna polarization configuration. This can be related to the lower overhead when using 2 antenna ports instead of 4 antenna ports and a lower importance placed on beamforming.

In the case of TM4, the highest throughput is achieved with the 4-Tx 4-port correlated cross-polarized antennas. TM4 and the 4 cross-polarized transmitters are able to make use of beamforming and polarization diversity to achieve $32.6 \mathrm{Mbps}$ and 23.6 Mbps at 2.1 and $20.8 \mathrm{~km} / \mathrm{hr}$.

Table 4.12: Throughput of Best Antenna Configuration at $14 \mathrm{~dB}$ SNR $(2.1 \mathrm{~km} / \mathrm{hr})$

\begin{tabular}{|c|c|c|c|c|}
\hline \multirow{2}{*}{$\begin{array}{c}\text { Antenna } \\
\text { Configuration }\end{array}$} & \multicolumn{2}{|c|}{$2.1 \mathrm{~km} / \mathrm{hr}$} & \multicolumn{2}{|c|}{$20.8 \mathrm{~km} / \mathrm{hr}$} \\
\hline & TM3 & TM4 & TM3 & TM4 \\
\hline \multirow{2}{*}{ Cross-Pol $0.7 \lambda$} & 29.0 Mbps & 32.6 Mbps $\sqrt{ }$ & 22.9 Mbps & 23.6 Mbps $\sqrt{ }$ \\
\hline & 2-Tx 2-Port & 4-Tx 4-Port & 2-Tx 2-Port & 4-Tx 4-Port \\
\hline \multirow{2}{*}{ Co-Pol $10 \lambda$} & $28.4 \mathrm{Mbps}$ & 29.1 Mbps & $22.7 \mathrm{Mbps}$ & 21.7 Mbps \\
\hline & 2-Tx 2-Port & 4-Tx 4-Port & 2-Tx 2-Port & 4-Tx 2-Port \\
\hline \multirow{2}{*}{ Cross-Pol $10 \lambda$} & 29.0 Mbps & 29.3 Mbps & 22.9 Mbps & 21.5 Mbps \\
\hline & 2-Tx 2-Port & 2-Tx 2-Port & 2-Tx 2-Port & 4-Tx 2-Port \\
\hline \multirow{2}{*}{ Co-Pol $0.7 \lambda^{\mathrm{a}}$} & $23.7 \mathrm{Mbps}$ & $29.2 \mathrm{Mbps}$ & 19.6 Mbps & $24.7 \mathrm{Mbps}$ \\
\hline & $2 \mathrm{~T} \times 2$ Port & 4-Tx4-Port & 2 Tx 2 Port & 4-Tx 4-Port \\
\hline
\end{tabular}

${ }^{a}$ Highly correlated co-polarized antennas are not recommend for use due to the poor performance of both 2 and 4 antennas and both spatial multiplexing transmission modes evaluated. 


\section{Transmitter, 4 Port Comparison}

Figure 4.11 is a comparison of the best 4 transmitter and 4 antenna port curves for both open and closed-loop spatial multiplexing at 2.1 and $20.8 \mathrm{~km} / \mathrm{hr}$. Figure 4.11 highlights the fact that TM4 performs best with the closely spaced cross-polarized antennas in both cases, while the best quad transmitter antenna configuration for TM3 depends on the UE velocity.

\section{4-Tx 4-Port TM4 and 2-Tx 2-Port TM3 Comparison}

Finally, the curves in figure 4.12 are a comparison of the best antenna configuration for each transmission mode.

The 4-Tx 4-port closely spaced cross-polarized antennas remain the optimal BS antenna configuration for TM4. In the case of open-loop spatial multiplexing, TM3 performs best with 2 transmitters and the cross-polarized antennas are shown to have a small amount of gain over the 2 antenna uncorrelated (10ג) ULA. The figures also show that, not surprisingly, the throughput of 2-Tx 2-port TM3 approaches that of 4-Tx 4-port TM4 at $20.8 \mathrm{~km} / \mathrm{hr}$ since TM4 begins to suffer throughput degradation due to the quickly changing channel, the processing delays and the aged PMI reports that do not affect TM3.

\section{Antenna Configurations Conclusion}

Multiple antenna configurations, polarizations and correlations were evaluated in this section using both open and closed-loop spatial multiplexing. The antenna configurations were shown to be dependent on the transmission modes in use, or vice-versa, which implies that the RAN system designer must consider multiple factors, including the available equipment, UE velocity and available transmission modes (implementation complexity) when specifying the physical layer design. Ideally, 4 BS transmitters would be available and configured in the closely spaced cross-polarized configuration which was shown to provide the highest throughput. TM4 would be the transmission mode of choice when using 4 transmitters at the base station.

When using TM3, only one pair of cross-polarized transmitters are needed in order to achieve the best TM 3 throughput. The benefits of using only two antennas are that antenna hardware cost could be lower, the size of the deployed antennas would be smaller and the system complexity would also be lower. 


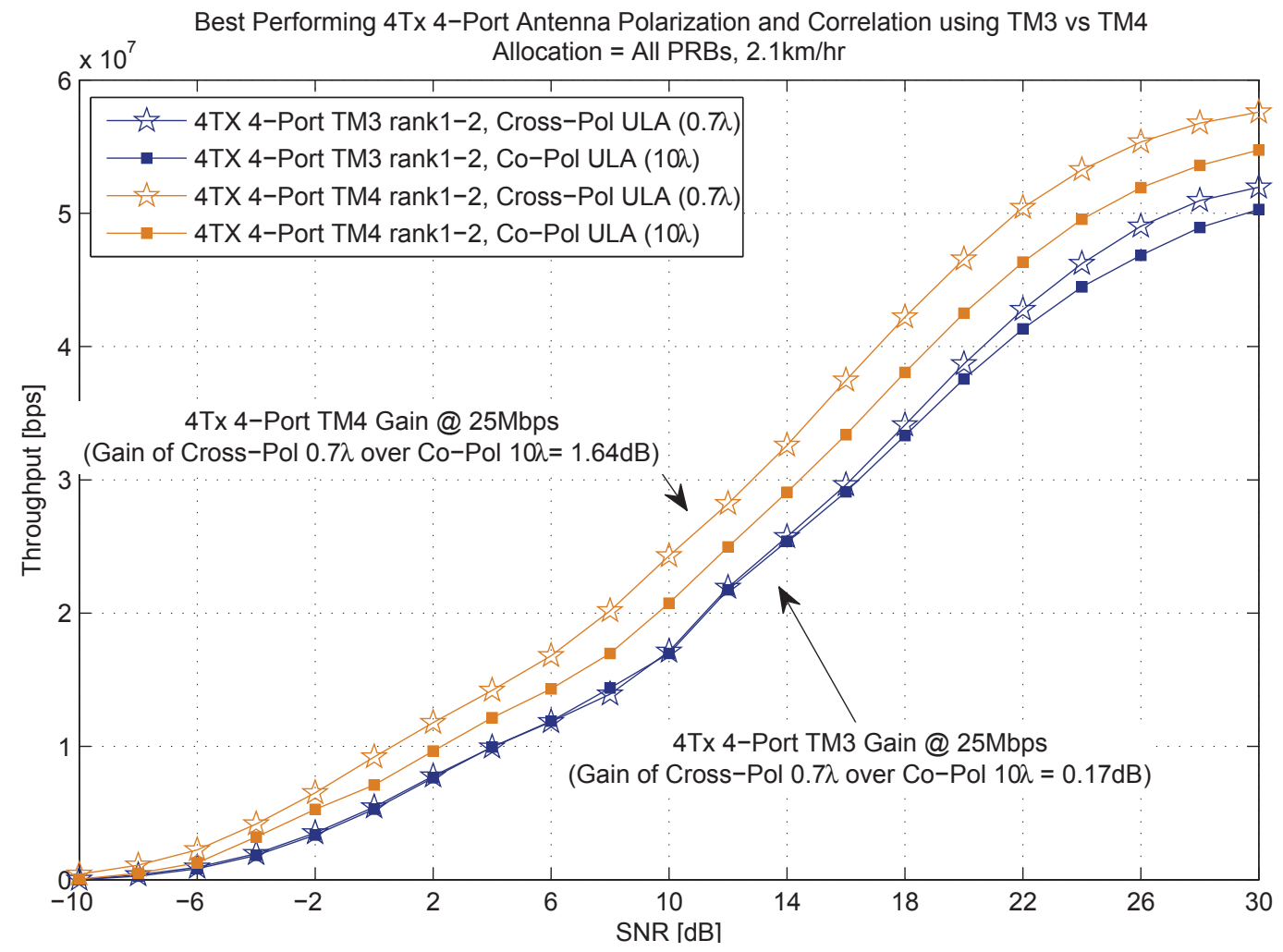

(a) Cross-polarized vs Co-Polarized Antennas $(2.1 \mathrm{~km} / \mathrm{hr})$

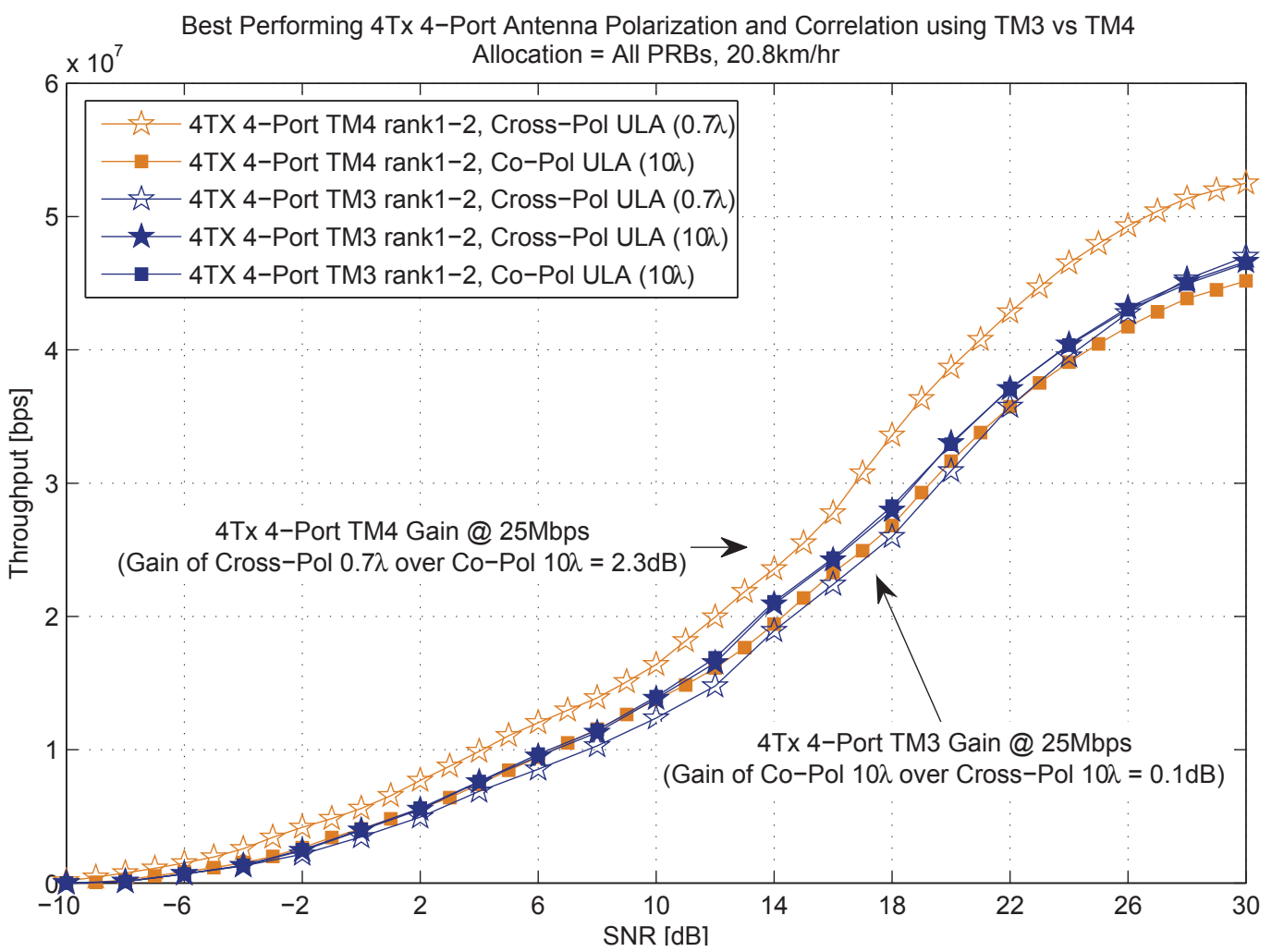

(b) Cross-polarized vs Co-Polarized Antennas $(20.8 \mathrm{~km} / \mathrm{hr})$

Figure 4.11: Comparison of 4-Tx 4-Port Polarization and Antenna Correlation 


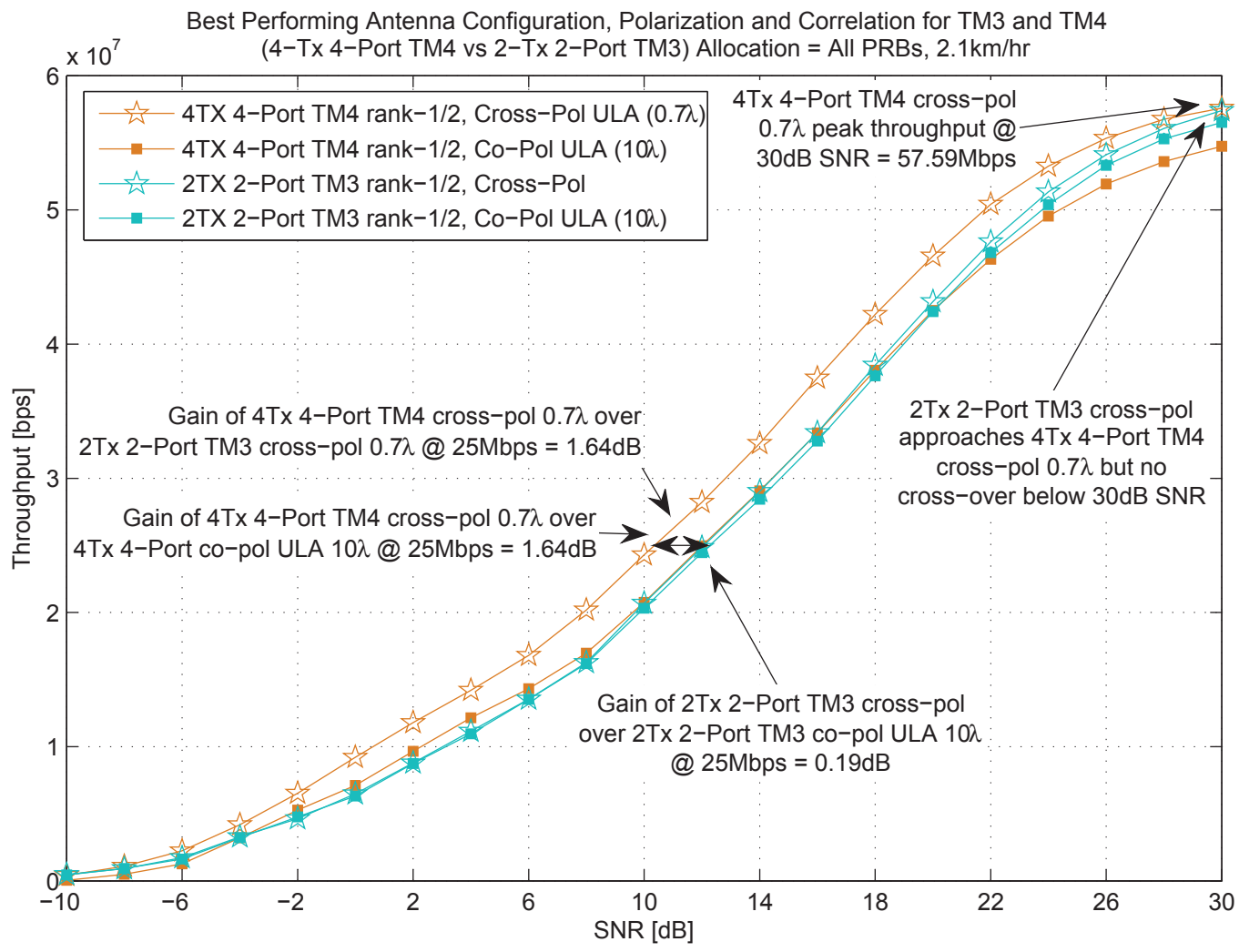

(a) Best Performing Antenna Configuration for Each Transmission Mode $(2.1 \mathrm{~km} / \mathrm{hr})$

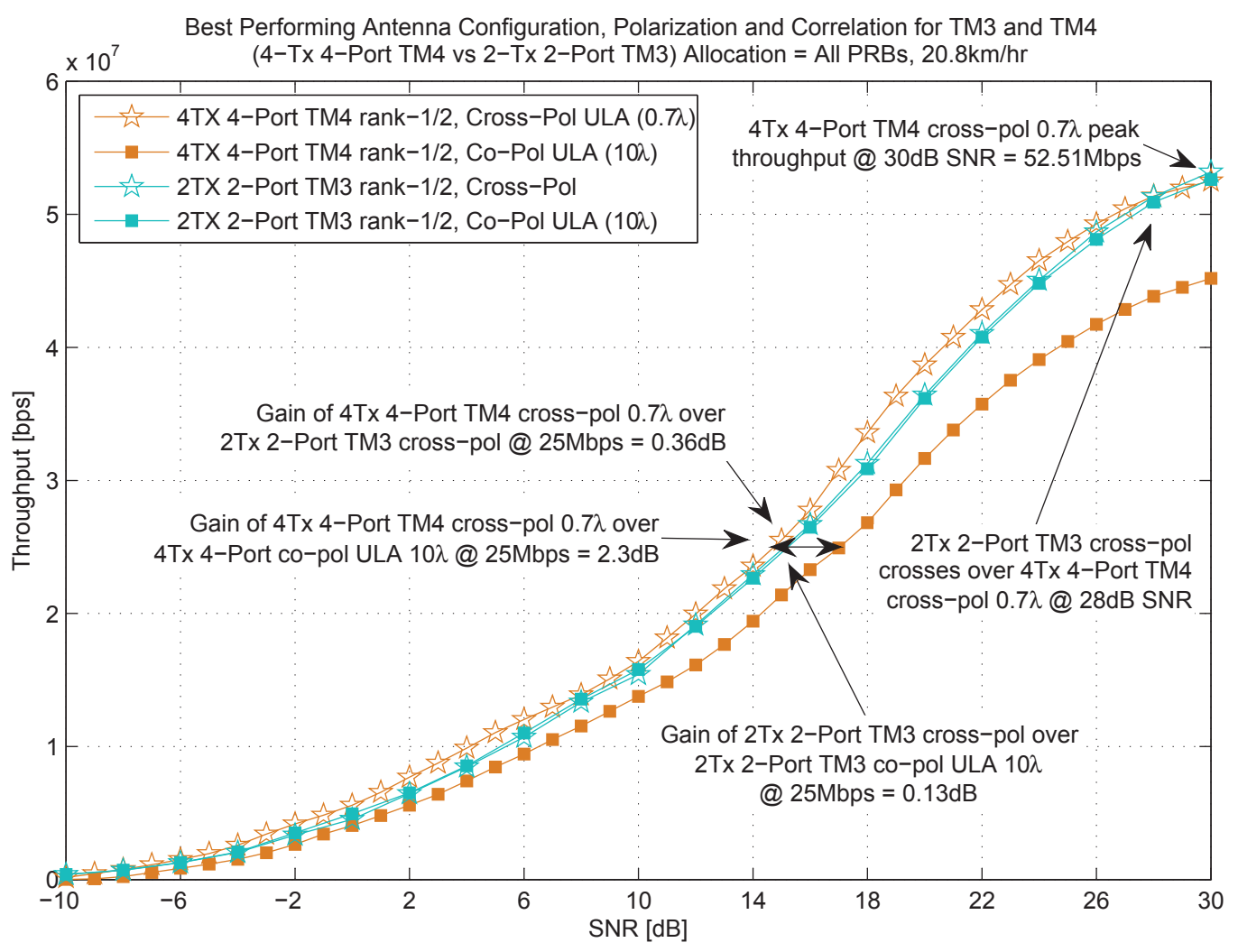

(b) Best Performing Antenna Configuration for Each Transmission Mode (20.8 km/hr)

Figure 4.12: Best Performing Antenna Configuration for Each Transmission Mode 
Finally, it is not necessary to switch between the transmission modes if 4-Tx 4-port TM4 with closely spaced $(0.7 \lambda)$ cross-polarized antennas is used UE, according to the simulations presented in this thesis. On the other hand, if two antenna ports are used, transmission mode switching could be beneficial

Since four eNodeB antennas were shown to provide gain over two eNodeB antennas, when using TM4, only the best performing antenna configuration (4-Tx 4-port correlated $(0.7 \lambda)$ cross-polarized antennas) will be used in the remainder of this thesis in order to minimize the number of antenna configurations and focus solely on the impairments which will be introduced in the following sections.

\subsection{Phase Noise Effects on the LTE Downlink}

LTE and other communication systems that up-convert baseband signals to higher frequencies for transmission are subject to noise which degrades the performance of the system. The effects of the noise can cause deterioration of the performance of the system if it is not properly compensated. This section looks at the deterioration caused by phase noise on the signal transmit from the antenna ports of a 4 transmitter base station.

In order to transmit the downlink to the UE, oscillators are used in radio communication systems to up-convert baseband signals to passband for transmission over the wireless channel. Noise in the system can cause the LO to wander and spread the signal peak in the frequency domain, reducing the purity of the $\mathrm{LO}$ as seen in figure 4.13. In the frequency domain, the random phase and frequency changes in the LO output are characterized by the amount of power measured at an offset (eg. $100 \mathrm{~Hz}, 1 \mathrm{kHz}, 10 \mathrm{kHz}, 100 \mathrm{kHz})$ from the carrier frequency, in $\mathrm{dBc}$, and is known as phase noise.

The frequency domain phase noise can be related to phase jitter in the time domain. Jitter is typically used in the digital context when clock sources, microprocessors and sampling circuits are employed. Jitter is a measure of the difference between the actual clock signal and an ideal reference clock or oscillator. Engineers use measurements such a cycleto-cycle jitter and absolute jitter to measure the quality of the oscillator used to generate the clock signal in order to determine how the sampled signal is affected. A common problem in sampled systems is a deterioration of the SNR of the signal as the jitter, sampling frequency and bandwidth of the input signal increase.

The purity of the oscillators in an LTE transmitter or receiver is of interest to designers since there is a need to trade-off oscillator quality, size, cost, power consumption and the 

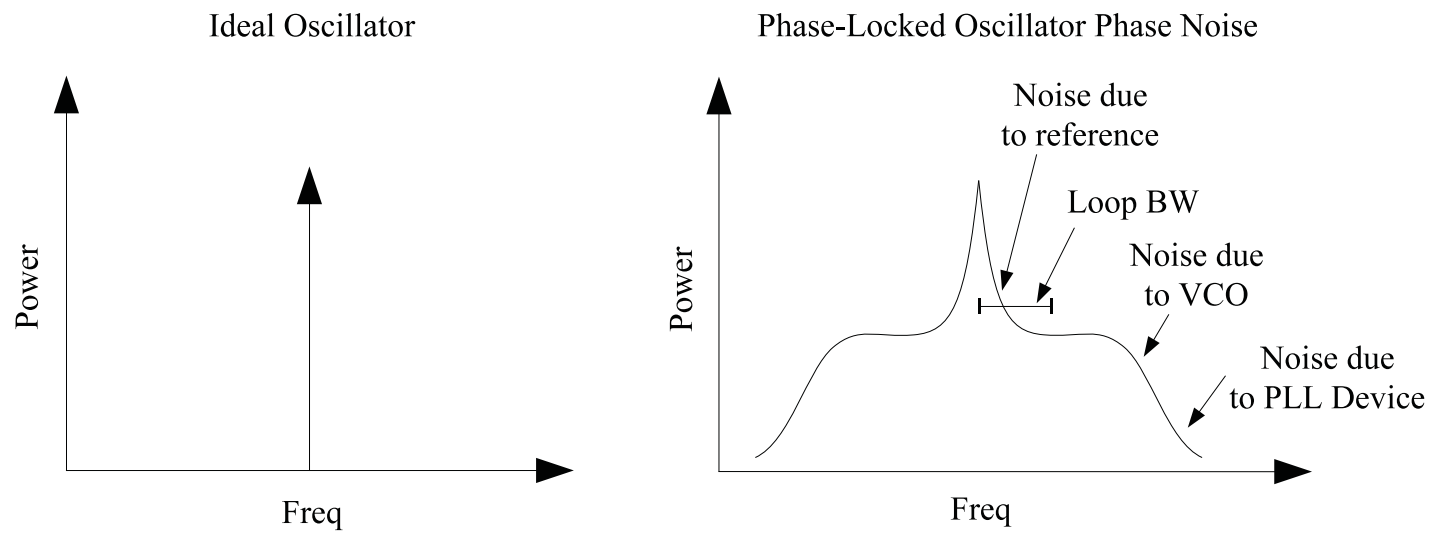

Figure 4.13: Ideal versus Practical Oscillator

quality of the LOs and references. In addition to these design constraints, noise in the system and thermal noise can modulate the output of the oscillators used to perform various functions such as baseband signal up-conversion and sampling or analog signal generation using analog to digital converters (ADCs) or digital to analog converters (DACs). Despite careful radio front end design, there are many possible noise sources in a radio product which can cause phase noise at the antenna ports.

Examples of possible noise sources in an LTE radio are:

- One or more reference signals and the random phase jitter between the references.

- The phase noise caused by the phase-locked loops (PLLs) and its supporting circuitry (voltage controlled oscillator (VCO), loop filter).

- Voltage regulators that provide power to the reference(s), PLLs and VCOs.

- Digital circuits that are not well isolated from the sensitive analog circuit of the LO.

- Other unaffiliated system circuits (eg. clocks, coils, etc) that may be interfering with or facilitating the coupling of noise into the LO signal.

In modern digital communication systems the waveforms used for transmission are becoming increasingly complex and require certain characteristics to be maintained, otherwise degradation can occur. In OFDM systems, orthogonality needs to be maintained between the sub-carriers. However, when sample clock jitter or RF LO phase noise is introduced into the transmit signals by non-ideal clocks or oscillators, common phase error (CPE) and adjacent carrier signal leakage can contaminate the adjacent sub-carriers. 
In this section, the effect of the noise in the system on the signals transmit from the antenna ports of a 4 transmitter base station is investigated. Section 4.2.1 discusses the phase noise model used, signal to interference plus noise ratio (SINR) and the effect on throughput. A reference simulation which uses generated phase noise is presented in section 4.2.2 to gain an understanding of the effects of different levels of RMS phase noise before injecting the lab measured noise. The simulation results that include the lab measured phase noise are then presented in section 4.2.3 where the total phase jitter is measured between 2 antenna ports and then injected into an LTE link level simulator to investigate the potential effects of phase noise on the downlink throughput.

\section{Phase Noise Effect on the BER and Throughput of an OFDM System}

In OFDM systems, the transmitted baseband time domain samples are calculated using equation 4.1. The modulated symbols $X_{k, l}{ }^{6}$ in equation 4.1 are converted to the time domain signal $y(t)$ via the inverse FFT before transmission. To model phase noise, a complex exponential term is added with the random phase variable $\theta(t)$.

$$
y(t)=\sum_{k=-N / 2}^{N / 2-1} X_{k} \cdot e^{j 2 \pi k \Delta f t / N} \cdot e^{j \theta(t)}
$$

At the receiver, the received symbol at the $m^{\text {th }}$ sub-carrier $Y_{m}$ is found by taking the FFT of the received time domain samples $y(n)$ as shown in equation 4.2.

$$
Y_{m}=\frac{1}{N} \sum_{n=-N / 2}^{N / 2-1} y(n) e^{-j 2 \pi m \Delta f n / N}
$$

The effect of the phase noise modeled by the complex exponential in equation 4.1 is accounted for by taking the FFT of equation 4.1 and defining the term $C(k)=$ $(1 / N) \sum_{n=-N / 2}^{N / 2-1} e^{(2 \pi \Delta f n k / N+\theta(t))}$ as shown in [38]. By grouping the terms into the desired received signal $Y_{m}$ and separating the unwanted effect caused by ICI (the adjacent sub-carrier signal leakage) the equation for the received symbol affected by phase noise is obtained in equation 4.3 .

$$
Z_{m}=\underbrace{Y_{m}}_{\text {Desired symbol }} \cdot \underbrace{C(0)}_{\text {Common phase error }}+\underbrace{\sum_{k=-N / 2, k \neq m}^{N / 2-1} X_{k} \cdot C(k-m)}_{\text {ICI from adjacent symbols } X_{k}}
$$

\footnotetext{
${ }^{6}$ In further analysis the sub-script $l$ selecting the current OFDM symbol is dropped for simplicity.
} 
The received signal affected by phase noise in equation 4.3 clearly shows the 2 effects cause by phase noise. The first, the constant phase error (CPE) is represented by the coefficient $C(0)$. Luckily, the common phase error coefficient $C(0)$ causes a phase rotation that is common to all sub-carriers since it is not dependent on the sub-carrier index. The CPE can be estimated using known reference signals as long as the changes in the coefficient are due to a slowly varying process.

On the other hand, the coefficient $C(k-m)$ can be viewed as unwanted leakage from adjacent transmit sub-carriers $X_{k}$, where $k \neq m$, into the received symbol $Z_{m}$. The leakage from the adjacent sub-carriers into the received symbol $Z_{m}$ is known as ICI and the amount of leakage is dependent on the coefficient $C(k-m)$ which in turn depends on the power spectrum of the LO.

ICI is problematic since it is difficult to mitigate against and the residual ICI causes error floors and throughput ceilings. The received symbol $Z_{m}$ contains signal power from the desired symbol $Y_{m}$ and the sum of the interference from the adjacent sub-carrier $X_{k}$ where $k \neq m$ multiplied by the factor $C(k-m)$. The signal-to-interference plus noise ratio (SINR) for a system with ICI is shown in equation 4.4 from [39].

$$
S I N R=\frac{E_{s}}{E_{s} \sigma_{I C I}^{2}+\sigma_{n}^{2}}
$$

In the noise limited region, where the thermal noise $\sigma_{n}$ dominates, the SINR is approximated by $E_{s} / \sigma_{n}$. The signal power can be increased and a similar increase in system performance will follow, ie. the bit error rate (BER) will decrease and higher throughput will be observed. However, the detrimental effect of ICI becomes apparent in the interference limited region where the SINR is dominated by $E_{s} /\left(E_{s} \sigma_{I C I}^{2}\right)$ and despite increases in the signal energy $E_{s}$ the SINR reaches the ceiling shown in equation 4.5.

$$
S I N R \approx \frac{1}{\sigma_{I C I}^{2}}
$$

The approximate SINR limit shown in equation 4.5 results in an upper limit on the throughput and an error floor in the interference limited region, as shown in figure 4.14.

\subsubsection{Phase Noise Model}

The generated and lab measured phase noise was injected into the baseband signal using the complex exponential term seen in equation 4.1. 

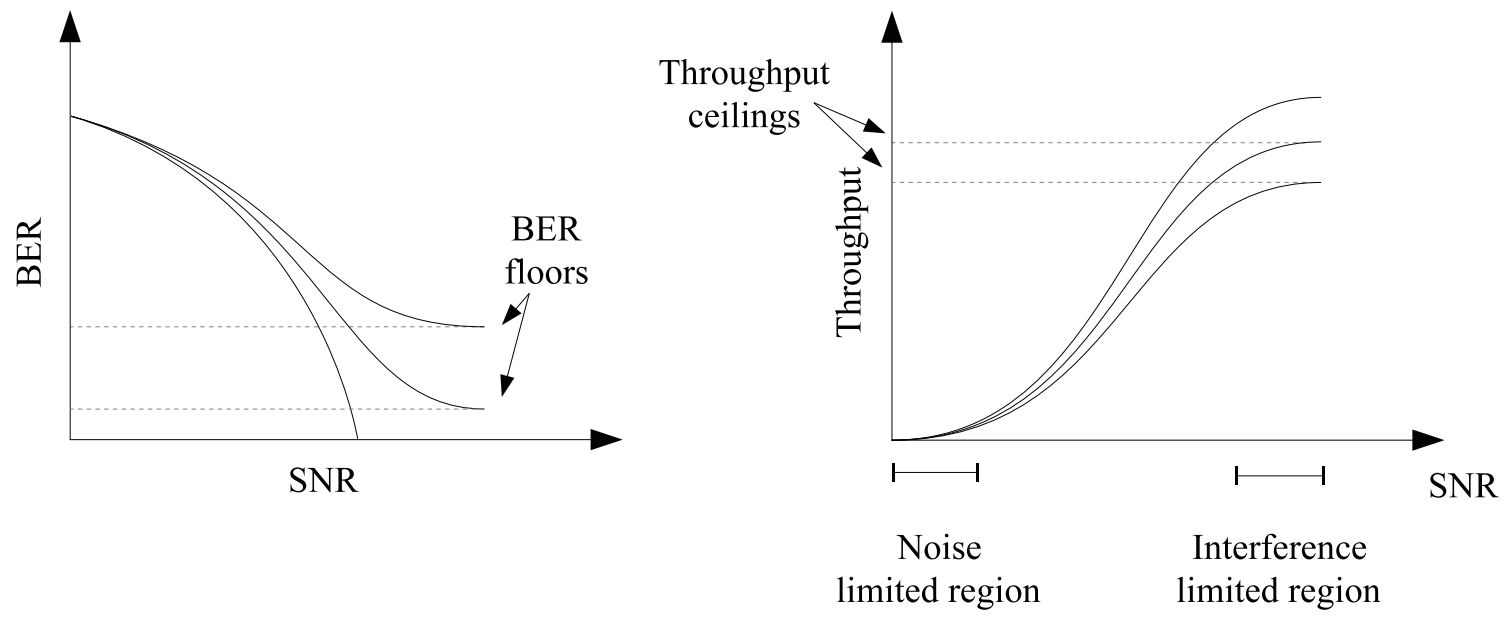

Figure 4.14: Error Floors and Throughput Ceilings due to ICI

The variable $\theta(t)$, shown in equation 4.1, is a random variable that is used to model the phase noise spectrum of an oscillator. The Lorentzian spectrum, seen in equation 4.6, is a popular model which approximates the phase noise of a phase-locked oscillator circuit. A characteristic of the Lorentzian spectrum is that it broadens the signal peak but maintains the total power of the original signal across $[-\infty, \infty] \mathrm{Hz}$ frequency range. The characteristic broadening of the signal is important to model since it induces ICI in OFDM systems.

$$
L(f)=\frac{2}{\pi \Delta f_{3 d B}} \cdot \frac{1}{1+\left[2 f /\left(\Delta f_{3 d B}\right)\right]^{2}}
$$

Another method used to approximate the phase noise of an oscillator which is simpler than using the Lorenztian model is to inject filtered white Gaussian noise into $\theta(t)$ of the complex exponential term of equation 4.1. The filtered noise method uses a low pass filter of the desired bandwidth to approximate the phase noise spectrum of an LO. The filtered noise is then applied to the transmitted baseband signal using the random phase variable $\theta(t)$.

In section 4.2.2, the phase noise is modeled in the simulator using the filtered white Gaussian noise method. The noise is filtered with a low pass filter (LPF) with a $10 \mathrm{kHz}$ bandwidth to approximate the phase noise spectrum of an LO. The filtered noise data is then used to add phase noise to the time domain signal after IFFT and CP addition by multiplying the time domain samples by the complex exponential term $e^{j \theta(t)}$ just before transmitting the signal across the channel.

The purpose of the simulations performed in section 4.2.2, which use generated phase noise and EPA channel model, is to evaluate the effect of phase noise present at the BS 
antenna ports on the throughput of the LTE downlink. Three throughput curves are plotted. The first curve is the downlink throughput of an LTE system without phase noise. The second and third throughput curves are impaired with generated phase noise with standard deviations of $4^{\circ}$ and $8^{\circ}$ RMS. The curves are also used as a reference to better understand the level of degradation that could potentially occur in a system impaired by real phase noise, as modeled in section 4.2.3.

The phase noise used in the simulations in section 4.2.3 was obtained from measurements taken on actual radio equipment. The practical noise measurements contain the sum of the effects of the noise sources present in the radio, including periodic phase shifts. The holistic phase noise measurements will help understand the throughput degradation caused by all noise sources in an actual production radio unit.

In both cases the first antenna port was used as the reference antenna port and no phase noise was applied to the first port. The phase noise was injected into the remaining ports as described in the introduction to the generated phase noise simulations and the measured phase noise simulations in sections 4.2.2 and 4.2.3 respectively.

Finally, it is important to note that in all simulations, the throughput degradation is due to the residual effect of the phase noise since the LTE link level simulator implements similar algorithms used in a base station and UE to decode the downlink. The algorithms use knowledge of the LTE downlink sub-frame reference signals to estimate and attempt to correct for the effects introduced by the phase noise. The simulations will show how the throughput is affected by the residual CPE and the ICI caused by the base station transmitter chain and RF section.

\subsubsection{Reference Simulations using Generated Phase Noise and the 3GPP EPA Channel Model}

In this section, reference phase noise simulations are run using the 3GPP testing and conformance channel model, known as EPA in order to determine the effect of the residual phase noise on the downlink sub-carriers at the receiver. The reference phase noise simulations use generated phase noise with standard deviations of 0 (no phase noise), 4 and 8 degrees RMS and bandwidth of $10 \mathrm{kHz}$. The noise is injected into the time domain signal after IFFT and CP addition using the complex exponential containing the time varying phase term $\theta(t)$ as shown in equation 4.1 and as discussed in section 4.2.1. Individually generated phase noise is applied to antenna ports $\{1,2,3\}$ and the first antenna port, port 0 is used as the reference port therefore no noise is applied to it. 
The reference phase noise simulations using the EPA channel model are performed with similar system settings to the measured phase noise simulations executed in section 4.2.3 in order to be able to compare the results. The parameters for the reference simulation shown in table 4.13 are similar to the parameters in table 4.15 except for the antenna geometry and channel model required for the SCME simulations.

Two velocities were simulated to show the effect of the throughput at $3 \mathrm{~km} / \mathrm{hr}$ and 30 $\mathrm{km} / \mathrm{hr}$. For additional simulation details, the reader is referred to table 4.13 .

\section{Generated Phase Noise Simulation Results}

The EPA generated phase noise simulation results presented in figure 4.15a, $4.15 \mathrm{~b}$ show the effect of phase noise at low velocity. Three curves are plotted for each transmission mode where the first curve is the ideal curve and the other two curves represent the throughput using $4^{\circ}$ and $8^{\circ}$ RMS phase noise. As expected, an increase in phase noise reduces the throughput. In the case of TM4 rank-1, it appears that the phase noise with a standard deviation of $4^{\circ}$ RMS has only a small effect. The measured loss of TM4 rank-1 at 4 Mbps is approximately $0.5 \mathrm{~dB}$ and $0.6 \mathrm{~dB}$ for the EPA simulation with high antenna correlation and low antenna correlation, respectively. The same transmission mode has a loss of 1.9 $\mathrm{dB}$ SNR and 3.4 dB SNR when the standard deviation of the phase noise is increased to $8^{\circ}$ RMS.

When looking at the effect of the phase noise across the SNR range the phase noise has noticeably less throughput degradation effect at low SNR than at high SNR. At low SNR the dominating noise term of the signal-to-interference plus noise ratio (SINR), in equation 4.4, is the thermal noise. In the noise limited region an increase in signal power, or SNR, corresponds to increasing throughput as expected. On the other hand, an increase in transmit signal power in the interference limited region (beyond $10 \mathrm{~dB}$ of SNR) does not correspond to the same increase in throughput, and gradually the throughput ceiling is reached. This is because in the interference limited region the dominating term in the SINR equation 4.4 is the ICI term which imposes and upper limit on the throughput based on the amount of ICI present.

Figures $4.16 \mathrm{a}$ and $4.16 \mathrm{~b}$ represent the throughput affected by phase noise at $30 \mathrm{~km} / \mathrm{hr}$. As in the low velocity simulation, TM4 rank-1 is less sensitive to the generated LO phase noise than transmit diversity for the EPA simulation using high antenna correlation. Closedloop spatial multiplexing only exhibits a small amount of degradation at high SNR with a 
Table 4.13: Simulation Parameters for the Generated Phase Noise Simulations

\begin{tabular}{|c|c|c|}
\hline Category & Parameter & Value \\
\hline \multirow{5}{*}{ System } & Bandwidth@Freq & $20 \mathrm{MHz}$ (100 PRBs)@2.6 GHz \\
\hline & Duplex mode & FDD \\
\hline & Sub-carriers & $15 \mathrm{kHz}$ \\
\hline & Cyclic prefix & Normal CP $(4.7 \mu \mathrm{sec})$ \\
\hline & Simulation duration & 15,000 sub-frames \\
\hline \multirow{2}{*}{ BS antennas } & Configurations & fig $4.1 \mathrm{c}, 4.1 \mathrm{~d}, 4.2 \mathrm{~b}, 4.2 \mathrm{c}^{\mathrm{a}}$ \\
\hline & Correlation & Low and high \\
\hline \multirow{3}{*}{ Channel } & Channel model & EPA \\
\hline & UE velocity & $3,30 \mathrm{~km} / \mathrm{hr}$ \\
\hline & SNR & Swept ( -10 to $30 \mathrm{~dB})$ \\
\hline \multirow{3}{*}{ Resources/Scheduling } & Transmission modes & TM2 and TM4 rank-1 \\
\hline & UE resource blocks & $8 \mathrm{PRB}$ \\
\hline & OLLA & On \\
\hline \multirow{7}{*}{ Measurements/Reporting } & Channel estimation & MMSE \\
\hline & RS power boosting & $6 \mathrm{~dB}$ \\
\hline & CQI reporting interval & $5 \mathrm{msec}$ \\
\hline & CQI processing delay & $(\mathrm{UE}+\mathrm{BS}) 8 \mathrm{msec}$ \\
\hline & CQI sub-band group size & 8 PRB \\
\hline & PMI group size & 8 PRB \\
\hline & HARQ & 3 re-transmissions, 8 processes \\
\hline
\end{tabular}

${ }^{\text {a }}$ EPA uses antenna correlation matrices instead of antenna geometry (eg. spacing). 


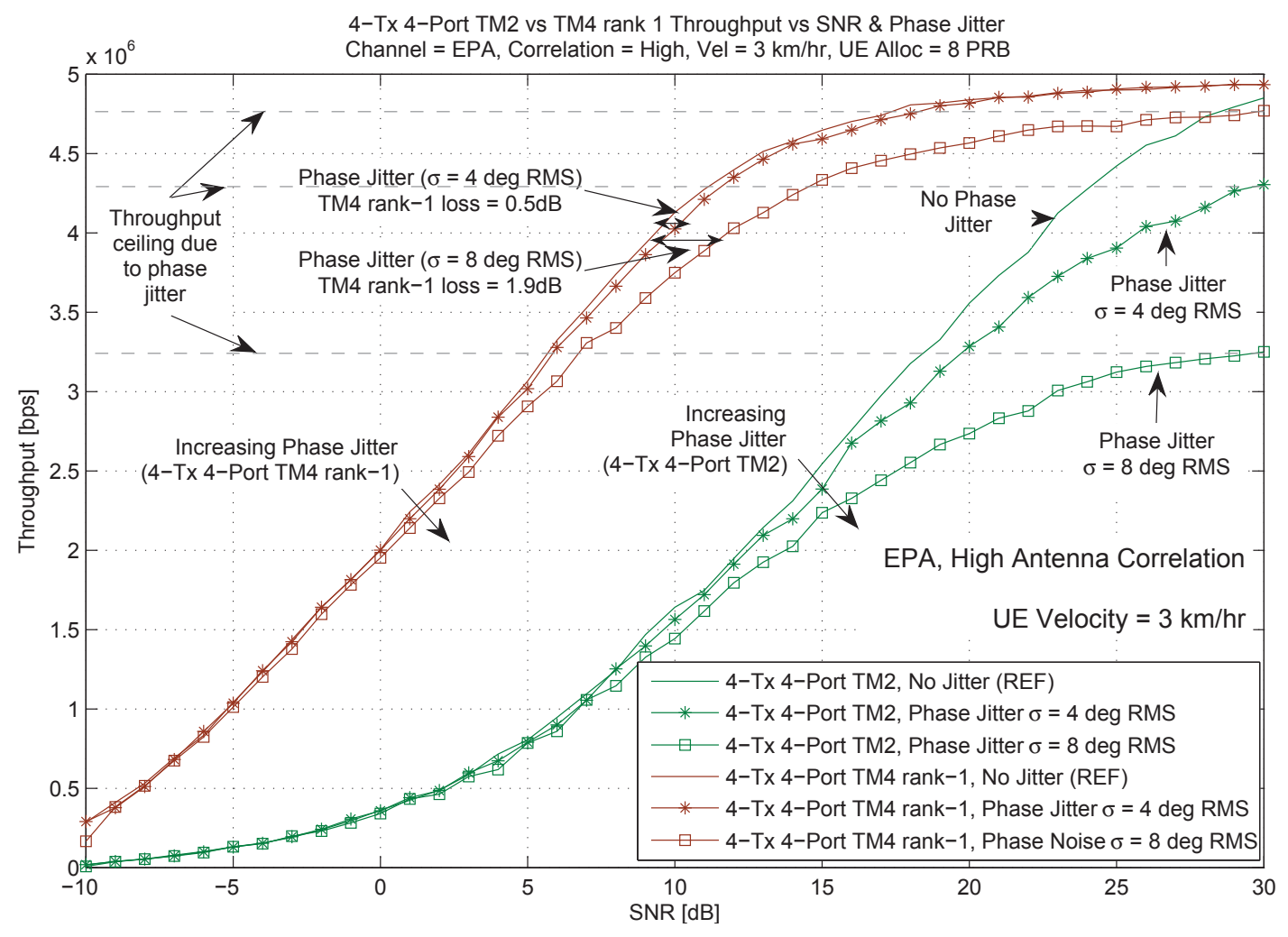

(a) EPA, High Antenna Correlation, UE Velocity $3 \mathrm{~km} / \mathrm{hr}$

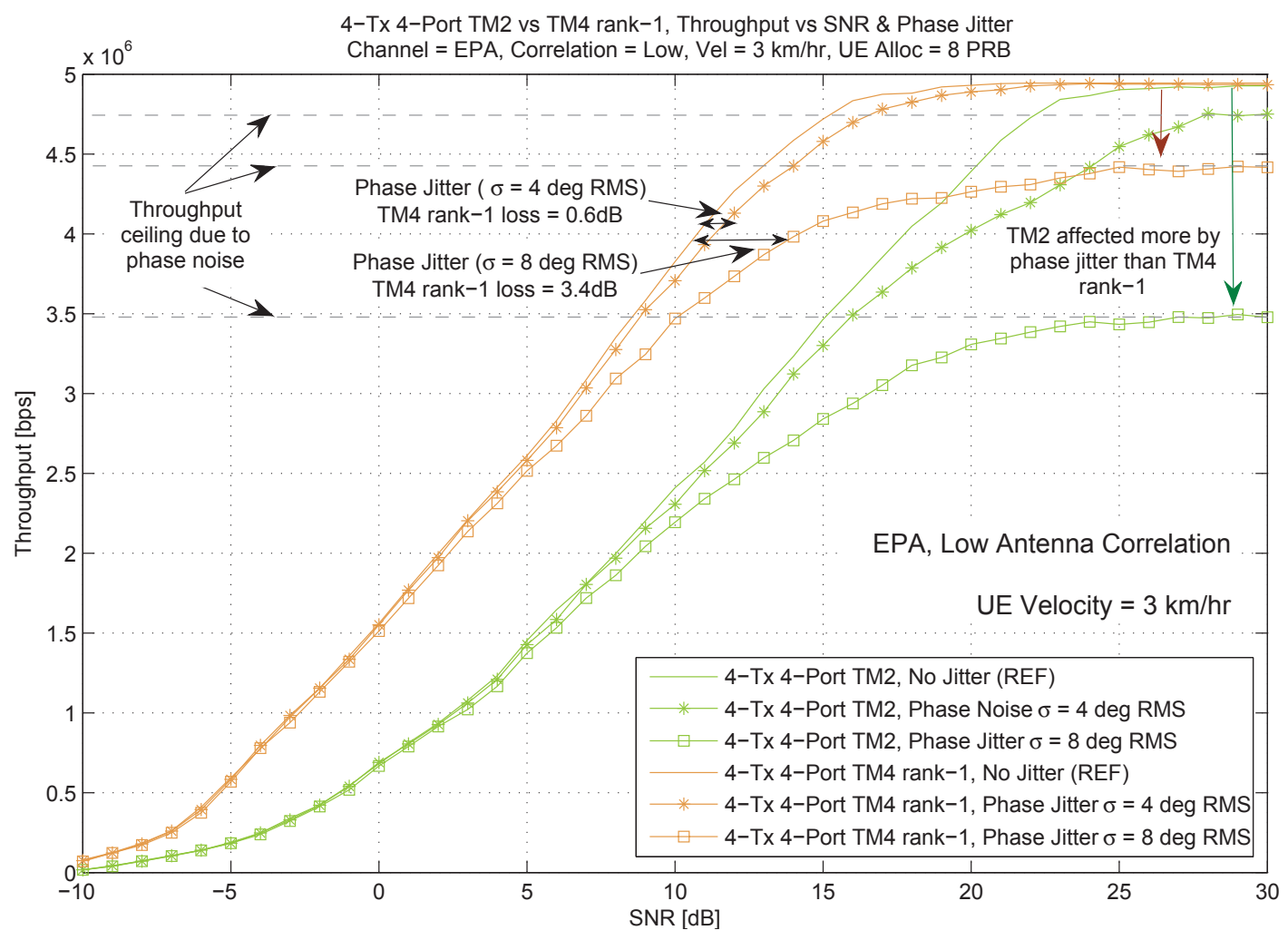

(b) EPA, Low Antenna Correlation, UE Velocity $3 \mathrm{~km} / \mathrm{hr}$

Figure 4.15: Phase Noise Effect on the LTE Downlink using TM2 and TM4 rank-1 and the EPA Channel Model with High and Low Antenna Correlation at $3 \mathrm{~km} / \mathrm{hr}$ 
phase noise standard deviation of $4^{\circ} \mathrm{RMS}$ while on the other hand the degradation is similar for both TM2 and TM4 rank-1 when low antenna correlation is used.

In summary, the throughput degradation observed in the EPA simulations for transmit diversity is more pronounced than when using closed-loop spatial multiplexing. The ICI affects the channel estimates which transmit diversity relies heavily on and are not assumed to change significantly over space, frequency and time in SFBC-FSTD. This fact along with the higher throughput performance of spatial multiplexing in most cases supports the use of spatial multiplexing over the use of transmit diversity even if transmit diversity is established as the fall-back mode in LTE.

In the case of closed-loop spatial multiplexing, TM4 rank-1 is more resilient to phase noise than transmit diversity, in 3 out of 4 cases presented and only exhibits a small $0.6 \mathrm{~dB}$ of degradation at low velocity at $4 \mathrm{Mbps}$. In a practical system, the designer should attempt to minimize the phase noise but a phase noise standard deviation of up to $4^{\circ}$ RMS does not cause a detrimental decrease in throughput when using closed-loop spatial multiplexing with the preferred high antenna correlation setup even at $30 \mathrm{~km} / \mathrm{hr}$.

In section 4.2.3, phase noise data gathered from an actual LTE radio will be used to simulate phase noise impairments on the LTE downlink. The analysis will show that the sampled phase noise has a low standard deviation compared to the $4^{\circ}$ RMS phase noise used here. Based on the results using generated phase noise in this section, the sampled phase noise data sets used in section 4.2.3 are not expected to affect the system performance significantly.

\subsubsection{Measured Phase Noise Simulations}

In this section, sampled phase noise taken from measurements made on an actual base station radio is injected into the simulator to help determine if any downlink throughput loss occurs due to real phase noise within a base station. In this section, the phase noise is applied to the time domain samples before transmission using the same complex exponential multiplication as described in section 4.2.1 with real phase noise data sets taken from lab measurements described in section 4.2.3. 


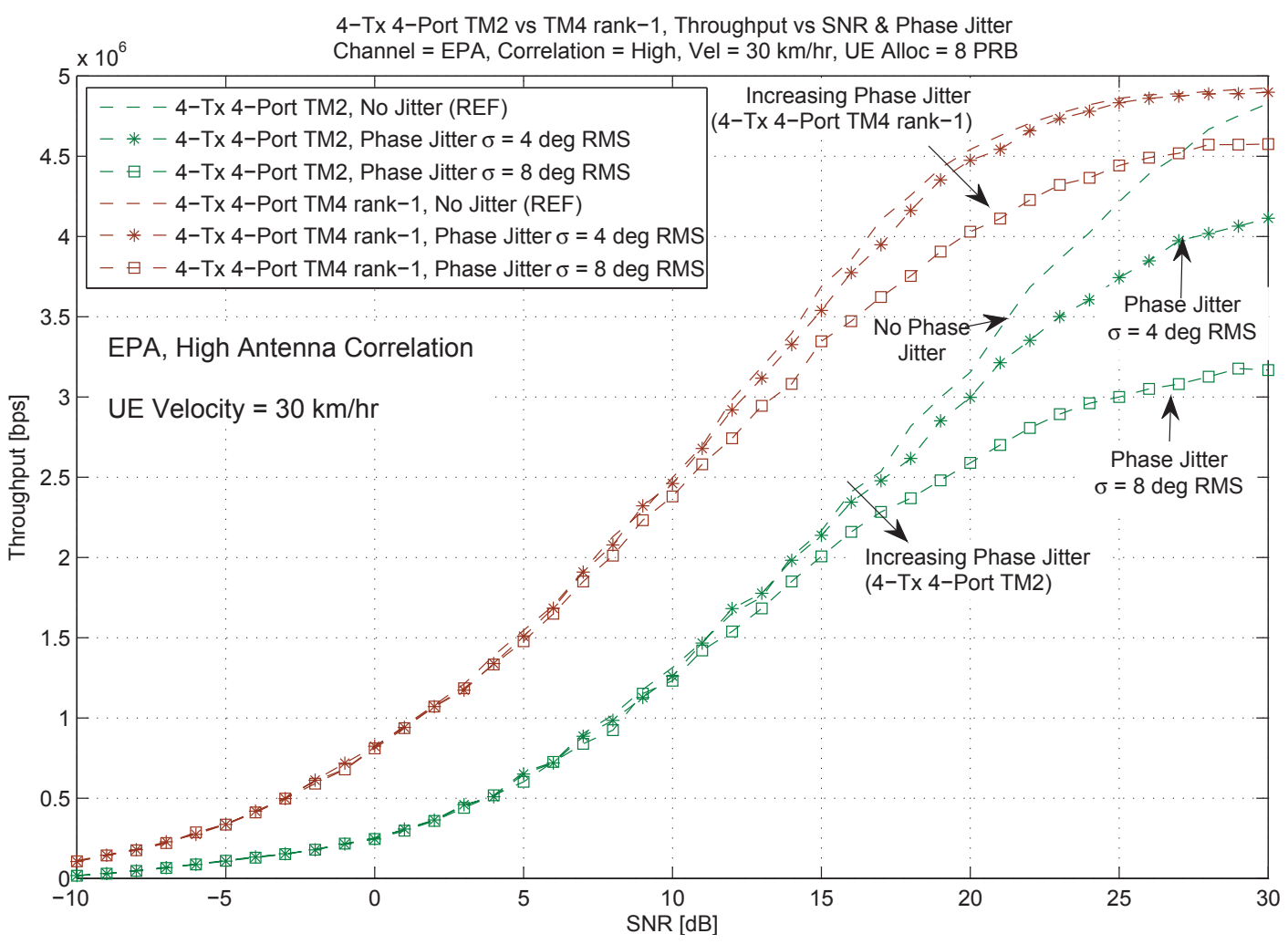

(a) EPA, High Antenna Correlation, UE Velocity $30 \mathrm{~km} / \mathrm{hr}$

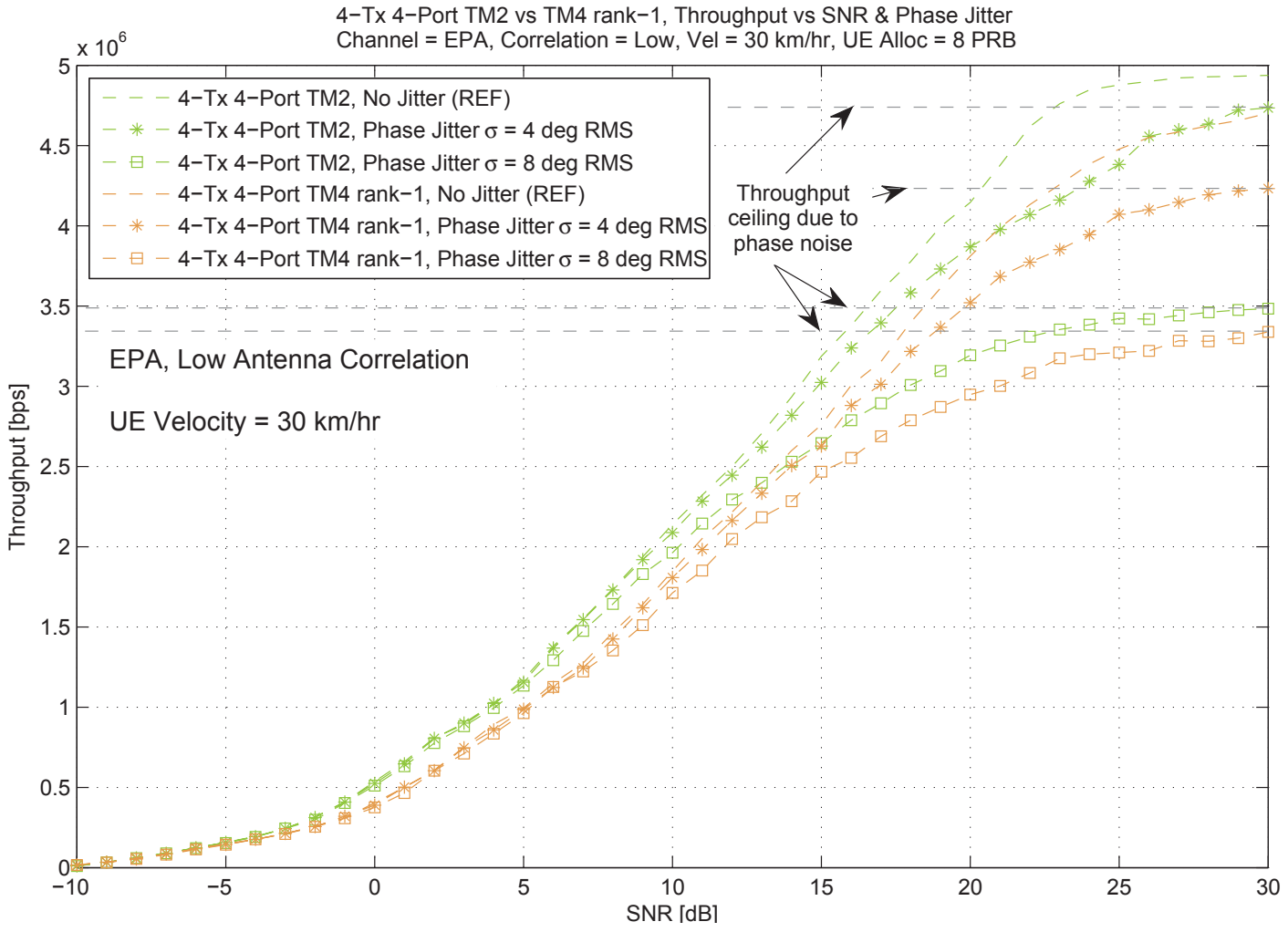

(b) EPA, Low Antenna Correlation, UE Velocity $30 \mathrm{~km} / \mathrm{hr}$

Figure 4.16: Phase Noise Effect on the LTE Downlink using TM2 and TM4 rank-1 and the EPA Channel Model with High and Low Antenna Correlation at $30 \mathrm{~km} / \mathrm{hr}$ 


\section{Phase Noise Measurement Model}

Figure 4.17 represent the radio configuration for a 4 port BS setup used for measurements. The 4 port configuration with 2 radios was used to take the phase noise data set measurements as well as to run the simulations to evaluate the throughput degradation with the measured data sets. Each radio has two output RF ports that are locked to a reference signal provided by the common public radio interface (CPRI).

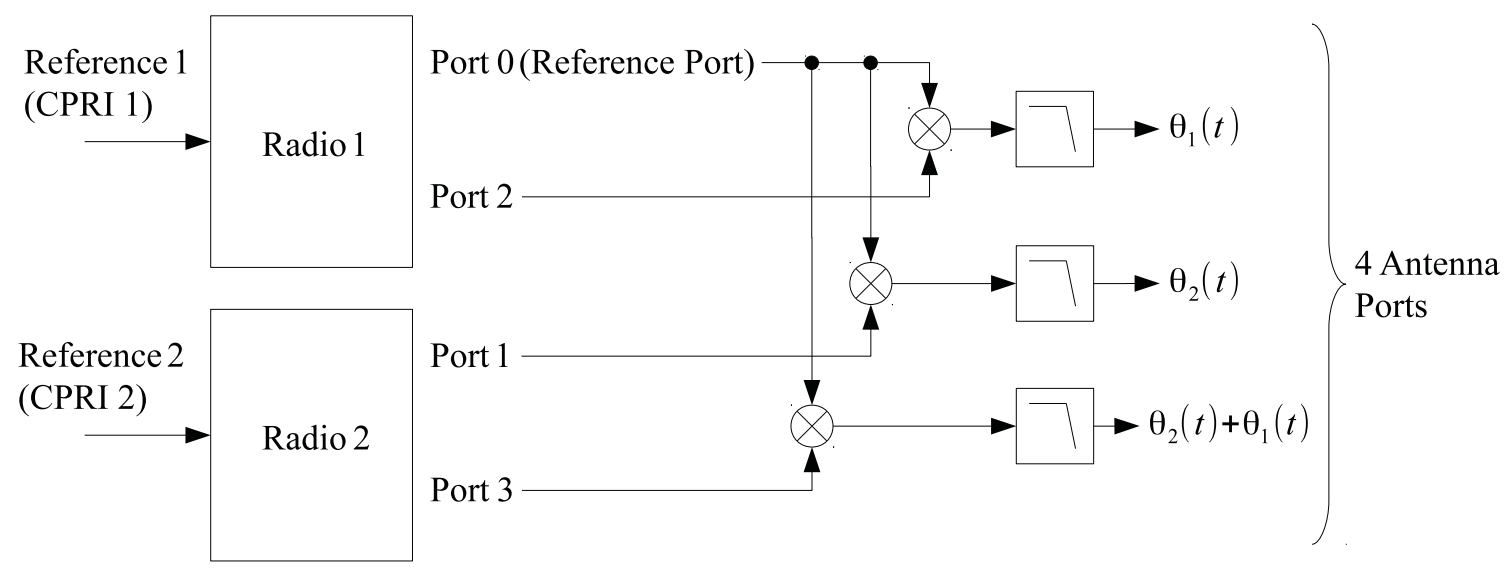

Figure 4.17: Phase Noise Measurement Between Ports

In order to gather the phase noise data sets, the radios are configured to output sine waves at the antenna ports in a predefined system test mode. The measured phase noise data sets, $\theta_{1}(t)$ and $\theta_{2}(t)$, are always taken with reference to antenna port 0 , the reference antenna port, using a mixing operation. Phase alignment, amplitude adjustment are used to calibrate the sine waves and then low pass filtering, shown in figure 4.17 is used to isolate the phase noise difference between the two ports. The mixing operation before low pass filtering is equivalent to mixing two sinusoids with amplitudes $A_{1}, A_{2}$, phase offset $\theta_{\text {offset }}$ and time variant phase noise term $\theta_{\text {jitter }}(t)$ shown in equation 4.7 .

$\left.A_{1} \sin \left(\omega_{c} t\right) A_{2} \sin \left(\omega_{c} t+\theta_{\text {offset }}+\theta_{\text {jitter }}(t)\right)=\frac{A_{1} A_{2}}{2}\left[\theta_{\text {offset }}+\theta_{\text {jitter }}(t)\right)-\cos \left(2 \omega_{c} t+\theta_{\text {offset }}+\theta_{\text {jitter }}(t)\right)\right]$

When the test sinusoids are carefully calibrated, the amplitudes $A_{1}$ and $A_{2}$ are matched as closely as possible during measurements and therefore the amplitudes can be considered approximately equivalent $A_{1}=A_{2}=A$. In addition, the constant phase offset $\theta_{\text {offset }}$ was nulled by adjusting the phase of one of the test sinusoids. In equation 4.8 two cosine 
terms remain, therefore low pass filtering was used to isolate only the low frequency term, leaving just the amplitude multiplied by the cosine of the time variant phase jitter $\theta_{\text {jitter }}(t)$. The amplitude data can be collected by using an oscilloscope and the amplitude at time $t$ can be used to extract the phase jitter $\theta_{\text {jitter }}(t)$ from the amplitude data with Matlab, during offline processing.

$$
A \sin \left(\omega_{c} t\right) A \sin \left(\omega_{c} t+\theta_{j i t t e r}(t)\right)=\frac{A^{2}}{2}[\underbrace{\cos \left(\theta_{\text {jitter }}(t)\right)}_{\text {Phase Jitter }}+\underbrace{\cos \left(2 \omega_{c} t+\theta_{j i t t e r}(t)\right)}_{\text {Removed after low pass filtering }}]
$$

\section{Measured Phase Noise Data Sets}

Two measured phase noise data sets are used to model the 4 antenna base station since each radio unit only provides 2 output antenna ports as shown in the system model in figures 4.17 and 4.22. The two phase jitter measurements were taken as described in section 4.2.3 and labeled $\theta_{1}(t)$ and $\theta_{2}(t)$.

The first data set labeled $\theta_{1}(t)$ in figure 4.17 is the instantaneous phase difference between the reference port Port 0 and the remaining port Port 2 on the same radio. The phase noise data set $\theta_{1}(t)$ between two ports on the same radio is relatively quiet, as expected, since the reference for both output ports is from the same CPRI. Noise common to both ports, known as common mode noise in electronics, would not cause phase differences between two ports with the same noise source since the mixing operation done during measurement would reject this common mode noise.

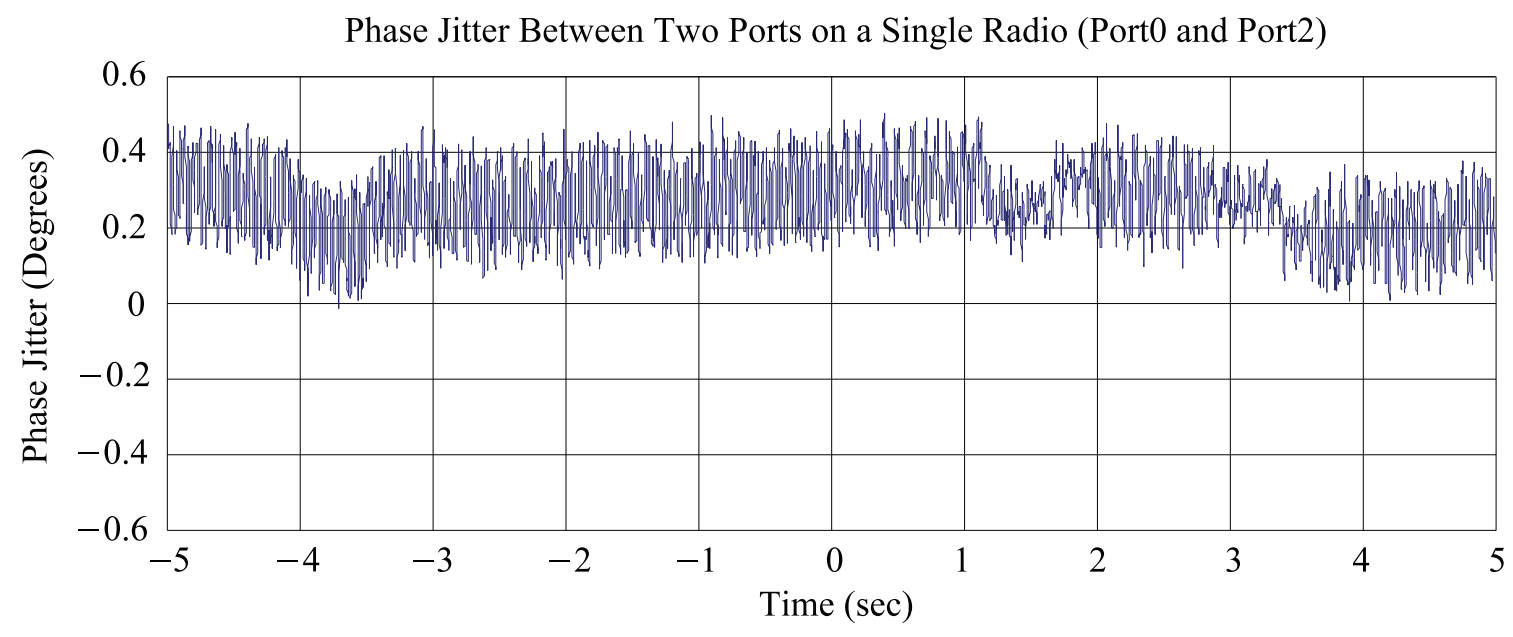

Figure 4.18: Phase Noise Data Set $\theta_{1}(t)$ 


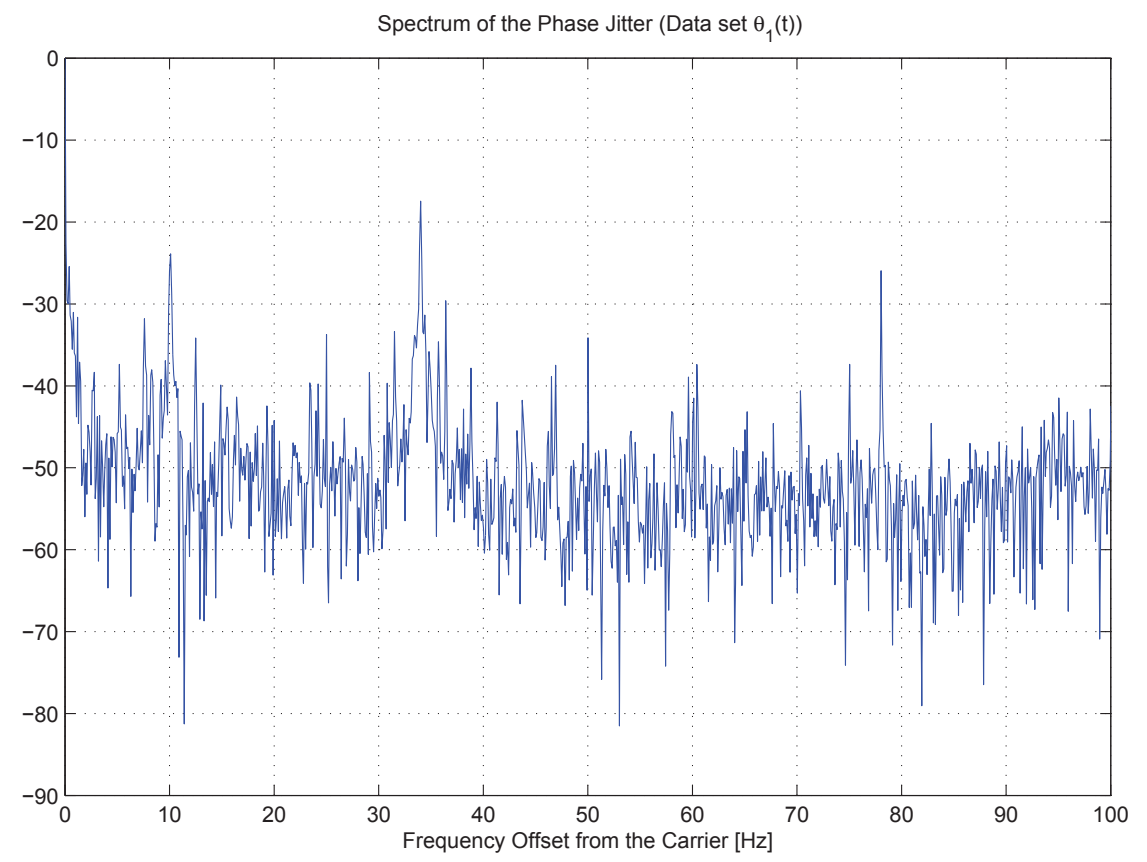

Figure 4.19: Spectrum of Phase Noise Data Set $\theta_{1}(t)$

The spectrum of phase jitter data set $\theta_{1}(t)$ and data set $\theta_{2}(t)$ are given in figures 4.19 and 4.21 to show that the random phase jitter data sets appear to be relatively slowly changing random processes. In both figures, the significant components are within a small bandwidth which implies a slowly changing common phase error (CPE) process in the time domain which should be mostly correctable by the downlink estimation and correction algorithms. The throughput degradation will be due to the remaining higher frequency components of the CPE and the ICI.

The second data set $\theta_{2}(t)$ is the measured instantaneous phase difference between the reference port Port 0 and the first port of the second radio, labeled Port 1 . The second set is used to model the phase jitter between the radios. Both Port 1 and Port 3 on the second radio use data set $\theta_{2}(t)$ to model the phase jitter between the radios. In addition to data set $\theta_{2}(t)$, Port 3 also adds data set $\theta_{1}(t)$ in order to model the jitter between radios and Port 1 .

In the second data set, $\theta_{2}(t)$, a $7^{\circ}$ shift occurs at approximately a $2 \mathrm{~Hz}$ frequency. While the phase shift appears to be significant in amplitude the phase shift does not occur frequently and would only affect a few sub-frames on two occasions every 1000 sub-frames, or $1 \%$ of sub-frames. The receivers ability to measure and track the changing mean phase, $\mu_{\theta(t)}$, will determine the amount of throughput loss. The phase jitter would also cause some 


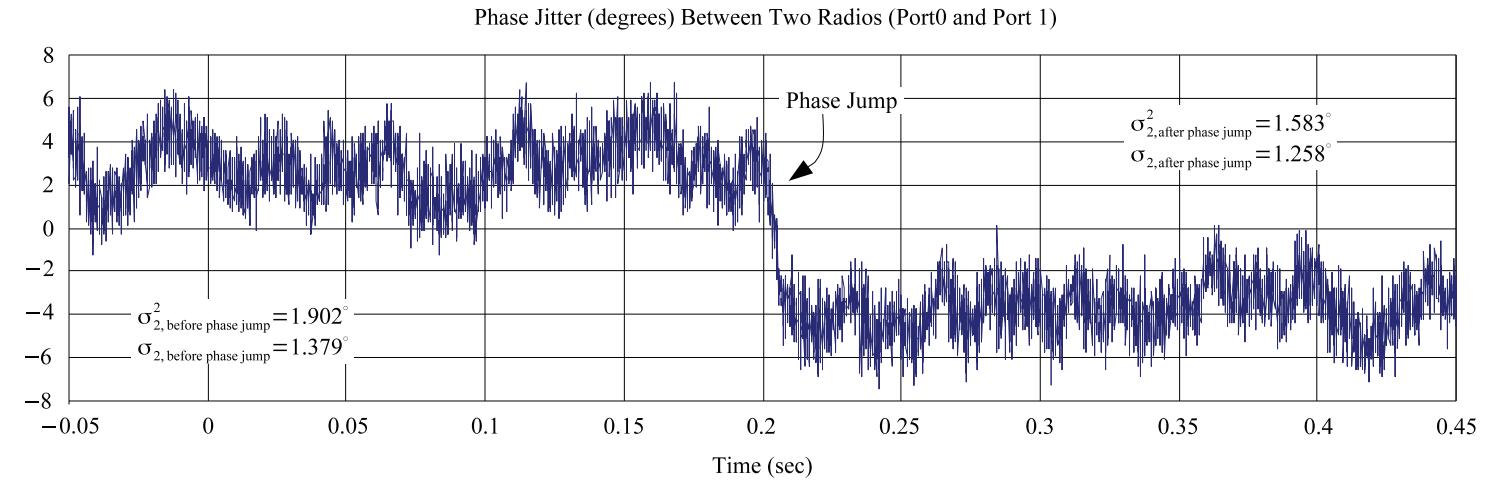

Figure 4.20: Phase Jitter Data Set $\theta_{2}(t)$

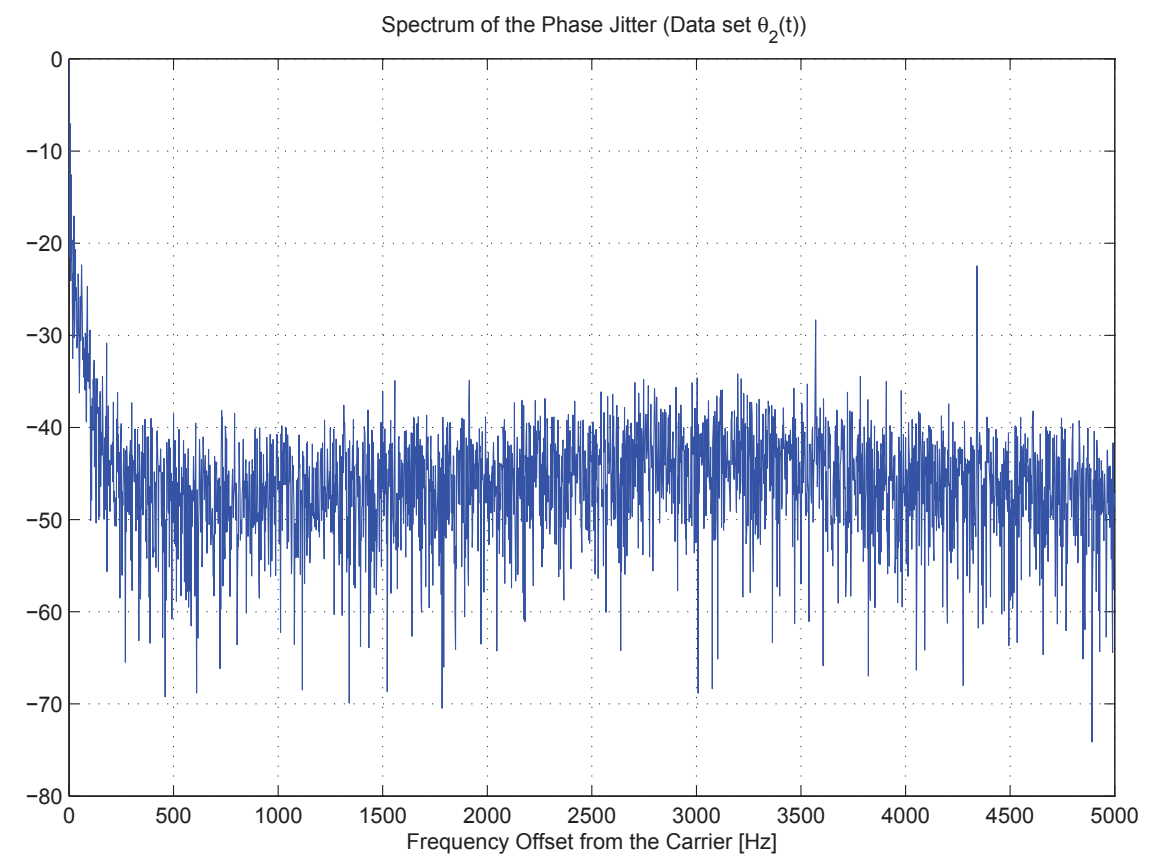

Figure 4.21: Spectrum of Phase Noise Data Set $\theta_{2}(t)$ 
amount of dithering to occur when using beamforming capable modes since the phases of the signals emit from the elements in the antenna array will not be ideal due to the phase jitter. The dithering could be beneficial if it is thought of as a form of diversity, similar to CDD used in TM3 and could help if the UE measurements that are used to generate the beams are not reliable due to measurement error.

The statistics for the phase jitter data sets shown in figures 4.18 and 4.20 are given in table 4.14.

Table 4.14: Phase Jitter Data Set Statistics

\begin{tabular}{lccc|cc}
\hline \multicolumn{1}{c}{ Data Set } & Variance & Std Dev & Mean Jitter & \multicolumn{2}{c}{ RMS Phase Jitter } \\
& & & & $\begin{array}{c}\text { RC+DC }) \\
\text { (AC Only) }\end{array}$ \\
& $\sigma^{2}$ & $\sigma$ & $\mu_{\theta(t)}$ & $\sqrt{\mu_{\theta(t)}^{2}+\sigma^{2}}$ & $\sigma$ \\
\hline \hline$\theta_{1}(t)$ & $0.010^{\circ}$ & $0.101^{\circ}$ & $0.263^{\circ}$ & $0.281^{\circ}$ & $0.101^{\circ}$ \\
$\theta_{2}(t)$ & $12.17^{\circ}$ & $3.490^{\circ}$ & $-0.383^{\circ}$ & $12.176^{\circ}$ & $3.490^{\circ}$ \\
$\theta_{2, \text { BeforePhaseJump }}(t)$ & $1.902^{\circ}$ & $1.379^{\circ}$ & & & $1.379^{\circ}$ \\
$\theta_{2, \text { AfterPhaseJump }}(t)$ & $1.583^{\circ}$ & $1.258^{\circ}$ & & & $1.258^{\circ}$ \\
\hline
\end{tabular}

Based on the RMS phase jitter of the two data sets the expected degradation would more likely be due to the second data set since the RMS phase jitter is multiple times higher than that of the phase jitter of the first data set which has an RMS jitter of $0.101^{\circ}$. The measured RMS jitter of the second data set is high if the observation period is across the whole set including the $7^{\circ}$ phase shift. However, it is possible to make observations about the portion of the set that occurs before and after the phase shift due to the in-frequent occurrence of the $7^{\circ}$ phase shift. Receivers are expected to be able to track and correct constant phase error on the ports caused by the low frequency phase differences between transmitters (eg. mean phase difference $\mu_{\theta(t)}$ ) by estimating the phase rotation based on the received reference symbols on each port. On the other hand, the higher frequency phase jitter causes ICI due to sub-carrier spreading into neighboring sub-carriers. The AC-only RMS phase jitter before and after the phase shift is a good indicator of the effect of the phase jitter. In the case of the second data set the RMS phase jitter observed is $1.902^{\circ}$ in the period before the phase shift and $1.583^{\circ}$ after the phase shift. In both cases, the degradation is expected to be minimal based on the throughput degradation caused by phase noise with a standard deviation of $4^{\circ}$ 
RMS observed in section 4.2.2.

\section{Real Phase Noise SCME Simulation Parameters}

Transmit diversity (TM2) and closed-loop spatial multiplexing (TM4 rank-1) were again used to evaluate the effect of real phase noise in this section. The duplexing mode, bandwidth, and other relevant settings are given in table 4.15. The antenna configuration is 4 transmitters and 2 receiving antennas and the effect of phase noise will be shown for mobile velocities of 3 and $120 \mathrm{~km} / \mathrm{hr}$ using the SCME channel with the urban macro environment selected.

A single BS-UE is simulated and the UE position is held constant at a distance of 550 meters away from the BS along the $\mathrm{x}$-axis (antenna array bore sight). The BS is considered the origin and the BS antenna elements are positioned at a height of 30 meters AGL.

The antenna port connections, element polarizations and the application of the phase jitter to the antennas to model phase noise are all important since they can potentially have an impact on the throughput degradation. Both polarizations are simulated and for each polarization, the antenna port connection and application of the phase jitter was chosen to give the maximum effect (worst case scenario analysis). For instance, in the simulations using the cross-polarized antennas, the phase noise data set with the largest standard deviation was used between the co-polarized element pairs, element $\{0,1\}$ and $\{2,3\}$, since these element pairs form beams and carry similar data. The phase noise between the cross-polarized pairs could cause less than optimal beamforming and difficulty at the decoder due to ICI. The BS antenna connections are shown in figure 4.22.

\section{Discussion of the SCME Simulation Results using Real Phase Noise}

The simulations results plotted in figure 4.23 and 4.24 are for both cross-polarized and co-polarized antennas at $0.7 \lambda$ and $10 \lambda$ element spacing.

The throughput degradation due to real phase noise injected into baseband time domain in the low UE velocity $(3 \mathrm{~km} / \mathrm{hr})$ curves is small. The throughput curves with phase noise do not deviate much from the reference ideal TM2 and TM4 rank-1 curves below $20 \mathrm{~dB}$ SNR. Where there is a reduction in throughput, the degradation is at most $0.45 \mathrm{~dB}$ and the loss is situated above $20 \mathrm{~dB}$ SNR. 
Table 4.15: Simulation Parameters for the Measured Phase Noise Simulations

\begin{tabular}{|c|c|c|}
\hline Category & Parameter & Value \\
\hline \multirow{5}{*}{ System } & Bandwidth@Freq & $20 \mathrm{MHz}$ (100 PRBs)@2.6 GHz \\
\hline & Duplex mode & FDD \\
\hline & Sub-carriers & $15 \mathrm{kHz}$ \\
\hline & Cyclic prefix & Normal CP $(4.7 \mu \mathrm{sec})$ \\
\hline & Simulation duration & 15,000 sub-frames \\
\hline \multirow{2}{*}{ BS antennas } & Configurations & fig $4.1 \mathrm{c}, 4.1 \mathrm{~d}, 4.2 \mathrm{~b}, 4.2 \mathrm{c}$ \\
\hline & Spacing & $0.7 \lambda$ and $10 \lambda$ \\
\hline \multirow{4}{*}{ Channel } & Channel model & SCME, Urban macro \\
\hline & Angular spreading & Low \\
\hline & UE velocity & $3,120 \mathrm{~km} / \mathrm{hr}$ \\
\hline & SNR & Swept (-10 to $30 \mathrm{~dB})$ \\
\hline \multirow{3}{*}{ Resources/Scheduling } & Transmission modes & TM2, TM4 rank-1 \\
\hline & UE resource blocks & $8 \mathrm{PRB}$ \\
\hline & OLLA & On \\
\hline \multirow{7}{*}{ Measurements/Reporting } & Channel estimation & MMSE \\
\hline & RS power boosting & $6 \mathrm{~dB}$ \\
\hline & CQI reporting interval & $5 \mathrm{msec}$ \\
\hline & CQI processing delay & $(\mathrm{UE}+\mathrm{BS}) 8 \mathrm{msec}$ \\
\hline & CQI sub-band group size & $8 \mathrm{PRB}$ \\
\hline & PMI group size & $8 \mathrm{PRB}$ \\
\hline & HARQ & 3 re-transmissions, 8 processes \\
\hline
\end{tabular}




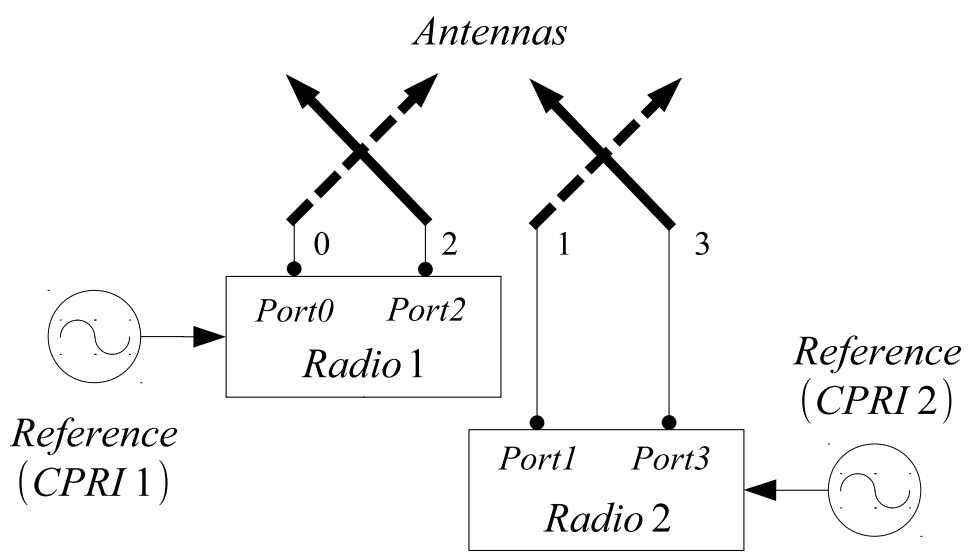

(a) Cross-Polarized Antennas (Co-Pol on Different Radios)

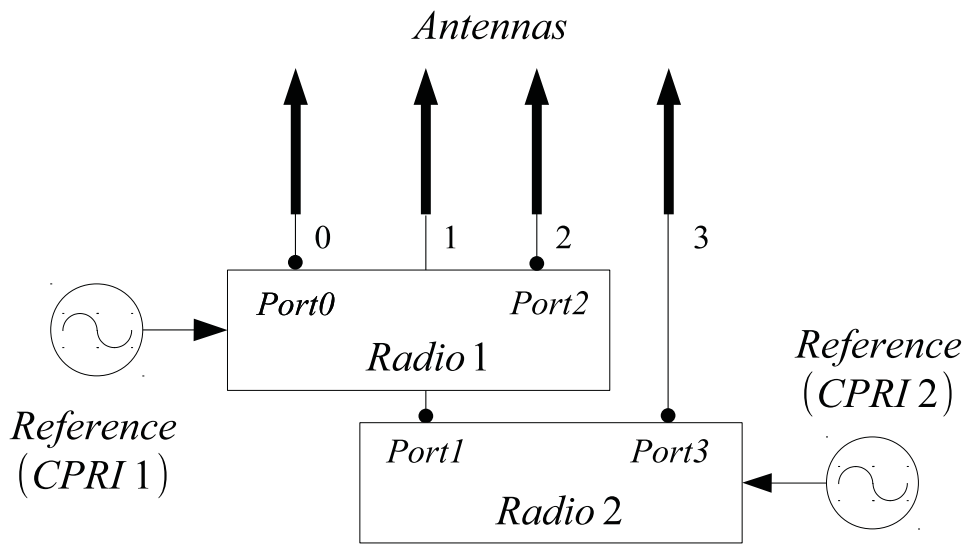

(b) Linear Array (Adjacent Elements on Different Radios)

Figure 4.22: BS Antenna Port Connections for Phase Noise Simulations 


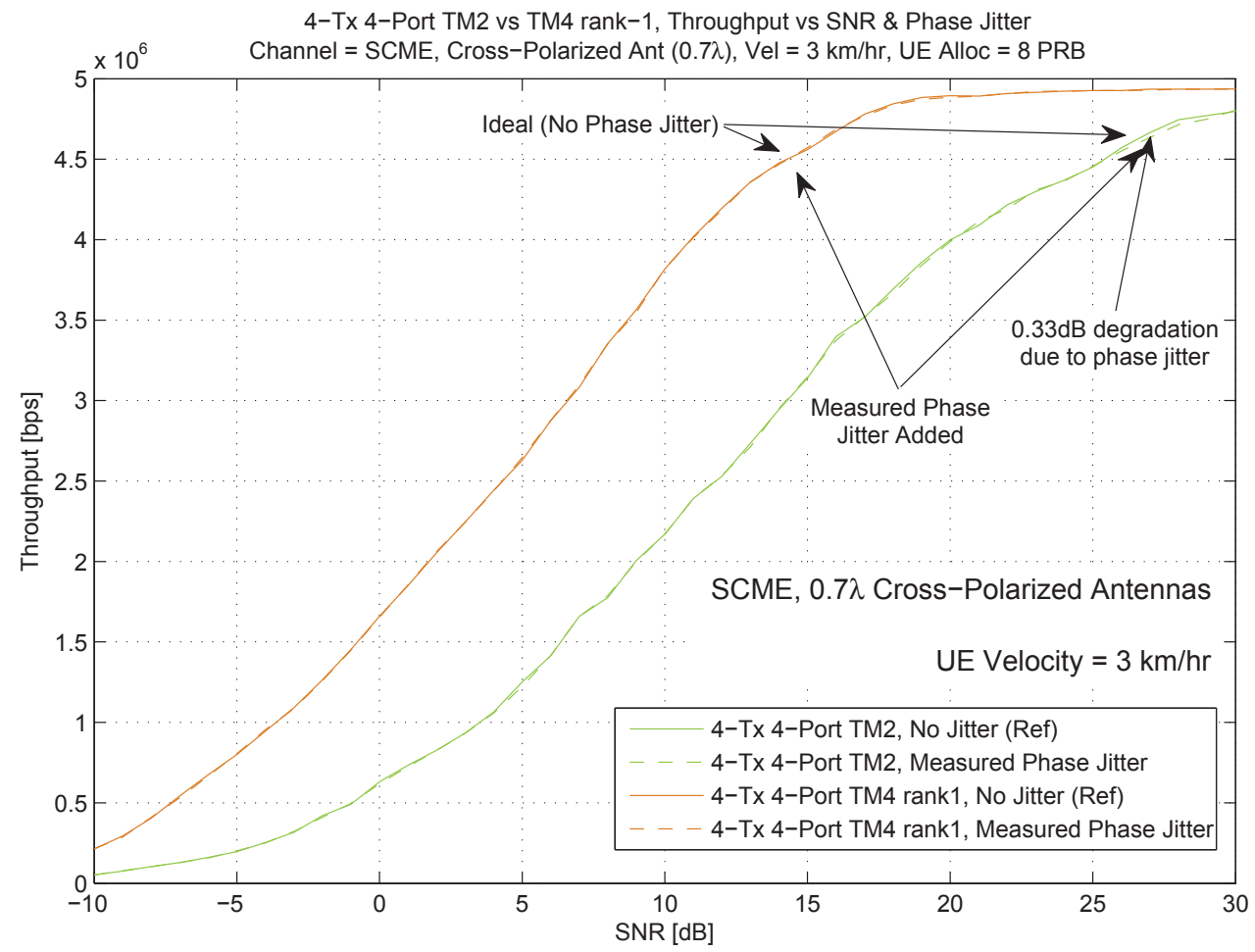

(a) Cross-Pol Antenna Spacing $0.7 \lambda$, UE Velocity $3 \mathrm{~km} / \mathrm{hr}$

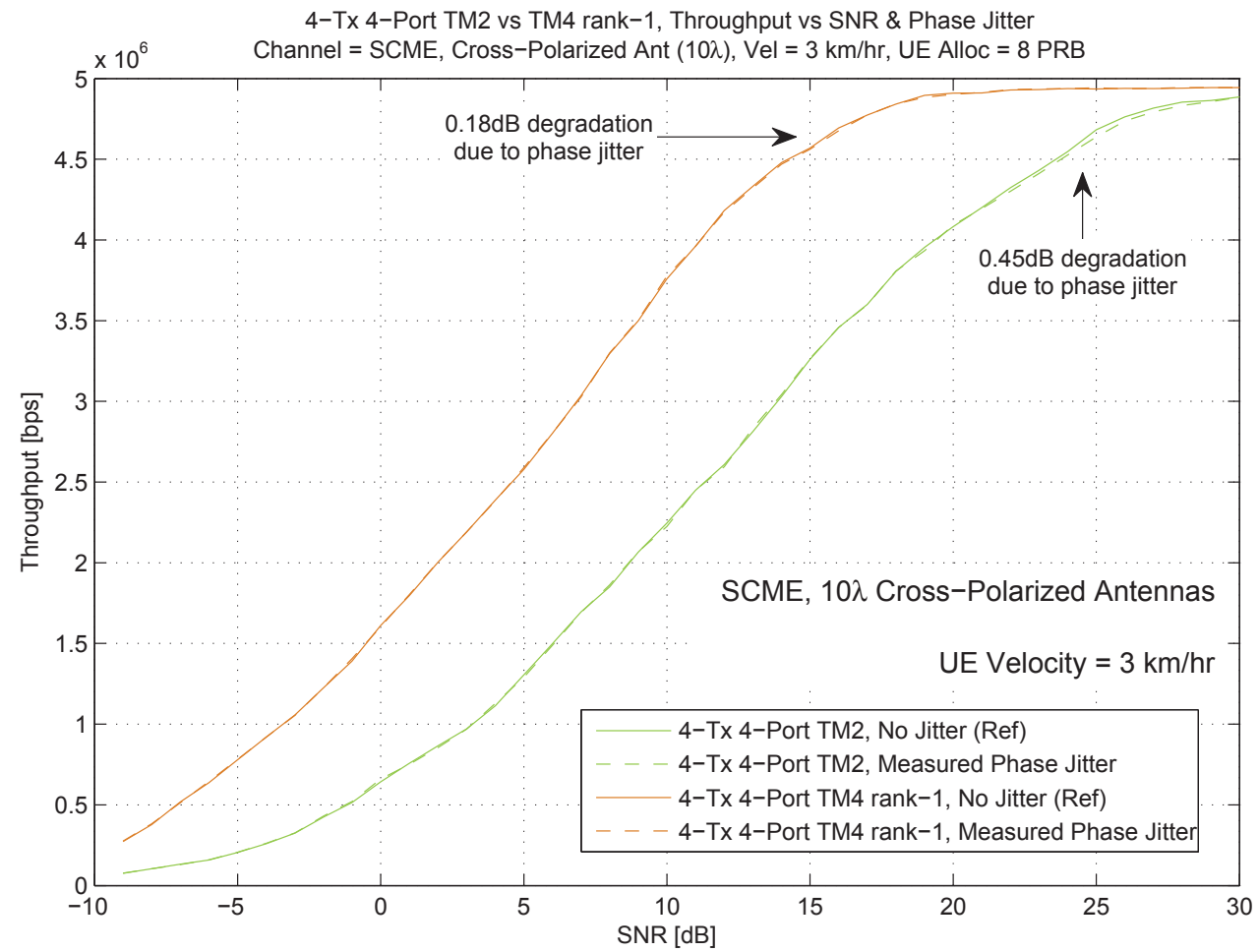

(b) Cross-Pol Antenna Spacing 10 $\lambda$, UE Velocity $3 \mathrm{~km} / \mathrm{hr}$

Figure 4.23: Phase Jitter Impairment Results on the LTE FDD Downlink in $20 \mathrm{MHz}$ of Bandwidth using Cross-polarized BS Antennas and TM2 vs TM4 rank1 


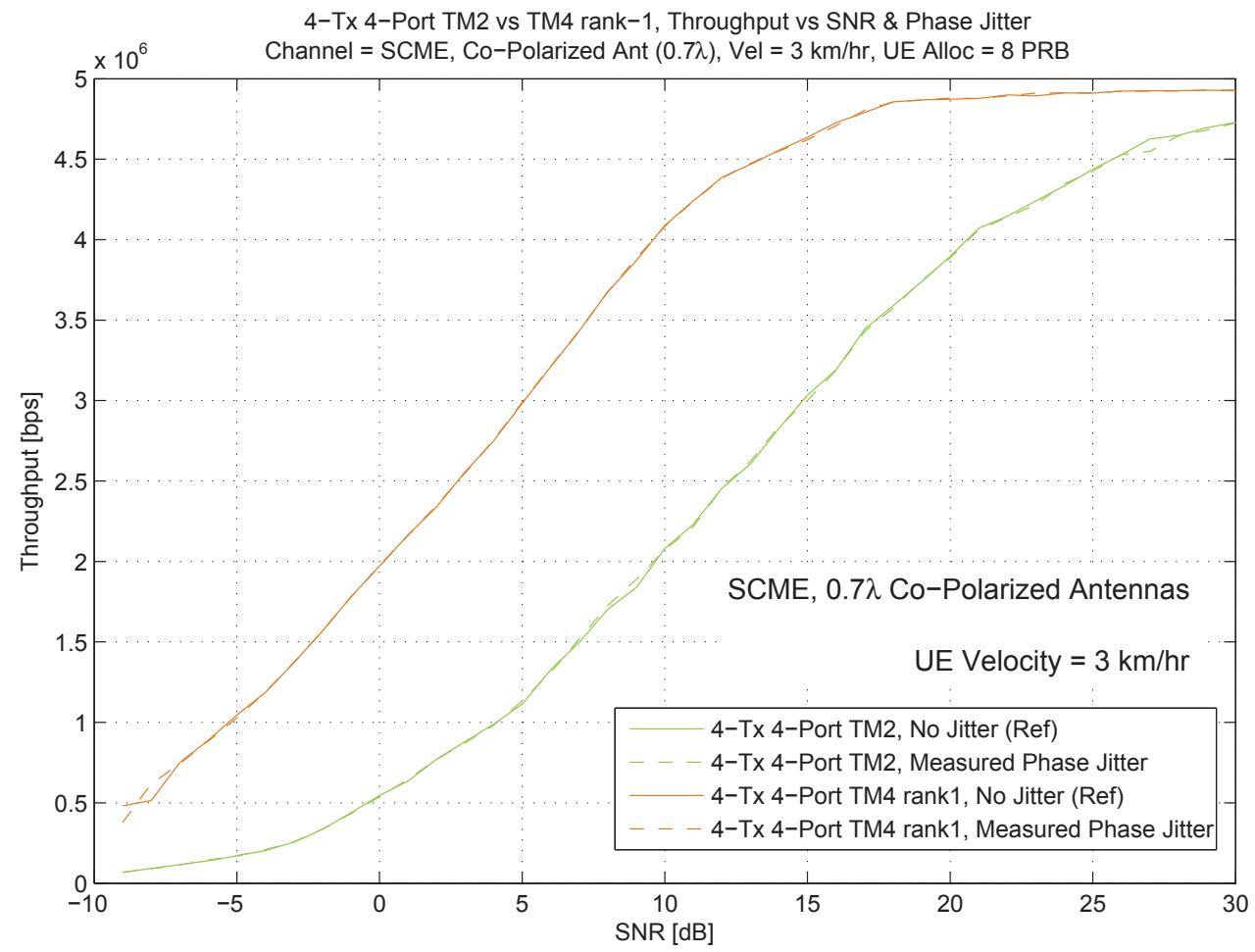

(a) Co-Pol Antenna Spacing $0.7 \lambda$, UE Velocity $3 \mathrm{~km} / \mathrm{hr}$

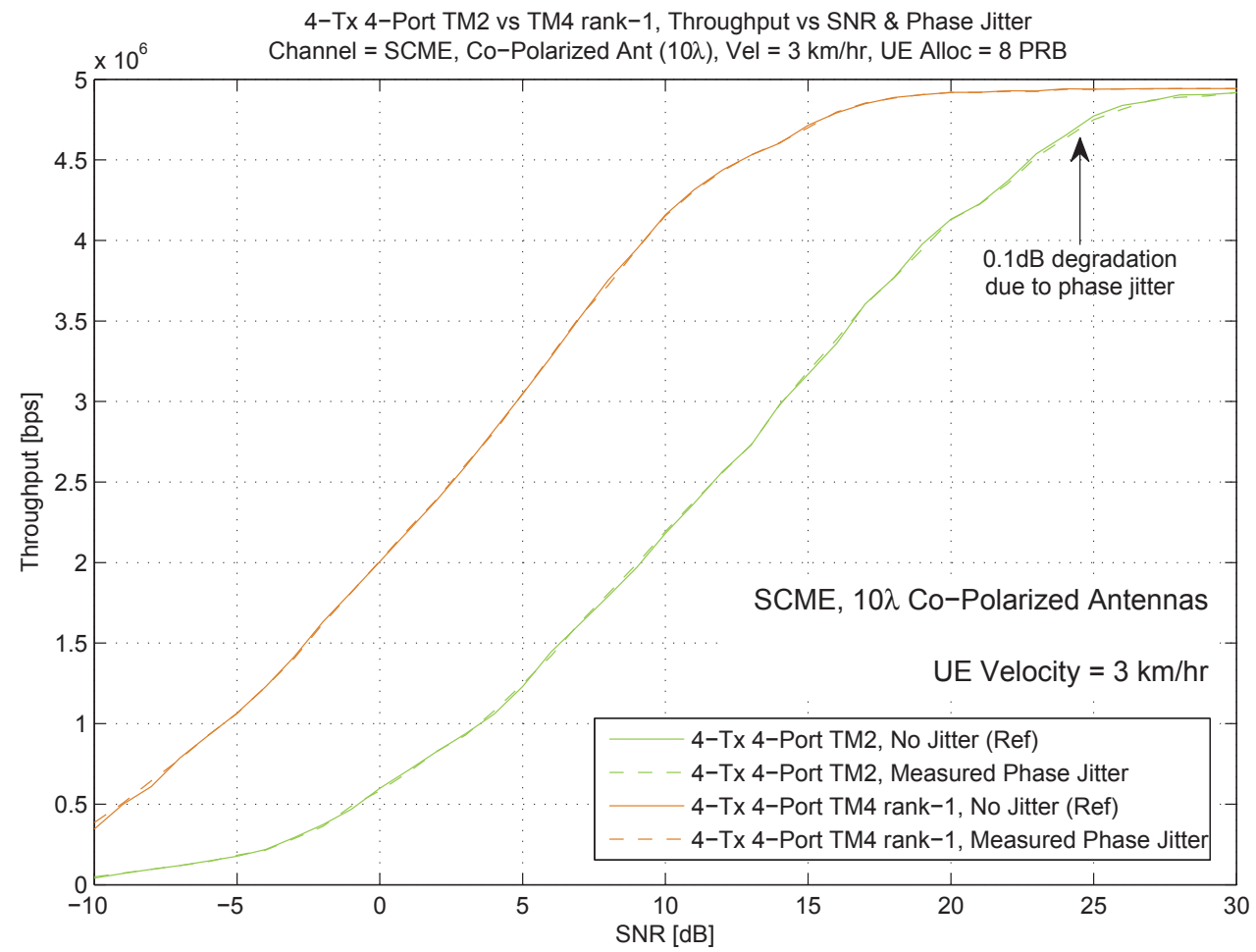

(b) Co-Pol Antenna Spacing 10 $\lambda$, UE Velocity $3 \mathrm{~km} / \mathrm{hr}$

Figure 4.24: Phase Jitter Impairment Results on the LTE FDD Downlink in $20 \mathrm{MHz}$ of Bandwidth using Co-Polarized BS Antennas and TM2 vs TM4 rank1 
The small loss of performance at high SNR is likely due to the ICI and any uncorrectable common phase error (CPE). In addition to the interference caused by the phase noise, this SNR range also typically makes use of higher modulation orders which are known to suffer due to higher sensitivity to noise. In figure 10.1 of [38], the BER curves for various MCS schemes are plotted. The modulation order used above $12 \mathrm{~dB}$ SNR is 64-ary quadrature amplitude modulation (64QAM). While the SNR at which an MCS may be used changes from design to design and even product to product due to different gains and calibration sets, the MCS curves indicates that at $20 \mathrm{~dB}$ SNR it is reasonable to expect the LTE simulator to make use of 64QAM. The use of 64QAM at and above $20 \mathrm{~dB}$ SNR is verified in simulations performed in section 4.3 .3 of this thesis where the average CQI MCS index is plotted versus SNR and the average MCS index is $10^{7}$ and up for UE velocities of 2.1 and $124.6 \mathrm{~km} / \mathrm{hr}$. The use of 64QAM by the link adaptation algorithm is justified due to the SNR of the channel however the modulation order is considerably more sensitive to noise than quadrature phase shift keying (QPSK) or 16-ary quadrature amplitude modulation (16QAM) and may contribute further to degradation.

Despite the small losses at high SNR and low velocity, the overall performance of the system when facing phase noise effects is very good and ICI does not seem to be limiting the system at high SNR, likely due to the low amplitude of the phase noise introduced by the real phase noise data sets.

The results of the ideal and phase noise impaired simulations at $120 \mathrm{~km} / \mathrm{hr}$ are given in figure $4.25 \mathrm{~b}$ and 4.26 .

Similar phase noise effects on the throughput are seen at high velocity $(120 \mathrm{~km} / \mathrm{hr})$ as in the low velocity curves. However, the degradation is very minimal across the entire SNR range for both antenna polarizations and both correlations therefore the phase noise is not considered to have a significant impact on the throughput of the system.

Finally, the phase shift occurring between the radios every $0.5 \mathrm{sec}$ does not appear to significantly impact the system as expected. LTE mobile devices perform channel estimation and analysis on each sub-frame. The receiver used to model the UE in this study is capable of synchronization every 5 sub-frames $(5 \mathrm{msec})$ where the PSS and SSS are available. In addition to synchronization, phase rotation correction is accomplished at each sub-frame based on the RS. Sample timing alignment and frequency offset correction are

${ }^{7} \mathrm{CQI}$ index 10 corresponds to 64QAM modulation as shown in the 4-bit CQI table, table A.1, in appendix A.1. 


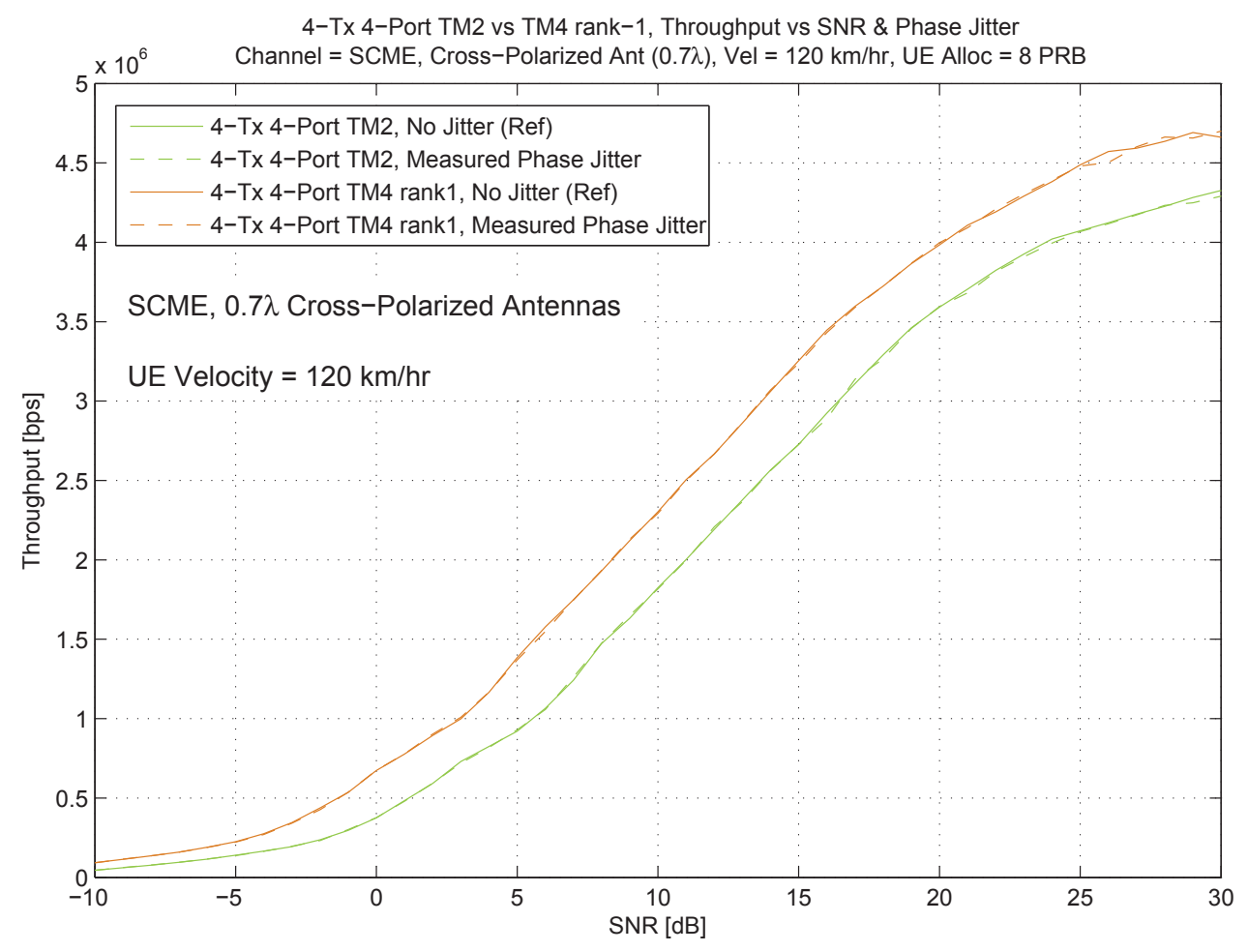

(a) Cross-Pol Antenna Spacing $0.7 \lambda$, UE Velocity $120 \mathrm{~km} / \mathrm{hr}$

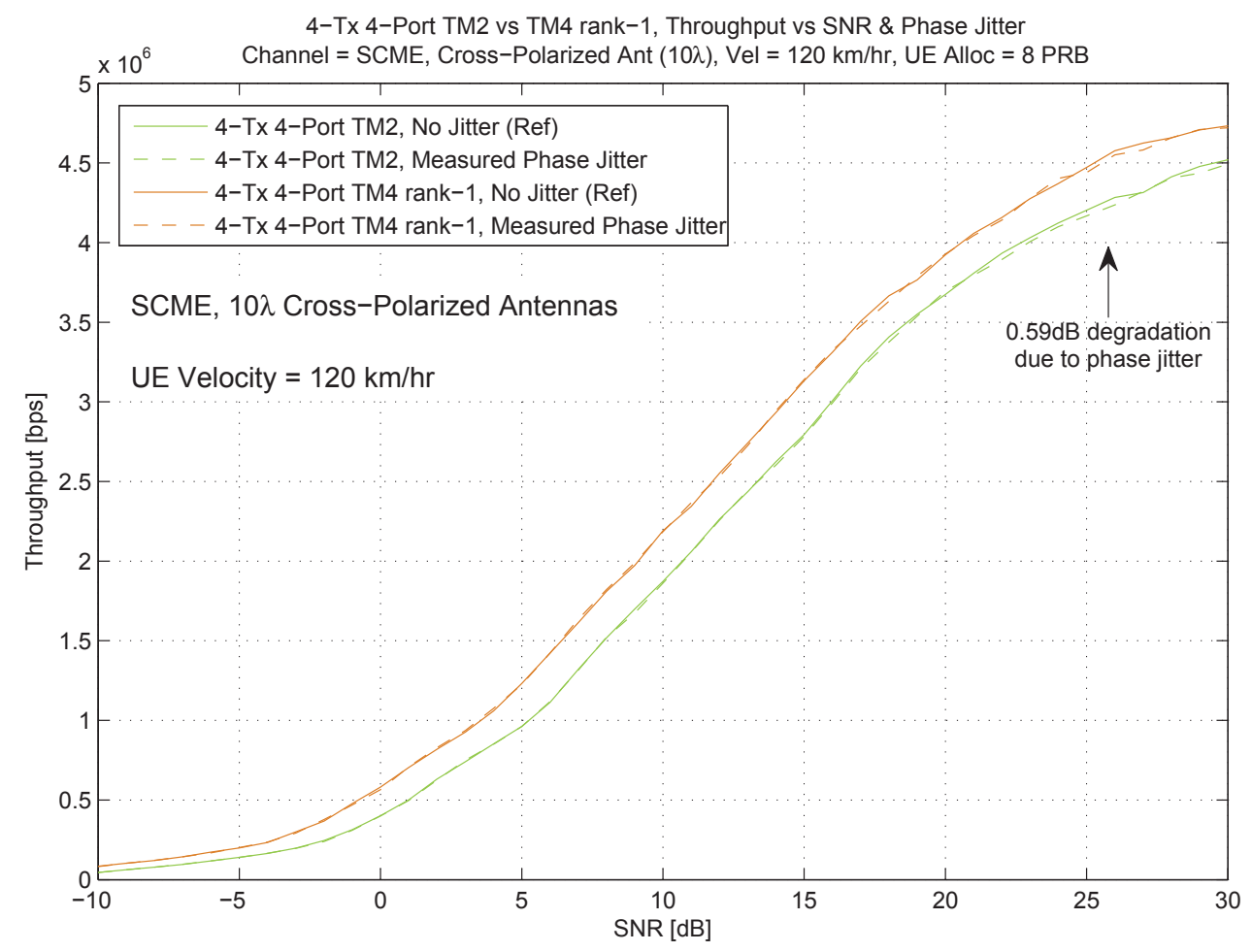

(b) Cross-Pol Antenna Spacing 10ג, UE Velocity $120 \mathrm{~km} / \mathrm{hr}$

Figure 4.25: Phase Jitter Impairment Results on the LTE FDD Downlink in $20 \mathrm{MHz}$ of Bandwidth using Cross-polarized BS Antennas and TM2 vs TM4 rank1 


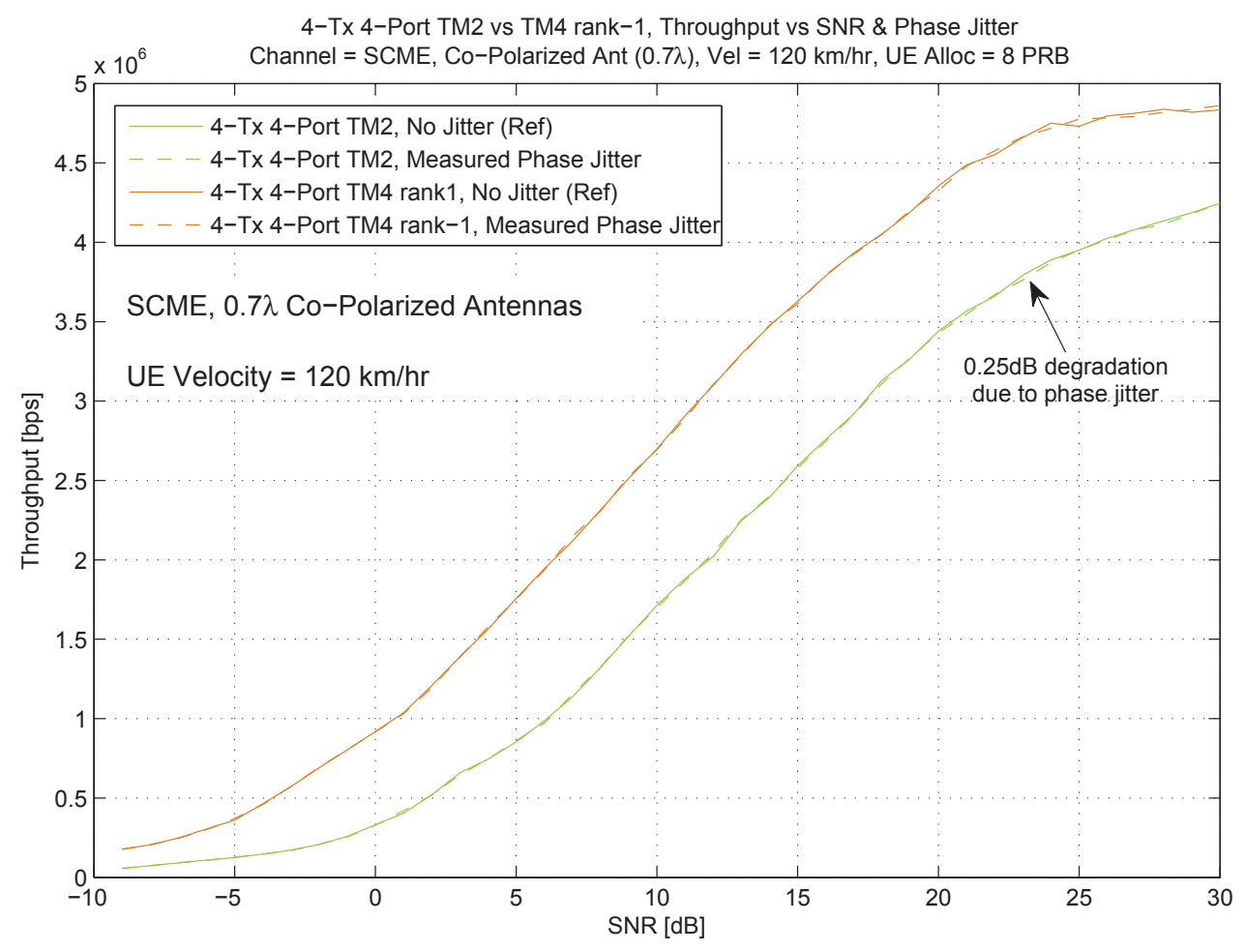

(a) Co-Pol Antenna Spacing $0.7 \lambda$, UE Velocity $120 \mathrm{~km} / \mathrm{hr}$

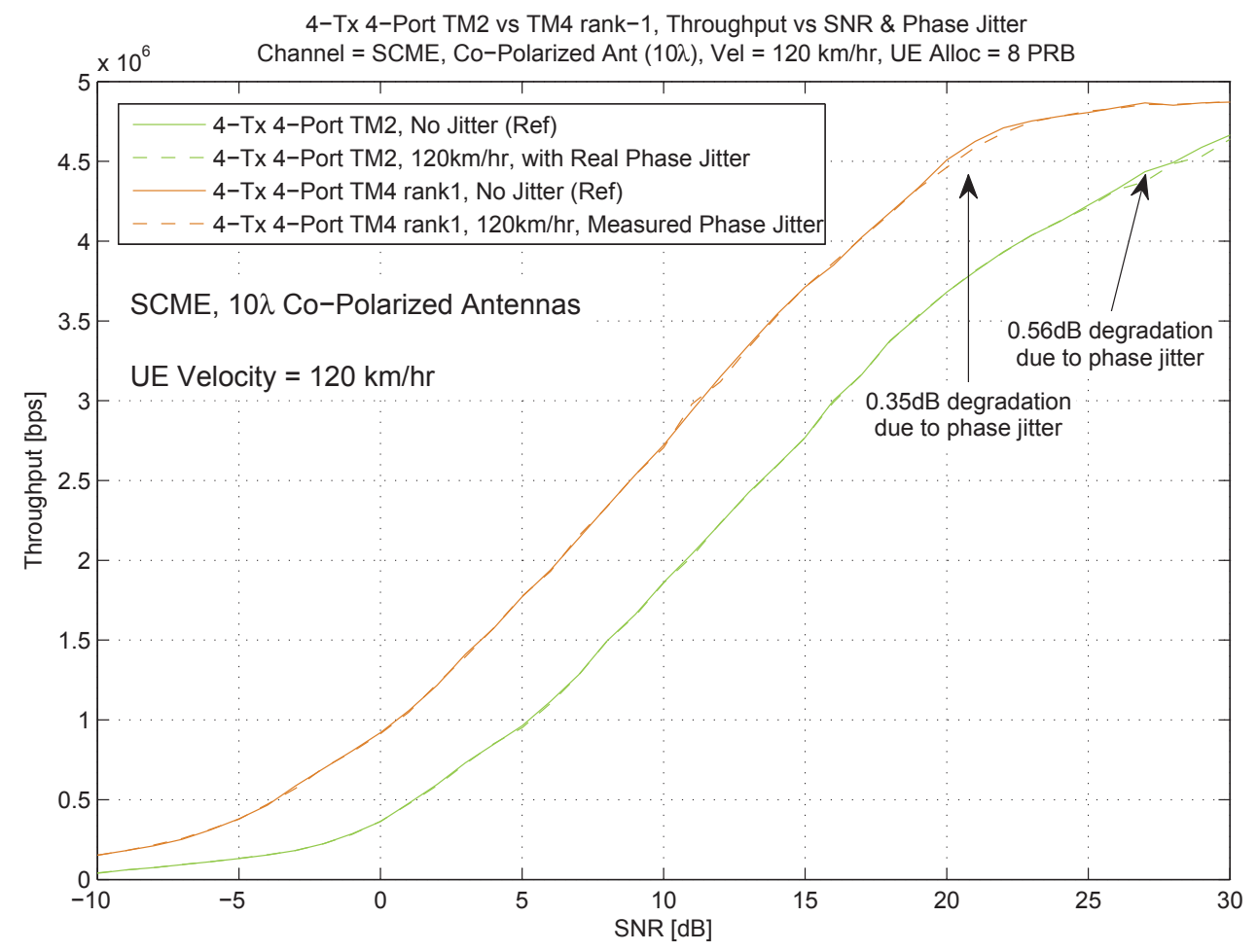

(b) Co-Pol Antenna Spacing 10 $\lambda$, UE Velocity $120 \mathrm{~km} / \mathrm{hr}$

Figure 4.26: Phase Jitter Impairment Results on the LTE FDD Downlink in $20 \mathrm{MHz}$ of Bandwidth using Cross-polarized BS Antennas and TM2 vs TM4 rank1 
frequent as well, therefore, the impact of the phase shift over the course of approximately $5.2 \mathrm{msec}$, corresponding to 6 affected sub-frames, can be progressively tracked and corrected.

\section{Phase Noise Simulation Summary}

Phase noise effects are an important topic in communication systems especially as the sampling rate and sub-carrier spacing decreases since the system quickly becomes more sensitive to the phase noise originating in the RF section or sample clock jitter before or after analog to digital or digital to analog conversion.

In this section, the simulations revealed that the level of system and LO phase noise was sufficiently low and did not affect the throughput of the LTE downlink. The $7^{\circ}$ phase shift occurring at a $2 \mathrm{~Hz}$ frequency in the real phase noise data set did not pose any problems to the communication system. The real phase noise has a standard deviation of less than half $\left(1.379^{\circ} \mathrm{RMS}\right.$ before and $1.258^{\circ} \mathrm{RMS}$ after the phase jump) of the amplitude of the generated noise on the ports representing the second radio and $0.1^{\circ} \mathrm{RMS}$ between ports on the same radio. The standard deviation of the real phase noise is considerable less than the randomly generated phase noise ( $4^{\circ}$ RMS standard deviation) applied to each port of the radios used in the reference EPA simulations. The throughput degradation observed when running the SCME simulations with real phase noise is not significant and likely the result of good system design and quiet signal sources.

These phase noise measurements and simulations show that through careful system hardware design, the phase noise encountered in practice can be kept low enough in order to avoid performance loss.

Since the cost and size of frequency references increases with the quality and purity of the device, systems and hardware engineers benefit from simulation results which help determine the impact of phase noise on the system and the required specifications of the references, PLLs, VCOs, LOs and other components making up the RF LO. 


\subsection{Effects of UE Velocity and Feedback Reporting on The LTE Downlink}

In FDD OFDM schemes, unlike CDMA, the transmit power is held constant and the system adapts to the current channel conditions by changing the modulation order, code rate, number of codewords and/or precoding to optimally match the current capacity of the channel. The CRS spread across the LTE sub-frames are used to determine the channel statistics and adapt the modulation and coding scheme to the current channel conditions. The responsibility of scheduling is placed on the eNodeB due to its central position in the LTE RAN. This allows the eNodeB to gather the channel state information from the UEs and use scheduling algorithms to try make the most efficient use of the operators spectral resources to benefit the users and the operators. The complex control loop, known as link adaptation, that calculates the MCS, precoder and rank in order to maximize the downlink transmission rate is discussed in detail in section 2.4.6.

Section 4.3.1 discusses the practical implementation issues of the LTE link adaptation control loop and its ability to track the channel as the UE velocity increases. Then, a number of simulations are presented to show the effects of UE velocity on the throughput. The first set of simulations results, shown in section 4.3.2, will show the performance degradation versus velocity using near-ideal and practical link adaptation control loop settings. Section 4.3.3 will present throughput results along with average CQI and the number of rank-2 transmissions versus SNR for a UE operating in high speed train scenario.

\subsubsection{Practical Issues of the Link Adaptation Control Loop}

As seen in the majority of the results presented in this thesis, degradation starts to occur at an earlier velocity then the standard was designed for; which is $350 \mathrm{~km} / \mathrm{hr}$ or $500 \mathrm{~km} / \mathrm{hr}$ depending on the frequency band. This is because the link adaptation control loop that is employed in an LTE network is a delicate trade-off between several aspects and as mobility increases the weaknesses of the implemented link adaptation system becomes apparent. 
Link adaptation design trade-offs:

- The number of modulation and coding schemes available to match the channel versus the CSI report overhead (number of bits).

- The CSI reporting frequency versus the amount of overhead generated by increased reporting feedback.

- The processing power, algorithm complexity at the UE and BS versus the amount of delay that will lapse before a new AMC scheme is applied.

- Measurement accuracy versus the CRS overhead in the sub-frames.

In addition to the trade-offs mentioned, the throughput is affected by the rapidly changing channel and the ability of the complex link adaptation control system to continuously match the modulation and coding scheme and precoding (if applicable) to the channel conditions in order to make the best use of the channel. Increasing UE velocity makes the job of the link adaptation control loop more difficult due to the following 4 aspects which will be discussed in more detail:

- Channel estimation accuracy

- Channel measurements errors and quantization

- Frequency of the CSI reports

- CSI report aging due to processing delays

\section{Channel Estimation Accuracy}

The first aspect is channel estimation accuracy. The sparsity of the cell-specific reference signals (CRS) affects the quality of the channel estimate in the resource blocks or subframes used to transmit the downlink data. The reference signal pattern is selected to be able to reconstruct the channel up to velocities of 350 or $500 \mathrm{~km} / \mathrm{hr}$ (based on the frequency band) but impairments, sub-optimal algorithms and a rapidly changing channel can cause the accuracy of the channel estimates between the CRS to decrease. The error between the ideal channel and the estimate causes a reduction in measured physical layer throughput.

\section{Channel Measurements Errors and Quantization}

Secondly, the accuracy of the modulation and coding scheme used for the next downlink transmission from the eNodeB is based on the imperfect channel measurements at the UE. The measurement error at the UE can propagate through the low complexity sub-optimal 
MCS and precoder selection algorithm causing less than perfect decisions. In addition, the error between the ideal transmission settings and the chosen CQI and PMI can be worsened by the CQI quantization step which reduces the modulation and coding rate to one of 14 values. Similarly, the precoder can be selected from a small set in the case of 2 antenna ports or a maximum of 16 precoders for 4 antenna ports. Finally, the errors accumulated in the channel state indicators sent to the eNodeB can cause the eNodeB scheduler to make less than optimal MCS and precoder choices since the eNodeB scheduler and the outer-loop link adaptation algorithm uses the reported information to decide the transmission settings used for future downlink transmissions.

\section{Frequency of the CSI Reports}

The third aspect of the system that causes degradation is the infrequent reporting of the channel state indicators used to report and track the channel at the eNodeB. The eNodeB can only attempt to match the downlink transmissions to the channel if it is provided the channel state indicators regularly and at a high enough frequency to track the changing channel. Unfortunately, the reporting cannot always be as high as required, especially in a busy cell, otherwise link adaptation overhead on the uplink would become overwhelming. In a deployed LTE network, the rate at which the uplink CQI and PMI reporting occurs is strictly controlled by the eNodeB and is limited to keep the uplink overhead low.

In LTE the frequency of CQI reports may be adapted by the base station based on the UE's needs but the authority remains at the eNodeB side of the radio access network. Periodic reports can be configured to be sent at intervals of \{off, 2, 5, 10, 16, 20, 32, 40, 64, $80,160\}$ msec. However, to keep CQI feedback overhead on the uplink channels (PUCCH and PUSCH) low, a practical reporting interval of 20 to $40 \mathrm{msec}$ might be used. At these reporting intervals only slowly moving UEs could be tracked accurately. The eNodeB can attempt to increase the reporting interval for faster moving UEs but the eNodeB scheduling algorithms would need to intelligently manage the control traffic on the uplink.

\section{CSI Report Aging due to Processing Delays}

Finally, practical processing delays in the LTE network cause latency in the link adaptation control loop as seen in figure 4.27. Even a small amount of delay between the channel measurement, MCS and precoder selection, the transmission of the CSI reports and the application of the MCS and precoder to the next transmission can cause the throughput to decrease if the processing and transmission delays are longer than the channel coherence 
time. If the CQI and PMI applied at the eNodeB are consistently aged and no longer representative of the channel then the downlink throughput will certainly suffer.

In this section, near-ideal and practical processing and transmission delays are used to show the effects of a reliable and aged CSI report. Processing and transmission delays of $4 \mathrm{msec}$ at the BS and $4 \mathrm{msec}$ at the UE cause the already infrequent CQI to be aged by 8 msec when it is finally applied.

\section{Unreliable CSI}

Although the reference signal patterns are chosen to be able to reproduce the channel within a sub-frame even at high UE velocity, ultimately the "bottle neck" of the system can be elsewhere. This is the case when the channel state information is aged and the reporting frequency is not sufficiently high to exactly track the channel. The results presented in this section will show that the CSI age and reporting interval can be quickly become limiting.

In the inevitable case where the CSI is aged and/or the CSI reporting frequency is insufficient to accurately track a very rapidly changing channel, then the link adaptation begins to act similar to a self-organizing network (SON). At high velocities, the link adaptation control loop is no longer tracking the rapid fluctuations of the channel but it is still capable of slowly adapting to the large-scale characteristics of the channel, such as the path loss and shadow fading, or in other words the average SNR.

\section{Maximum UE Velocity Supported by the CSI}

In the simulations in this section, a combination of two processing delays (2 and $8 \mathrm{msec}$ ) and three CSI reporting intervals $(1,10$ and $20 \mathrm{msec})$ are used to show the effects of latency and infrequent reporting of a practical link adaptation control system on the LTE downlink. An approximate maximum UE velocity can be determined using Clarkes model [5] and one of the relationships between the maximum Doppler spread $f_{m}$ and the coherence time $T_{c}$ using either 4.9 from [40], or 4.10 from [7] or the popular method which uses the geometric mean of the previous two coherence time equations, leading to 4.11 .

$$
\begin{aligned}
T_{c} & =\frac{1}{f_{m}} \\
T_{c, 50 \%} & =\frac{9}{16 \pi f_{m}}
\end{aligned}
$$



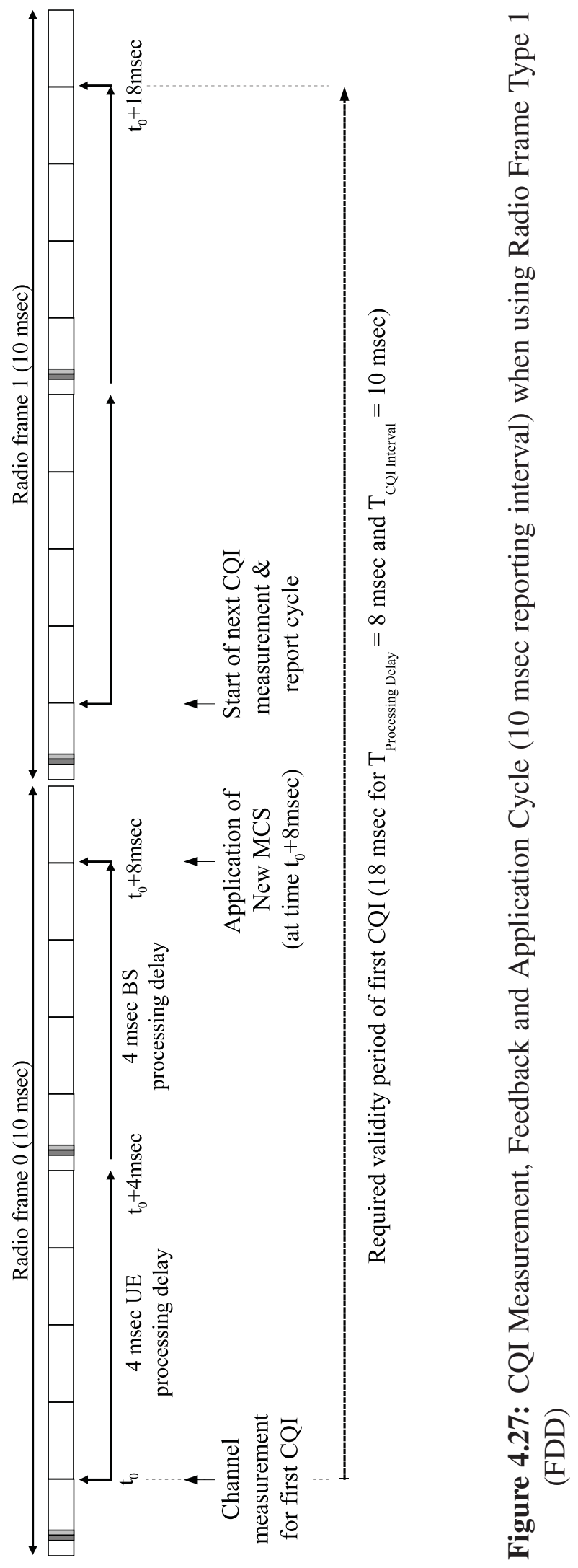


$$
T_{c, \text { geometric_mean }}=\sqrt{\frac{9}{16 \pi}} \cdot \frac{1}{f_{m}}
$$

In order for the CQI and PMI (if used) to be effective, the channel must remain relatively constant until the next channel indicators can be used by the eNodeB. Or in other words, the processing delays plus reporting interval should be shorter than the channel coherence time to ensure that the MCS and precoder are applicable when they are used at the eNodeB.

As an example, if the processing delay (UE + BS) is $8 \mathrm{msec}$ plus the time interval before the next MCS is applied is $10 \mathrm{msec}$, then the coherence time $B_{c, 50 \%}$ is required to be at least the sum of the delay plus the reporting interval. The maximum Doppler frequency $f_{m}$ can be found by rearranging the equation for the channel coherence time $T_{c, \text { geometric.mean }}$ from 4.11 as shown in equation 4.12.

$$
f_{m}=\sqrt{\frac{9}{16 \pi}} \cdot \frac{1}{T_{c, \text { geometric_mean }}}=\sqrt{\frac{9}{16 \pi}} \cdot \frac{1}{8 m s e c+10 \mathrm{msec}} \approx 23.5 \mathrm{~Hz}
$$

Using 4.13, a maximum UE velocity can be found. Based on 4.13, the UE is capable of tracking the channel variations up to approximately $10 \mathrm{~km} / \mathrm{hr}$ using the CQI for a total processing delay of $8 \mathrm{msec}$ and a reporting interval of $10 \mathrm{msec}$ at a carrier frequency of 2.6 GHz. Table 4.16 is the approximate maximum UE velocities supported by the link adaptation control loop for different link adaptation settings.

$$
v=\frac{f_{m} \cdot c}{f_{c}}=\frac{23.5 \mathrm{~Hz} \cdot 3 \cdot 10^{8} \mathrm{~m} / \mathrm{s}}{2.6 \mathrm{GHz}} \approx 2.71 \mathrm{~m} / \mathrm{s} \approx 9.8 \mathrm{~km} / \mathrm{hr}
$$

Table 4.16: Approximate Maximum UE Velocity Supported by CQI Reporting

\begin{tabular}{ccc|c}
\hline UE Processing & BS Processing & Reporting Interval & Maximum Supported \\
Delay $T_{\mathrm{d}, \text { UE }}$ & Delay $T_{\mathrm{d}, \mathrm{BS}}$ & $T_{\mathrm{CQI} \text { Interval }}$ & UE Velocity \\
\hline \hline $1 \mathrm{msec}$ & $1 \mathrm{msec}$ & $1 \mathrm{msec}$ & $58.6 \mathrm{~km} / \mathrm{hr}$ \\
$1 \mathrm{msec}$ & $1 \mathrm{msec}$ & $10 \mathrm{msec}$ & $14.6 \mathrm{~km} / \mathrm{hr}$ \\
$4 \mathrm{msec}$ & $4 \mathrm{msec}$ & $1 \mathrm{msec}$ & $19.5 \mathrm{~km} / \mathrm{hr}$ \\
$4 \mathrm{msec}$ & $4 \mathrm{msec}$ & $10 \mathrm{msec}$ & $9.8 \mathrm{~km} / \mathrm{hr}$ \\
\hline
\end{tabular}


Beyond the approximate maximum UE velocities presented in table 4.16, the UE's inability to track the rapid fluctuations of the channel will cause the system performance to degrade until the system is tracking the average SNR (assuming the average SNR is held relatively constant as the UE velocity increases). The eNodeB downlink transmissions will continue to adapt to the channel but at a relatively lower rate then the channel is changing. In some cases the CSI will be overly optimistic causing block errors and hybrid-ARQ to repeat the transmission and in other cases the CSI will be conservative and fewer bits than would be possible will be decoded. Overall, the result is a lower system performance when the CSI is unreliable. The downlink throughput is expected to plateau as the system adapts slowly to the long term trends in the channel, such as the average SNR due to path loss and shadow fading, but misses the opportunities to track the high frequency channel changes.

\subsubsection{LTE Downlink Throughput vs CSI Reporting Settings and UE Mobility Simulations}

In this section simulations results will be presented that show the effect of the UE velocity on the throughput of a UE in a single cell. The results will show how the practical implementation issues of the link adaptation system, discussed in section 2.4.6 and section 4.3.1 affects the throughput. The focus will be on the CQI and PMI reporting. The combined processing delays of the UE and $\mathrm{eNodeB}$ will be set to a near-ideal value of $2 \mathrm{msec}$ and aged value of $8 \mathrm{msec}$. The channel state indicator reporting frequency will also be set to 1 , 10 and $20 \mathrm{msec}$ to show the effect of ideal and long reporting intervals when using spatial multiplexing transmission modes.

\section{Simulation Parameters for the Throughput vs CSI Reporting Settings and UE Veloc- ity Simulations}

The channel model used for the downlink throughput simulations in this section is the 3GPP SCME with the urban macro environment and low channel angular spreading selected. The UE velocity is swept from 3 to $30 \mathrm{~km} / \mathrm{hr}$ to cover a sufficient range of velocities to see the effects of channel Doppler and link adaptation settings.

As in previous simulations, the UE is positioned at 550 meters from the eNodeB at an angle of $15^{\circ}$ from the $\mathrm{x}$-axis (the eNodeB boresight) and the UE antenna configuration is the cross-polarized antenna elements with polarizations $0^{\circ}$ and $90^{\circ}$. Although multiple drops 
within a cell or sector would better characterize the performance of the spatial multiplexing modes, the drop was considered to be reasonably representative of the UE performance based on the similarity between the 3GPP EPA curves and SCME curves from previous simulation results. Also, holding the UE position constant in the SCME simulations permits a good comparison of different transmission modes or parameter settings since the downlink simulation is subject to the same channel. The throughput degradation can be associated with a particular parameter by only changing one parameter at a time. Finally, a large number of sub-frames (15,000 sub-frames or 210,000 OFDM symbols) was simulated ensuring the results converged with good accuracy.

At the eNodeB, the antenna spacing chosen is $0.7 \lambda$ since it was shown to be the highest performing configuration in previous sections of this chapter.

The three base station antenna configurations used for simulations in this section are:

- Cross-polarized with 2 transmitters and 2 ports (fig. 4.2a).

- Cross-polarized with 4 transmitters and 2 ports (fig. $4.2 \mathrm{~b}$ ).

- Cross-polarized with 4 transmitters and 4 ports (fig. $4.2 \mathrm{~b}$ ).

The UE is allocated $8 \mathrm{PRB}$ of the $50 \mathrm{PRBs}$ available in the FDD system bandwidth to approximate a typical UE resource allocation and sub-band CQI reporting. In term of the PMI, the PMI is set to wideband reporting since it represents the majority of the PMI feedback modes for closed-loop and open-loop spatial multiplexing. Finally, the simulations are configured to focus on the degradation due to the imperfect or infrequent CQI and PMI, therefore the UE is assumed to have perfect channel knowledge (ideal channel estimation and synchronization).

The simulation parameters for the "Downlink Throughput vs CSI Reporting Settings and UE Velocity" simulations are summarized in table 4.17.

\section{Throughput vs CSI Reporting Settings and UE Velocity Simulation Results}

The downlink throughput versus UE velocity results in this section are presented as follows. The graphs in figure 4.28 represents simulations using 4 BS transmitters and 4 antenna ports, while the curves in figure 4.29 use an 4 transmitter and 2 port antenna configuration. The last set of graphs shown in figure 4.30 use 2 BS transmitters and 2 ports.

For all antenna configurations simulated (4-Tx 4-Port, 4-Tx 2-Port and 2-Tx 2-Port) 
Table 4.17: Simulation Parameters for the Downlink Throughput vs CSI Reporting Settings and UE Velocity Simulations

\begin{tabular}{|c|c|c|}
\hline Category & Parameter & Value \\
\hline \multirow{5}{*}{ System } & Bandwidth@ freq & $10 \mathrm{MHz}$ (50 PRBs)@2.6 GHz \\
\hline & Duplex mode & FDD \\
\hline & Sub-carrier spacing & $15 \mathrm{kHz}$ \\
\hline & Cyclic prefix & Normal CP $(4.7 \mu \mathrm{sec})$ \\
\hline & Duration & 15,000 sub-frames \\
\hline \multirow{2}{*}{ BS antennas } & Configurations & fig $4.1 \mathrm{c}, 4.1 \mathrm{~d}, 4.2 \mathrm{~b}, 4.2 \mathrm{c}$ \\
\hline & Spacing & $0.7 \lambda$ \\
\hline \multirow{4}{*}{ Channel } & Channel model & SCME, Urban macro \\
\hline & Angular spreading & Low \\
\hline & UE velocity & Swept from 3-30 km/hr \\
\hline & SNR & Fixed $(15 \mathrm{~dB})$ \\
\hline \multirow{3}{*}{ Resources/Scheduling } & Transmission mode(s) & TM3, TM4 (up to rank 2) \\
\hline & UE resource blocks & $8 \mathrm{PRB}$ \\
\hline & OLLA & Off \\
\hline \multirow{8}{*}{ Measurements/Reporting } & Channel estimation & Ideal \\
\hline & Synchronization & Ideal \\
\hline & RS power boosting & $3 \mathrm{~dB}$ \\
\hline & CQI reporting interval & $1,10,20 \mathrm{msec}$ \\
\hline & CQI processing delay & $(\mathrm{UE}+\mathrm{BS}) 2 \mathrm{msec}, 8 \mathrm{msec}$ \\
\hline & CQI sub-band group size & $8 \mathrm{PRB}$ \\
\hline & PMI group size & Wideband \\
\hline & HARQ & 3 re-transmissions, 8 processes \\
\hline
\end{tabular}


the highest throughput achieved for each antenna configuration is when the near-ideal CQI processing delay $(2 \mathrm{msec})$ and shortest CSI reporting interval $(1 \mathrm{msec})$ are used, as seen in figures 4.28a, 4.29a and 4.30a. The high throughput performance is expected in figures 4.28a, 4.29a and 4.30a since the processing delay is short and new precoders and MCSs are applied to the next transmission frequently and in a timely fashion. The reliable precoder and MCS applied to downlink transmissions more accurately match the channel conditions. When the CSI is timely and reported frequently the downlink transmission make the most efficient use of the channel allocated to the user while also transmitting at a rate that allows the UE to successfully decode the codewords.

When the CSI processing delays or the reporting interval is increased, the throughput performance of the system degrades as the UE velocity is increased.

When the processing delays are changed from a total delay of $2 \mathrm{msec}$ to $8 \mathrm{msec}$, the resulting curves in figures $4.28 \mathrm{~b}, 4.29 \mathrm{~b}$ and $4.30 \mathrm{~b}$ show that the aged CSI used for scheduling at the eNodeB is no longer valid and causes the throughput to degrade quickly with increasing UE velocity. The resulting degradation due to aged CQI is very clear when comparing curves reporting CSI every $1 \mathrm{msec}$ in figure 4.28a and 4.28b, figure 4.29a and 4.29b, and finally figure $4.30 \mathrm{a}$ and $4.30 \mathrm{~b}$.

On the other hand, when the CSI reporting interval increases to $10 \mathrm{msec}$ or $20 \mathrm{msec}$, simulations show that the throughput does not degrade as much when comparing the throughput curves that use a near-ideal CSI processing delay of $2 \mathrm{msec}$ to the throughput curves that use a practical processing delay of $8 \mathrm{msec}$. The throughput is degraded in both processing delay cases due to the fact that the CSI reporting frequency is already too low for UE moving at speeds above $3 \mathrm{~km} / \mathrm{hr}$.

Looking only at the practical settings in figures $4.28 \mathrm{~b}, 4.29 \mathrm{~b}$ and $4.30 \mathrm{~b}$, the difference is approximately $0.2 \mathrm{Mbps}$ between the 10 and $20 \mathrm{msec}$ curves in the linear region of the graph. This implies that there is only a small amount of gain to increasing the reporting frequency when the processing delay is practical $(8 \mathrm{msec})$.

It is possible to conclude that if effort was put into improving the throughput of the system, that simply increasing the reporting interval alone when the processing delay is aged by $8 \mathrm{msec}$ provides only a small amount of gain with a penalty of increased overhead on the uplink channels. If possible, efforts should be spent improving the total processing delay of the system since this does not result in increased CSI reporting overhead on the uplink and has the benefit of improving the throughput since the CSI used for scheduling will be more reliable. Then if the UE velocity increases and the eNodeB algorithms select 
a faster reporting interval (such as $10 \mathrm{msec}$ interval), the throughput gain will be greater since the CSI reliability will not be as limited by the long practical $8 \mathrm{msec}$ processing delay.

At very low velocity $(\leq 3 \mathrm{~km} / \mathrm{hr}$ ), all figures show that an increase in CSI reporting frequency has very little effect on the throughput. This is due to the fact that the channel changes very slowly at or below $3 \mathrm{~km} / \mathrm{hr}$. Therefore at low velocities, the system could adaptively reduce the rate of CSI reporting to reduce overhead on the uplink without a significant throughput penalty since the CSI is valid for a longer period of time.

Finally, each figure demonstrates the expected plateau which occurs as velocity increases to the point where the CSI is no longer reliably able to track the rapidly changing channel. The point where the throughput approaches the minimum in each figure represents the UE velocity at which the link adaptation control loop is only able to adjust to the long term trends.

\section{Performance of TM3 vs TM4 at Increasing Velocity}

In the 4-Tx 4-port TM4 case, the throughput is highest compared to other antenna configurations and also significantly better performing then 4-Tx 4-port TM3. The reason for this is that enough beamforming gain is achieved with the 4-Tx 4-port antenna configuration to overcome the extra reference signals transmit when using 4 antenna ports. The 4-Tx 4-port TM4 transmissions enjoy a far more flexible choice of precoders. The precoder codebook for 4-Tx 4-port TM4 contains 16 precoders generating 16 combinations of rank 1 and rank 2 beam patterns (see table 2.17) compared to 4-Tx 2-Port or 2-Tx 2-port configurations which use the smaller set (see table 2.16). Therefore, it is possible to conclude that when 4 BS transmitters are available, the optimal mode is 4-Tx 4-port TM4.

In a scenario where only 2 base station antennas are available or 2 antenna ports are used, the following observation about TM3 can be made. Both 2 and 4 transmitter TM3 used with 2 antenna ports achieves a higher throughput then TM4 when the UE is no longer stationary and the velocity increases. The reason for the better TM3 performance is that the 4 antenna elements do not provide significant beamforming gain with 2 antenna ports due to the coarse set of precoder matrices available as well as the unreliable PMI at increasing velocities. In other words, the TM3-TM4 comparison with 2-ports indicates that the fixed or cycling precoder (CDD) offered by TM3 is a better scheme then using the unreliable PMI feedback of TM4 with 2 antenna ports as the UE becomes mobile. Future work could 


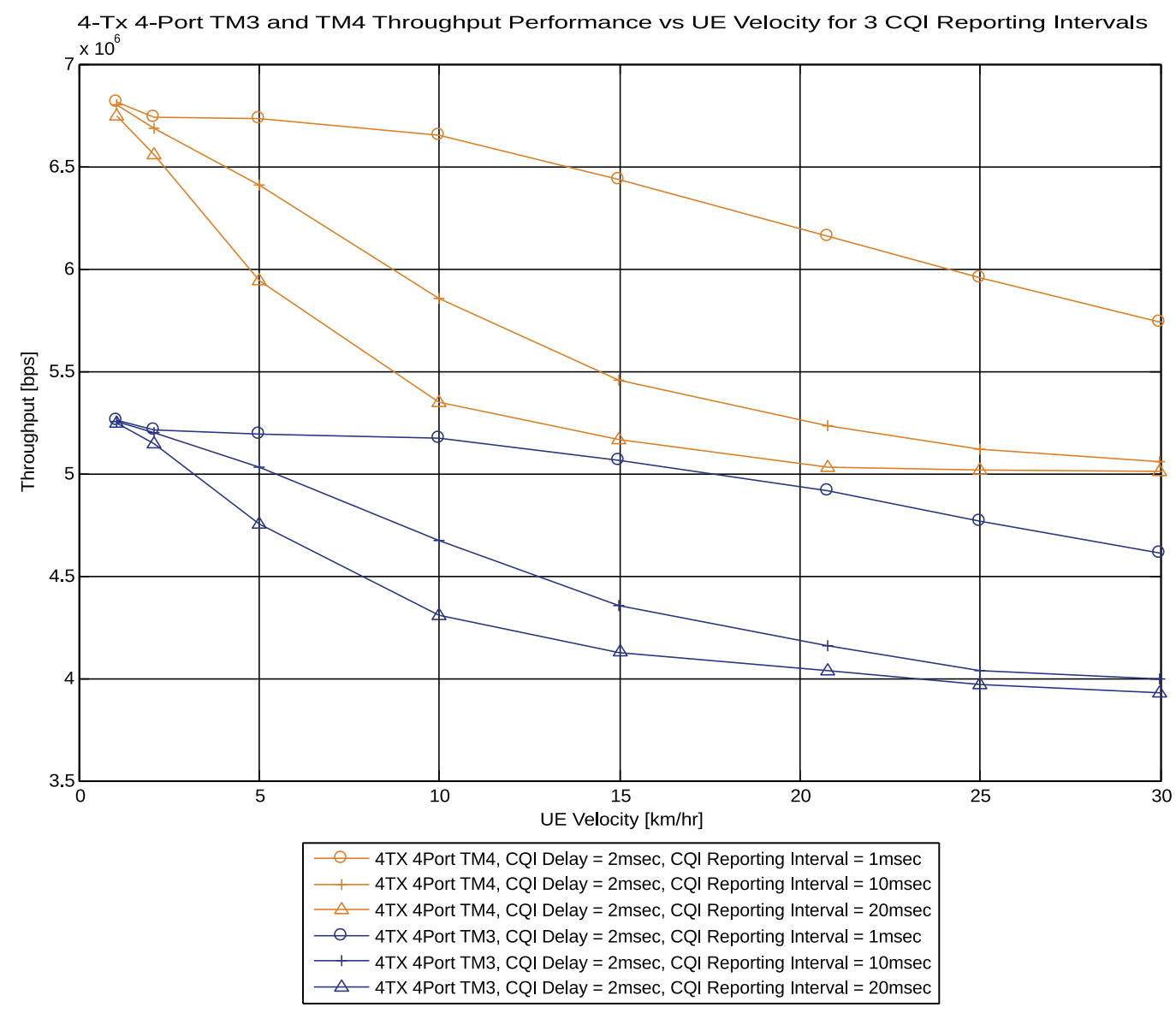

(a) Throughput vs Velocity at $15 \mathrm{~dB}$ SNR using Near Ideal CSI (CQI Delay $=2 \mathrm{msec}$ )

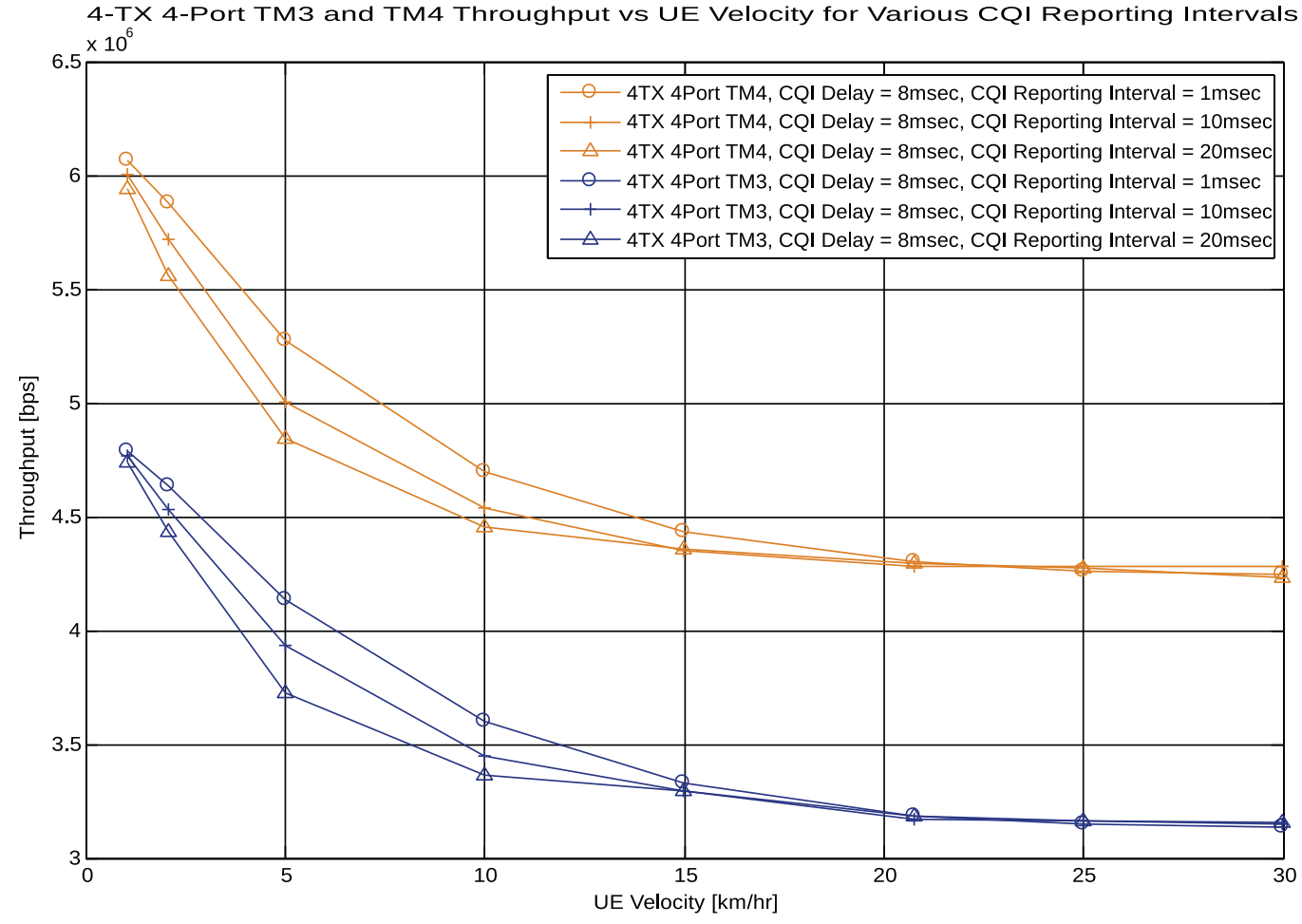

(b) Throughput vs Velocity at $15 \mathrm{~dB}$ SNR using Aged CSI (CQI Delay $=8 \mathrm{msec}$ )

Figure 4.28: 4-Tx 4-Port Throughput vs UE Velocity at $15 \mathrm{~dB}$ SNR for 3 Reporting Intervals 


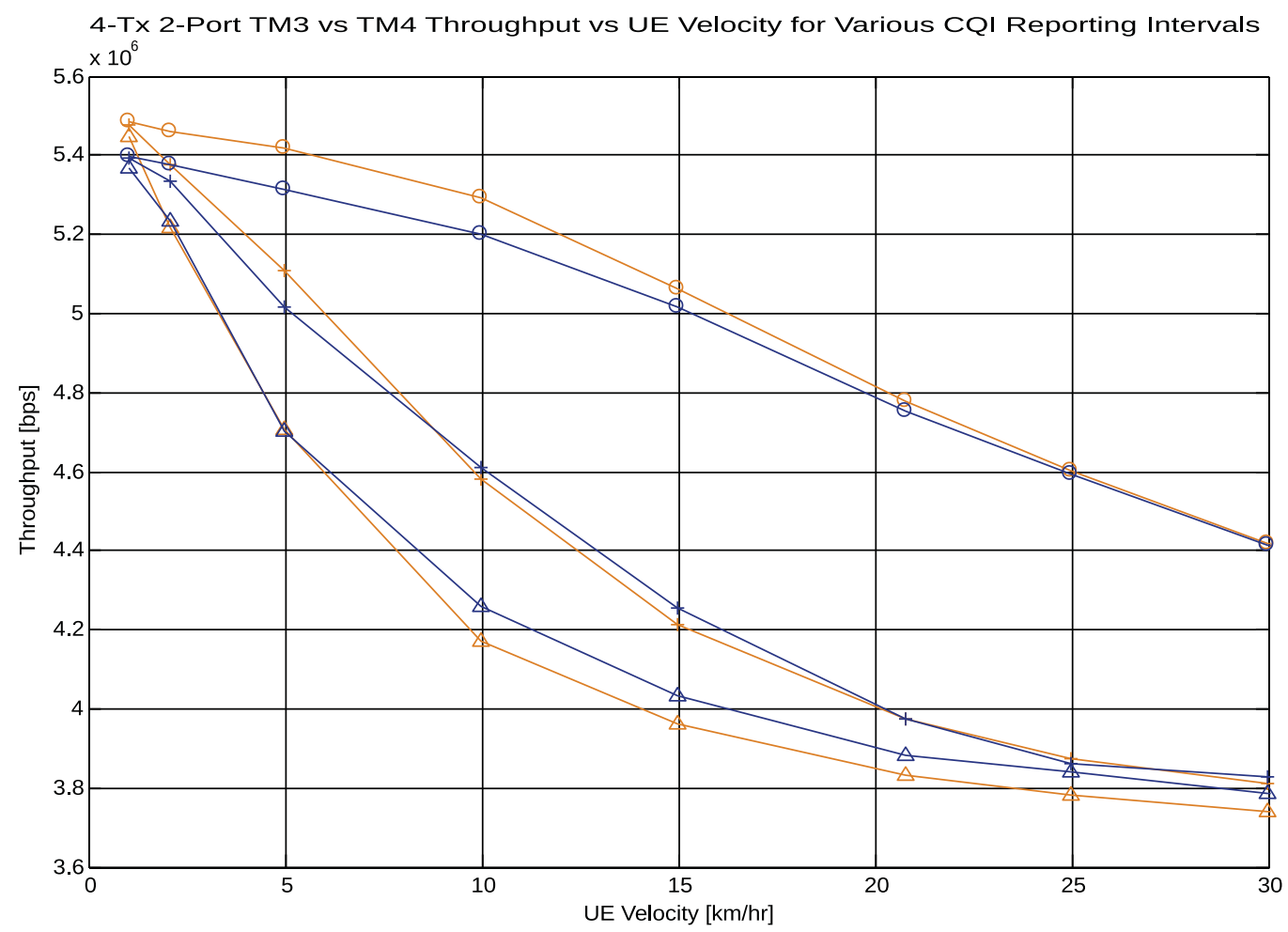

(a) Throughput vs Velocity at $15 \mathrm{~dB}$ SNR using Near Ideal CSI (CQI Delay $=2 \mathrm{msec}$ )

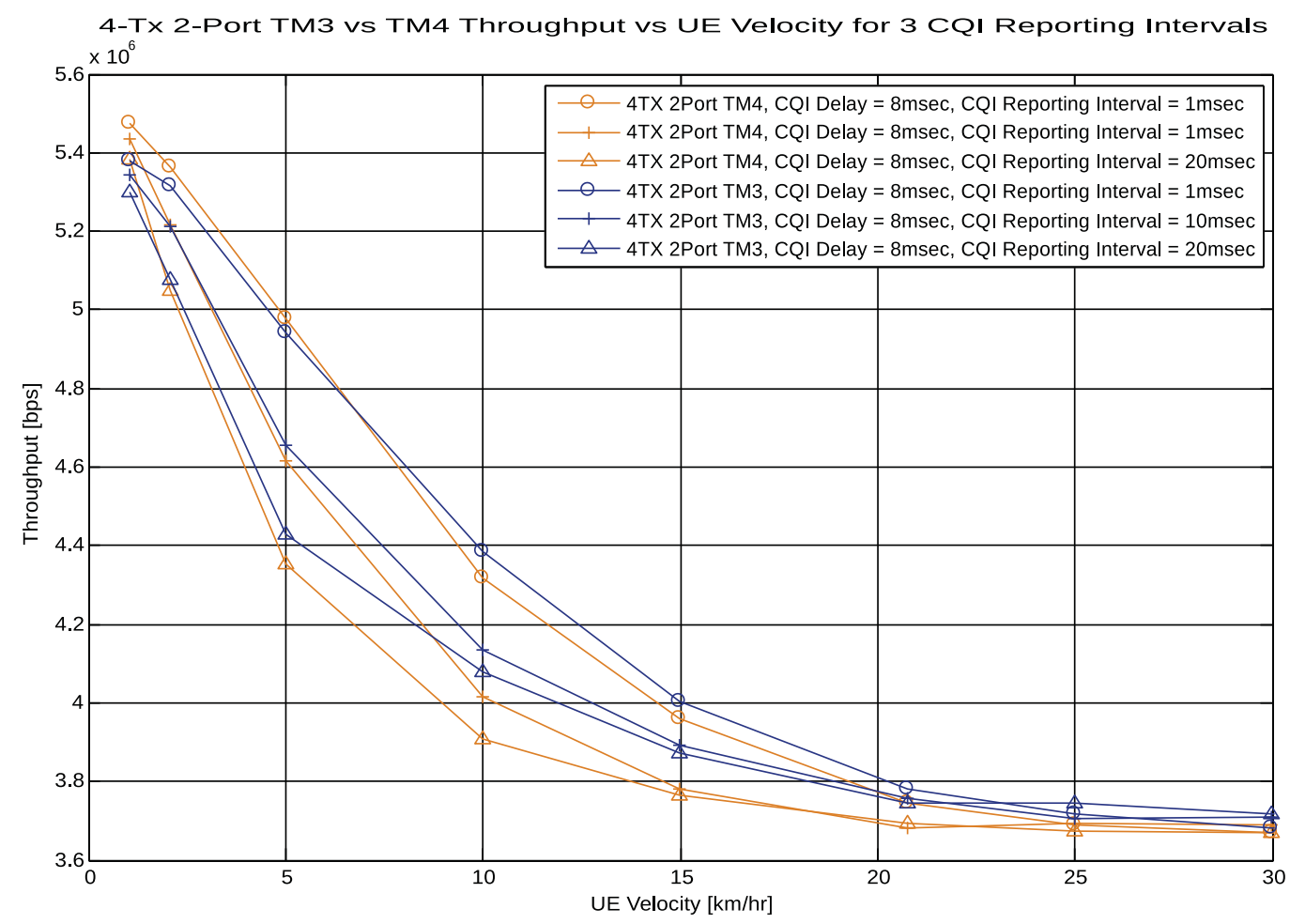

(b) Throughput vs Velocity at $15 \mathrm{~dB}$ SNR using Aged CSI (CQI Delay $=8 \mathrm{msec}$ )

Figure 4.29: 4-Tx 2-Port Throughput vs UE Velocity at $15 \mathrm{~dB}$ SNR for 3 Reporting Intervals 


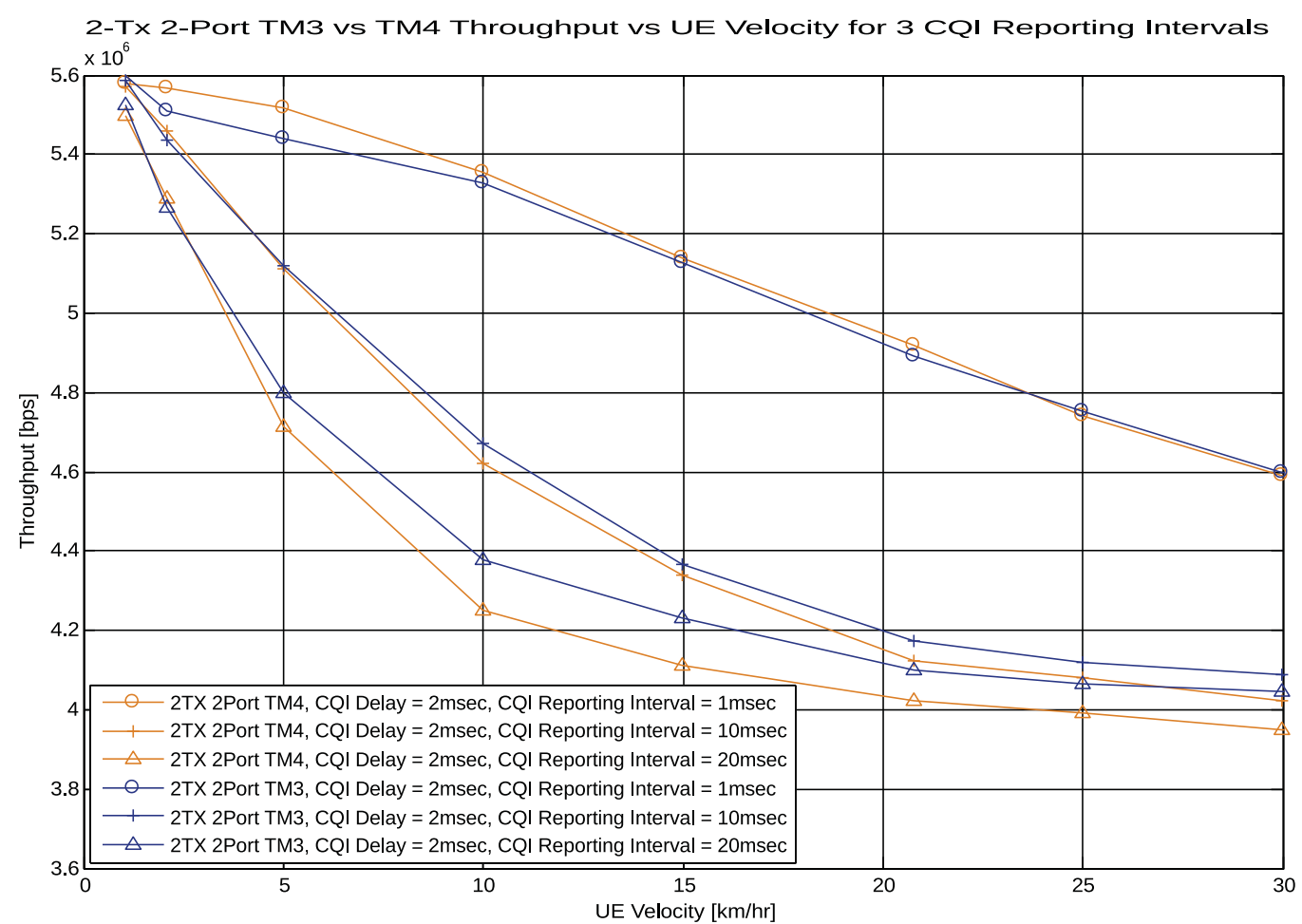

(a) Throughput vs Velocity at $15 \mathrm{~dB}$ SNR using Near Ideal CSI (CQI Delay = $2 \mathrm{msec}$ )

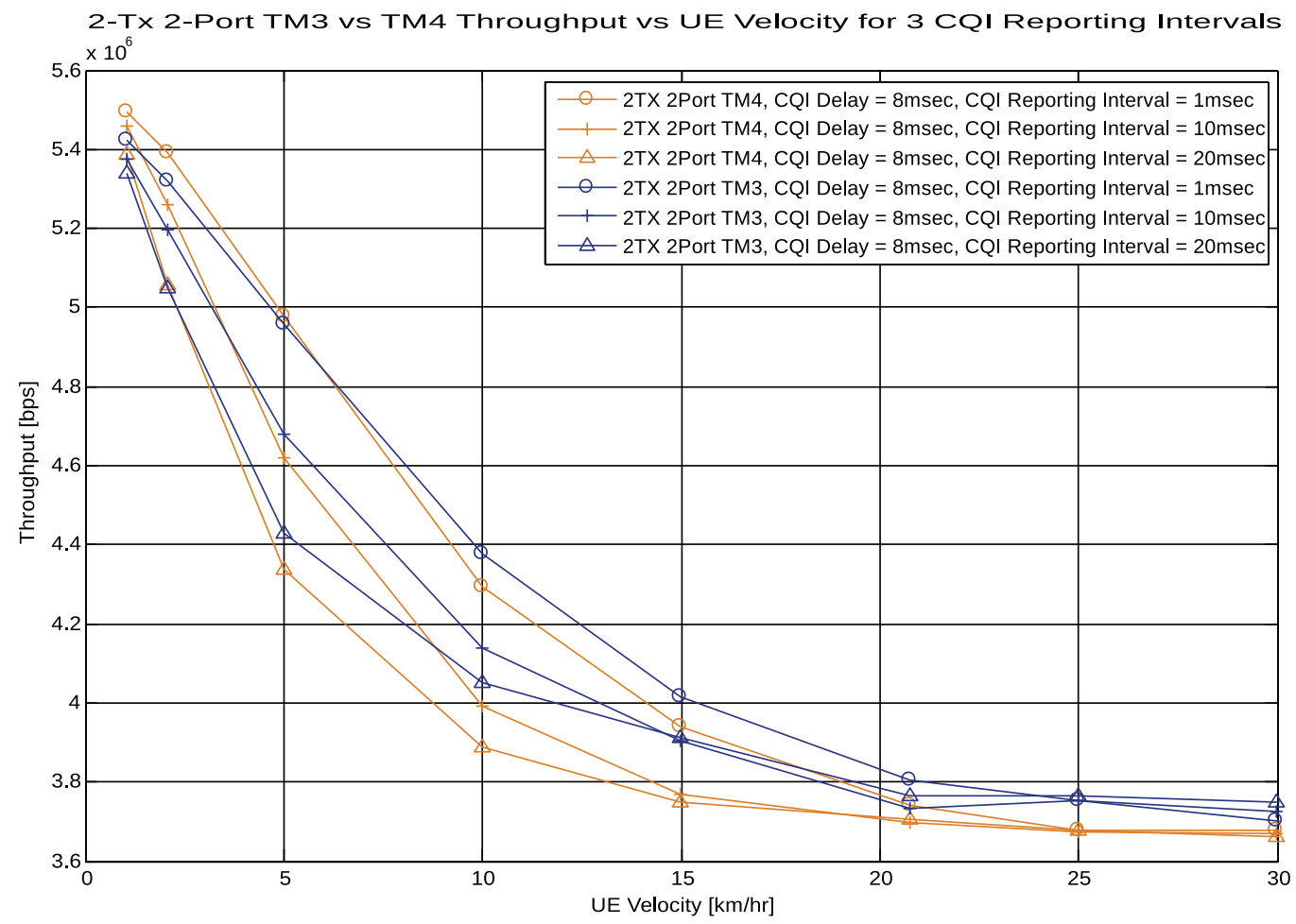

(b) Throughput vs Velocity at $15 \mathrm{~dB}$ SNR using Aged CSI (CQI Delay $=8 \mathrm{msec}$ )

Figure 4.30: 2-Tx 2-Port Throughput vs UE Velocity at $15 \mathrm{~dB}$ SNR for 3 Reporting Intervals 
include determining a method to switch between transmission modes to take advantages of TM3's higher throughput at as mobility increases when 2 transmitters and/or 2 antenna ports are used.

\subsubsection{LTE Downlink Simulations at High Speed Train Velocities}

Downlink results at very high UE velocities are of interest in order to extend LTE where people travel. Consumer demand for coverage while commuting locally or traveling vast distances is a priority for operators wanting to provide service to consumers. The high speed train on railway scenario is a particular scenario where the environment is challenging due to the extreme speeds. The resulting Doppler shift and the rate at which hand-offs occur push the capabilities of the LTE standard. However, the 3GPP members planned for LTE to be used at very high velocities by choosing an LTE sub-carrier spacing of $15 \mathrm{kHz}$. The $15 \mathrm{kHz}$ sub-carrier spacing was chosen to be large enough to handle carrier frequency offsets, caused by Doppler shifts, that approach $10 \%$ of the sub-carrier frequency spacing.

The standard also handles the issue of frequent hand-offs between base stations well. A seamless connection can be maintained at high speed train velocities due to the low latency of the control plane $(100 \mathrm{msec})$ and user plane $(5 \mathrm{msec})$ specified by the LTE standard which ensures the hand-over occurs in a shorter period of time then previous wireless technologies. If the cell size used along a high speed railway were $2 \mathrm{~km}$, for example, hand-overs would occur approximately every 14 seconds and the connection reestablishment period would be less than $1 \%$ of the possible transmission time.

\section{High Speed Train Scenario and Model}

The LTE standard provides the means to model high speed train scenarios. The reason for the specific model is due to the specific effects observed by a UE moving at a high speed train velocities along a fixed track. The environment is predominantly line of sight (LOS) and the high speeds mean the UE is rapidly passing by eNodeBs causing frequent Doppler shift reversals and hand-offs to other eNodeBs mounted along the track. The main concern with this scenario is the frequent hand-off decisions, cell acquisitions and connection reestablishments that occur and the rapid positive to negative Doppler shift that occurs as the high speed train passes by each eNodeB. 
The 3GPP standard TS 36.104 [4] presents two high speed train scenarios to model the high speed train wireless channel:

- Scenario 1: Open space

- Scenario 3: Tunnel for multi-antennas

The standard describes the two scenarios using a non-fading channel model but uses a specific Doppler shift given by equation 4.14. The Doppler shift model is found in appendix B.3 of [4] along with the specific parameters for each high speed train scenario. In equation 4.14, $f_{s}(t)$ is the Doppler shift at time $t$ affecting the signal transmit from the eNodeB and $f_{m}$ is the maximum Doppler frequency.

$$
f_{s}(t)=f_{m} \cos \theta(t)
$$

In [41], the authors used a hybrid channel model starting with scenario D2a of the WINNER work package 1 (WP1) in [42] for fading. The Doppler shift profiles from appendix B.3 of [4] are shown in figure 4.31. The Doppler shift profiles were added and the path loss models were based on measurements of a high speed passenger train from [43]. The authors refer to the resulting model as being suitable for the high speed railway.

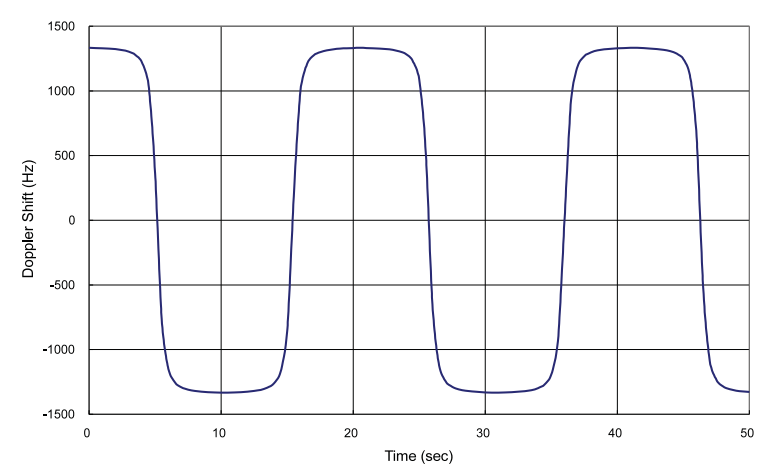

(a) Doppler Profile for Scenario 1 "Open Space"

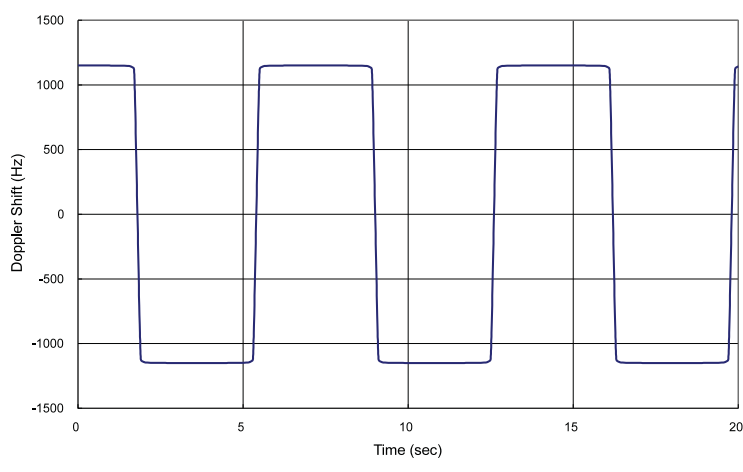

(b) Doppler Profile for Scenario 3 "Tunnel"

Figure 4.31: High Speed Train Doppler Profiles from [4]

Despite the importance of modeling the rapid change in Doppler shift from positive to negative Doppler frequencies, the following simulation do not implement the Doppler shift and the non-fading AWGN SISO model mentioned in [4]. Instead, the fading SCME urban macro model with low angular spreading was used to evaluate the high speed train results 
obtained in this study for a number of reasons. The short duration of the simulation (15 sec) would only be a light test on synchronization and carrier frequency offset algorithms based on high speed (HS) Train scenario 1 since the Doppler shift only changes 1.5 times within this period. Secondly, the Doppler shift would need to be implemented as it was not already available with the SCME urban macro model at simulation time and the need for a MIMO channel model was important in evaluating the spatial multiplexing transmission modes in question, which can achieve up to rank-2 using spatial multiplexing under proper conditions. Therefore, the SCME with UE velocities of $200 \mathrm{~km} / \mathrm{hr}$ and $350 \mathrm{~km} / \mathrm{hr}$ was assumed to be sufficient to achieve the desired results for this section.

\section{High Speed Train Simulation Parameters}

The simulations in this section were conducted to evaluate the throughput reduction experienced by a single UE traveling at high speed train velocities of 200 and $350 \mathrm{~km} / \mathrm{hr}$. Additional reference velocities of 2.1 and $124.6 \mathrm{~km} / \mathrm{hr}$ are also included to show the throughput difference between low velocity, $124.6 \mathrm{~km} / \mathrm{hr}$ and high speed train velocities. In addition to the throughput vs SNR curves, each simulation is accompanied by channel state indicator statistics. The statistics include the number of rank 2 transmissions and the average CQI index used during the simulations. Both sets of statistics are plotted against the simulated SNR to determine the effect of the rapidly changing channel on the UE's receiver performance, including channel estimation and analysis and the CSI selection algorithm.

An LTE FDD downlink simulation using $10 \mathrm{MHz}$ of bandwidth at a carrier frequency of $2.6 \mathrm{GHz}$ was performed. Spatial multiplexing transmission modes TM3 and TM4 were evaluated with 4 transmitters using 4 antenna ports and up to rank- 2 transmissions ( 2 codewords) were permitted. The UE was positioned along the track at 550 meters from the BS at an angle of $15^{\circ}$ from the antenna array bore sight direction. The BS distance from the track is 142 meters based on the previous parameter and the UE and BS heights are 1 and 30 meters respectively. The reader is referred to table 4.18 for additional details regarding the high speed train simulations.

\subsubsection{High Speed Train Results and Discussion}

The results presented in figures 4.33, 4.34, 4.35 and 4.36 are for UE velocities 2.1, 124.6, 200 and $350 \mathrm{~km} / \mathrm{hr}$ respectively. Each figure contains downlink throughput simulations 
Table 4.18: Simulation Parameters for the LTE Downlink Throughput in the High Speed Train Scenario

\begin{tabular}{|c|c|c|}
\hline Category & Parameter & Value \\
\hline \multirow{5}{*}{ System } & Bandwidth@freq & $10 \mathrm{MHz}(50 \mathrm{PRBs}) @ 2.6 \mathrm{GHz}$ \\
\hline & Duplex mode & FDD \\
\hline & Sub-carrier spacing & $15 \mathrm{kHz}$ \\
\hline & Cyclic prefix & Normal CP $(4.7 \mu s e c)$ \\
\hline & Simulation duration & 15,000 sub-frames \\
\hline \multirow{2}{*}{ BS antennas } & Configurations & fig $4.2 \mathrm{~b}$ \\
\hline & Spacing & $0.7 \lambda$ \\
\hline \multirow{4}{*}{ Channel } & Channel model & SCME, Urban macro \\
\hline & Angular spreading & Low \\
\hline & UE velocity & $2.1,124.6,200,350 \mathrm{~km} / \mathrm{hr}$ \\
\hline & SNR & Swept (-10 to $34 \mathrm{~dB})$ \\
\hline \multirow{3}{*}{ Resources/Scheduling } & Transmission mode(s) & TM3, TM4 (up to rank 2) \\
\hline & UE resource blocks & All PRBs \\
\hline & OLLA & Off \\
\hline \multirow{9}{*}{ Measurements/Reporting } & Channel estimation & MMSE \\
\hline & Synchronization & Practical $^{\mathrm{a}}$ \\
\hline & Receiver & Practical $^{\mathrm{b}}$ \\
\hline & RS power boosting & $3 \mathrm{~dB}$ \\
\hline & CQI reporting interval & $1 \mathrm{msec}$ \\
\hline & CQI processing delay & $(\mathrm{UE}+\mathrm{BS}) 8 \mathrm{msec}$ \\
\hline & CQI sub-band group size & Wideband \\
\hline & PMI group size & Wideband \\
\hline & HARQ & 3 re-transmissions, 8 processes \\
\hline
\end{tabular}

${ }^{a}$ Practical synchronization uses known reference signals in the received sub-frames to estimate the offsets.

b Practical receiver front end impairments include phase noise (1.5 ${ }^{\circ} \mathrm{RMS}$ and bandwidth of $\left.10 \mathrm{kHz}\right), \mathrm{I}-\mathrm{Q}$ imbalance (magnitude of $0.5 \mathrm{~dB}$ and phase $1.5^{\circ}$ ). 


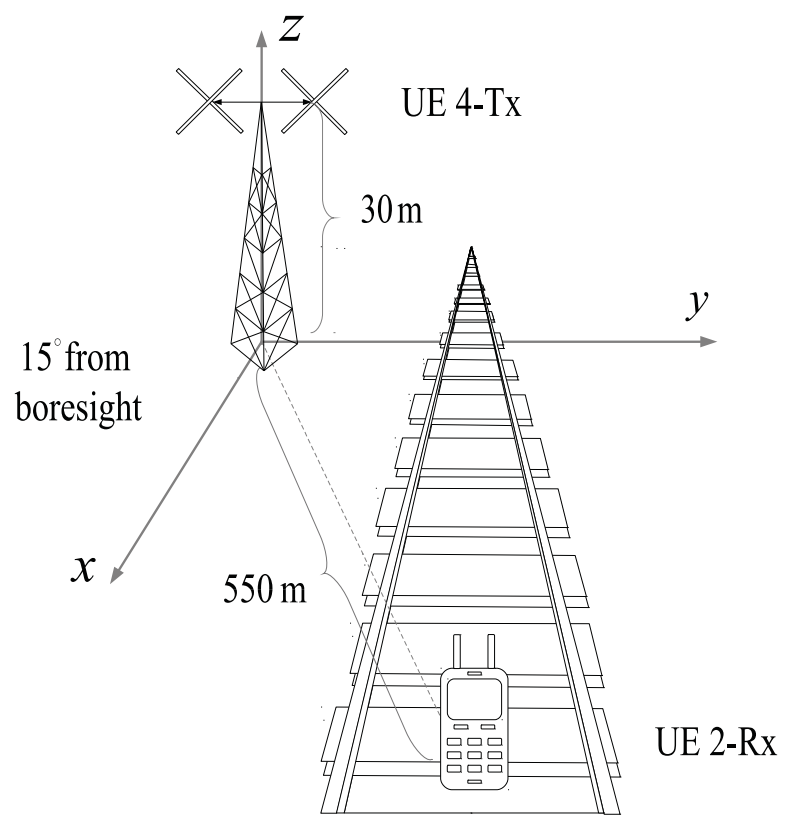

Figure 4.32: High Speed Train Physical Scenario for Simulation

along with the corresponding average CQI index and distribution of rank-2 transmissions versus SNR.

The high speed train simulations clearly show throughput decreases as the velocity of the train increases. Table 4.19 is a summary of the throughput at the four simulated velocities.

Table 4.19: Downlink Throughput at Increasing UE Velocity

\begin{tabular}{c|cc|cc}
\hline UE & \multicolumn{2}{|c}{ Throughput at $10 \mathrm{~dB}$ SNR } & \multicolumn{2}{c}{ Throughput at 34 dB SNR } \\
Velocity & TM3 & TM4 & TM3 & TM4 \\
\hline \hline $2.1 \mathrm{~km} / \mathrm{hr}$ & $18.4 \mathrm{Mbps}$ & $24.4 \mathrm{Mbps}$ & $52.4 \mathrm{Mbps}$ & $58.4 \mathrm{Mbps}$ \\
$124.6 \mathrm{~km} / \mathrm{hr}$ & $11.8 \mathrm{Mbps}$ & $16.2 \mathrm{Mbps}$ & 43.9 Mbps & 44.9 Mbps \\
$200 \mathrm{~km} / \mathrm{hr}$ & $11.1 \mathrm{Mbps}$ & $15.5 \mathrm{Mbps}$ & 33.4 Mbps & $33.4 \mathrm{Mbps}$ \\
$350 \mathrm{~km} / \mathrm{hr}$ & $9.3 \mathrm{Mbps}$ & $13.0 \mathrm{Mbps}$ & $18.0 \mathrm{Mbps}$ & $20.8 \mathrm{Mbps}$ \\
\hline
\end{tabular}

The peak throughput measured at $34 \mathrm{~dB}$ SNR drops by approximately $10 \mathrm{Mbps}$ at each 


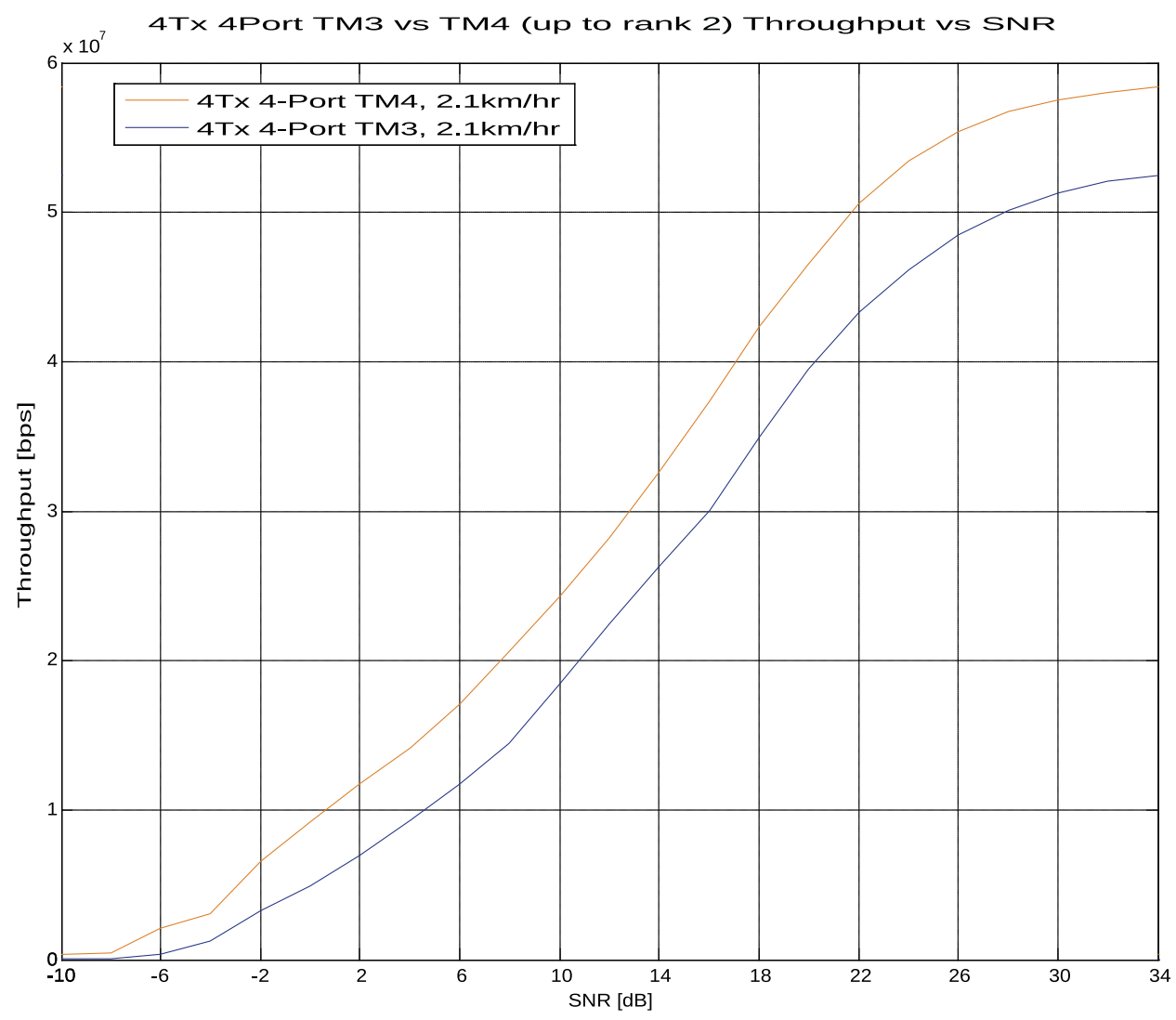

(a) Throughput vs SNR at $2.1 \mathrm{~km} / \mathrm{hr}$
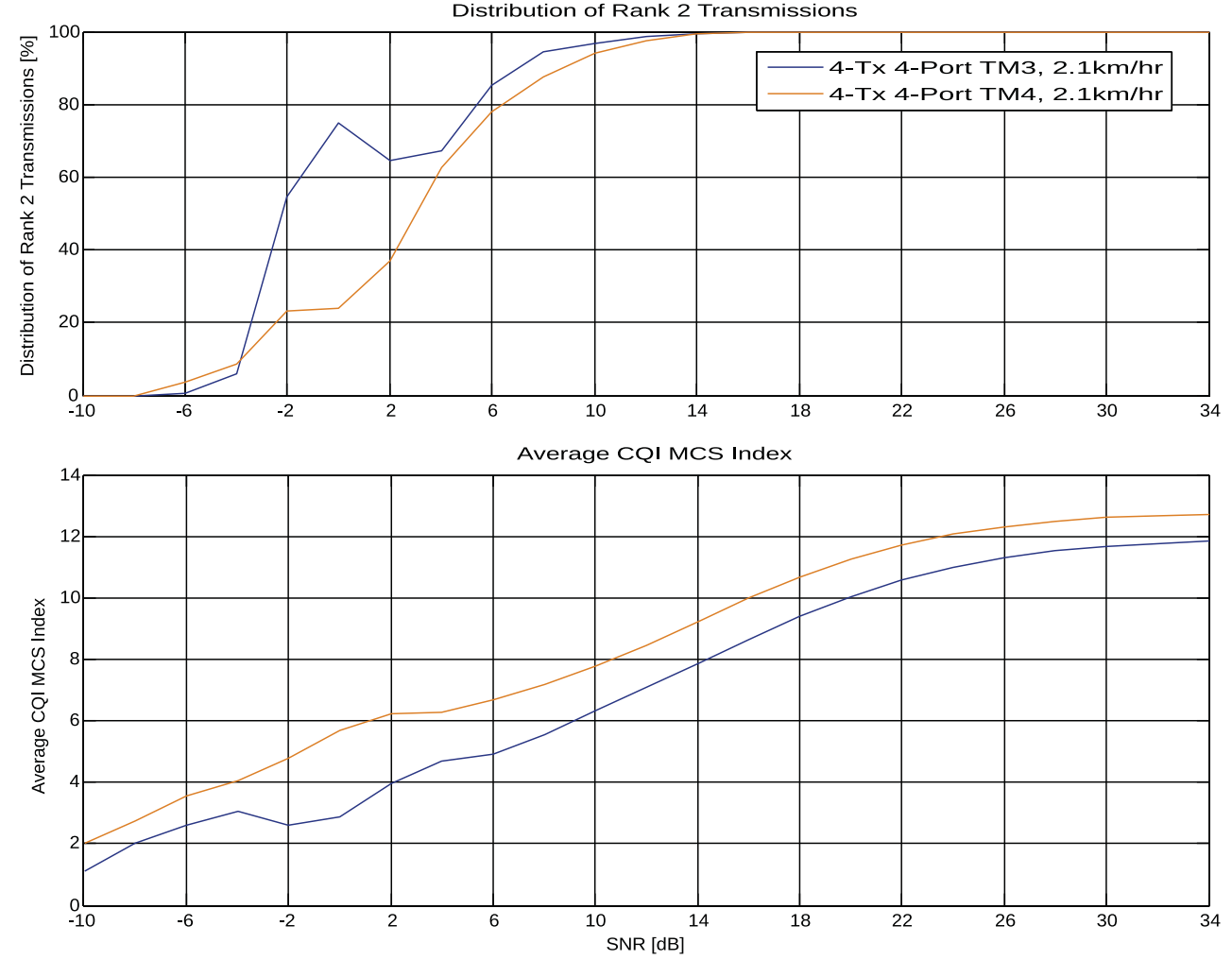

(b) Rank Distribution and Average CQI MCS Index at $2.1 \mathrm{~km} / \mathrm{hr}$ 


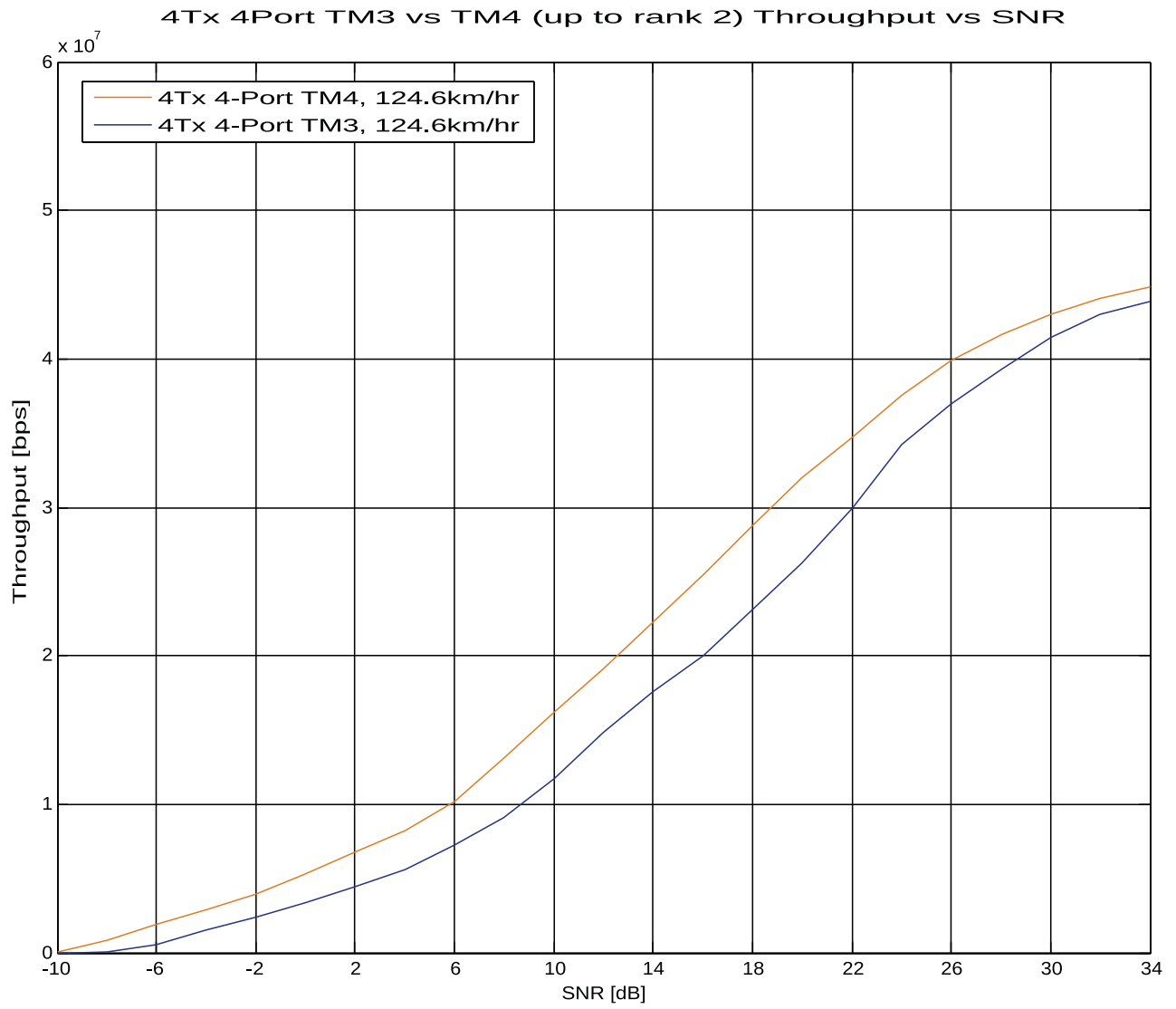

(a) Throughput vs SNR at $124.6 \mathrm{~km} / \mathrm{hr}$

Distribution of Rank 2 Transmissions
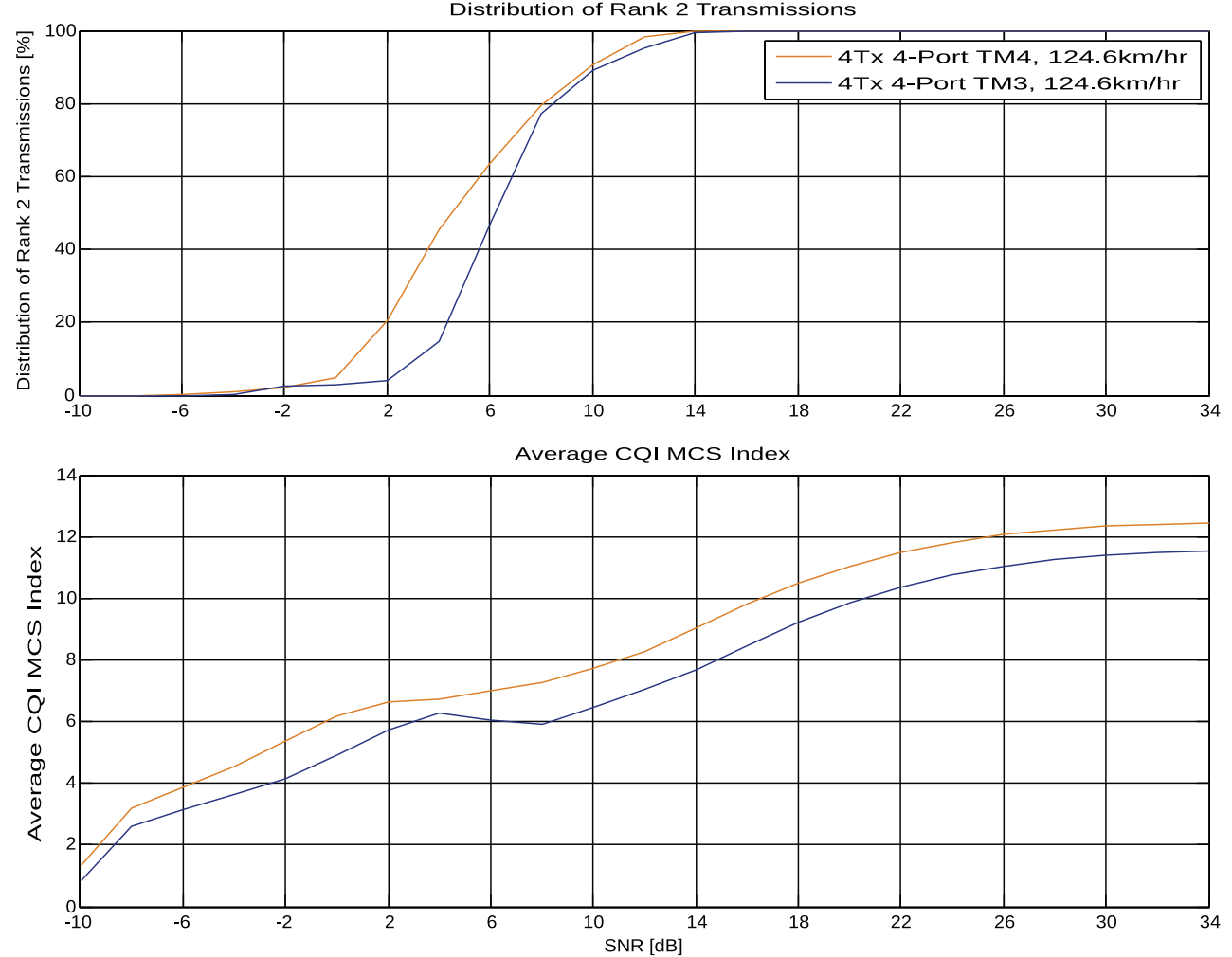

(b) Rank Distribution and Average CQI MCS Index at $124.6 \mathrm{~km} / \mathrm{hr}$

Figure 4.34: 4-Tx 4-Port High Speed Train Simulation Results at $124.6 \mathrm{~km} / \mathrm{hr}$ 


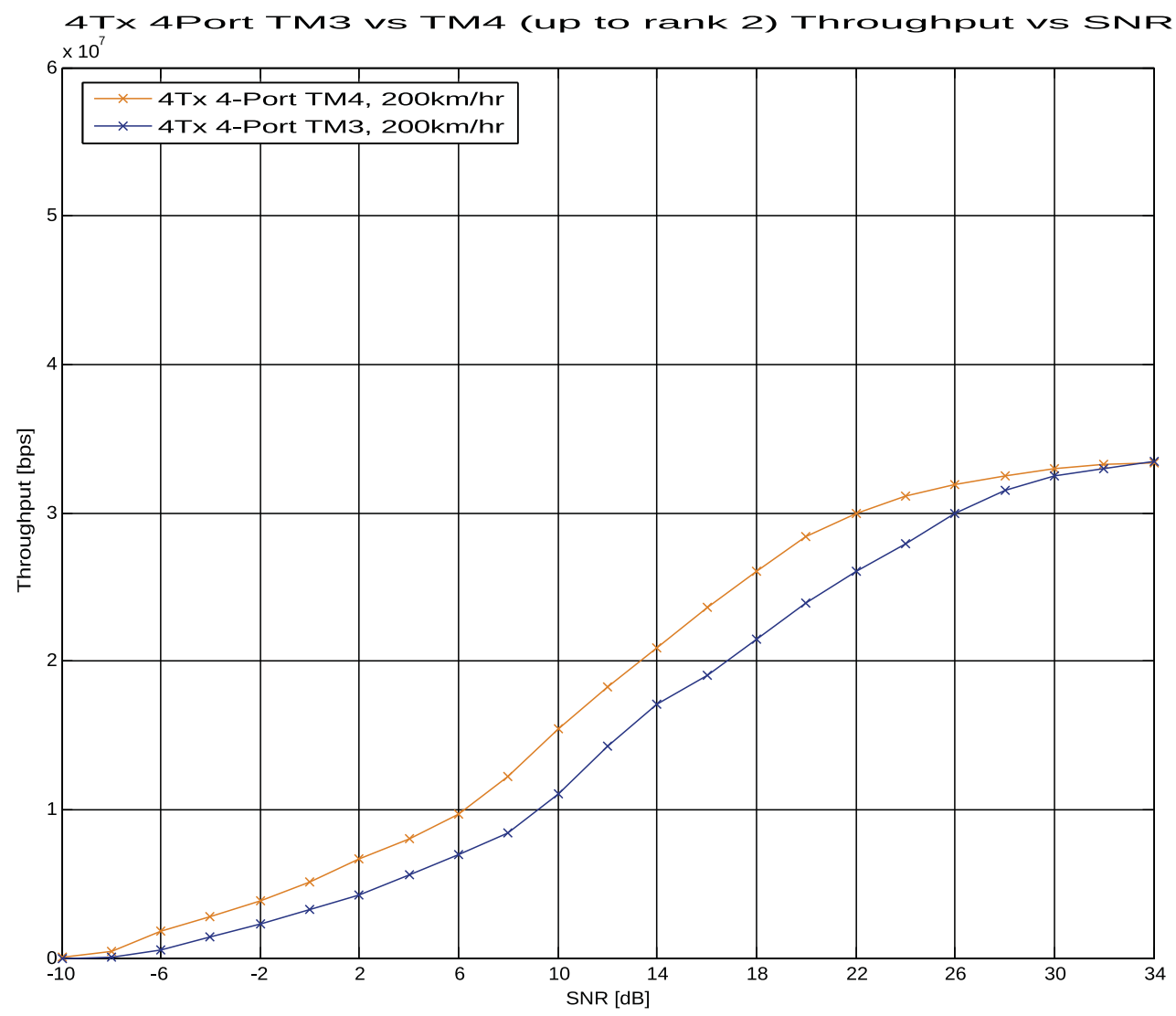

(a) Throughput vs SNR at $200 \mathrm{~km} / \mathrm{hr}$
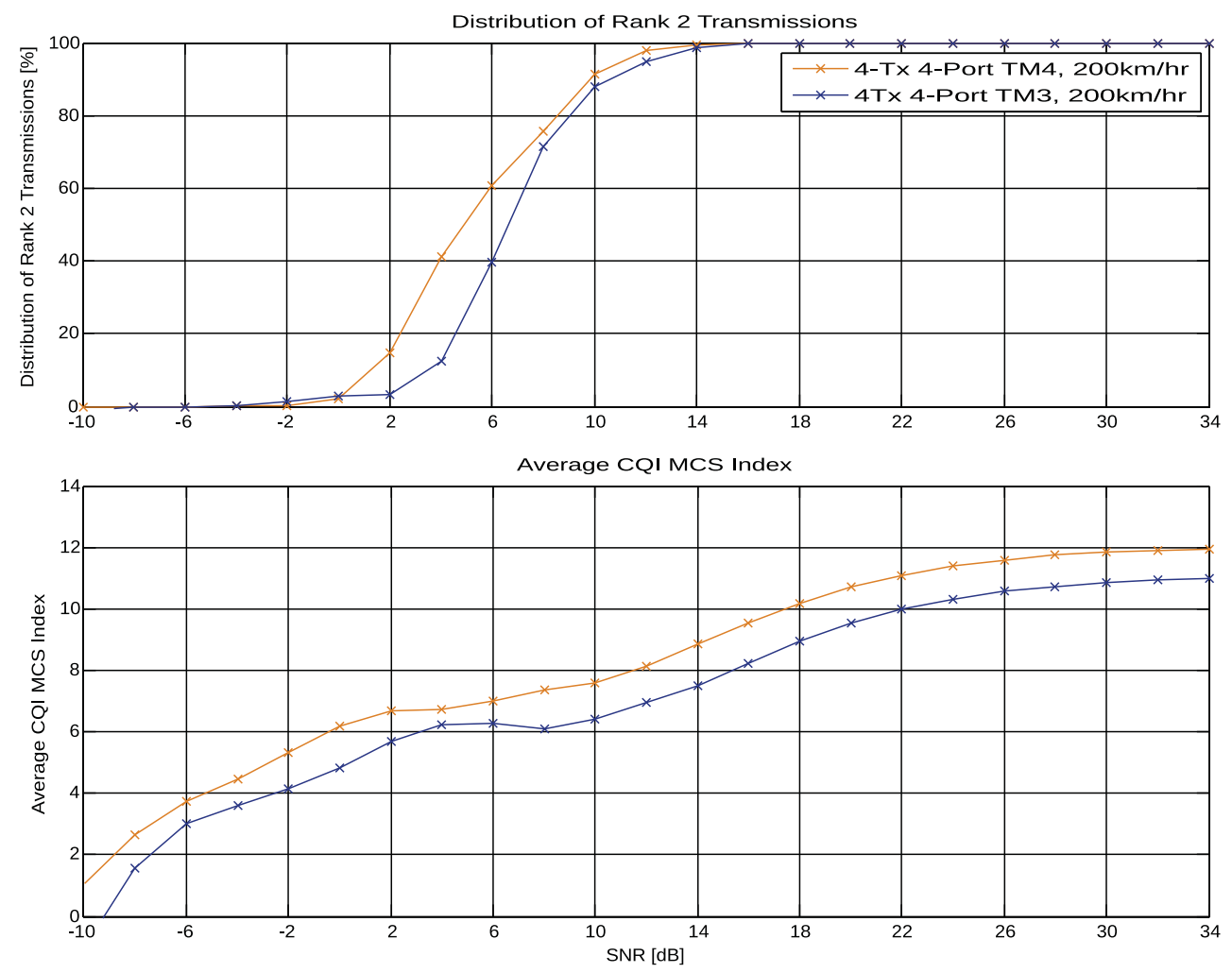

(b) Rank Distribution and Average CQI MCS Index at $200 \mathrm{~km} / \mathrm{hr}$

Figure 4.35: 4-Tx 4-Port High Speed Train Simulation Results at $200 \mathrm{~km} / \mathrm{hr}$ 


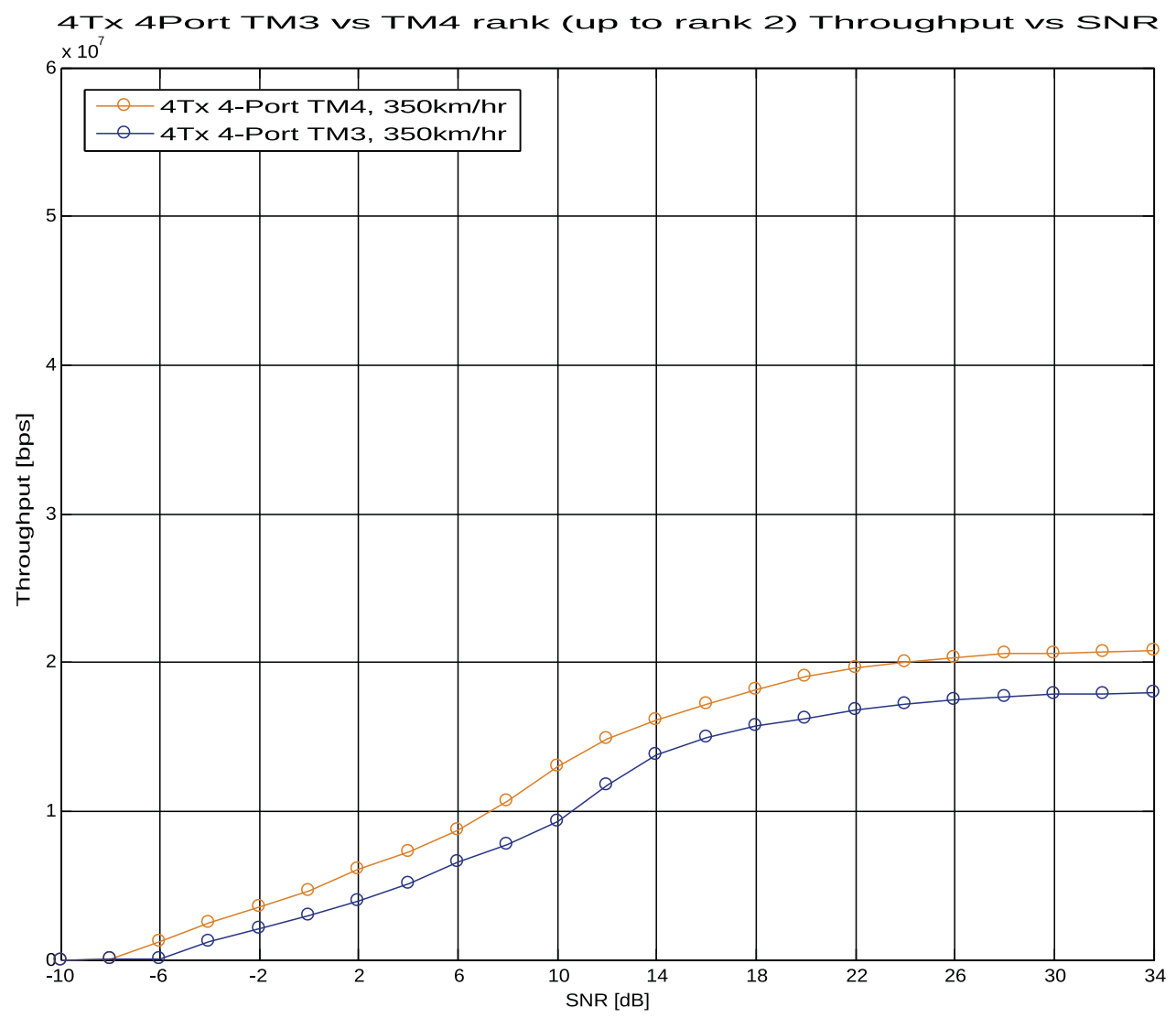

(a) Throughput vs SNR at $350 \mathrm{~km} / \mathrm{hr}$
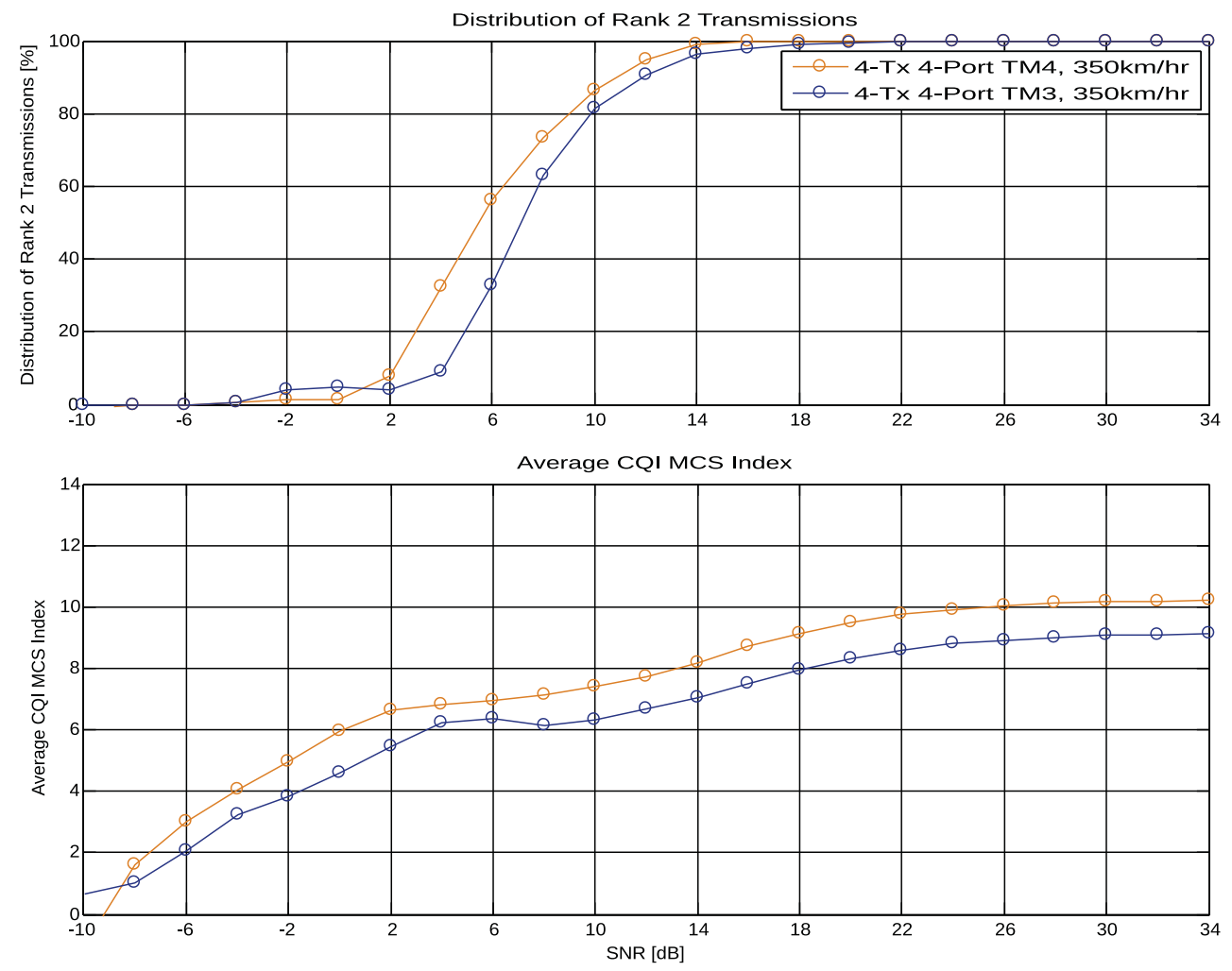

(b) Rank Distribution and Average CQI MCS Index at $350 \mathrm{~km} / \mathrm{hr}$

Figure 4.36: 4-Tx 4-Port High Speed Train Simulation Results at $350 \mathrm{~km} / \mathrm{hr}$ 
UE velocity increase in table 4.19. A system that provided a peak throughput greater than $50 \mathrm{Mbps}$ at $2.1 \mathrm{~km} / \mathrm{hr}$ only provides a peak throughput of $18.0 \mathrm{Mbps}$ (TM3) or $20.8 \mathrm{Mbps}$ (TM4) at $350 \mathrm{~km} / \mathrm{hr}$; a significant reduction in system performance. As the UE velocity increases, the receiver's task becomes increasingly difficult since the channel estimation and analysis become noisy causing both increased decoding failures and difficulties selecting the new optimal channel state information due to errors introduced by the rapid changing channel.

Table 4.20 is a summary of the average CQI index reported to the eNodeB via the uplink. In this case, no outer loop link adaptation was used to see how the reported MCS would affect the downlink throughput. The CQI index is mostly unaffected by the increase in velocity at $10 \mathrm{~dB}$ SNR for both transmission modes. This is not the case at $34 \mathrm{~dB}$ SNR where the average CQI index drops by 2.7 and 2.5 for TM3 and TM4. Therefore, at least a portion of the large drop in throughput seen at $34 \mathrm{~dB}$ SNR in table 4.19 can be attributed to the MCS based on the UE's CQI selection.

Table 4.20: Average CQI Index at Increasing UE Velocity

\begin{tabular}{c|lc|ll}
\hline UE & \multicolumn{2}{|c|}{ Avg CQI Index at 10 dB SNR } & \multicolumn{2}{|c}{ Avg CQI Index at 34 dB SNR } \\
Velocity & TM3 & TM4 & TM3 & TM4 \\
\hline \hline $2.1 \mathrm{~km} / \mathrm{hr}$ & 6.3 & 7.8 & 11.8 & 12.7 \\
$124.6 \mathrm{~km} / \mathrm{hr}$ & 6.4 & 7.7 & 11.6 & 12.5 \\
$200 \mathrm{~km} / \mathrm{hr}$ & 6.4 & 7.6 & 11.0 & 11.9 \\
$350 \mathrm{~km} / \mathrm{hr}$ & 6.3 & 7.4 & 9.1 & 10.2 \\
\hline
\end{tabular}

Table 4.21 is a summary of the percentage of rank-2 transmissions that occurred during the high speed train simulations. The larger drop in throughput at high SNR does not correlate well with the small reduction in rank-2 transmission observed at $34 \mathrm{~dB}$ SNR. It is possible that the eNodeB continued to transmit 2 codewords despite decoding failures at the UE. In the low SNR case the number of rank-2 transmission drops significantly $(76.3 \%$ and $70.3 \%$ down to $21.1 \%$ and $44.3 \%$ for TM3 and TM4 respectively). The throughput and CQI index may have been less affected by UE velocity due to the more robust rank-1 transmissions being transmit from the eNodeB at $10 \mathrm{~dB}$ SNR. However, in order to properly verify the increased rank and throughput degradation relation, more results such as BLER 
Table 4.21: Distribution of Rank 2 Transmissions at Increasing UE Velocity

\begin{tabular}{c|cc|cc}
\hline & \multicolumn{2}{|c|}{ Percentage of Rank 2 } & \multicolumn{2}{c}{ Percentage of Rank 2 } \\
UE & Transmissions at 5 dB SNR & Transmissions at 10 dB SNR \\
Velocity & TM3 & TM4 & TM3 & TM4 \\
\hline \hline $2.1 \mathrm{~km} / \mathrm{hr}$ & $76.3 \%$ & $70.3 \%$ & $96.8 \%$ & $94.1 \%$ \\
$124.6 \mathrm{~km} / \mathrm{hr}$ & $30.8 \%$ & $54.4 \%$ & $89.1 \%$ & $90.5 \%$ \\
$200 \mathrm{~km} / \mathrm{hr}$ & $26.0 \%$ & $51.0 \%$ & $87.8 \%$ & $91.3 \%$ \\
$350 \mathrm{~km} / \mathrm{hr}$ & $21.1 \%$ & $44.3 \%$ & $81.5 \%$ & $86.5 \%$ \\
\hline
\end{tabular}

or BER are needed.

In 2012, Ericsson [44] conducted tests involving a jet flying above the tree-line toward a base station at $600 \mathrm{~km} / \mathrm{hr}$ and $500 \mathrm{~km} / \mathrm{hr}$ to test an internet connection and a seamless hand-over while streaming video and both were successful. In addition to this, a maximum downlink speed of a system capable of over $100 \mathrm{Mbps}$ was able to maintain $19 \mathrm{Mbps}$ at 700 $\mathrm{km} / \mathrm{hr}$. The field test conducted by Ericsson and presented in [44] agrees with the results in this thesis that speeds beyond high speed train velocities are possible due to LTE's sound and forward looking design. 


\section{Chapter 5}

\section{Conclusion and Future Work}

LTE is a new wireless standard that has recently been deployed in commercial networks. The demand for more bandwidth led the 3GPP to develop a flexible OFDM based technology that can operate in wide bandwidths and aggregate spectrum if necessary to provide high bit rates and good quality of service to the consumer. Many studies have looked at the performance of individual algorithms and sub-blocks of wireless systems. This thesis used a complete LTE link level simulator based on the advanced extended spatial channel model and configured with practical parameters. The simulations and discussions presented looked at how practical system impairments and system configurations affected the downlink throughput.

\subsection{Conclusion}

In this thesis, the reader was introduced to the wireless channel and the effects that the channel has on the transmitted OFDM signals. Basic propagation and channel modeling was introduced and then followed by a more detailed account of the models and work that lead to the extended spatial channel model. The concept of a drop, sub-paths, path loss and cluster modeling was explained in section 2.3.7 to give the reader a foundation to understand complex spatial channel modeling and the reason for the use of transmission modes in LTE to overcome the channel effects.

In chapter 2, the inner workings of the LTE physical layer was described, including the OFDM transmissions, the downlink radio frame, channel estimation, link adaptation and the various transmission modes used to obtain the simulation results found in chapter 4

In chapter 4, the topics discussed in the objectives section of this thesis were presented and discussed in detail in order to provide answers to the questions posed in section 1.6. The 
motivation for the study of each topic was presented and a simulation scenario was given. Each LTE link level simulation was briefly described and the detailed system parameters were given for each simulation and then followed by a discussion of the presented figures.

The three topics covered by the studies are:

1. Antenna configuration performance using the following 3 antenna options:

- Number of antennas

- Antenna polarization configuration

- Antenna correlation

2. Phase noise effects on the transmit OFDM signals.

3. The effect of UE velocity and practical channel state information (CSI) on the downlink.

The throughput results based on the SCME simulations were performed at a particular point of interest in space which is advantageous when the effects of different transmission settings needs to be observed. While time-averaging was used over a large number of sub-frames (15,000 sub-frames) the results predicted by the SCME model are specific to the chosen location in space (ie. the network layout) and channel realization because they result in a particular drop. This is useful for the comparisons made in chapter 4 but will vary if a new location and channel seed was selected.

Section 4.1 discussed the topic of multiple antennas and the performance of various antenna array configurations. The simulations provided the reader with a comparison of the fall-back mode (TM2) and TM4 rank-1 and determined that the fall-back mode may not be required based on the SCME simulations and the low velocity EPA simulations.

In section 4.1.6, a number of antenna configurations are simulated and closed-loop spatial multiplexing is shown to provide the highest overall throughput when using the 4-Tx 4 -port $0.7 \lambda$-spaced cross-polarized antennas at the eNodeB compared to the other antenna configurations evaluated using the SCME and EPA models. As the UE velocity increased, 4-Tx 4-port TM4 was still the best mode (with $0.7 \lambda$-spaced cross-polarized antennas) but with other sub-optimal but still useful antenna configurations are used ${ }^{1}, 2-T x$ 2-port TM3 and 2-Tx 2-port TM4 offer similar throughput with a higher peak throughput than 4-Tx 4port TM4. If two antenna ports are used with 2 or $4 \mathrm{BS}$ antennas, it is important to note that 2-port TM3 begins to outperform 2-port TM4 as UE velocity increases. The $0.7 \lambda$-spaced

\footnotetext{
1 "Sub-optimal but useful" antenna configurations refer to the $10 \lambda$-spaced co-polarized and the $10 \lambda$ spaced cross-polarized base station antennas.
} 
co-polarized base station array was also shown not to provide useful results since rank-2 transmission were not successfully transmit at medium and high SNR.

Section 4.2 discussed the effects of phase noise on the performance of the LTE downlink. Reference simulations were conducted with the EPA channel model using TM2 and TM4 rank-1 with three levels of generated phase noise. The amount of generated phase noise added to the simulations helped understand the effects of the lesser real phase noise added in section 4.2.3. If the gathered phase noise had been more significant, the throughput of the simulations using the gathered phase noise may have been reduced. However, in this case, the measured system phase noise did not cause degradation due to the low noise level and slowly changing noise processes.

In section 4.3, throughput results versus UE velocity and link adaptation parameters were presented. The simulations were conducted with 2 and 4 transmitters at the base station and 2 and 4 ports along with $0.7 \lambda$-spaced cross-polarized antennas. The UE velocity simulations demonstrated how the practical parameters of the link adaptation control loop affects the throughput of the LTE downlink. When practical link adaptation parameters are used, the drop in throughput occurs well under $30 \mathrm{~km} / \mathrm{hr}$. As mentioned, the processing delay is a key factor causing the rapid degradation of the throughput since the channel state information is no longer applicable and link adaptation no longer tracks the rapid channel fluctuations. Any improvement to the user UE and base station processing delay does not increase overhead on the uplink but helps increase the efficiency of the LTE downlink when the UE is mobile. In these simulations 4-Tx 4-port TM4 is shown to provide the highest throughput overall. On the other hand, if 2 antenna ports are used, 2-port TM4 is better when the UE is quasi-stationary but 2-port TM3 begins to outperform 2-port TM4 as UE becomes mobile.

Finally, the high speed train results showed that the LTE system is well designed to handle very high UE velocities and Doppler shifts, but that the throughput does decline considerably from $124.6 \mathrm{~km} / \mathrm{hr}$, to $200 \mathrm{~km} / \mathrm{hr}$ and again at $350 \mathrm{~km} / \mathrm{hr}$.

\subsection{Future Work}

The simulation results presented in this thesis are based on the 3GPP EPA conformance and testing channel model and the extended spatial channel model (SCME). These models attempt to reproduce the channel effects that have been observed by researchers by taking measurements in typical environments. Future work could include verifying the results by 
setting up a similar scenario outdoors and taking measurements at a similar distance from the base station as the distance (550 meters) used in this thesis. While this would be a very interesting experiment it may be difficult to reproduce the exact results presented. Instead, a more useful measurement would be to perform drive testing under similar circumstances to obtain average data and determine if the results in this thesis match the trends seen in the drive tests.

In each simulation the location within the cell was selected to give representative results and one location per simulation was reasonable in order to compare the throughput, especially since a single variable was being changed to compare throughput curves. For each simulated topic in this thesis an increase in the number of drops simulated would help give a better picture of the performance of the downlink across the cell. Future work could include validating the results obtained under different channel scenarios and at different locations in the cell (ie. multiple drops) or near the cell edge, for instance. A larger set of results would provide added confidence in the data presented in this thesis, if the results agreed.

The scenario used to simulate the high speed train results in section 4.3.3 employs two closely spaced cross-polarized antennas at the UE. In the future, implementations of an LTE railway radio access network is likely to include a repeater on the roof of the train cars carrying passengers. In order to be more representative of the potential results achievable with a repeater mounted on the roof of a train, a more complex simulation might be required to model the system level issues present. However, an improvement in the accuracy of the simulations at the physical layer could be achieved by repeating the simulations in this section with widely spaced UE antennas to model the possible repeater antenna setup that could be used on a train car roof. With a repeater mounted on the roof of a train car, the antennas could be spaced as far apart as the length of the roof of the train car. The train cars are typically long enough to achieve spacings that are tens of multiples of the wavelength of the propagating LTE radio waves. Simulations with widely spaced "repeater" UE antennas may improve spatial diversity, compared to co-located cross-polarized antennas, in a LTE railway radio access network and provide increased throughput.

Another improvement in the high speed train study could be to incorporate more appropriate path loss models and add the Doppler shift profile from [4] to the extended spatial channel model simulations similar to the work done in [41] which incorporated a Doppler profile and new path loss models into the WINNER WP1 D2a channel model. Anytime more representative models can be used, the accuracy and confidence in the results should 
improve.

Finally, future work could also include investigating any LTE weaknesses brought to light in the topics presented. For instance, an attempt could be made to address the throughput decline that occurs as UE velocity increases. New algorithms that adapt reference signal patterns or new low complexity calculations to reduce channel state indicator processing delays would be beneficial to improving the performance of LTE at very high UE velocities. As another example, the system performance could be improved by making better decisions about the transmission modes. Based on the results seen here it should be possible to take advantage of the strengths of each evaluated transmission mode by switching between the modes as the channel changes (eg. Doppler increase). 


\section{Appendix A}

\section{A.1 4-bit Channel Quality Indicator Table}

The 4-bit channel quality indicator table A.1 is used to report the preferred AMC scheme to the eNodeB. The 4-bit CQI is used to keep the feedback overhead low on the uplink channels PUSCH and PUCCH.

\section{A.2 Modulation and Transport Block Size Index Table for the PDSCH}

The eNodeB transmits the PDSCH using a modulation scheme selected from table A.2 based on the CQI feedback from the UE. The eNodeB informs the UE of the transport block size index $I_{T B S}$ used for PDSCH transmissions by transmitting the DCI on the PDCCH. The UE reads the 5-bit $I_{M C S}$ value from the DCI and the resource allocation field to determine the number of resource block group (a set of consecutive physical resource blocks)s (RBGs) allocated to the UE, if applicable. $N_{P R B}$ and table A.2.

\section{A.3 Resource Block Group Size versus Downlink Band- width (Type 0 Resource Allocation)}

The RBG size in LTE is used to define the size of a group of consecutive PRBs in the LTE system bandwidth in order to identify groups and reduce the amount of feedback on the uplink and downlink control channels. In a resource allocation type 0 , for example, a UE is made aware of the downlink resources that have been allocated to it by reading a bitmap 
Table A.1: 4-bit CQI Table

\begin{tabular}{cccc}
\hline CQI Index & Modulation & Code Rate & Efficiency [bits/s/Hz] \\
\hline \hline 0 & & - No transmission - \\
1 & QPSK & 0.0762 & 0.1523 \\
2 & QPSK & 0.1172 & 0.2344 \\
3 & QPSK & 0.1885 & 0.3770 \\
4 & QPSK & 0.3008 & 0.6016 \\
5 & QPSK & 0.4385 & 0.8770 \\
6 & QPSK & 0.5879 & 1.1758 \\
7 & 16QAM & 0.3691 & 1.4766 \\
8 & 16QAM & 0.4785 & 1.9141 \\
9 & 16QAM & 0.6016 & 2.4063 \\
10 & 64QAM & 0.4551 & 2.7305 \\
11 & 64QAM & 0.5537 & 3.3223 \\
12 & 64QAM & 0.6504 & 3.9023 \\
13 & 64QAM & 0.7539 & 4.5234 \\
14 & 64QAM & 0.8525 & 5.1152 \\
15 & 64QAM & 0.9258 & 5.5547 \\
\hline & & & \\
\hline
\end{tabular}


Table A.2: Modulation and Transport Block Size (TBS) Index Table for the Physical Down-link Shared Channel (PDSCH)

\begin{tabular}{|c|c|c|}
\hline $\begin{array}{l}\text { MCS Index } \\
\qquad I_{M C S}\end{array}$ & $\begin{array}{c}\text { Modulation Order } \\
Q_{m}[\text { Number of bits }]\end{array}$ & $\begin{array}{l}\text { Transport block size (TBS) Index } \\
\qquad I_{T B S}\end{array}$ \\
\hline 0 & 2 & 0 \\
\hline 1 & 2 & 1 \\
\hline 2 & 2 & 2 \\
\hline 3 & 2 & 3 \\
\hline 4 & 2 & 4 \\
\hline 5 & 2 & 5 \\
\hline 6 & 2 & 6 \\
\hline 7 & 2 & 7 \\
\hline 8 & 2 & 8 \\
\hline 9 & 2 & 9 \\
\hline 10 & 4 & 9 \\
\hline 11 & 4 & 10 \\
\hline 12 & 4 & 11 \\
\hline 13 & 4 & 12 \\
\hline 14 & 4 & 13 \\
\hline 15 & 4 & 14 \\
\hline 16 & 4 & 15 \\
\hline 17 & 6 & 15 \\
\hline 18 & 6 & 16 \\
\hline 19 & 6 & 17 \\
\hline 20 & 6 & 18 \\
\hline 21 & 6 & 19 \\
\hline 22 & 6 & 20 \\
\hline 23 & 6 & 21 \\
\hline 24 & 6 & 22 \\
\hline 25 & 6 & 23 \\
\hline 26 & 6 & 24 \\
\hline 27 & 6 & 25 \\
\hline 28 & 6 & 26 \\
\hline $29,30,31$ & & - reserved - \\
\hline
\end{tabular}


of bits sent on the PDCCH. The bits in the bitmap are associated with specific RBGs that represent groups of consecutive PRBs. The RBG size $P$ iis dependent on the bandwidth (measured in PRBs) as shown in table A.3.

Table A.3: Resource Block Group Size vs. Downlink Bandwidth (Type 0 Resource Allocation)

\begin{tabular}{cc}
\hline System Bandwidth & Resource Block Group Size $(P)$ \\
\hline \hline$\leq 10$ & 1 \\
$11-26$ & 2 \\
$27-63$ & 3 \\
$64-110$ & 4 \\
\hline
\end{tabular}




\section{Appendix B}

\section{B.1 Jakes' Model}

Jakes' model from [9] is a widely known model for mobile radio simulation.

$$
\begin{gathered}
g_{(}(t)=g_{c}(t)+j g_{s}(t) \\
g_{c}(t)=\frac{2}{\sqrt{N}} \sum_{m=1}^{M+1} a_{n} \cos \left(\omega_{n} t\right) \\
g_{s}(t)=\frac{2}{\sqrt{N}} \sum_{m=1}^{M+1} b_{n} \cos \left(\omega_{n} t\right) \\
N=4 M+2
\end{gathered}
$$

Where the equations for $a_{n}, b_{n}, \beta_{n}$ and $\omega_{n}$ are given by:

$$
\begin{gathered}
\beta_{n}= \begin{cases}\pi n / M, & n=1,2, \ldots, M \\
\pi / 4, & n=M+1\end{cases} \\
\omega_{n}= \begin{cases}\omega_{d} \cos (2 \pi n / N) & n=1,2, \ldots, M \\
\omega_{d}, & n=M+1\end{cases} \\
a_{n}= \begin{cases}2 \cos \beta_{n}, & n=1,2, \ldots, M \\
\sqrt{2} \cos \beta_{M+1}, & n=M+1\end{cases}
\end{gathered}
$$


APPENDIX B.

$$
b_{n}= \begin{cases}2 \sin \beta_{n}, & n=1,2, \ldots, M \\ \sqrt{2} \sin \beta_{M+1}, & n=M+1\end{cases}
$$




\section{Appendix C}

\section{C.1 Peak Theoretical Throughput for LTE Downlink Transmissions}

\section{C.1.1 Peak Theoretical Throughput for 8 PRB UE Allocation using Single Codeword Transmissions}

The peak theoretical throughput for a LTE FDD system using 4 transmitters and 4 ports is calculated below. The following system and transmission settings are used:

- Cyclic prefix length: normal

- OFDM symbols per sub-frame: $N_{S y m b}^{S F}=14$ OFDM Symbols

- Sub-carriers per PRB: $N_{s c}^{R B}=12$ sub-carriers

- Number of OFDM symbols used for PDCCH: $N_{P D C C H}^{S F}$ Symb $=3$ OFDM symbols

- Number of overlapping PDCCH and RS resource elements: $N_{R S}^{P D C C H}=8$

- Number of PRB allocated to the UE: $N_{P R B}^{U E}=8$

- Number of codewords: 1

- Modulation order and bits/symbol: $M=64$ for 64QAM and $Q_{m}=6$

- LTE maximum turbo coding rate: $R_{\max }=0.93$

The theoretical throughput is calculated by finding the number of available data REs per sub-frame and multiplying by the modulation order, maximum code rate and number of PRB allocated to the UE. The calculation assumes that the system is not heavily loaded and the eNodeB is capable of scheduling the UE outside the central 6 PRBs where the synchronization signals would add additional overhead. 
Throughput for 8 PRBs [Mbps] $=\frac{N_{P R B}^{U E} \cdot N_{\text {Data REs }}^{S F} \cdot Q_{m} \cdot R_{\text {max }} \cdot 1000 \frac{\text { Sub-frames }}{\text { Second }}}{10^{6}}$

$$
\begin{aligned}
N_{R E}^{S F} & =N_{s c}^{R B} \cdot N_{S y m b}^{S F}=168 \mathrm{REs} \\
N_{R S}^{S F} & =(8 \mathrm{CRS} \cdot 2 \text { ports })+(4 \mathrm{CRS} \cdot 2 \text { ports })=24 \mathrm{REs} \\
N_{P D C C H R E}^{S F} & =\left(N_{P D C C H S y m b}^{S F} \cdot N_{s c}^{R B}\right)-N_{R S}^{P D C C H}=28 \mathrm{REs} \\
N_{\text {Data REs }}^{S F} & =N_{R E}^{S F}-\left(N_{P D C C H R E}^{S F}+N_{R S}^{S F}\right)=116 \text { data REs }
\end{aligned}
$$

Throughput for 8 PRBs $[\mathrm{Mbps}]=\frac{8 \mathrm{PRBs} \cdot 116 \mathrm{REs} \cdot 6 \frac{\mathrm{bits}}{\mathrm{RE}} \cdot 0.93 \cdot 1000 \frac{\text { Sub-frames }}{\text { Second }}}{10^{6}}$

$$
=5.178 \mathrm{Mbps}
$$

The reader should note that the peak theoretical throughput calculation shown in equation C. 1 does not account for the discrete transport block sizes that are defined in the LTE standard. The peak theoretical throughput in appendix C.1.2 is more realistic since the calculation accounts for the discrete transport block sizes and maximum code rate permissible $\left(R_{\max }=0.93\right)$ in LTE.

\section{C.1.2 Table of Peak Theoretical Throughput Values for Various Con- figurations used in this Thesis}

Table C.1 is a list of theoretical peak throughput values for an LTE FDD system using various transmission settings.

The theoretical peak throughput is calculated by counting the number of data REs available to the PDSCH after the reference signals and control channels have been allocated. The reference signals are allocated as described in section 2.4.4 and the control channel fill up the remaining REs of the first three OFDM symbols of the normal (12 x 14) sub-frame. The highest modulation order and largest transport block size that meet the LTE code rate limit of 0.93 is selected. The number of PDSCH REs was then multiplied by the modulation and code rate combination found by exhaustive search in the previous step to calculate the peak physical layer throughput based on the discrete transport block size and MCS available.

The peak throughput results where the UE is allocated 8 PRB assume that the system 
Table C.1: Peak Theoretical Throughput Values for Various Transmission Settings using the LTE Transport Block Size Table

\begin{tabular}{cccc}
\hline $\begin{array}{c}\text { Resource } \\
\text { Allocation }\left(N_{P R B}^{U E}\right)\end{array}$ & Codewords & $\begin{array}{c}\text { Antenna } \\
\text { Ports }(P)\end{array}$ & $\begin{array}{c}\text { Max Throughput (using } \\
\text { transport block size table) }\end{array}$ \\
\hline \hline 8 & 1 & 2 & $5.16 \mathrm{Mbps}$ \\
8 & 2 & 2 & $10.32 \mathrm{Mbps}$ \\
8 & 1 & 4 & $4.968 \mathrm{Mbps}$ \\
8 & 2 & 4 & $9.936 \mathrm{Mbps}$ \\
50 & 1 & 2 & $31.7 \mathrm{Mbps}$ \\
50 & 2 & 2 & $63.41 \mathrm{Mbps}$ \\
50 & 1 & 4 & $31.7 \mathrm{Mbps}$ \\
50 & 2 & 4 & $63.41 \mathrm{Mbps}$ \\
\hline
\end{tabular}

is not heavily loaded and the eNodeB is capable of scheduling the UE outside the central 6 PRBs where the synchronization signals would add additional overhead. When all the PRBs are allocated to the UE in the $10 \mathrm{MHz}$ system a small difference may occur since it is assumed here that no system information is transmit in the 6 central PRB. 


\section{Appendix D}

\section{D.1 Correlation Matrices for 3GPP Conformance Testing Channel Models}

BS and UE correlation matrices for 3GPP conformance and testing channel models [6]:

$$
\begin{aligned}
& \mathbf{R}_{\mathbf{B S}}=\left[\begin{array}{ll}
1 & \alpha \\
\alpha^{*} & 1
\end{array}\right] \\
& \mathbf{R}_{\mathbf{U E}}=\left[\begin{array}{ll}
1 & \beta \\
\beta^{*} & 1
\end{array}\right]
\end{aligned}
$$

The channel spatial correlation matrix $\mathbf{R}_{\text {spat }}$ for the 3 GPP conformance and testing channel models [6] is calculated using:

$$
\mathbf{R}_{\text {spat }}=\mathbf{R}_{\mathbf{B S}} \otimes \mathbf{R}_{\mathbf{U E}}=\left[\begin{array}{cc}
1 & \beta \\
\beta^{*} & 1
\end{array}\right] \otimes\left[\begin{array}{cc}
1 & \alpha \\
\alpha^{*} & 1
\end{array}\right]
$$

The available correlation choices for the extended-ITU channel spatial correlation matrices: 
Table D.1: Extended-ITU Spatial Correlation Parameters $\alpha$ and $\beta$

\begin{tabular}{cccccc}
\hline \multicolumn{2}{c}{ Low Correlation } & \multicolumn{2}{c}{ Medium Correlation } & \multicolumn{2}{c}{ High Correlation } \\
$\alpha$ & $\beta$ & $\alpha$ & $\beta$ & $\alpha$ & $\beta$ \\
\hline \hline 0 & 0 & 0.3 & 0.9 & 0.9 & 0.9 \\
\hline
\end{tabular}

The resulting low correlation matrix for $2 \times 2 \mathrm{MIMO}[6]$ is:

$$
R_{\text {low }}=\left[\begin{array}{llll}
1 & 0 & 0 & 0 \\
0 & 1 & 0 & 0 \\
0 & 0 & 1 & 0 \\
0 & 0 & 0 & 1
\end{array}\right]
$$

The resulting medium correlation matrix for $2 \times 2 \mathrm{MIMO}[6]$ is:

$$
R_{\text {medium }}=\left[\begin{array}{cccc}
1 & 0.9 & 0.3 & 0.27 \\
0.9 & 1 & 0.27 & 0.3 \\
0.3 & 1 & 0.27 & 0.3 \\
0.27 & 0.3 & 0.9 & 1
\end{array}\right]
$$

The resulting high correlation matrix for $2 \times 2$ MIMO [6] is:

$$
R_{\text {high }}=\left[\begin{array}{cccc}
1 & 0.9 & 0.9 & 0.81 \\
0.9 & 1 & 0.81 & 0.9 \\
0.9 & 0.81 & 1 & 0.9 \\
0.81 & 0.9 & 0.9 & 1
\end{array}\right]
$$




\section{List of References}

[1] 3GPP, "Evolved universal terrestrial radio access (E-UTRA); physical channels and modulation," TS 36.211 Release 11, 3rd Generation Partnership Project (3GPP), Sept. 2012.

[2] C. Xiao, Y. R. Zheng, and N. C. Beaulieu, "Novel sum-of-sinusoids simulation models for rayleigh and rician fading channels," Wireless Communications, IEEE Transactions on, vol. 5, pp. 3667-3679, Dec. 2006.

[3] 3rd Generation Partnership Project (3GPP), "Technical specification group radio access network; spatial channel model for multiple input multiple output (MIMO) simulations," TR 25.996 Release 11, 3rd Generation Partnership Project (3GPP), Sept. 2012.

[4] 3GPP, "Evolved universal terrestrial radio access (E-UTRA); base station (BS) radio transmission and reception," TS 36.104 Release 11, 3rd Generation Partnership Project (3GPP), Mar. 2013.

[5] R. H. Clarke, "A statistical theory of mobile radio reception," Bell Labs System Technical Journal, vol. 47, pp. 957-1000, July-August 1968.

[6] 3GPP, "Evolved Universal Terrestrial Radio Access (E-UTRA); User Equipment (UE) radio transmission and reception," TS 36.101 Release 11, 3rd Generation Partnership Project (3GPP), Sept. 2012.

[7] T. Rappaport, Wireless communications: principles and practice. Prentice Hall, second ed., 2002.

[8] J. Proakis and M. Salehi, Digital Communications. McGraw-Hill, fifth ed., 2007.

[9] W. Jakes, Microwave mobile communications. New York: Wiley, 1974.

[10] M. F. Pop and N. C. Beaulieu, "Limitations of sum-of-sinusoids fading channel simulators," Communications, IEEE Transactions on, vol. 49, pp. 699-708, Apr. 2001.

[11] E. Gilbert, "Energy reception for mobile radio," Bell Systems Technical Journal, vol. 44, pp. 1779-1803, Oct. 1965.

[12] W. Young Jr., "Comparison of mobile radio transmission at 150, 450, 900, and 3700 Mc," Bell System Technical Journal, vol. 31, pp. 1068-1085, Nov. 1952.

[13] W. Jakes and D. Reudink, "Comparison of mobile radio transmission at UHF and X band," Vehicular Technology, IEEE Transactions on, vol. 16, pp. 10-14, Oct. 1967. 
[14] C. Xiao, "Rayleigh channel fading simulator: Problems and solutions," BCWS slides, Dept. of Electrical $\mathcal{E}$ Computer Engineering, June 2002.

[15] Y. S. Cho, J. Kim, W. Y. Yang, and C. G. Kang, MIMO-OFDM Wireless Communications with Matlab. John Wiley \& Sons, 2010.

[16] 3rd Generation Partnership Project (3GPP), “Technical specification group radio access network; spatial channel model for multiple input multiple output (MIMO) simulations," TR 25.996 Release 6.1.0, 3rd Generation Partnership Project (3GPP), Oct. 2003.

[17] D. Baum, J. Hansen, and J. Salo, "'”an interim channel model for beyond-3G systems: extending the 3GPP spatial channel model (SCM)"," in Vehicular Technology Conference, 2005. VTC 2005-Spring. 2005 IEEE 61st, vol. 5, pp. 3132-3136, IEEE, 2005.

[18] 3GPP, "Evolved universal terrestrial radio access (E-UTRA); multiplexing and channel coding," TS 36.212 Release 11, 3rd Generation Partnership Project (3GPP), Sept. 2012.

[19] T. Moon, Error Correction Coding. New Jersey: Wiley-Interscience, June 2005.

[20] J. W. Choi and Y. H. Lee, "Design of the optimum pilot pattern for channel estimation in OFDM systems," in Global Telecommunications Conference, 2004. GLOBECOM '04. IEEE, vol. 6, pp. 3661-3665, 2004.

[21] P. Hoeher, S. Kaiser, and P. Robertson, "Two-dimensional pilot-symbol-aided channel estimation by Wiener filtering," in Acoustics, Speech, and Signal Processing, 1997. ICASSP-97., 1997 IEEE International Conference on, vol. 3, pp. 1845-1848 vol.3, 1997.

[22] 3rd Generation Partnership Project (3GPP), "Evolved universal terrestrial radio access (E-UTRA); multiplexing and channel coding," TS 36.213 Release 11, 3rd Generation Partnership Project (3GPP), Sept. 2012.

[23] S. M. Alamouti, "A simple transmit diversity technique for wireless communications," Selected Areas in Communications, IEEE Journal on, vol. 16, pp. 1451-1458, Oct. 1998.

[24] G. Ganesan and P. Stoica, "Space-time block codes: a maximum SNR approach," Information Theory, IEEE Transactions on, vol. 47, pp. 1650-1656, May 2001.

[25] F. Khan, LTE for 4G Mobile Broadband: Air Interface Technologies and Performance. New York: Cambridge University Press, 2009.

[26] M. Bossert, A. Huebner, F. Schuehlein, H. Haas, and E. Costa, "On cyclic delay diversity in OFDM based transmission schemes," in OFDM workshop, vol. 2, 2002.

[27] S. Schwarz, M. Wrulich, and M. Rupp, "Mutual information based calculation of the precoding matrix indicator for 3gpp umts/lte," in Smart Antennas (WSA), 2010 International ITG Workshop on, pp. 52-58, 2010. 
[28] S. Schwarz, C. Mehlfuhrer, and M. Rupp, "Calculation of the spatial preprocessing and link adaption feedback for 3gpp umts/lte," in Wireless Advanced (WiAD), 2010 6th Conference on, pp. 1-6, 2010.

[29] S. Schwarz and M. Rupp, "Throughput maximizing feedback for MIMO OFDM based wireless communication systems," in Signal Processing Advances in Wireless Communications (SPAWC), 2011 IEEE 12th International Workshop on, pp. 316-320, June 2011.

[30] G. Caire, G. Taricco, and E. Biglieri, "Capacity of bit-interleaved channels," Electronics Letters, vol. 32, no. 12, pp. 1060-1061, 1996.

[31] ITU-R Recommendations, "ITU-R Recommendation M.1225: Guidelines for evaluation of radio transmission technologies for IMT-2000," no. M.1225, p. 60, 1997.

[32] A. S. Pags, “A long term evolution link level simulator," Master's thesis, Department of Signal Theory and Communications, Catalonia, Spain, Feb 2009.

[33] C. Mehlführer, M. Wrulich, J. C. Ikuno, D. Bosanska, and M. Rupp, "Simulating the long term evolution physical layer," in Proc. of the 17th European Signal Processing Conference (EUSIPCO 2009), Glasgow, Scotland, vol. 27, p. 124, 2009.

[34] P. Mogensen, W. Na, I. Kovacs, F. Frederiksen, A. Pokhariyal, K. Pedersen, T. Kolding, K. Hugl, and M. Kuusela, "LTE capacity compared to the shannon bound," in Vehicular Technology Conference, 2007. VTC2007-Spring. IEEE 65th, pp. 1234-1238, 2007.

[35] P. Suratia and S. Shah, "Performance analysis of open and closed loop spatial multiplexing in LTE downlink physical layer," in Communication, Networks and Satellite (ComNetSat), 2012 IEEE International Conference on, pp. 60-63, 2012.

[36] A. Simonsson, Y. Qian, and J. Ostergaard, "LTE downlink 2x2 MIMO with realistic CSI: Overview and performance evaluation," in Wireless Communications and Networking Conference (WCNC), 2010 IEEE, pp. 1-6, 2010.

[37] J. Furuskog, K. Werner, M. Riback, and B. Hagerman, "Field trials of LTE with 4x4 MIMO," Ericsson Review, 2010.

[38] S. Sesia, I. Toufik, and M. Baker, LTE, The UMTS Long Term Evolution: From Theory to Practice. John Wiley \& Sons, 2009.

[39] G.-C. Zhu, F. Alajaji, J. Bajcsy, and P. Mitran, "OFDM systems in the presence of phase noise: Consequences and solutions," Communications, IEEE Transactions on, vol. 52, no. 5, pp. 855-855, 2004.

[40] D. Tse and P. Viswanath, Fundamentals of Wireless Communication. Cambridge University Press, May 2005.

[41] K. Guan, Z. Zhong, and B. Ai, "Assessment of LTE-R using high speed railway channel model," in Communications and Mobile Computing (CMC), 2011 Third International Conference on, pp. 461-464, 2011. 
[42] IST, "IST-4-027756 WINNER II D1.1.2 V1.0 WINNER II channel models," TR D1.1.2 V1.2, Information Society Technologies, Feb. 2008.

[43] H. Wei, Z. Zhong, K. Guan, and B. Ai, "Path loss models in viaduct and plain scenarios of the high-speed railway," in Communications and Networking in China (CHINACOM), 2010 5th International ICST Conference on, pp. 1-5, 2010.

[44] Ericsson, "Ericsson tests LTE in extreme conditions." http: //www . ericsson . com/ news/121101-ericsson-tests-lte-in-extreme-conditions_244159017_ c, 2012. [Online; accessed 03-April-2013]. 